\title{
ANÁLISIS EXPERIMENTAL DEL FRESADO DE MATERIALES COMPUESTOS REFORZADOS CON FIBRA DE BASALTO
}

\section{TESIS DOCTORAL}

Presentada por:

María Dolores Navarro Mas

Dirigida por:

Ana Isabel Sánchez Galdón

María Desamparados Meseguer Calás

Valencia, Septiembre de 2021 

A mi familia 

Quiero agradecer a todos los que me habéis acompañado estos años durante la realización de mi tesis doctoral.

En especial a mis directoras Amparo y Ana, y a mis compañeros del Área de Ingeniería de los Procesos de Fabricación. 



\section{RESUMEN}

Los materiales compuestos reforzados con fibra son ampliamente utilizados en diversos sectores, como el aeroespacial y el de automoción. Tras su conformado, estos materiales necesitan procesos de mecanizado para eliminar el material sobrante y facilitar la unión entre piezas. Debido a la naturaleza heterogénea y la anisotropía de estos materiales, su comportamiento ante el mecanizado es diferente que los metálicos. Las fibras abrasivas provocan un rápido desgaste de la herramienta y, además, la aparición del delaminado puede hacer necesario el reprocesado o rechazo de las piezas. El delaminado es un defecto que provoca la separación de capas del material compuesto y hace que la pieza presente un aspecto deshilachado en el borde mecanizado porque la herramienta dobla las fibras en vez de cortarlas, además de poder producir un daño superficial en la pieza.

En esta tesis se ha abordado el estudio experimental del fresado de materiales compuestos reforzados con fibra de basalto, en concreto, en la operación de contorneado. En el mecanizado de este material se ha estudiado el desgaste de la herramienta, el acabado superficial y el delaminado, analizando la influencia de los parámetros de corte (velocidad, avance y profundidad de pasada), y de las características del material compuesto (volumen y orientación de fibra). Con respecto al material de las fibras, casi todos los estudios existentes se han centrado en las fibras de carbono y de vidrio, no habiendo estudios con fibras de basalto, aunque este material es de origen natural y una buena alternativa a las fibras de vidrio. Con respecto al proceso de fresado, los estudios existentes en la bibliografía se han centrado más en la operación de ranurado que en la de contorneado.

Una parte fundamental de la tesis se ha centrado en el estudio del delaminado. Para ello se han definido y desarrollado los diferentes aspectos teóricos que caracterizan este defecto. Además, se han cuantificado los tipos de delaminado (Tipos I y II) mediante la definición y comparación de parámetros unidimensionales y de superficie, evaluando su evolución temporal. Los parámetros unidimensionales permiten evaluar la longitud de las fibras que sobresalen o dañan la superficie, mientras que los parámetros de superficie permiten cuantificar la densidad del delaminado.

Como complemento al estudio experimental, se han utilizado herramientas estadísticas (diseño de experimentos, ANOVA y modelos de regresión cuantílica) para estudiar el fresado de este tipo de materiales en relación a la rugosidad, el desgaste de la herramienta y el delaminado. 



\section{ABSTRACT}

Fiber reinforced composites are widely used in diverse fields such as aeronautics and automotive. After their shaping, these materials require machining processes to remove excess material and facilitate joining between parts. Due to the heterogeneous nature and anisotropy of these materials, their behavior when being machined is different from metallic ones. Abrasive fibers trigger fast tool wear and delamination appearance can cause piece reprocessing or discard. Delamination is a defect which induces the separation of composite layers and makes the piece present a frayed appearance on the machined edge because the tool bends the fibers instead of cutting them, in addition to producing surface damage to the piece.

In this thesis, the experimental study of milling of basalt fiber reinforced composite materials has been approached, specifically, in edge trimming. In the machining of this material, tool wear, surface finish and delamination have been studied, analyzing the influence of cutting parameters (cutting speed, feed per tooth and depth of cut) and characteristics of the composite material (fiber volume fraction and fiber orientation). Regarding the fiber material, almost all existing studies have focused on carbon and glass fibers, there are no studies with basalt fibers, although this material is of natural origin and a good alternative to glass fibers. About the milling process, existing studies in the literature have focused more on the grooving operation than on the edge trimming operation.

A fundamental part of the thesis has focused on the study of delamination. To characterize this defect, different theoretical aspects have been defined and developed. Additionally, different types of delamination (types I and II) have been quantified through the definition and comparison of one-dimensional and surface parameters, evaluating their temporal evolution. One-dimensional parameters allow the evaluation of the length of the fibers that protrude or damage the surface, while the surface parameters allow the quantification of the density of delaminate.

As a complement to the experimental study, statistical tools (experiment design, ANOVA and quantile regression models) have been used to study the milling of this type of materials and predict certain roughness, tool wear, and delamination aspects. 



\section{RESUM}

Els materials compostos reforçats amb fibra són àmpliament utilitzats en diversos sectors, com l'aeroespacial i el d'automoció. Després del seu conformat, aquests materials necessiten processos de mecanitzat per a eliminar el material sobrant i facilitar la unió entre peces. A causa de la naturalesa heterogènia i anisotropia d'aquests materials, el seu comportament davant el mecanitzat és diferent que els metàl-lics. Les fibres abrasives provoquen un ràpid desgast de l'eina i, a més, l'aparició de la delaminació pot fer necessari el reprocessament o rebuig de les peces. La delaminació és un defecte que provoca la separació de capes del material compost i fa que la peça presente un aspecte esfilagarsat en la vora mecanitzada perquè l'eina doblega les fibres en lloc de tallar-les, a més de poder produir un mal superficial en la peça.

En aquesta tesi s'ha abordat l'estudi experimental del fresat de materials compostos reforçats amb fibra de basalt, en concret, en l'operació de contornejat. En el mecanitzat d'aquest material s'ha estudiat el desgast de l'eina, l'acabat superficial i la delaminació, analitzant la influència dels paràmetres de tall (velocitat, avanç i profunditat de passada), i de les característiques del material compost (volum i orientació de fibra). Respecte al material de les fibres, quasi tots els estudis existents s'han centrat en les fibres de carboni i de vidre, no havent-hi estudis amb fibres de basalt, encara que aquest material és d'origen natural i una bona alternativa a les fibres de vidre. Respecte al procés de fresat, els estudis existents en la bibliografia s'han centrat més en l'operació de ranurat que en la de contornejat.

Una part fonamental de la tesi s'ha centrat en l'estudi de la delaminació. Per a això s'han definit $\mathrm{i}$ desenvolupat els diferents aspectes teòrics que caracteritzen aquest defecte. A més, s'han quantificat els tipus de delaminació (Tipus I i II) mitjançant la definició i comparació de paràmetres unidimensionals i de superfície, avaluant la seua evolució temporal. Els paràmetres unidimensionals permeten avaluar la longitud de les fibres que sobreïxen o danyen la superfície, mentre que els paràmetres de superfície permeten quantificar la densitat de la delaminació.

Com a complement a l'estudi experimental, s'han utilitzat eines estadístiques (disseny d'experiments, ANOVA i models de regressió quantílica) per estudiar el fresat d'aquesta mena de materials i predir alguns aspectes de la rugositat, el desgast de l'eina i la delaminació. 



\section{ÍNDICE GENERAL}

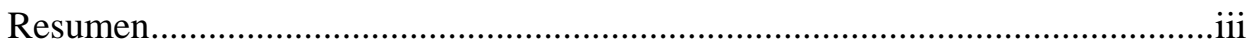

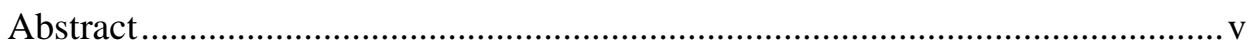

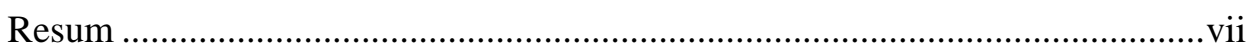

PRESENTACIÓN DE LA TESIS ....................................................................... 1

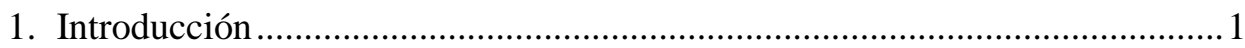

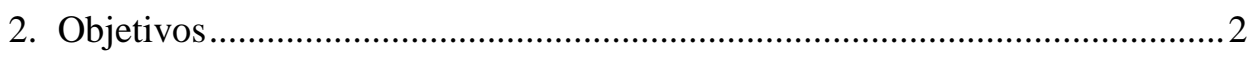

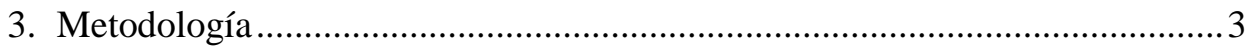

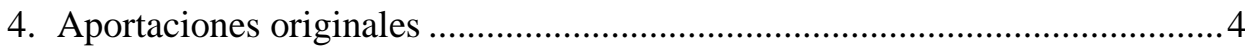

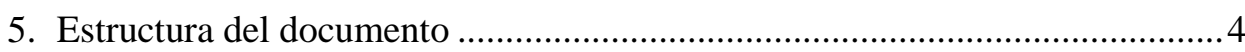

CAPÍTULO 1. PANORAMA CIENTÍFICO......................................................7

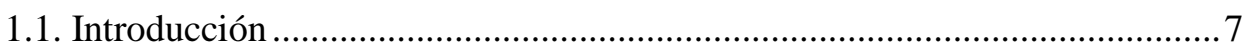

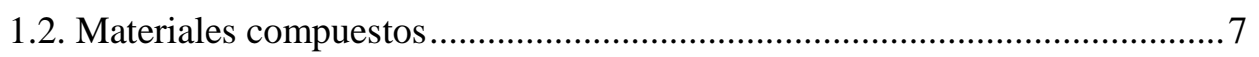

1.2.1. Materiales compuestos de matriz polimérica reforzados con fibras ..................... 8

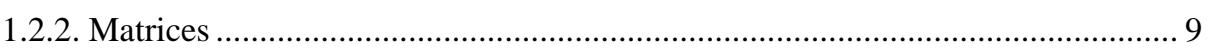

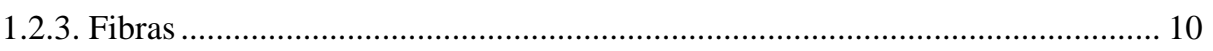

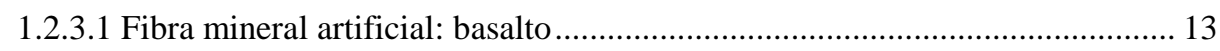

1.3. Procesos de fabricación de materiales compuestos...................................... 16

1.3.1. Proceso RTM (Resin Transfer Molding) ...................................................... 19

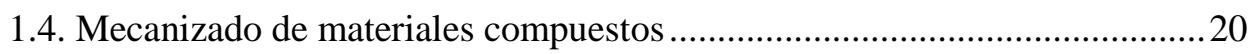

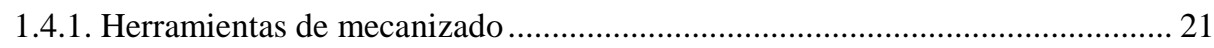

1.5. Estado del arte del fresado de materiales compuestos ................................2 23

1.5.1. Materiales, fabricación de laminados y operaciones de fresado ........................ 24

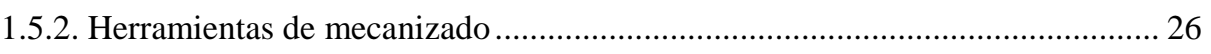




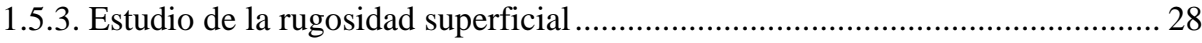

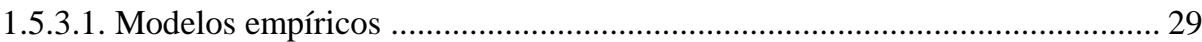

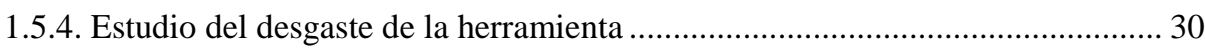

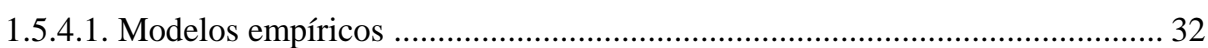

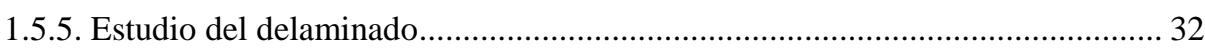

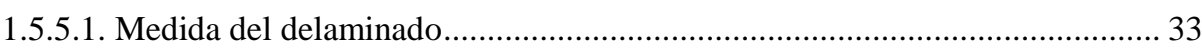

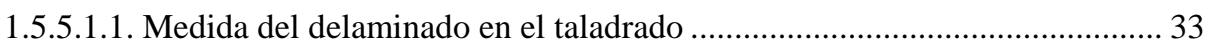

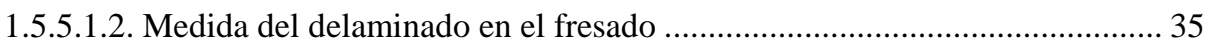

1.5.5.2. Parámetros que influyen en el delaminado ................................................... 36

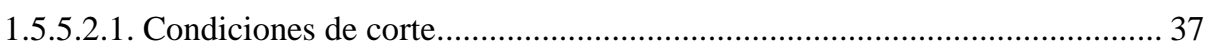

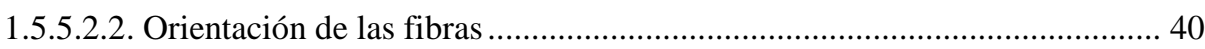

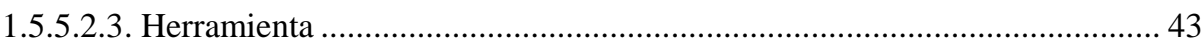

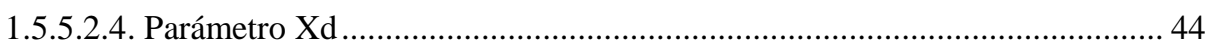

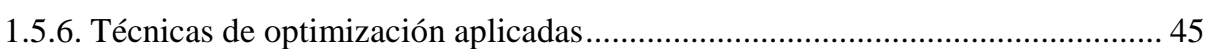

\section{CAPÍTULO 2. MATERIALES Y MÉTODOS ...................................................47}

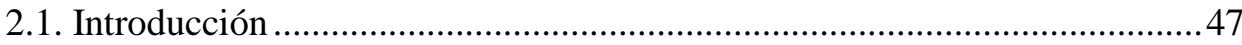

2.2. Fabricación de los laminados por RTM .....................................................48

2.3. Mecanizado de los laminados ......................................................................50

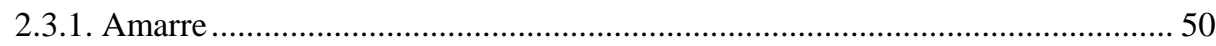

2.3.2. Selección de la herramienta de corte .............................................................. 52

2.4. Planteamiento de los experimentos.............................................................53

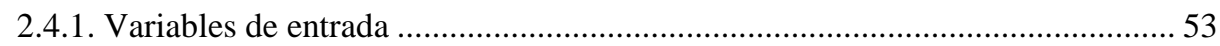

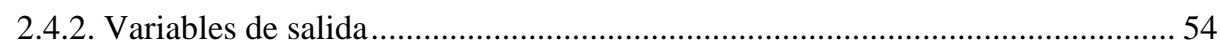

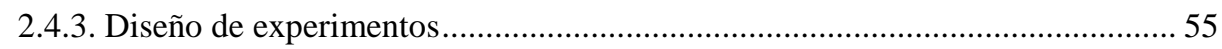

2.5. Procedimiento de medida de las variables de salida ...................................56

2.5.1. Procedimiento de medida de la rugosidad superficial ........................................ 56

2.5.2. Procedimiento de medida del desgaste de la herramienta................................ 58

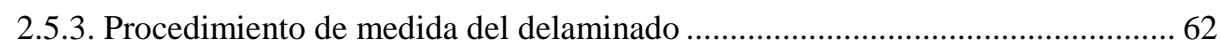

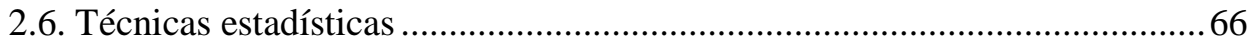

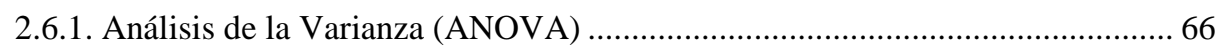

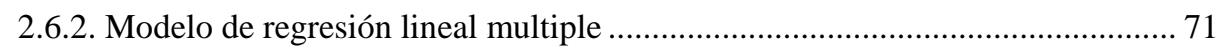

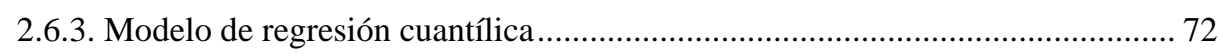




\section{CAPÍTULO 3. ANÁLISIS DE LA RUGOSIDAD SUPERFICIAL Y} EL DESGASTE DE LA HERRAMIENTA .......................................75

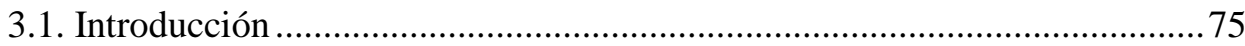

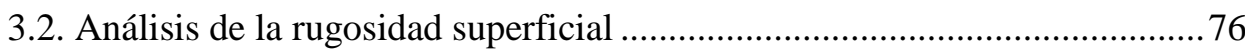

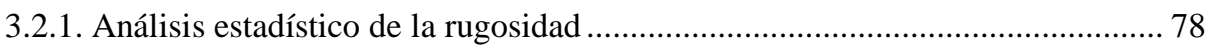

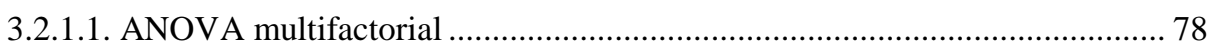

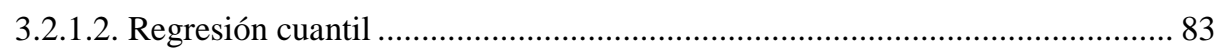

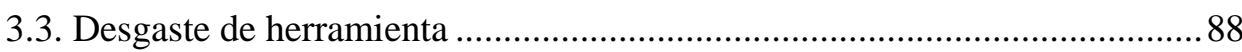

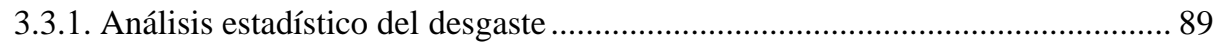

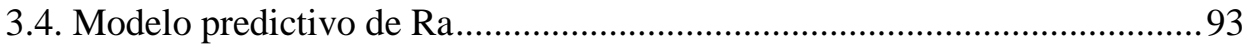

CAPÍTULO 4. ANÁLISIS DEL DELAMINADO ...........................................97

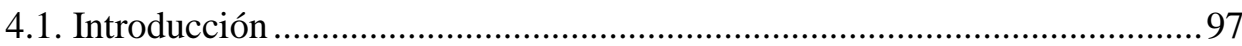

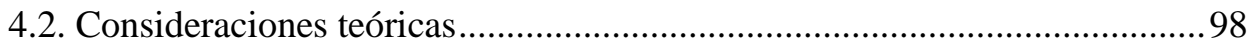

4.2.1. Referencias de posicionamiento angular ......................................................... 98

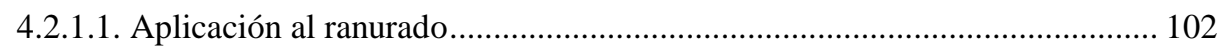

4.2.2. Orientación de la trayectoria de corte respecto al tejido: efecto en el

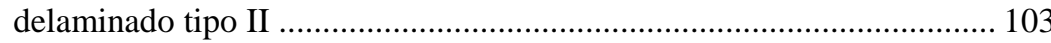

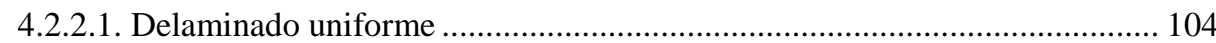

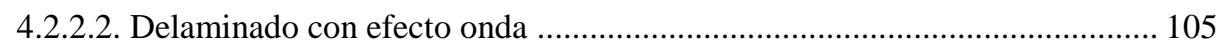

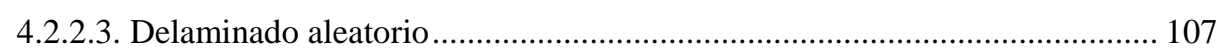

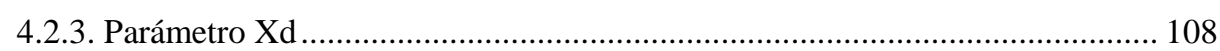

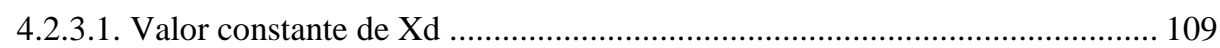

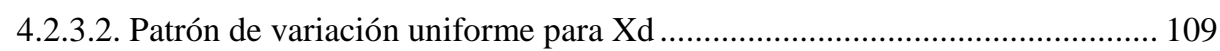

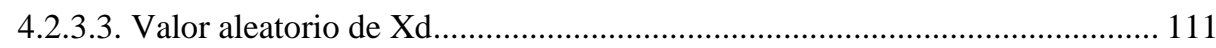

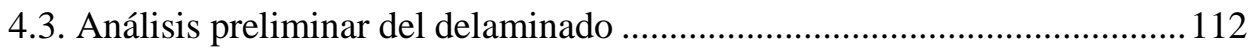

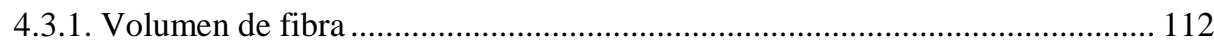

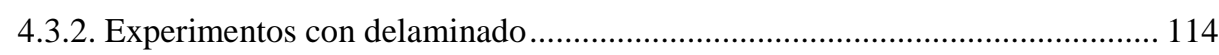

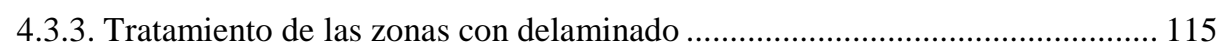

4.3.4. Análisis del delaminado tipo I y tipo II en un tejido bidimensional ................. 119

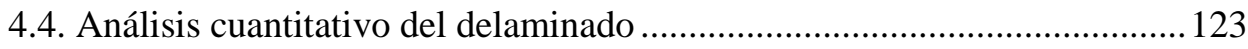




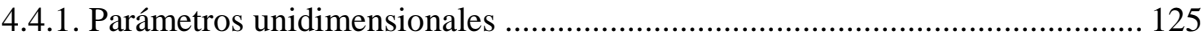

4.4.1.1. Parámetro de delaminado medio (Wa) ........................................................... 125

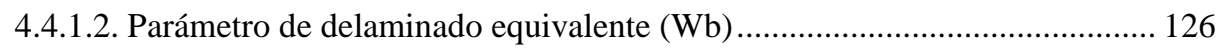

4.4.1.2.1. Valor máximo teórico (Wmax) del parámetro de delaminado equivalente

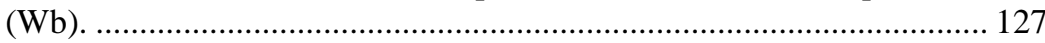

4.4.1.3. Factor de delaminado (Fdel) ................................................................ 128

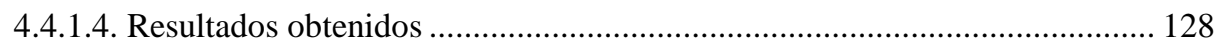

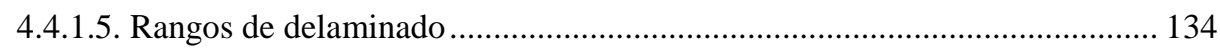

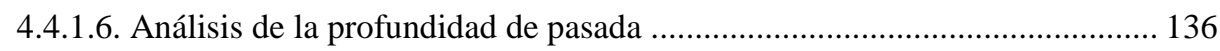

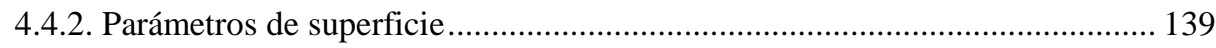

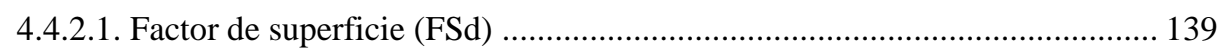

4.4.2.1.1 Valor máximo teórico de la superficie delaminada (Sdmax) ..................... 140

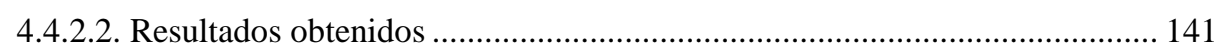

4.4.2.3. Rangos de delaminado para el factor de superficie....................................... 144

4.5. Análisis estadístico................................................................ 145

4.5.1. Delaminado Tipo I...................................................................................... 145

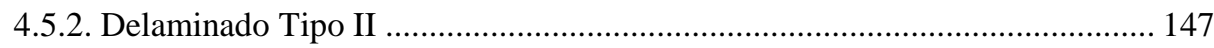

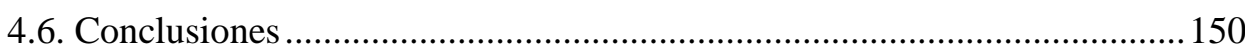

\section{CAPÍTULO 5. CONCLUSIONES Y TRABAJOS FUTUROS ................. 151}

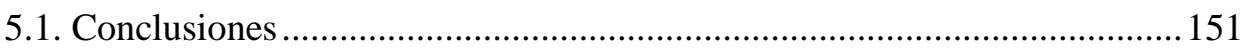

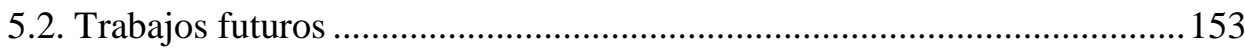

PUBLICACIONES ..................................................................... 155

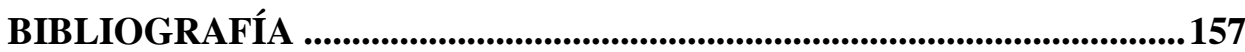

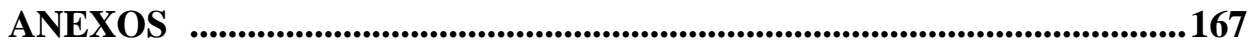

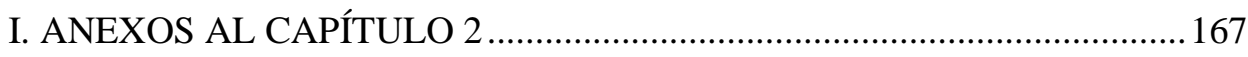

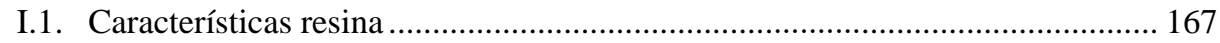

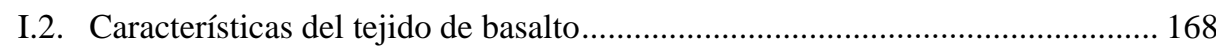

I.3. Especificaciones centro de mecanizado. KONDIA B-500 …......................... 170 


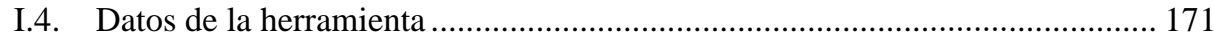

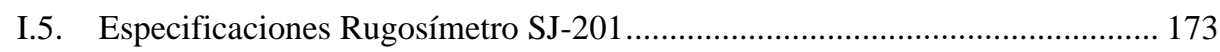

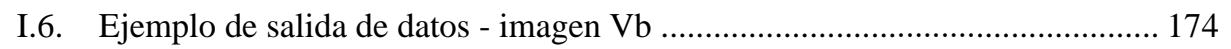

II. ANEXOS AL CAPÍTULO 3 ............................................................. 176

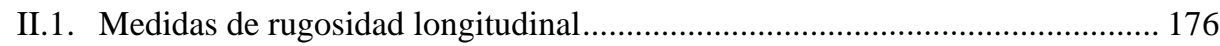

II.2. Gráficos de rugosidad superficial ............................................................... 184

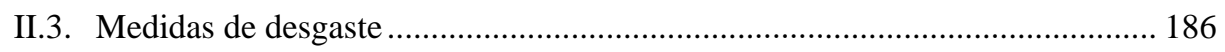

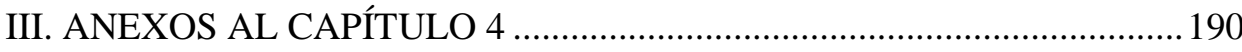

III.1. Ejemplo datos delaminado ........................................................................... 190 


\section{ÍNDICE DE FIGURAS}

\section{Capítulo 1}

Figura 1.1. Ejemplo de componentes: tejido (refuerzo) + resina (matriz polimérica). 8

Figura 1.2. Ilustración esquemática de los métodos de refuerzo utilizados para

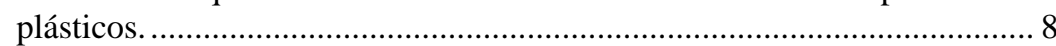

Figura 1.3. Aplicaciones de materiales compuestos de matriz polimérica. .................. 9

Figura 1.4. Sección transversal de una tabla de Windsurf hecha de materiales compuestos. ................................................................................... 9

Figura 1.5. Tejido de refuerzo de fibra bidireccional y refuerzo roving.................... 12

Figura 1.6. Trama y urdimbre de un tejido bidireccional. ...................................... 12

Figura 1.7. Diferentes configuraciones de tejidos de refuerzo. ................................ 13

Figura 1.8. Coste típico (libra $/ \mathrm{m}^{2}$ ) de diferentes tejidos de refuerzo. (Sandvik C2920)

Figura 1.9. Composición química del Basalto......................................................... 15

Figura 1.10. Gráfico comparativo de las propiedades mecánicas de diferentes fibras de

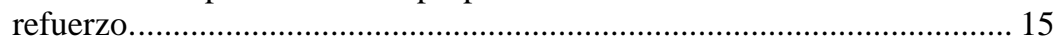

Figura 1.11. Clasificación por tipo de molde utilizado.......................................... 17

Figura 1.12. Procesos de conformado por vía seca.................................................... 18

Figura 1.13. Esquema del Proceso RTM.............................................................. 19

Figura 1.14. Contorneado o "edge trimming". ....................................................... 20

Figura 1.15. Herramientas de fresado para materiales compuestos........................... 21

Figura 1.16. Zonas de desgaste de la herramienta. ................................................... 22

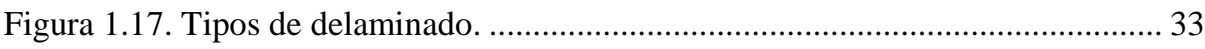

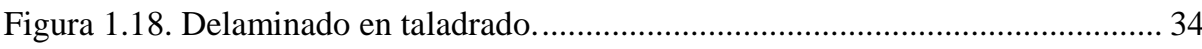

Figura 1.19. Factor de delaminado. ........................................................................ 34

Figura 1.20. Posibles casos de evaluación del delaminado. (a) delaminación uniforme.

(b) fibras largas con delaminación no uniforme....................................... 34

Figura 1.21. Factor de delaminado en el ranurado.................................................. 36

Figura 1.22. Influencia de las condiciones de corte en el ranurado. ........................... 38

Figura 1.23. Influencia de las condiciones de corte en el contorneado........................ 40

Figura 1.24. Ángulos relacionados con la orientación de la fibra en fresado. ............. 41

Figura 1.25. Zona crítica de inicio del delaminado. ................................................... 42

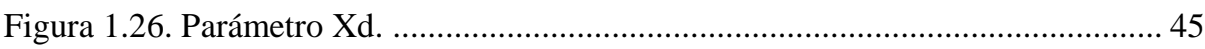

Figura 1.27. Regiones en la capa superior del laminado. ......................................... 45 


\section{Capítulo 2}

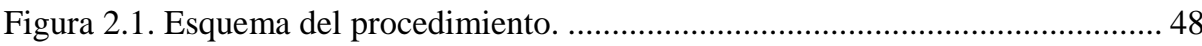

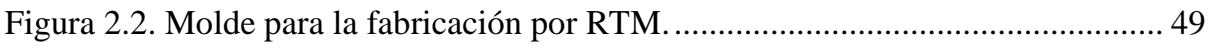

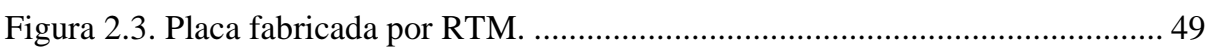

Figura 2.4. Montaje de equipos para la fabricación de los laminados. ......................... 49

Figura 2.5. Esquema del proceso de mecanizado. ................................................... 50

Figura 2.6. Elementos de sujeción para el amarre de los laminados............................ 51

Figura 2.7. (a) Máximo voladizo del laminado (b) Galga de control. .......................... 51

Figura 2.8. Esquema del fresado periférico y amarre en la máquina........................... 51

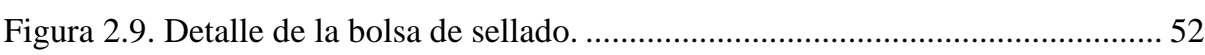

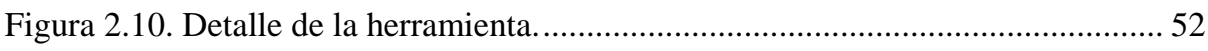

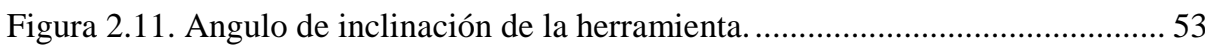

Figura 2.12. (a) Rugosímetro (b) Banco de medida de rugosidad. .............................. 56

Figura 2.13. Posiciones de medida de rugosidad sobre el laminado............................ 57

Figura 2.14. (a) Puntos de medida en el espesor de la placa. (b) Posición palpador. 57

Figura 2.15. Elementos del banco de medida de desgaste......................................... 58

Figura 2.16. Banco de medida del desgaste de la herramienta. ................................... 59

Figura 2.17. Ventana de registro de datos y ventana de imagen................................... 60

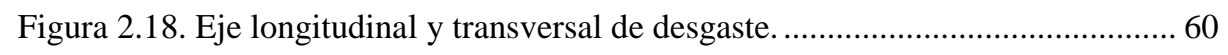

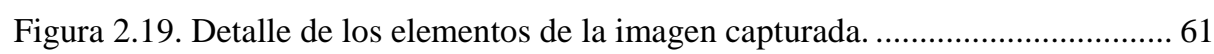

Figura 2.20. Detalle del borde fresado de una pieza con delaminado............................ 62

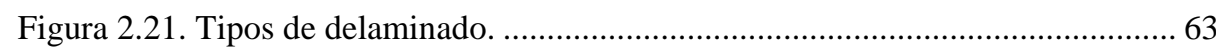

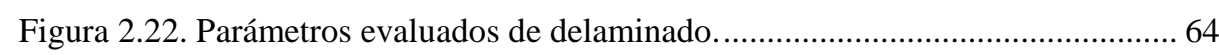

Figura 2.23. Ejemplo de numeración de mechas en la longitud evaluada .................... 65

Figura 2.24. Ejemplo Mecha 4 parámetros delaminado (a) tipo I (b) tipo II

(c) histograma tipo I

\section{Capítulo 3}

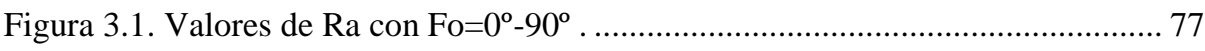

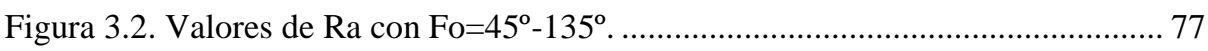

Figura 3.3. Diagrama Box-Whisker para Ra y los diferentes Tc................................. 79

Figura 3.4. Gráficos de análisis de residuos modelo ANOVA Tc=5 minutos............ 81

Figura 3.5. Gráficos de análisis de residuos modelo ANOVA Tc=50 minutos.......... 82

Figura 3.6. Gráficos de análisis de residuos modelo ANOVA Tc $=80$ minutos. ......... 82 
Figura 3.7. Histograma, función de densidad empírica de Ra.................................. 84

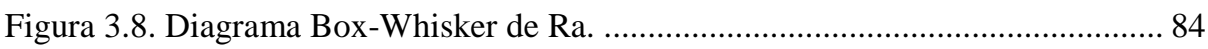

Figura 3.9. Cuantiles Ra vs parámetros de mecanizado y fibra.................................. 86

Figura 3.10. Rugosidad mediana observada, estimada e intervalo de confianza

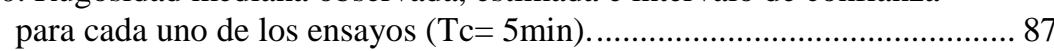

Figura 3.11. Rugosidad mediana observada, estimada e intervalo de confianza

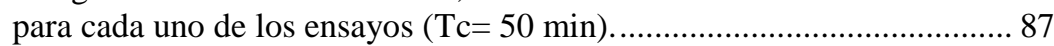

Figura 3.12. Rugosidad mediana observada, estimada e intervalo de confianza

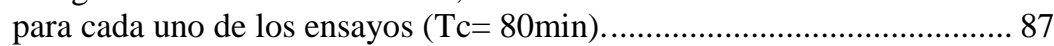

Figura 3.13. Desgaste de flanco $(\mathrm{Vb})$ frente a tiempo de corte $(\mathrm{Tc})$.......................... 89

Figura 3.14. Desgaste observado (Vb) frente a tiempo de corte (Tc)...................... 90

Figura 3.15. Gráficos de los residuos. Modelo de regresión lineal para la predicción de la Rugosidad media.

Figura 3.16. Rugosidad (Ra) observada y predicha con el modelo de regresión frente al desgaste $(\mathrm{Vb})$.

\section{Capítulo 4}

Figura 4.1. Ángulo de empañe $\varphi$ .99

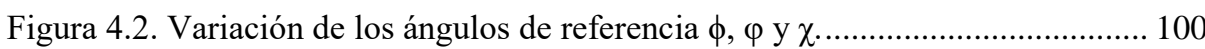

Figura 4.3. Ángulos de referencia al inicio y al final del corte............................... 101

Figura 4.4. Ángulos de referencia en el mecanizado de una ranura. ........................ 103

Figura 4.5. Delaminado tipo II uniforme en orientaciones principales..................... 104

Figura 4.6. Esquema del delaminado tipo II: Uniforme. ......................................... 105

Figura 4.7. Aspecto del efecto onda de delaminado tipo II. ..................................... 105

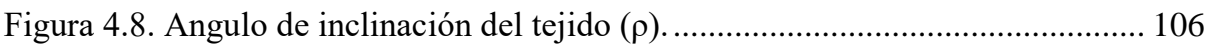

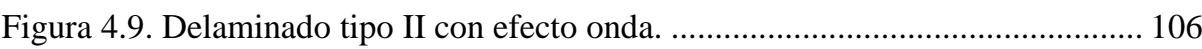

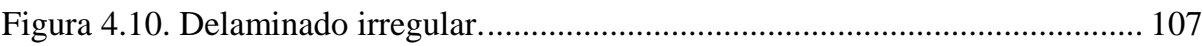

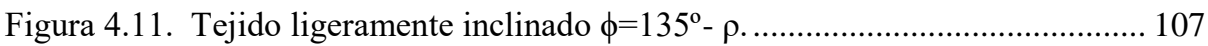

Figura 4.12. Dirección de las mechas delaminadas en un tejido bidireccional.......... 108

Figura 4.13. Delaminado tipo II aleatorio............................................................. 108

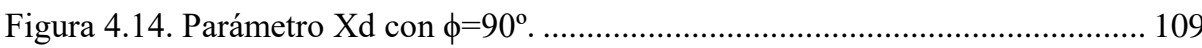

Figura 4.15. Variación de Xd entre mechas contiguas a $90^{\circ}$.................................... 110

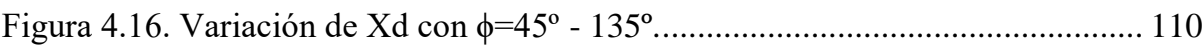

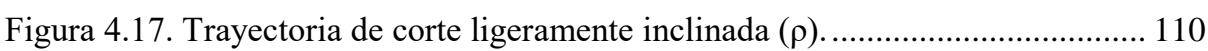

Figura 4.18. Variación de Xd y del delaminado....................................................... 111 
Figura 4.19. Variación de Xd para tejido $45^{\circ}-135^{\circ}$ inclinado ligeramente $\rho$. ........... 111

Figura 4.20. Aspecto de la superficie mecanizada................................................... 112

Figura 4.21. Detalle de la capa de resina superficial. ............................................ 113

Figura 4.22. Disposición de las mechas de tejido en la capa superficial de la pieza. 113

Figura 4.23. Delaminado con distribución irregular de la fibra y la resina................ 114

Figura 4.24. Zona borde delaminado y zona corte limpio en tipo II........................ 117

Figura 4.25. Tejido de basalto utilizado para la fabricación de las piezas ................. 120

Figura 4.26. Detalle de la capa superficial de tejido que sufre delaminado.............. 120

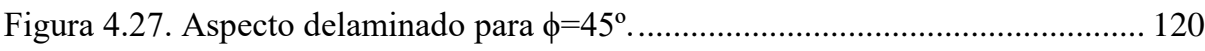

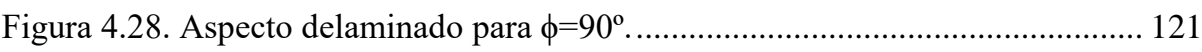

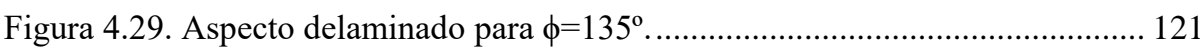

Figura 4.30. Superficie delaminada para Fo $0^{\circ}-90^{\circ}$.(a) delaminado en las mechas por encima de la trama $(b, c)$ delaminado en las mechas por encima y por debajo de la trama. .................................................................... 122

Figura 4.31. Numeración de mechas por encima de la trama ................................ 123

Figura 4.32. Numeración de mechas por debajo de la trama ................................... 123

Figura 4.33. Longitud (L) donde se ha evaluado el delaminado............................... 123

Figura 4.34. Ejemplo de medida de las características para delaminado tipo II. ....... 124

Figura 4.35. Parámetro de delaminado medio (Wa) para tipo II. ............................ 125

Figura 4.36. Medida de longitud y superficie delaminada tipo II............................ 126

Figura 4.37. Parámetro de delaminado medio equivalente $(\mathrm{Wb})$. ............................ 126

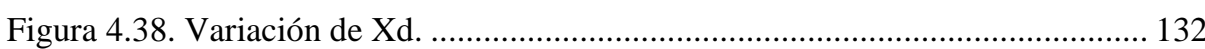

Figura 4.39. Rango del parámetro Wa...................................................................... 135

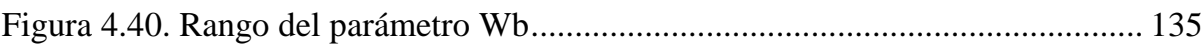

Figura 4.41. Factor de delaminado, Fdel .............................................................. 136

Figura 4.42. Corte transversal del tejido, ap y hmax observada ............................ 137

Figura 4.43. Valor del parámetro hmax sobre el tejido ............................................. 138

Figura 4.44. Delaminado observado en E05 ap=0.5 mm y Tc=80 minutos.............. 138

Figura 4.45. Delaminado tipo I/II: ligero y denso ................................................. 139

Figura 4.46. Cálculo de Sd máxima teórica para delaminado Tipo II. ..................... 141

Figura 4.47. Rango Factor de superficie de delaminado Tipo I (FSd+FSd')............ 144

Figura 4.48. Rango Factor de superficie de delaminado Tipo II (FSd+FSd'). ......... 144 


\section{ÍNDICE DE TABLAS}

\section{Capítulo 1}

Tabla 1.1. Tipos de matrices poliméricas. 10

Tabla 1.2. Tipos de fibras de refuerzo en función de su origen................................... 10

Tabla 1.3. Refuerzos a base de fibras largas. (Michaeli W.) [2] ................................... 11

Tabla 1.4. Compuestos laminados para fresado evaluados en la literatura................. 25

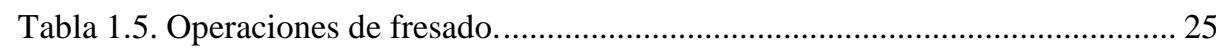

Tabla 1.6. Características de las herramientas utilizadas............................................ 26

Tabla 1.7. Modelos empíricos para rugosidad superficial en función de las condiciones de corte y por tipo de herramienta. ..................................... 29

Tabla 1.8. Modelos empíricos para el desgaste de la herramienta................................ 32

Tabla 1.9. Modelos del delaminado en función de las condiciones de corte. ............... 40

Tabla 1.10. Definición ángulos de orientación de fibra para delaminado...................... 41

\section{Capítulo 2}

Tabla 2.1. Características del material: refuerzo y matriz. 48

Tabla 2.2. Variables de entrada. Niveles definidos del diseño de experimentos. ........ 54

Tabla 2.3. Valores de las variables de entrada por experimento................................. 55

Tabla 2.4. Ejemplo de registro de salida de datos -Medida desgaste E02.................. 61

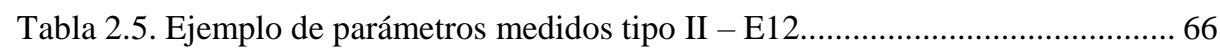

Tabla 2.6. Ejemplo tabla ANOVA dos factores con interacción ................................... 67

Tabla 2.7. Ejemplo tabla ANOVA de un factor de medidas repetidas. ....................... 70

\section{Capítulo 3}

Tabla 3.1. Rugosidad media ( $\mathrm{Ra})$ para distintos tiempos de corte............................ 76

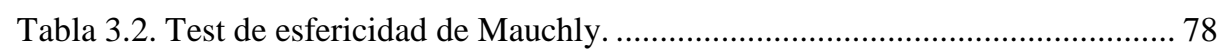

Tabla 3.3. Resultados ANOVA de medidas repetidas con corrección de esfericidad. 78

Tabla 3.4. Tabla ANOVA para Ra para $\mathrm{Tc}=5$ minutos. ......................................... 79

Tabla 3.5. Tabla ANOVA para Ra para $\mathrm{Tc}=50$ minutos. ......................................... 79

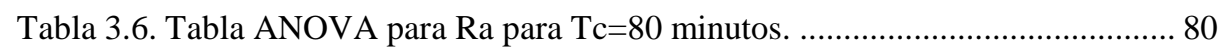

Tabla 3.7. Resultados de la prueba Saphiro-Wilks.................................................. 80

Tabla 3.8. Resultados prueba Barlett..................................................................... 80 


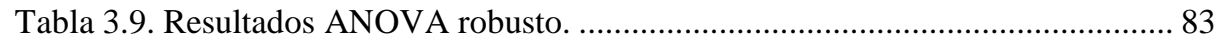

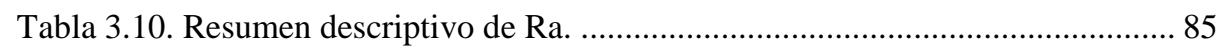

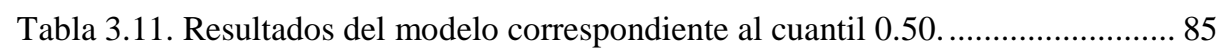

Tabla 3.12. Valor más desfavorable de desgaste por experimento. ............................. 88

Tabla 3.13. Test de esfericidad de Mauchly. ............................................................... 90

Tabla 3.14. Resultados ANOVA medidas repetidas con corrección de esfericidad. .. 90

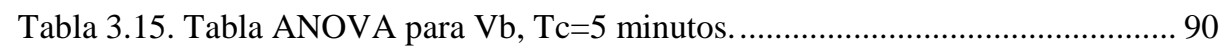

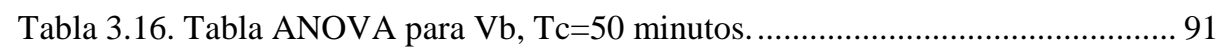

Tabla 3.17. Tabla ANOVA para Vb, Tc=80 minutos.............................................. 91

Tabla 3.18. Resultados test Saphiro-Wilks............................................................... 92

Tabla 3.19. Resultados prueba Barlett. ....................................................................... 92

Tabla 3.20. Resultados ANOVA robusto. .................................................................... 92

Tabla 3.21. Resultados del modelo de regresión de la rugosidad en función del desgaste, el Tc y los parámetros de mecanizado y material. ................... 93

Tabla 3.22. Resultados test Saphiro-Wilks y F-test. Comprobación de supuestos

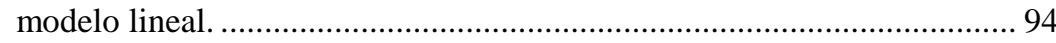

\section{Capítulo 4}

Tabla 4.1. Tipos de delaminado observados.

Tabla 4.2. Zonas con distintos tipos de delaminado ............................................... 117

Tabla 4.3. Valor de los parámetros para el delaminado Tipo I................................. 128

Tabla 4.4. Valor de los parámetros para el delaminado Tipo II............................... 130

Tabla 4.5. Comparación de parámetros Tipo I y Tipo II. .......................................... 133

Tabla 4.6. Experimentos donde considerar los valores "prima" ............................. 133

Tabla 4.7. Comparación experimentos con distintas profundidades de pasada ........ 138

Tabla 4.8. Valores máximos teóricos de delaminado para una mecha y la longitud evaluada.

Tabla 4.9. Parámetros de superficie para delaminado Tipo I................................... 141

Tabla 4.10. Parámetros de superficie para delaminado Tipo II. ................................ 143

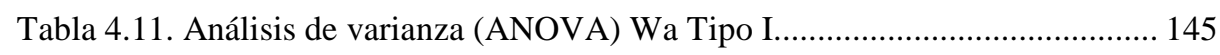

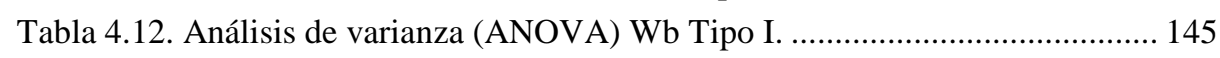

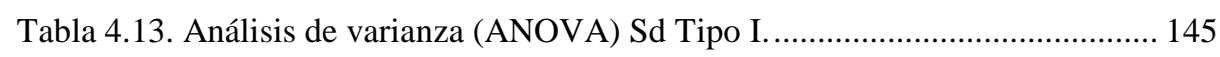

Tabla 4.14. Análisis de varianza (ANOVA) FSd+FSd’ Tipo I.................................. 146

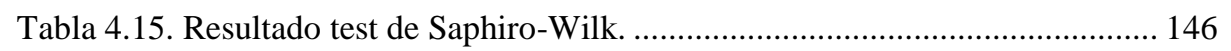

Tabla 4.16. Resultado test de Levene. ................................................................... 147 
Tabla 4.17. Análisis de varianza (ANOVA) robusto delaminado Tipo I.................. 147

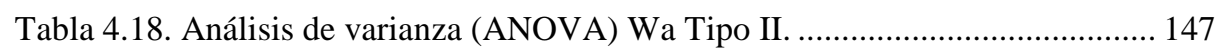

Tabla 4.19. Análisis de varianza (ANOVA) Wb Tipo II.......................................... 148

Tabla 4.20. Análisis de varianza (ANOVA) Sd Tipo II. .......................................... 148

Tabla 4.21. Análisis de varianza (ANOVA) FSd+Fsd' Tipo II............................... 148

Tabla 4.22. Resultado test de Saphiro-Wilk. ............................................................ 149

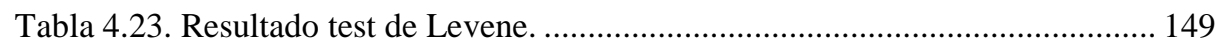

Tabla 4.24. Análisis de varianza (ANOVA) robusto delaminado Tipo II ................. 149 


\section{PRESENTACIÓN DE LA TESIS}

\section{INTRODUCCIÓN}

En los sectores aeronáutico, naval y de automoción, la aplicación de los materiales compuestos de matriz polimérica reforzados con fibras está en auge, debido principalmente a su ligereza, su elevada resistencia mecánica y su resistencia ante la corrosión y a las altas temperaturas.

Los procesos de conformado de estos materiales (moldeo por transferencia, infusión, etc.) permiten obtener piezas con una geometría cercana a la deseada. Tras el curado, se necesitan procesos de mecanizado para eliminar el material sobrante y conseguir las formas y tolerancias requeridas, así como para permitir los procesos de unión entre diferentes piezas. De entre los diferentes procesos de mecanizado, los más utilizados son el taladrado y el fresado y, dentro de este último, las operaciones de ranurado y de contorneado.

Los materiales compuestos reforzados con fibra, debido a su naturaleza heterogénea y su anisotropía, tienen un comportamiento diferente ante el mecanizado que los metálicos. La resistencia mecánica de las fibras abrasivas produce un desgaste rápido de la herramienta, así como un peor acabado superficial y delaminado en las piezas. 
Las investigaciones llevadas a cabo hasta el momento sobre el mecanizado de estos materiales, se han realizado en materiales con refuerzo de fibras de carbono y de vidrio, no existiendo casi ningún estudio sobre fibra de basalto. Las fibras de basalto son una buena alternativa, principalmente, a las fibras de vidrio en cuanto a propiedades y coste. Además, la fibra de basalto es de origen mineral, es completamente inerte, no es tóxica y es buen aislante térmico y eléctrico.

Los trabajos existentes sobre el mecanizado de materiales compuestos se han centrado, sobre todo, en el estudio de los procesos de taladrado y fresado. Estos trabajos evalúan tanto el propio proceso de fabricación, como la calidad obtenida en la pieza a través del estudio de su acabado superficial, las fuerzas generadas durante el corte, las potencias consumidas, el desgaste de la herramienta, el delaminado, etc. Por ello, intentan buscar cuáles son las condiciones de corte más adecuadas, la selección de la herramienta más apropiada y la influencia que tiene el propio material compuesto a mecanizar.

En relación con la calidad obtenida en la pieza, el delaminado es uno de los defectos más importantes, ya que afecta tanto a la precisión dimensional como a la posterior unión entre piezas, pudiendo hacer necesario el reprocesado de la pieza, e incluso provocar su rechazo. En el estudio del delaminado se ha intentado cuantificar este defecto en el proceso de taladrado y de fresado, y en este último la operación de ranurado y contorneado, determinando las condiciones de trabajo más adecuadas para intentar minimizarlo. Sin embargo, en la operación de contorneado en fresado, esta cuantificación no es tan sencilla y aún se están desarrollando trabajos relacionados con este aspecto.

El planteamiento de esta tesis surge con el objetivo de avanzar en el estudio de la operación de contorneado en el fresado de materiales compuestos de materiales reforzados con fibra. Por un lado, se van a estudiar los materiales reforzados con fibra de basalto, de los que prácticamente no hay estudios en este nivel. Por otro lado, para caracterizar mejor este proceso se va a abordar el estudio del desgaste de la herramienta, la medida de la rugosidad superficial $\mathrm{y}$, principalmente el delaminado.

\section{OBJETIVOS}

El objetivo de la tesis consiste en el estudio experimental del fresado de laminados de material compuesto reforzado con fibras de basalto, fabricados mediante moldeo por transferencia de resina (RTM). Para ello, una vez fabricados los laminados, se mecanizarán mediante la operación de contorneado y se evaluará tanto el desgaste de la herramienta como la calidad 
de las piezas obtenidas, ésta última mediante la medida del delaminado y la rugosidad superficial.

Para conseguir este objetivo general, se van a abordar los objetivos parciales que se detallan a continuación:

- Fabricación del material compuesto. Los diferentes laminados se fabricarán mediante RTM, modificando tanto el volumen de fibra como la orientación de la fibra.

- Selección de la herramienta de mecanizado y las condiciones de corte. La selección de la herramienta se hará con el objetivo de obtener una alta productividad, fijando rangos para los parámetros de corte (velocidad, avance y profundidad), de acuerdo a las recomendaciones de los fabricantes.

- Diseño y fabricación de los elementos de medición. Se diseñará y fabricará un banco de medida para posicionar la pieza y evaluar la rugosidad superficial, así como un banco adicional para posicionar la herramienta y medir el desgaste del flanco.

- Desarrollo de procedimientos de medida, software y análisis para la evaluación de la rugosidad superficial, el desgaste de la herramienta y el delaminado.

- Análisis de los parámetros y variables que condicionan el proceso de contorneado de un laminado de material compuesto.

- Aplicación de técnicas estadísticas. Mediante un diseño de experimentos se han determinado los ensayos a realizar. Posteriormente se han realizado análisis estadísticos para ver la influencia de las diferentes variables de entrada en las variables de salida.

\section{METODOLOGÍA}

La metodología llevada a cabo para conseguir los anteriores objetivos es principalmente, una metodología experimental. Durante el trabajo experimental se han fabricado los laminados de material compuesto reforzado con fibra de basalto, variando la orientación de las fibras y el volumen de fibra. Posteriormente, los laminados se han fresado modificando las condiciones de corte y se ha medido para diferentes tiempos de corte el desgaste de la herramienta, la rugosidad superficial y los diferentes tipos de delaminado.

Además de la metodología experimental se incluye una segunda metodología basada en estudios estadísticos, para el análisis de la rugosidad, 
el desgaste de la herramienta, y el delaminado, mostrando la incidencia de las condiciones de corte y las propiedades de los laminados sobre estas variables.

\section{APORTACIONES ORIGINALES}

La presente tesis ha dado lugar a las siguientes aportaciones originales:

- Estudio del fresado de materiales compuestos reforzados con fibra de basalto, ya que prácticamente no existen estudios en la literatura sobre el mecanizado de estos materiales.

- Definición de una metodología experimental que incluye el diseño y la fabricación de los equipos necesarios para estudiar la rugosidad, el desgaste y el delaminado, tras el mecanizado de laminados de material compuesto reforzado con fibras.

- Se han establecido las bases teóricas para describir el fenómeno del delaminado para este tipo de materiales, como las referencias de posicionamiento angular y las formas en que se presenta el delaminado.

- Estudio de la influencia de los parámetros de corte (velocidad de corte, avance por diente y profundidad de pasada) y las características del laminado (volumen de fibra y orientación de fibra) en la rugosidad superficial, el desgaste de la herramienta y el delaminado en el fresado de materiales compuestos reforzados con fibra de basalto.

- Desarrollo de modelos mediante herramientas estadísticas, como el ANOVA o la regresión cuantílica aplicados a la rugosidad superficial y al desgaste.

- Caracterización de los diferentes tipos de delaminado (tipos I y II) mediante la comparación de parámetros unidimensionales y de superficie, evaluando la evolución temporal del delaminado.

\section{ESTRUCTURA DEL DOCUMENTO}

El documento de la tesis se estructura en cinco capítulos, que se resumen a continuación:

- Capítulo 1: Panorama científico. En este capítulo se realiza una introducción a los materiales compuestos reforzados con fibra, junto con una revisión del estado del arte centrada en su mecanizado, y en particular en el proceso de fresado.

- Capítulo 2: Materiales y métodos. En este capítulo se describen los materiales y procesos de fabricación utilizados para la obtención de los 
laminados y su posterior fresado. Seguidamente se describirán los procedimientos de medida de la rugosidad superficial, el desgaste de la herramienta y el delaminado, así como el diseño de experimentos realizado y los análisis estadísticos que se llevarán a cabo.

- Capítulo 3: Análisis de la rugosidad superficial y el desgaste de la herramienta. En este capítulo se van a mostrar todos los resultados obtenidos de la medida de la rugosidad superficial y el desgaste en el flanco de la herramienta, realizando análisis estadísticos para ver la influencia de las variables de entrada en las variables de salida.

- Capítulo 4: Análisis del delaminado. En este capítulo se comienza definiendo aspectos relevantes del delaminado, como los ángulos de corte, etc. Seguidamente se evalúan los diferentes tipos de delaminado, utilizando parámetros unidimensionales y de superficie para su medida, complementando con análisis estadísticos.

- Capítulo 5: Conclusiones y trabajos futuros. En este capítulo se expondrán las principales conclusiones de las tesis y se propondrán los trabajos futuros para continuar en este campo de investigación. 



\section{CAPÍTULO 1. PANORAMA CIENTÍFICO}

\subsection{INTRODUCCIÓN}

En este capítulo se realiza una introducción a los materiales compuestos reforzados con fibra y sus procesos de fabricación. Seguidamente se aborda el proceso de mecanizado de estos materiales y finalmente, se plantea el estado del arte de fresado de materiales compuestos reforzados con fibra.

\subsection{MATERIALES COMPUESTOS}

Un material compuesto está formado por dos o más componentes, químicamente distintos y en fases insolubles, de forma que las propiedades del material final, son superiores a las de cada uno de los componentes por separado. Este tipo de materiales presentan dos elementos principales: la matriz y el refuerzo (Figura 1.1). La matriz configura geométricamente la pieza, da estabilidad y cohesión al conjunto, suele ser flexible y poco resistente, y transmite los esfuerzos al refuerzo, protegiéndolo del deterioro mecánico y químico. Se distinguen tres tipos de matriz, en función del material que la forman: matriz metálica, matriz cerámica y matriz polimérica.

El refuerzo proporciona las propiedades mecánicas al material, como la resistencia y la rigidez, pudiendo distinguir materiales reforzados con partículas, con fibras o materiales estructurales. Además de la matriz y el 
refuerzo, los materiales compuestos poseen otros componentes, como núcleos, cargas y aditivos, que varían en función del método de fabricación y su posterior aplicación (Figura 1.2).
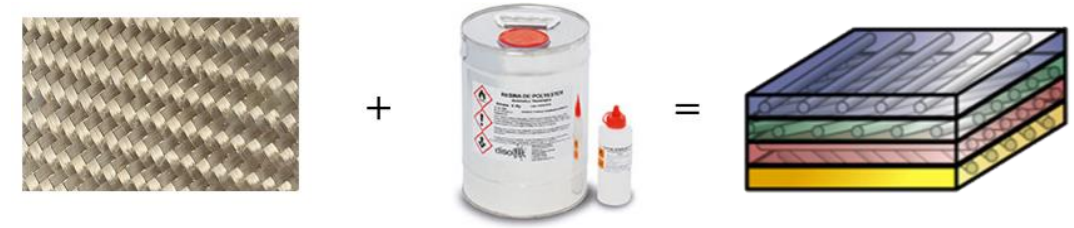

Figura 1.1. Ejemplo de componentes: tejido (refuerzo) + resina (matriz polimérica).

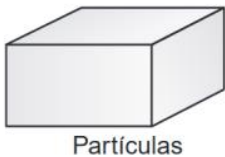

(a)

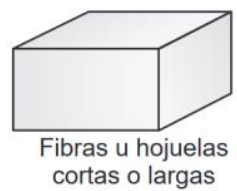

(b)
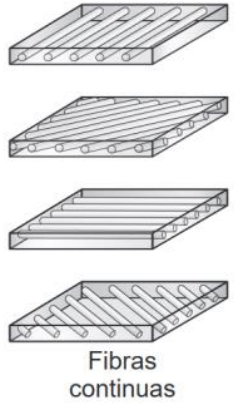

(c)

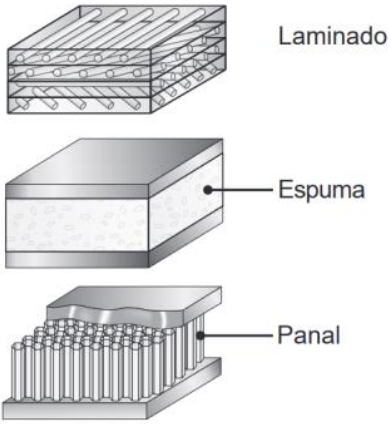

(d)

Figura 1.2. Ilustración esquemática de los métodos de refuerzo utilizados para plásticos [1].

\subsubsection{MATERIALES COMPUESTOS DE MATRIZ POLIMÉRICA REFORZADOS CON FIBRAS}

Uno de los materiales compuestos más empleados es el material polimérico reforzado con fibras, donde la matriz principalmente es una resina de poliéster, vinil éster o epoxi, combinada con distintos tipos de tejidos.

Los materiales compuestos de matriz polimérica reforzados con fibras destacan por su ligereza, su elevada resistencia mecánica y su resistencia ante la corrosión y las altas temperaturas, por lo que son adecuados para diferentes aplicaciones en sectores como el aeronáutico, el de la automoción, el médico, el náutico, etc. (Figuras 1.3 y 1.4). 

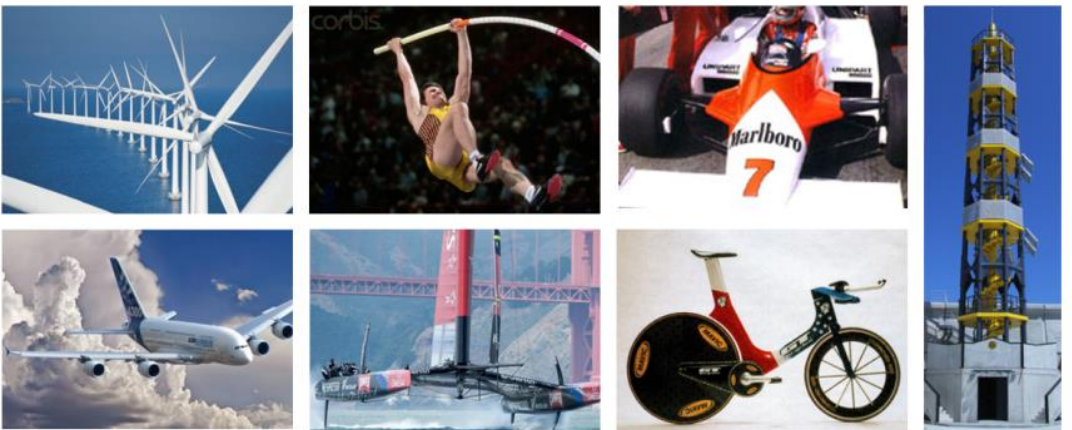

Figura 1.3. Aplicaciones de materiales compuestos de matriz polimérica.

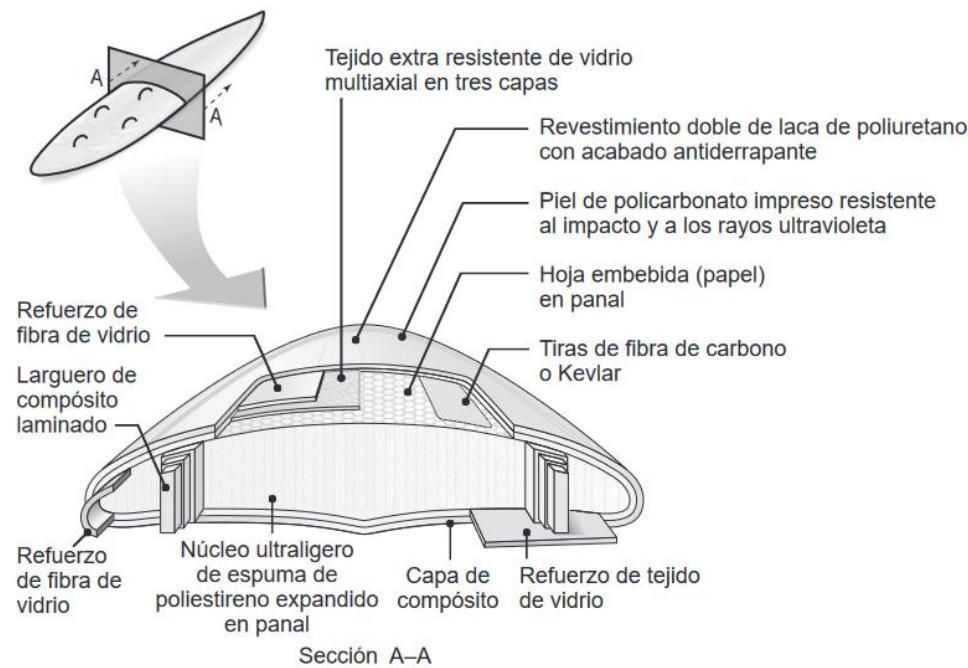

Figura 1.4. Sección transversal de una tabla de Windsurf hecha de materiales compuestos [1].

\subsubsection{MATRICES}

Las matrices poliméricas suelen ser termoplásticas o termoestables (Tabla 1.1). Las resinas termoestables son las más utilizadas en los materiales compuestos de altas prestaciones. Estas resinas se presentan en forma líquida, y mediante una reacción química denominada curado polimerizan, endurecen y pasan a estado sólido, formando una estructura de red tridimensional, con enlaces covalentes. Al aumentar la temperatura, ya no pueden cambiar de estado sólido a líquido, sino que se produce la degradación y descomposición del polímero. Por el contrario, las resinas termoplásticas presentan enlaces más débiles, pudiendo fundirse o plastificar con un aumento de temperatura. 
Tabla 1.1. Tipos de matrices poliméricas.

\begin{tabular}{ll}
\hline Termoplásticas & Termoestables \\
\hline Polietileno (PE) & Resinas epoxi \\
Polipropileno (PP) & Resinas vinil éster \\
Policarbonato (PC) & Resinas poliéster \\
Policloruro de vinilo (PVC) & Fenólica \\
Copolímeros de estireno (ABS, SAN) & Esteres cianato \\
Polimetacrilato de metilo (PMMA) & Bismaleimidas \\
Poliestireno (PS) & Polimidas \\
Poliamidas (PA) & Polieteramida \\
Peek & $\ldots$ \\
\hline
\end{tabular}

Entre las matrices termoestables, la resina epoxi es superior en dureza a la de poliéster, y puede operar a temperaturas más altas, tiene buena adherencia al refuerzo y baja contracción durante la polimerización o curado, siendo especialmente resistente a los ataques de álcali. Esto permite moldeos de alta calidad, con buenas tolerancias dimensionales.

\subsubsection{FIBRAS}

Las fibras de refuerzo que conforman este tipo de materiales compuestos pueden clasificarse en función de diferentes criterios. Los más comunes son los que las clasifican en función de su origen y de la tipología de la fibra (Tabla $1.2)$.

Tabla 1.2. Tipos de fibras de refuerzo en función de su origen.

\begin{tabular}{lll}
\hline Natural (en la naturaleza) & Sintético (origen químico) & Artificial (origen natural) \\
\hline $\begin{array}{l}\text { Vegetal } \\
\text { (algodón, yute) }\end{array}$ & $\begin{array}{c}\text { Orgánicas } \\
\text { (poliamidas, poliésteres, }\end{array}$ & $\begin{array}{l}\text { Orgánicas } \\
\text { (celulósicas) }\end{array}$ \\
aramida) & Inorgánicas / fibras \\
(seda, lana) & Inorgánica & minerales artificiales (FMA): \\
Mineral & (carbono) & (vidrio, basalto, \\
(amianto) & & metálicas, cerámicas) \\
\hline
\end{tabular}

En las aplicaciones industriales, las fibras más utilizadas son las de vidrio y las de carbono. La fibra de vidrio utilizada para los tejidos de refuerzo, está formada por una base de sílice $\left(\mathrm{SiO}_{2}\right)$ y al menos dos silicatos (uno alcalino y otro alcalinotérreo), que se extruyen dando lugar a filamentos de vidrio entre 5 y 24 micras de diámetro. El material que se obtiene es flexible, resistente al calor y a muchos productos químicos, ligero, buen aislante eléctrico y económico. Aunque no es tan fuerte o tan rígida como la fibra de carbono, es 
mucho más barata y mucho menos frágil. De entre los tipos de fibra de vidrio que existen, el más utilizado es el vidrio-E, que posee buenas propiedades eléctricas y trabaja como aislante eléctrico. El material compuesto reforzado con fibra de vidrio se le conoce como GFRP (Glass Fiber Reinforced Polymer).

La fibra de carbono se obtiene, principalmente, a partir del poliacrilonitrilo (PAN) mediante pirólisis de precursores. Cada fibra de carbono es la unión de múltiples filamentos de carbono. Como propiedades fundamentales hay que destacar su alta resistencia, al poseer un módulo de elasticidad elevado, y su baja densidad, si se compara con el acero. También presenta un bajo coeficiente de expansión térmica y baja abrasión, elevada resistencia a la corrosión y alta conductividad eléctrica. El material compuesto con fibra de carbono se le conoce como CFRP (Carbon Fiber Reinforced Polymer).

En relación a la tipología de las fibras, se pueden distinguir diferentes tipos como los mat de hilos, roving, y tejidos unidireccionales, bidireccionales (woven) y tridimensionales (Tabla 1.3 y Figura 1.5). Los mat de hilos están formados por hilos cortados, entrelazados caóticamente, aglomerados y prensados, unidos mediante una resina. Son menos resistentes que los tejidos $y$, cortados en una dirección al azar, presentan la misma resistencia en todas las direcciones (random mat). Los roving están formados por hilos de fibra unidos en haces $\mathrm{o}$ enrollados y son unidireccionales. Los tejidos unidireccionales tienen la mayoría de sus fibras dispuestas en una única dirección, mientras que los tejidos bidireccionales están formados por fibras que se entrecruzan perpendicularmente. En los tejidos bidireccionales se puede distinguir entre la trama y la urdimbre (Figura 1.6). La urdimbre son las fibras longitudinales (en sentido del rollo) y la trama las perpendiculares. Las direcciones resistentes del material compuesto se suelen orientar en las direcciones de la trama y la urdimbre $\left(0^{\circ}\right.$ y $\left.90^{\circ}\right)$.

Tabla 1.3. Refuerzos a base de fibras largas [2].

\begin{tabular}{ll}
\multicolumn{1}{c}{ Tipos de refuerzo } & $\begin{array}{c}\text { Dirección preferente } \\
\text { del esfuerzo }\end{array}$ \\
\hline (Meching de vidrio textil de filamentos de vidrio & Vidrio textil cortado \\
\hline
\end{tabular}




\begin{tabular}{lll}
\hline \multicolumn{1}{c}{ Tipos de refuerzo } & $\begin{array}{c}\text { Dirección preferente } \\
\text { del esfuerzo }\end{array}$ \\
\hline & Tejido de filamentos de vidrio textil & $\begin{array}{l}\text { Tejido unidireccional a base de filamento } \\
\text { de vidrio, o hebra de vidrio corta }\end{array}$ \\
\hline & $\begin{array}{l}\text { Lana de vidrio, hecha con filamentos de } \\
\text { vidrio, con fibra de vidrio corta, o con } \\
\text { fibras sintéticas }\end{array}$ & No aplicable \\
\hline
\end{tabular}
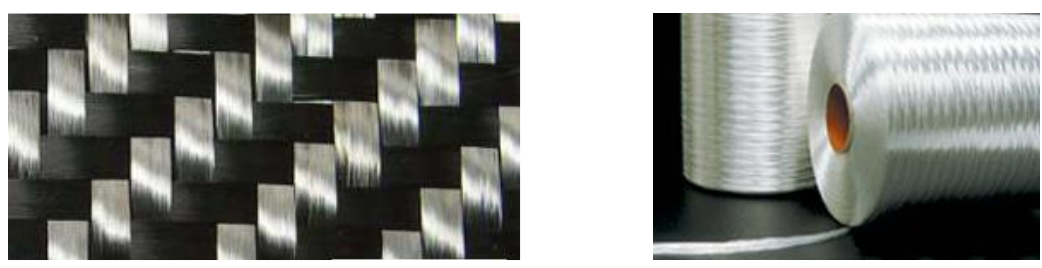

Figura 1.5. Tejido de refuerzo de fibra bidireccional y refuerzo roving.
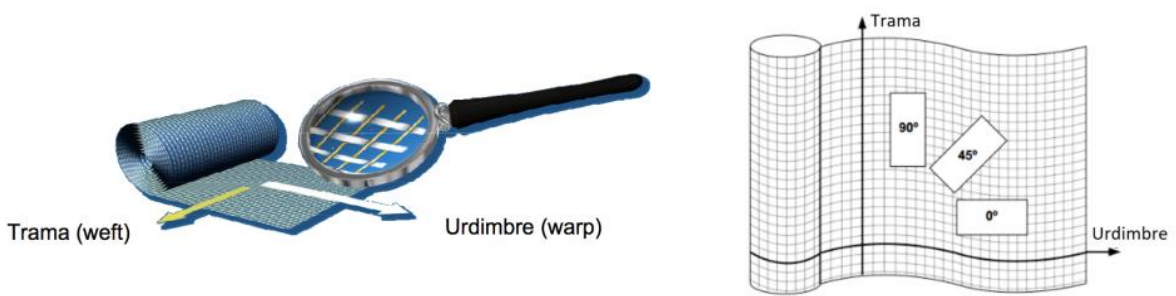

Figura 1.6. Trama y urdimbre de un tejido bidireccional [1].

Según la forma en que se entrecruzan los hilos, los tejidos tienen diferentes denominaciones: tafetán, sarga, satén, etc. (Figura 1.7). También existen tejidos tridimensionales, con fibras dispuestas en los tres ejes, pero son mucho menos utilizados.
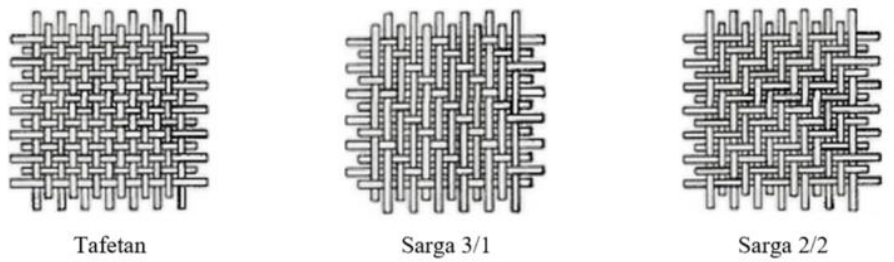

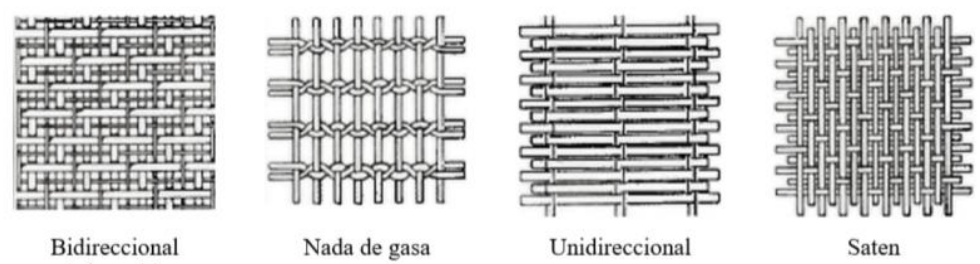

Figura 1.7. Diferentes configuraciones de tejidos de refuerzo [3].

Las propiedades mecánicas y físicas de un plástico reforzado dependen del tipo, forma y orientación del tejido de refuerzo, la longitud de la fibra y del porcentaje de volumen de fibras de refuerzo respecto al contenido de resina.

Las fibras cortas son menos efectivas que las largas y sus propiedades se ven fuertemente afectadas por la temperatura y por el tiempo durante el que se aplica el esfuerzo. Las fibras largas transmiten mejor la fuerza a través de la matriz, por lo que se utilizan en aplicaciones más críticas, en especial a altas temperaturas. Por lo general, se obtiene una rigidez y una resistencia más alta en la pieza fabricada, cuando las fibras se alinean en la dirección de los esfuerzos que debe soportar. Por ejemplo, cuando una pieza se refuerza unidireccionalmente, las propiedades transversales a la dirección principal del refuerzo, son mucho más bajas que las propiedades longitudinales. Por ello, desde el punto de vista funcional, a una pieza de plástico reforzado se le debe proporcionar un refuerzo óptimo, que consiste en elegir el tipo y la orientación de la fibra más conveniente para que sea capaz de soportar los esfuerzos a los que va a estar sometida.

\subsubsection{Fibra mineral artificial: basalto}

En las últimas décadas se han comenzado a utilizar fibras de origen natural como refuerzo en los materiales compuestos, para aumentar la sostenibilidad de estos materiales [4-5]. Las fibras de origen vegetal (lino, cáñamo, coco, yute) presentan una buena biodegradabilidad, lo que atenúa su impacto en el medio ambiente, y tienen un bajo coste de fabricación. Como desventaja principal, hay que señalar que no tienen una buena resistencia mecánica y son sensibles a la temperatura y la humedad, por lo que la adherencia entre ellas y la matriz no suele ser buena [6]. 
Las fibras artificiales de origen mineral, entre las que se encuentra el basalto, son una alternativa a la fibra de vidrio, en cuanto a propiedades y coste (Figura 1.8) [7].

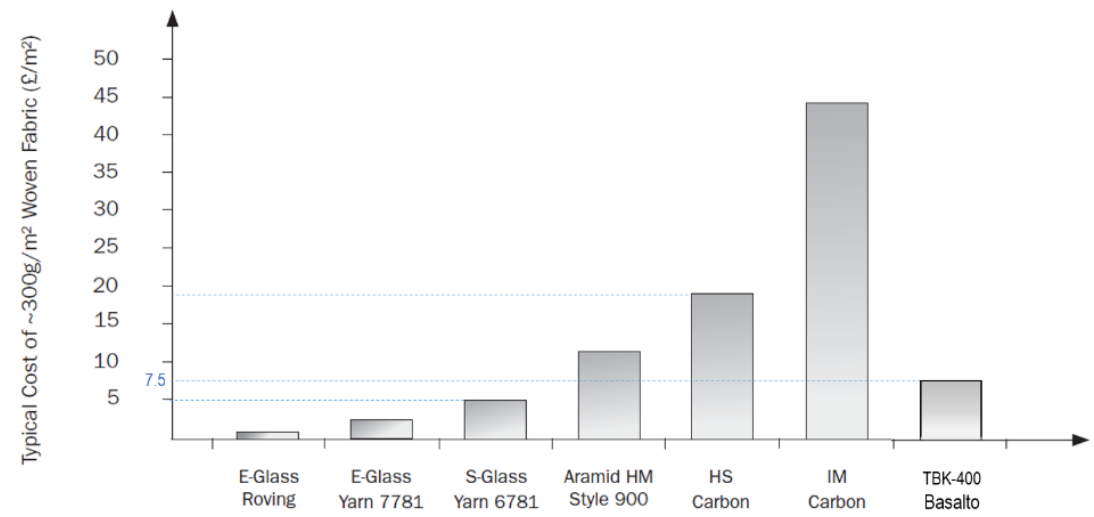

Figura 1.8. Coste (libra/m²) de diferentes tejidos de refuerzo [8].

El basalto es una roca volcánica, que se encuentra abundantemente en la naturaleza, de la cual se obtienen las fibras de basalto fundiendo estas rocas y realizando un proceso de extrusión. La tecnología para obtener estas fibras es muy similar a la utilizada para obtener las fibras de vidrio, pero menos costosa ya que no son necesarios aditivos. La composición del basalto es similar al vidrio (Figura 1.9), siendo sus principales componentes $\mathrm{SiO}_{2}$ y $\mathrm{Al}_{2} \mathrm{O}_{3}$ [9].

La fibra de basalto es completamente inerte, no es tóxica, es un material buen aislante térmico y eléctrico, y tiene propiedades que hacen que su comportamiento sea mejor que el de la fibra de vidrio y ligeramente inferior a la fibra de carbono [10-11]. Presenta muy buena resistencia al fuego y a las altas temperaturas, siendo un material considerado eco-friendly [12].

Las fibras de basalto presentan también otras ventajas competitivas como la amortiguación del ruido y resistencia química y a la fricción. Sin embargo, posee algunas desventajas, como que las propiedades de las fibras de basalto dependen de la calidad y de la composición de las rocas utilizadas como materia prima y que, en ocasiones, presenta poca adhesión entre la fibra y la matriz provocada por el proceso de extrusión necesario para su fabricación [1315]. En la Figura 1.10 se puede observar una comparativa de las propiedades de las fibras más usuales utilizadas como refuerzo en los materiales compuestos [9]. 


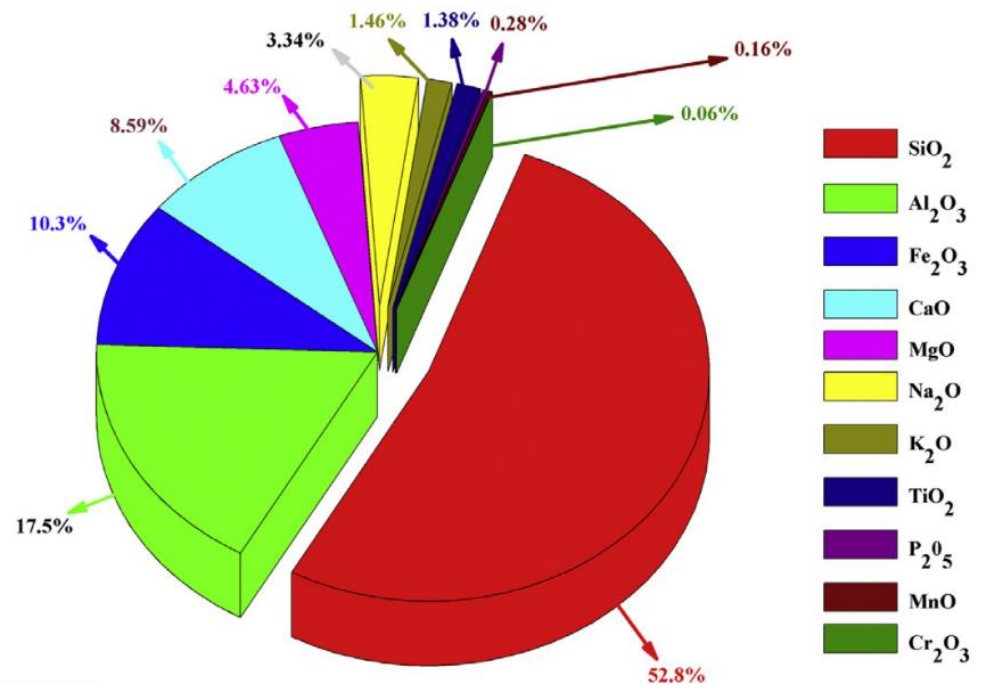

Figura 1.9. Composición química del Basalto [9].

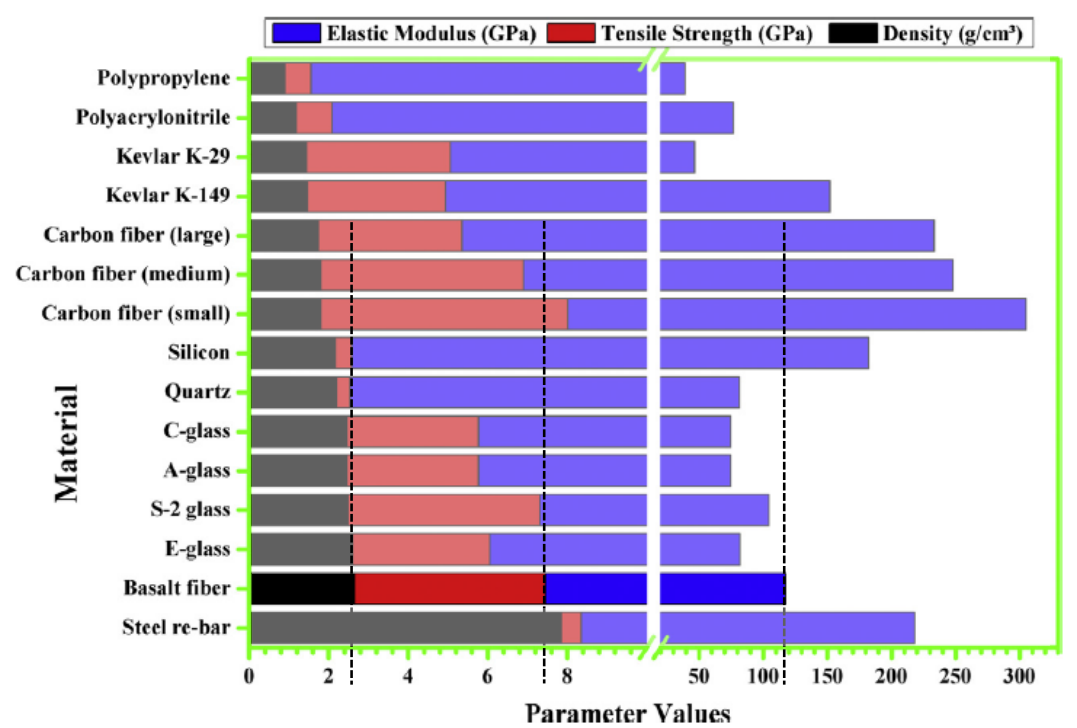

Figura 1.10. Gráfico comparativo de las propiedades mecánicas de diferentes fibras de refuerzo [9] 
Por todas estas características y con el desarrollo que se ha realizado hasta el momento, los materiales compuestos con fibra de basalto son adecuados y se pueden utilizar en las siguientes aplicaciones [5, 16-20]:

- Automoción: carrocerías, recubrimientos internos, pastillas de freno, embragues, camiones frigoríficos.

- Construcción: hormigón reforzado con fibra, estructuras, panelados de interiores, revestimientos, tuberías, depósitos de gas a presión.

- Deportes: esquíes, bicicletas, kayaks, drones, raquetas de tenis, palos de golf, cañas de pescar.

- Náutica: cascos de embarcación, mástiles, aparejos.

- Energía: palas de aerogeneradores, turbinas.

- Aeroespacial: fuselaje, alas, estabilizadores, timón, satélites.

- Industria eléctrica: Circuitos impresos.

- Militar: carros blindados, chalecos antibalas, armamento, cascos.

\subsection{PROCESOS DE FABRICACIÓN DE MATERIALES COMPUESTOS REFORZADOS CON FIBRA}

Existen diferentes procesos de fabricación de materiales poliméricos compuestos que han ido desarrollándose con el paso del tiempo en función de las características de las piezas a fabricar [21]. Las fases de preparación de la fabricación de un material compuesto son: el recorte del tejido adaptado a la geometría de la pieza, la impregnación del tejido con el material polimérico, la polimerización de la resina o proceso de curado y, por último, la extracción o desmoldeo de la pieza. El orden y la forma en el que la matriz y la fibra son mezclados pueden ser diferentes dependiendo del proceso de conformado utilizado. En la fabricación de materiales compuestos, un diseño de proceso donde cada una de estas fases esté perfectamente definida y sea independiente de las otras, permite minimizar los errores de fabricación.

Los procesos de fabricación de materiales compuestos se suelen clasificar en dos grandes familias: procesos de molde abierto y procesos de molde cerrado [3], como muestra la Figura 1.11.

En los procesos de molde abierto se utiliza un semimolde inferior donde se colocan las preformas de tejido y el laminado está expuesto a la atmósfera durante el proceso de fabricación del composite. El proceso es más rápido de 
implementar y más barato, pero se tiene menos control sobre las propiedades de las piezas fabricadas.

En los procesos de molde cerrado, el laminado se fabrica dentro de un molde de dos partes o utilizando una bolsa de vacío, donde la impregnación y el curado se produce con el molde cerrado. Estos procesos son de mayor coste, pero se obtienen piezas de mejor calidad, con un elevado control del acabado y de las propiedades del producto. Se pueden realizar piezas complejas en menos tiempo y de forma más eficiente, reduciendo las operaciones de acabado y con menor emisión de gases y compuestos volátiles.

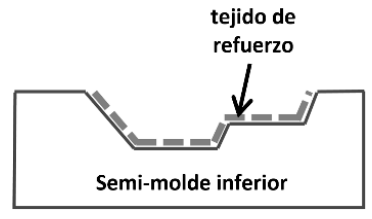

Molde abierto

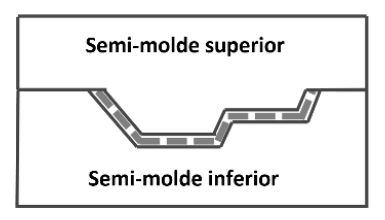

Molde cerrado

Figura 1.11. Clasificación por tipo de molde utilizado.

Los procesos en molde cerrado se pueden clasificar en procesos por vía seca y por vía húmeda. Los procesos de vía húmeda son los que utilizan tejidos preimpregnados, mientras que cuando se preparan las preformas a partir de tejido seco, donde aún falta realizar la impregnación con la resina, se consideran procesos de vía seca.

En los procesos de vía húmeda, una vez colocado el prepreg, sólo es necesario realizar la compactación y el curado adecuado al tipo de preimpregnado. La compactación se puede realizar mediante presión en autoclave, con bolsa de vacío o mecánicamente. Los procesos con curado y compactación a alta presión en autoclave, son los más adecuados para piezas complejas de alta calidad, donde se necesita buen control sobre la impregnación y la uniformidad de la resina [22]. Hoy en día se han desarrollado nuevos tipos de prepregs que permiten realizar el curado fuera de autoclave (OoA), aspecto mucho más favorable desde el punto de vista medioambiental y de consumo de energía.

Los procesos por vía seca corresponden a los denominados Liquid Composite Molding (LCM), donde el tejido y la resina se mezclan una vez cerrado el molde y se puede observar una dirección clara de llenado o flujo de resina. Este proceso de llenado del molde con resina, se realiza con presión y/o vacío y, en algunos casos, con la posterior compactación de la pieza dentro del molde. 
Los procesos Liquid Composite Molding (LCM), se pueden clasificar en los procesos de inyección a presión positiva y por vacío (Figura 1.12). En los procesos de inyección a presión positiva destaca el RTM donde el molde es rígido, lo que permite un buen control dimensional de la pieza y un buen acabado en las dos caras de la pieza fabricada. En los procesos de vacío destaca el VARI, donde el semimolde inferior es rígido y el cierre se realiza mediante una bolsa de vacío.

Existen algunas variantes del proceso de RTM como son el RTM Light y el C-RTM. En el RTM Light el semimolde superior es flexible y el sellado del molde se realiza aplicando vacío perimetral. En el proceso C-RTM el molde se cierra en dos fases, en la primera fase el semimolde superior deja un gap por encima del tejido, donde se inyecta la resina, y en la segunda fase el semimolde superior desciende completamente forzando a la resina a empapar el tejido en la dirección del espesor, reduciendo así el tiempo de llenado frente al proceso de RTM convencional.

Los procesos LCM por vacío tienen el inconveniente de tener tiempos de llenado elevados, debido a la escasa diferencia de presiones entre la entrada y el frente de avance. Para reducir el tiempo de llenado, el ingeniero Seeman desarrolló el proceso Scrimp Seemann Composites Resin Infusion Molding (SCRIMP) en el año 1990. En esta variante se añade una malla de distribución que facilita el flujo en el proceso de llenado, de manera que la resina fluye en el plano de la pieza y a través de su espesor.

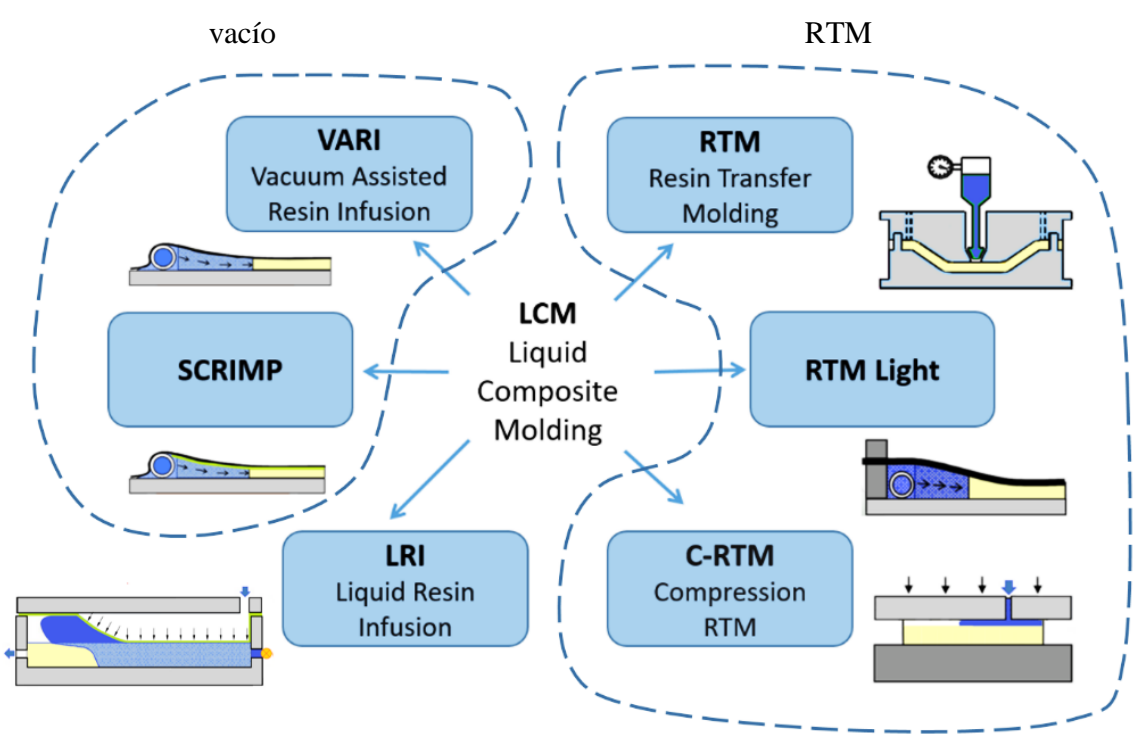

Figura 1.12. Procesos de conformado por vía seca. 
Por último, en el proceso Liquid Infusión (LRI), el sellado se realiza con una membrana flexible que se adapta perfectamente a la geometría de la pieza por efecto de la presión. Con este proceso se superan las limitaciones que ofrece el C-RTM para piezas que tienen geometría compleja.

\subsubsection{PROCESO RTM (RESIN TRANSFER MOLDING)}

El proceso RTM es un proceso en molde cerrado de vía seca, donde se inyecta la resina líquida a baja presión $(<4$ bar $)$. Se utilizan resinas de baja viscosidad $(<1 \mathrm{~Pa} \cdot \mathrm{s})$ que impregnan fácilmente el refuerzo de fibras colocado dentro del molde. Este proceso permite un buen control del refuerzo, así como la selección de la orientación de fibra, obteniendo buenas propiedades mecánicas con una alta tasa de refuerzo (del orden del 65\%). Se pueden fabricar piezas de gran complejidad, con buen acabado en ambas caras y precisión dimensional, permitiendo también la posibilidad de utilizar insertos.

El esquema del proceso se muestra en la Figura 1.13, donde se puede observar que en el caso del RTM la impregnación de la fibra con el material polimérico se lleva a cabo en el penúltimo paso, con el molde cerrado.
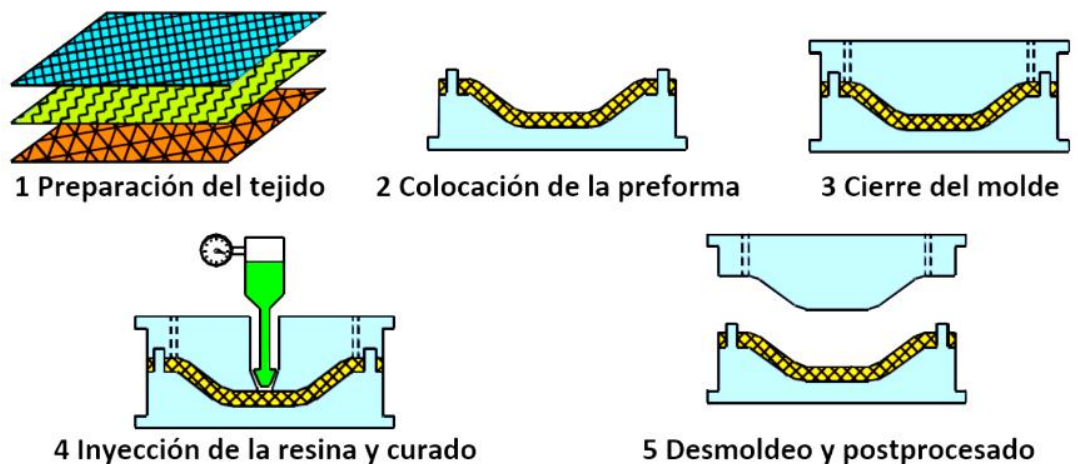

4 Inyección de la resina y curado

5 Desmoldeo y postprocesado

Figura 1.13. Esquema del Proceso RTM [23].

En el proceso de RTM es muy importante el control sobre cada fase del proceso para garantizar la calidad de la pieza fabricada. Las fases 1 y 2 de preparación y colocación del tejido son fundamentales para evitar problemas durante la fase de llenado y tener control sobre la orientación o la deformación del tejido. La fase 3 de cierre de molde debe garantizar el sellado, la correcta evacuación de aire y el flujo de la resina. Esta fase incluye la preparación del molde y de la resina. Tanto la preparación de la resina, como la fase de curado, se deben seguir las indicaciones precisas de la ficha del producto utilizado, para que la pieza alcance las propiedades adecuadas. En la fase 4 se lleva a cabo la inyección de la resina, con la impregnación del tejido y la 
polimerización de la resina. Por último, en la fase 5 se desmoldea y postprocesa la pieza, incluyendo también la limpieza y la preparación del molde.

\subsection{MECANIZADO DE MATERIALES COMPUESTOS}

Los procesos de fabricación de los materiales compuestos reforzados con fibra (RTM, infusión, etc.), permiten obtener piezas con una geometría cercana a la deseada. Tras el curado de estas piezas, se necesitan procesos de mecanizado para eliminar el material sobrante y conseguir las formas y tolerancias requeridas, así como para permitir los procesos de unión entre componentes. De entre los diferentes procesos de mecanizado, los más utilizados para este tipo de piezas son el taladrado y el fresado. Dentro del fresado, las operaciones más utilizadas son el ranurado y el contorneado (Figura 1.14), siendo esta última un recorte de los bordes del material o "edge trimming" [24-25].

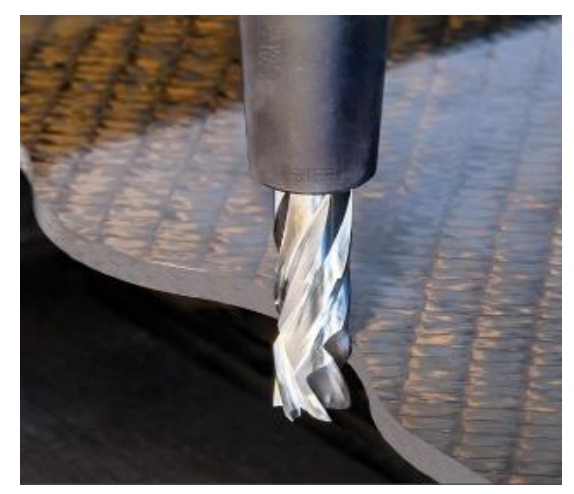

Figura 1.14. Contorneado o "edge trimming" [24].

Los materiales compuestos reforzados con fibra tienen un comportamiento diferente ante el mecanizado que los metálicos, debido a su naturaleza heterogénea y su anisotropía [26-27]. Los filos de corte de las herramientas generan la viruta por rotura del material compuesto, fracturando las fibras de refuerzo al mismo tiempo que se corta la matriz, y no por cizallamiento como ocurre en la mayoría de los metales. La maquinabilidad de un material compuesto dependerá mayoritariamente de las propiedades físicas y mecánicas de las fibras y la matriz, de la orientación de las fibras con respecto a la dirección del mecanizado y del porcentaje de fibra en el material [28-29].

La resistencia mecánica de las fibras abrasivas produce un desgaste rápido de la herramienta y un mal acabado superficial, pudiendo clasificar los distintos daños causados en el material durante el proceso de mecanizado, en tres tipos, [25, 30]: 
- Daños mecánicos: provocan delaminado y roturas en la superficie del material.

- Daños térmicos: se producen por una temperatura excesivamente elevada durante el mecanizado, provocando el quemado de la matriz y de las fibras.

- Daños químicos: se producen cuando el mecanizado se realiza con fluido de corte, provocando la destrucción de la unión entre las fibras y la matriz.

\subsubsection{HERRAMIENTAS DE MECANIZADO}

Las herramientas de corte para el mecanizado de materiales compuestos deben tener una buena resistencia al desgaste, reducir las fuerzas de corte generadas y las temperaturas durante el proceso, obteniendo la calidad y acabado de la pieza adecuado, con una buena productividad y eficiencia del proceso.

La productividad es un problema a la hora de seleccionar las herramientas de corte, porque la vida de la herramienta está limitada por el desgaste que producen las fibras abrasivas. Actualmente, existen una gran variedad de materiales de herramientas y geometrías disponibles. En relación al material de las herramientas empleadas para el mecanizado de materiales de fibra de carbono o de vidrio, las más utilizadas suelen ser de diamante [31], de metal duro, y metal duro recubierto de diamante, por las prestaciones que ofrecen.

En el fresado de materiales compuestos se suele trabajar con herramientas enterizas o de plaquitas (Figura 1.15), [32-33]. Dentro de las enterizas destacan las fresas helicoidales y los routers. En las fresas helicoidales, la hélice genera una fuerza axial que afecta al delaminado, por lo que se trabaja con fresas de ángulo de hélice reducido. Para evitar este defecto, también se pueden utilizar herramientas basadas en una doble hélice o estriadas, donde se oponen mutuamente los filos y comprimen del material.

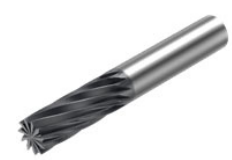

Ángulo de hélice reducido

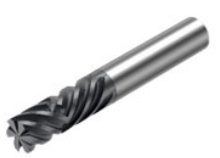

Doble hélice

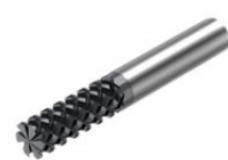

Estriada

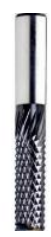

Router

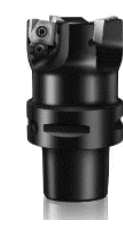

Fresa de planear de corte ligero

Figura 1.15. Herramientas de fresado para materiales compuestos [32-33].

Las herramientas pierden su capacidad de corte tras un cierto tiempo mecanizando, pudiendo definir el tiempo de vida o de duración de una 
herramienta como el periodo de tiempo en el que la herramienta puede ser utilizada para mecanizar, porque es capaz de conseguir las tolerancias y el acabado superficial requeridos en la pieza [34]. Este tiempo de vida está relacionado con el desgaste progresivo que sufre la herramienta. El tiempo de vida de la herramienta depende de varios factores, como son las condiciones de mecanizado (velocidad, avance y profundidad), la geometría de la herramienta, el porcentaje de fibra del material, etc.

En el mecanizado de materiales compuestos, los mecanismos básicos de desgaste que actúan sobre la herramienta son, principalmente la abrasión y las microfracturas, que progresivamente provocan pérdida de material en la herramienta. La abrasión se debe principalmente al efecto del roce de la herramienta con las fibras del material, mientras que las microfracturas son causadas por la oscilación de las fuerzas de corte que se producen cuando se fracturan las fibras del material. En el mecanizado de materiales compuestos, la temperatura no alcanza valores tan elevados como en el caso de los metales [30], por lo que el desgaste de la herramienta por mecanismos químicos es menor que el desgaste por mecanismos mecánicos.

El desgaste de la herramienta se observa principalmente en un redondeo del filo de corte y en las caras de incidencia y desprendimiento (Figura 1.16). En la cara de incidencia se genera una banda paralela a la arista de corte, que puede no ser uniforme, y cuya profundidad se evalúa mediante el parámetro denominado desgaste de flanco $(\mathrm{Vb})$. Uno de los criterios más utilizados para determinar la vida de la herramienta es la evolución del parámetro $\mathrm{Vb}$ con el tiempo, procediendo a cambiar la herramienta cuando se alcancen unos valores predeterminados de este parámetro, ya que el valor de este parámetro influye en las tolerancias y en el acabado superficial obtenido en la pieza.
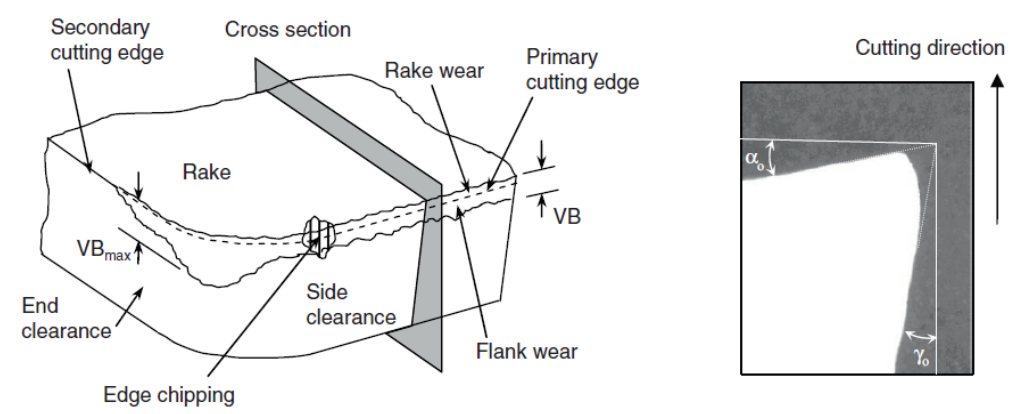

Figura 1.16. Zonas de desgaste de la herramienta [29]. 


\subsection{ESTADO DEL ARTE DEL FRESADO DE MATERIALES COMPUESTOS}

Las piezas fabricadas con materiales compuestos de matriz polimérica reforzados con fibras han sido objeto de estudio por la comunidad científica en las últimas décadas debido, sobre todo, a sus buenas prestaciones en los sectores de la automoción, el aeroespacial, el náutico, etc. Dado que, tras el curado de las piezas, son necesarios procesos de mecanizado para obtener las geometrías y dimensiones requeridas, existen numerosos estudios que abordan el mecanizado de este tipo de piezas.

Los procesos de mecanizado convencionales estudiados en la literatura son el torneado [35-37], el taladrado [38-46] y el fresado [47-53] entre otros, predominando estos dos últimos, ya que es usual el mecanizado de agujeros para el posterior ensamblaje entre piezas con uniones mecánicas, y el contorneado o recorte de los bordes mediante el proceso de fresado para la obtención de la geometría y las dimensiones finales de la pieza.

El principal problema encontrado cuando se analiza el estado del arte es la diversidad de estudios de mecanizado realizados por la comunidad científica, siendo difícil comparar entre ellos, debido a la combinación de parámetros y variables tratadas [54]. La mayoría de ellos realizan diferentes pruebas modificando los valores de unas variables, llamadas de entrada $o$ experimentales, de manera que sea posible observar e identificar su influencia y cambios en otras variables, llamadas de salida.

Las variables de entrada se centran, principalmente, en el estudio de las condiciones de corte (velocidad de corte, avance y profundidad), las características de las herramientas (geometría y material) y las características del material compuesto (el volumen de fibra y la orientación de la fibra).

Las variables de salida, se centran en la evaluación de la calidad de la pieza y en el análisis del proceso, pudiendo identificar entre ellas el acabado superficial de la pieza, las fuerzas generadas durante el corte, la potencia consumida, el desgaste de la herramienta, el delaminado, la temperatura alcanzada durante el proceso de corte, etc.

El tratamiento de las variables de entrada y salida, para ver la influencia de las primeras sobre las segundas, se ha realizado de diferentes formas en la literatura. En bastantes artículos se ha optado por un Diseño de Experimentos (DOE), poniendo las variables de entrada a diferentes niveles (usualmente dos o tres). Con los resultados obtenidos, se ha realizado un análisis de varianzas (ANOVA), comparando las variables de salida, con los diferentes niveles de las variables de entrada. 
Algunos artículos también plantean modelos de predicción de las variables de salida, como el degaste de las herramientas, el acabado superficial, las fuerzas generadas o el delaminado, utilizando modelos de regresión o técnicas de optimización como las redes neuronales o los algoritmos genéticos. Estos algoritmos consiguen resultados aceptables para tejidos con orientación de fibra unidimensional, pero se observan desviaciones en el caso de tejidos multidireccionales [55].

Atendiendo a la situación actual, los estudios realizados hasta el momento por la comunidad científica y a los objetivos de esta tesis, el estado del arte se va a clasificar en los siguientes puntos, resaltando las principales aportaciones en cada uno de ellos.

- Materiales, fabricación de laminados y operaciones de fresado.

- Herramientas de mecanizado.

- Estudio de la rugosidad superficial.

- Estudio del desgaste de la herramienta.

- Estudio del delaminado.

- Técnicas de optimización aplicadas.

\subsubsection{MATERIALES, FABRICACIÓN DE LAMINADOS Y OPERACIONES DE FRESADO}

La mayoría de estudios de la literatura se centran en los materiales poliméricos reforzados con fibras de carbono (CFRP) y de vidrio (GFRP), por ser los más utilizados en el sector industrial, no encontrándose casi ningún estudio que aborde el fresado de los materiales reforzados con fibra de basalto (BFRP).

Por otro lado, los laminados de materiales compuestos estudiados presentan diferente tipología (Tabla 1.4), encontrando principalmente tejidos unidireccionales con diferentes orientaciones de fibras en las capas del refuerzo, y tejidos bidireccionales.

La fabricación y características de los materiales compuestos de partida, para su posterior fresado, presenta diferencias entre los diferentes autores. El proceso de fabricación del composite tiene una fuerte influencia sobre los defectos que se producen más tarde en el mecanizado de la pieza [55]. Los laminados han sido fabricados con diferentes técnicas, como el moldeo por transferencia de resina (RTM) $[47,56]$, el sheet compression molding (SMC) 
$[51,57]$, los procesos de fabricación a partir de prepregs [58-60] y procesos manuales capa a capa [61-63].

El contenido o porcentaje de fibra presente en el laminado es otro parámetro importante, que hace al material más o menos abrasivo, y que también varía entre los autores. Es posible encontrar laminados con un bajo porcentaje de fibra, del orden del 33\% de fibra [64] y laminados que tienen un elevado porcentaje, hasta un $81 \%$ de fibra [57]. Además, los laminados presentan un número variable de capas de refuerzo, desde 8 en Li M. et al. [56] hasta 43 en He Y. et al. [49], y una dimensión variable del espesor a mecanizar.

Tabla 1.4. Compuestos laminados para fresado.

\begin{tabular}{|c|c|c|}
\hline Material & Lámina & Referencias \\
\hline \multirow[t]{2}{*}{ CFRP } & Unidireccional & $\begin{array}{l}\text { Slamani M. et al. [65]; Rimpault X. et al. [66]; Voss } \\
\text { R. et al. [67]; Liu G. et al. [68]; Robbany F. et al. } \\
\text { [69]; He Y. et al. [49]; Wang F. et al.2017 [70]; } \\
\text { Wang D. et al. [71]; Hintze W. } 2011 \text { [72]; Sundi S.A. } \\
2020 \text { [73] }\end{array}$ \\
\hline & Tejido & $\begin{array}{l}\text { Sheikh-Ahmad, et al. } 2017 \text { [74]; Davim, J.P. et al. } \\
2005 \text { [48]; Li, M. et al. [56]; Masek, P. et al. [53]; } \\
\text { Hintze, W. et al. } 2015 \text { [75]; Ozkan D. [60] }\end{array}$ \\
\hline \multirow[t]{2}{*}{ GFRP } & Unidireccional & $\begin{array}{l}\text { Azmi. A.I. et al. [47]; Jenarthanan M.P. et al. [63]; } \\
\text { Praveen P. et al. [61]; Neeli N. et al. [76] }\end{array}$ \\
\hline & Tejido & Vasudevan H. et al. [58]; Erkan Ö et al. [57] \\
\hline \multirow[t]{2}{*}{ BFRP } & Unidireccional & --- \\
\hline & Tejido & Navarro-Mas M.D. et al. [77-78] \\
\hline
\end{tabular}

Los trabajos de la literatura se van a clasificar en función de la operación de fresado que estudian: ranurado o contorneado (Tabla 1.5), siendo la de ranurado mucho más estudiada que la de contorneado.

Tabla 1.5. Operaciones de fresado.

\begin{tabular}{cl}
\hline Operación & \multicolumn{1}{c}{ Referencias } \\
\hline & Jenarthanan M.P. et al. [63]; Praveen P. et al. [61]; He Y. et al.[49]; \\
& Vasudevan H. et al. [58]; Sreenivasulu R.et al. [64]; Erkan Ö. et al. [57]; \\
& Davim J.P. et al. 2005 [48]; Razfar M.R. et al. [62]; Voss R. et al. [67]; \\
Ranurado & Jenarthanan M.P. et al. [50]; Li M. et al. [56]; Liu G. et al. [68]; \\
& Robbany F. et al. [69]; Neeli N. et al. [76]; Kilickap E. et al. [51]; \\
& Hintze W. et al. [2011,2015,2017,2013,2018]; Geis T. et al. [79]; \\
& Wang D. et al. [71]; Islam F. et al. [80] \\
\hline
\end{tabular}




\begin{tabular}{cl}
\hline Operación & \multicolumn{1}{c}{ Referencias } \\
\hline & Sheikh-Ahmad J. et al. [29]; Li N. et al. [52]; Masek P. et al. [53]; \\
Contorneado & Wang F. et al. [81]; Navarro-Mas et al. [77-78]; Rimpault X, et al. [66]; \\
& $\begin{array}{l}\text { Nguyen-Dinh N. et al. [82]; Ozkan D. et al. 2020 [60]; Sundi S. et al. } \\
\text { [73]; Slamani M. et el. [65] }\end{array}$ \\
\hline
\end{tabular}

\subsubsection{HERRAMIENTAS DE MECANIZADO}

Las herramientas utilizadas para el mecanizado de los laminados presentan diferentes materiales, diámetros y geometrías. Estos parámetros según su elección, permiten mejorar su eficacia en ciertos aspectos mientras que la reducen en otros, [83]. En relación a los materiales de las herramientas, la mayoría de autores utiliza los siguientes:

- Carburo cementado sin recubrimientos (Carburo).

- Carburo cementado con recubrimiento de diamante mediante deposición química (Carburo recubierto).

- Diamante policristalino (PCD).

El diámetro de la fresa utilizada (Ø), así como el número de filos (z) es variable, aunque se aprecia una tendencia a utilizar fresas de pequeño diámetro. Esto es debido a que en muchos de los trabajos se mecanizan ranuras, por lo que un tamaño de fresa pequeño es adecuado. En los estudios de contorneado también se utilizan estas fresas con diámetros pequeños, no existiendo casi estudios con fresas de diámetros superiores a $15 \mathrm{~mm}$.

La mayoría de las herramientas utilizadas son fresas enterizas (E), no existiendo casi trabajos con fresas de plaquitas (P). Algunos autores utilizan herramientas tipo router $(\mathrm{R})$, para eliminar el delaminado que produce el ángulo de hélice de la herramienta.

En relación a la geometría de las herramientas, éstas presentan diferentes ángulos de incidencia $(\alpha)$, desprendimiento $(\gamma)$ y de hélice $(\mathrm{L})$. La Tabla 1.6 muestra una comparativa entre diferentes autores, con las herramientas utilizadas para fresar el laminado.

Tabla 1.6. Características de las herramientas utilizadas.

\begin{tabular}{|c|c|c|c|c|c|c|c|}
\hline \multirow{2}{*}{ Referencias } & \multicolumn{3}{|c|}{ Tipo Herramienta } & \multirow[t]{2}{*}{ Material } & \multirow{2}{*}{$\begin{array}{c}\varnothing \\
(\mathrm{mm})\end{array}$} & \multirow[t]{2}{*}{ Filos } & \multirow[t]{2}{*}{ Geometría } \\
\hline & $\mathrm{R}$ & $\mathrm{E}$ & $\mathrm{P}$ & & & & \\
\hline $\begin{array}{l}\text { Sheikh- } \\
\text { Ahmad J. [74] }\end{array}$ & $\mathrm{X}$ & & & Carburo & 6.25 & & $\mathrm{~L} 60^{\circ} /-65^{\circ}$ \\
\hline
\end{tabular}


CAPÍTULO 1. PANORAMA CIENTÍFICO

\begin{tabular}{|c|c|c|c|c|c|c|c|}
\hline \multirow{2}{*}{ Referencias } & \multicolumn{3}{|c|}{ Tipo Herramienta } & \multirow[t]{2}{*}{ Material } & \multirow{2}{*}{$\begin{array}{c}\varnothing \\
(\mathrm{mm})\end{array}$} & \multirow[t]{2}{*}{ Filos } & \multirow[t]{2}{*}{ Geometría } \\
\hline & $\mathrm{R}$ & $E$ & $\mathrm{P}$ & & & & \\
\hline $\begin{array}{l}\text { Azmi A.I. et } \\
\text { al. [47] }\end{array}$ & & $X$ & & Carburo & 12 & 4 & a 9-16 $; 6^{\circ}$ L30 \\
\hline $\begin{array}{l}\text { Jenarthanan } \\
\text { M.P. [63] } \\
\end{array}$ & & $X$ & & PCD & 3 & & \\
\hline $\begin{array}{l}\text { Praveen P. et } \\
\text { al. [61] }\end{array}$ & & $X$ & & $\begin{array}{l}\text { Carburo/ } \\
\text { Carb recubierto }\end{array}$ & 10 & 4 & \\
\hline $\begin{array}{l}\text { Vasudevan H. } \\
\text { et al. [58] }\end{array}$ & & $X$ & & $\mathrm{PCD}$ & 10 & 2 & \\
\hline $\begin{array}{l}\text { Sreenivasulu } \\
\text { R. et al. [64] }\end{array}$ & & $\mathrm{X}$ & & Carburo & 10 & 4 & \\
\hline $\begin{array}{l}\text { Erkan Ö. et al. } \\
\text { [57] }\end{array}$ & & $X$ & & Carburo & 8 & $2-3-4$ & $\gamma 10^{\circ} ; \mathrm{L} 30^{\circ}$ \\
\hline $\begin{array}{l}\text { Davim J.P. et } \\
\text { al. [48] }\end{array}$ & & $\mathrm{X}$ & & Carburo & 6 & $2-6$ & $\alpha 9^{\circ} ; \gamma 10^{\circ} ; \mathrm{L} 30^{\circ}$ \\
\hline $\begin{array}{l}\text { Razfar M.R. et } \\
\text { al. [62] }\end{array}$ & & $X$ & & HSS & 4 & $2-4$ & \\
\hline $\begin{array}{l}\text { Slamani M. et } \\
\text { al. [84] }\end{array}$ & & $\mathrm{X}$ & & $\begin{array}{l}\text { Carburo } \\
\text { recubierto }\end{array}$ & 9.52 & 6 & $\alpha 10^{\circ} ; \gamma 8^{\circ} ; \mathrm{L} 10^{\circ}$ \\
\hline $\begin{array}{l}\text { Rimpault X. et } \\
\text { al. [66] }\end{array}$ & & $\mathrm{X}$ & & $\begin{array}{l}\text { Carburo } \\
\text { recubierto }\end{array}$ & 9.52 & 6 & $\alpha 10^{\circ} ; \gamma 8^{\circ} ; \mathrm{L} 10^{\circ}$ \\
\hline $\begin{array}{l}\text { Voss R. et al. } \\
\text { [67] }\end{array}$ & & & $\mathrm{X}$ & Carburo & 25 & 1 & $\begin{array}{l}\alpha 14^{\circ}, 21^{\circ} \\
\gamma 1^{\circ}, 15^{\circ}, 20^{\circ}\end{array}$ \\
\hline $\begin{array}{l}\text { Jenarthanan } \\
\text { M.P. [50] }\end{array}$ & & $\mathrm{X}$ & & Carburo & 5 & & $\mathrm{~L}^{2} 5^{\circ}, 35^{\circ}, 45^{\circ}$ \\
\hline $\begin{array}{l}\text { Li M.et al. } \\
{[56]}\end{array}$ & & $X$ & & Carburo & 6.35 & 4 & $\mathrm{~L}^{3} 5^{\circ}$ \\
\hline $\begin{array}{l}\text { Liu G. et al. } \\
\text { [68] }\end{array}$ & & $\mathrm{X}$ & & PCD & 12 & & $\alpha 10^{\circ} ; \gamma 3^{\circ} ; \mathrm{L}^{\mathrm{o}}$ \\
\hline $\begin{array}{l}\text { Slamani M. et } \\
\text { al. [65] }\end{array}$ & & $\mathrm{X}$ & & $\begin{array}{l}\text { Carburo } \\
\text { recubierto }\end{array}$ & 9.52 & 6 & $\alpha 8^{\circ} ; \gamma 10^{\circ} ; \mathrm{L} 10^{\circ}$ \\
\hline $\begin{array}{l}\text { Robbany F. et } \\
\text { al. [69] }\end{array}$ & & $\mathrm{X}$ & & Carburo & 6 & 4 & \\
\hline $\begin{array}{l}\text { Neeli N. et al. } \\
\text { [76] }\end{array}$ & & $\mathrm{X}$ & & Carburo & 5 & & $\mathrm{~L} 25^{\circ}, 35^{\circ}, 45^{\circ}$ \\
\hline $\begin{array}{l}\text { Kiliçkap E. et } \\
\text { al. [51] }\end{array}$ & & $\mathrm{X}$ & & Carburo & 10 & $3-4$ & $\mathrm{~L} 30^{\circ}$ \\
\hline $\begin{array}{l}\text { He Y. et al. } \\
\text { [49] }\end{array}$ & & $\mathrm{X}$ & & Carburo & 8 & 2 & $\alpha 10^{\circ} ; \gamma 6^{\circ} ; \mathrm{L} 0^{\circ}$ \\
\hline $\begin{array}{l}\text { Masek P. et al. } \\
{[53]}\end{array}$ & & $\mathrm{X}$ & & Carburo & 12 & 2 & $\alpha 10^{\circ} ; \gamma 0^{\circ} ; \mathrm{L} 2.5^{\circ}$ \\
\hline $\begin{array}{l}\text { Azmi A.I. et } \\
\text { al. [85] }\end{array}$ & & $\mathrm{X}$ & & $\begin{array}{l}\text { PCD y } \\
\text { Carb recubierto }\end{array}$ & 12 & & $\mathrm{~L} 30^{\circ}$ \\
\hline $\begin{array}{l}\text { Hintze W. et } \\
\text { al. } 2015 \text { [75] }\end{array}$ & & $\mathrm{X}$ & & Carburo & 8 & 2 & $\begin{array}{l}\alpha 10^{\circ}, 12^{\circ}, 14^{\circ} ; \\
\gamma-2^{\circ}, 0^{\circ} 2^{\circ} ; L-2^{\circ}, 0^{\circ}, 2^{\circ}\end{array}$ \\
\hline $\begin{array}{l}\text { Ozkan D. et al. } \\
2020 \text { [60] }\end{array}$ & & & $X$ & $\begin{array}{l}\text { Carb recubierto } \\
\text { TiN }\end{array}$ & 25 & 1 & \\
\hline $\begin{array}{l}\text { Sundi S.A. et } \\
\text { al. [73] }\end{array}$ & $\mathrm{X}$ & & & Carburo & 6.35 & 10 & $\mathrm{~L} 30^{\circ} /-35^{\circ}$ \\
\hline
\end{tabular}




\subsubsection{ESTUDIO DE LA RUGOSIDAD SUPERFICIAL}

La rugosidad superficial del laminado se estudia para obtener información de su calidad tras el fresado. La rugosidad obtenida puede afectar a su resistencia a la fatiga, a la fricción, a la capacidad para distribuir y mantener el lubricante, etc. [62].

En el estudio de la rugosidad superficial de los laminados de material compuesto, la mayoría de los autores evalúan el parámetro Ra (desviación media aritmética del perfil) [47-48, 51, 56, 61, 63-64, 68 y 73], aunque otros parámetros, como Rz, Rt o Rv han sido también evaluados, aunque con menos frecuencia $[65,74]$.

La medida de la rugosidad superficial en los materiales compuestos es compleja, y puede estar afectada por la fractura de la fibra durante el mecanizado, las fibras que sobresalen del laminado y por la degradación de la matriz, lo que puede provocar datos erróneos a la hora de evaluar este defecto $[47,73]$.

En la evaluación de la rugosidad superficial, los factores más estudiados han sido las condiciones de corte (velocidad de corte, avance y profundidad), obteniendo diferentes conclusiones entre los autores. Casi todos los autores coinciden en afirmar que la rugosidad superficial aumenta conforme aumenta el avance y disminuye conforme aumenta la velocidad de corte, [47-48, 51, 63]. Sin embargo algún autor, Li N. et al. [56] afirma que en tejidos unidireccionales con orientación de fibra de $45^{\circ} / 135^{\circ}$ el parámetro Ra aumenta al aumentar la velocidad de corte.

En relación a la profundidad de pasada, esta variable no se considera relevante por la mayoría de los autores, aunque algunos de ellos consideran que su efecto es relevante en la rugosidad, considerándolo incluso el parámetro más significativo [61, 64]. Liu G. et al. [68] afirman que la rugosidad aumenta conforme aumenta la profundidad de pasada. En cambio, Praveen P. et al. [61] afirman lo contrario, qué con una herramienta de carburo cementado sin recubrimiento, la rugosidad disminuye conforme aumenta la profundidad de pasada.

Además de las condiciones de corte, se han evaluado otros parámetros que influyen en la rugosidad superficial obtenida, como la orientación de la fibra, el ángulo de hélice de la herramienta o el número de filos de la herramienta. De esta forma, Jenarthanan M.P: et al. [63] concluyen que el parámetro Ra aumenta conforme aumenta el ángulo de orientación de la fibra y el ángulo de hélice de la herramienta. En relación a los filos de la herramienta, Razfar M.R. et al. [62] han observado que el valor de Ra es menor si la herramienta tiene menos filos. 


\subsubsection{Modelos empíricos}

La influencia de las condiciones de corte en la rugosidad superficial del laminado se ha expresado mediante modelos empíricos, utilizando regresión lineal multivariable. En la Tabla 1.7 se muestran algunas de las relaciones propuestas para la rugosidad, en función de la velocidad, el avance, la profundidad de corte y la longitud mecanizada.

Tabla 1.7. Modelos empíricos para rugosidad superficial en función de las condiciones de corte y por tipo de herramienta.

\begin{tabular}{|c|c|c|c|}
\hline Referencias & & Herramienta / Modelo propuesto & $\begin{array}{l}\text { Parámetro } \\
\text { rugosidad }(\mu \mathrm{m})\end{array}$ \\
\hline \multirow[t]{2}{*}{$\begin{array}{l}\text { Sheikh-Ahmad } \\
\text { J. et al. [29] }\end{array}$} & 2012 & $\begin{array}{l}\text { Solid carbide router burr tool } \quad \varnothing=6.3 \mathrm{~mm} \\
R_{z}=7.8956+1.4745 v_{f}+0.1285 l_{c}-4.2344 \times 10^{-3} v_{c} v_{f}\end{array}$ & $7.5<\mathrm{Rz}<28$ \\
\hline & & $v_{c}(\mathrm{~m} / \mathrm{min}) ; v_{f}(\mathrm{~m} / \mathrm{min}) ; l_{c}(\mathrm{~m})$ & \\
\hline \multirow[t]{2}{*}{$\begin{array}{l}\text { Sheikh-Ahmad } \\
\text { J. et al. [74] }\end{array}$} & 2017 & 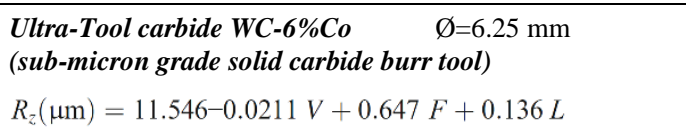 & - \\
\hline & & $V(\mathrm{~m} / \mathrm{min}) ; F(\mathrm{~m} / \mathrm{min}) ; L(\mathrm{~m})$ & \\
\hline \multirow{2}{*}{$\begin{array}{l}\text { Azmi A.I. et } \\
\text { al. [47] }\end{array}$} & 2013 & $\begin{array}{l}\text { Uncoated tungsten carbide K20 } \quad \emptyset=12 \mathrm{~mm} \\
R_{a}=10^{0.293} \times s^{-0.232} \times f^{0.313} \times d^{0.025}\end{array}$ & $\mathrm{Ra}<3$ \\
\hline & & $s(\mathrm{rpm}) ; f(\mathrm{~mm} / \mathrm{min}) ; d(\mathrm{~mm})$ & \\
\hline \multirow{4}{*}{$\begin{array}{l}\text { Praveen P. et } \\
\text { al.[61] }\end{array}$} & a) & $\begin{array}{l}\text { Ti-Namite coated carbide end mill } \quad \varnothing=10 \mathrm{~mm} \mathrm{z}=4 \\
\mathrm{Ra}=3.74+8.8 \times 10^{-3} \mathrm{~V}+8.7 \times 10^{-4} \mathrm{f}-7.3 \times 10^{-2} \mathrm{~d}\end{array}$ & \\
\hline & $2010 \mathrm{~b})$ & $\begin{array}{l}\text { Solid Carbide end mill: } \\
\mathrm{Ra}=10.7-8.87 \times 10^{-2} \mathrm{~V}+0.0006 .5 \times 10^{-4} \mathrm{f}-4.12 \times 10^{-3} \mathrm{~d}\end{array}$ & a,b) $3<\mathrm{Ra}<9$ \\
\hline & c) & $\begin{array}{l}\text { Tipped carbide end mill: } \\
\mathrm{Ra}=3.74+8.8 \times 10^{-3} \mathrm{~V}+8.7 \times 10^{-4} \mathrm{f}-7.3 \times 10^{-2} \mathrm{~d} \\
\text { la misma aproximación que en el caso a) parece un error }\end{array}$ & c) $3<\mathrm{Ra}<5$ \\
\hline & & $V(\mathrm{~m} / \mathrm{min}) ; f(\mathrm{~mm} / \mathrm{min}) ; d(\mathrm{~mm})$ & \\
\hline \multirow{3}{*}{$\begin{array}{l}\text { Davim J.P. et } \\
\text { al. [48] }\end{array}$} & a) & $\begin{array}{l}\text { Cemented carbide (K10) } \quad \varnothing 10 \mathrm{~mm} \quad \mathrm{z}=2 \\
\mathrm{Ra}=1.76-2.40 \times 10^{-2} V+1.71 \times 10^{-3} f,\end{array}$ & \\
\hline & $2005 \mathrm{~b})$ & $\begin{array}{l}\text { Cemented carbide }(\text { K10) } \quad \varnothing 10 \mathrm{~mm} \quad \mathrm{z}=6 \\
\mathrm{Ra}=1.76-7.70 \times 10^{-3} V+1.75 \times 10^{-3} f\end{array}$ & \\
\hline & & $V(\mathrm{~m} / \mathrm{min}) ; f(\mathrm{~mm} / \mathrm{min})$ & \\
\hline \multirow[t]{2}{*}{$\begin{array}{l}\text { Liu G. et al. } \\
{[68]}\end{array}$} & 2017 & $\begin{array}{l}\text { Polycrystalline diamond } \varnothing=12 \mathrm{~mm} \text { (no indica } \mathrm{z} \text {, parece } \\
\mathrm{z}=4 \text { ) } \\
R_{a}=10^{1.147} v^{-0.227} a_{\mathrm{e}}^{0.083} f_{z}^{0.236}\end{array}$ & $2<\mathrm{Ra}<5$ \\
\hline & & $v(\mathrm{~m} / \mathrm{min}) ; a_{e}(\mathrm{~mm}) ; f_{z}(\mathrm{~mm} /$ diente $)$ & \\
\hline
\end{tabular}




\begin{tabular}{|c|c|c|c|}
\hline Referencias & & Herramienta / Modelo propuesto & $\begin{array}{l}\text { Parámetro } \\
\text { rugosidad }(\mu \mathrm{m})\end{array}$ \\
\hline \multirow[t]{2}{*}{$\begin{array}{l}\text { Slamani M. et } \\
\text { al. [65] }\end{array}$} & 2019 & $\begin{array}{l}\text { CVD diamond-coated carbide } \quad \varnothing=9.525 \mathrm{~mm} \mathrm{z}=6 \\
\hat{R}_{a}=2.58-0.09 \times 10^{-3} \times f+8.72 \times 10^{-3} \times V_{C}+0.5 \times 10^{-5} \times f\end{array}$ & $5<\mathrm{Ra}<12$ \\
\hline & & $V c(\mathrm{~m} / \mathrm{min}) ; f(\mathrm{~mm} / \mathrm{rev})$ & \\
\hline
\end{tabular}

Los modelos propuestos son difícilmente comparables por dos motivos. El primer motivo es que las herramientas utilizadas en el mecanizado influyen directamente en el acabado superficial de la pieza. Dados los diferentes tipos de herramienta, diámetros, número de dientes y sus condiciones de corte, la huella sobre la superficie mecanizada y el valor del parámetro de rugosidad superficial evaluado no es comparable. El segundo motivo es el material mecanizado, ya que el tamaño de las mechas y las fibras individuales de tejido, así como la proporción de resina en función del volumen de fibra, afectan directamente a cómo se produce la rotura de la fibra y a la variación de los valores de rugosidad medidos.

Sin embargo, sí se pueden analizar las variables significativas que se identifican aplicando estos modelos de predicción y las tendencias observadas con el cambio de condiciones de mecanizado. De esta forma se puede observar, que el avance en todos los casos tiene una influencia positiva sobre el parámetro de rugosidad, es decir si aumenta el avance, aumenta el valor del parámetro de rugosidad. No ocurre así con la velocidad de corte que tiene una influencia a veces positiva y otras, negativa, y en algunos casos se afirma que no afecta. Respecto a la profundidad de corte y a la longitud mecanizada es analizada tan sólo en casos puntuales por lo que no se puede plantear ninguna predicción.

\subsubsection{ESTUDIO DEL DESGASTE DE LA HERRAMIENTA}

En el fresado de materiales compuestos reforzados con fibra, el estudio del desgaste de la herramienta se ha realizado mayoritariamente midiendo el desgaste producido en el flanco de la herramienta, denominado $\mathrm{Vb}$ [47, 8384]. Cuando este desgaste alcanza un determinado valor se concluye que la herramienta no puede seguir mecanizando. En ese instante se puede calcular el tiempo de vida de la herramienta y la longitud mecanizada, para unas determinadas condiciones de corte. Este valor de $\mathrm{Vb}$ se puede fijar en $0.3 \mathrm{~mm}$ para la operación de fresado, [47, 66] de acuerdo a la norma ISO 8688-2 [86]. En el estudio de Azmi A.I. [85], este valor de $\mathrm{Vb}$ de $0.3 \mathrm{~mm}$ de desgaste se alcanza para un tiempo de vida entre 10 y 12 minutos, dependiendo de las 
condiciones de corte. Azmi también indica la dificultad en la medida del desgaste sobre la cara de incidencia, debido al redondeo de la punta y la viruta adherida cuando se trabaja en ciertas condiciones [47].

Los factores más relevantes que se han tratado en el estudio del desgaste son las condiciones de corte (velocidad, avance y profundidad). Los diferentes autores coinciden en afirmar que el desgaste de la herramienta aumenta al aumentar la velocidad de corte [84]. En relación al avance, un avance más pequeño provoca un desgaste menor en la herramienta [67, 84].

Respecto a la profundidad de pasada que ha sido mucho menos estudiada, Kim M. [87] afirma que profundidades de pasada pequeñas por debajo de 1 $\mathrm{mm}$, aceleran el desgaste del flanco, debido al aumento de la distancia de fricción sobre la cara de incidencia.

Recientemente, algunos autores han estudiado el efecto sobre el desgaste de algunas características del material como la orientación de la fibra como Kim M. [87] y Nguyen N. [82]. Ambos comprueban que el mecanizado de materiales compuestos a se realiza por la fractura de las fibras y esto provoca dos tipos de desgaste: el desgaste de la arista o borde de la herramienta y principalmente desgaste en la cara de incidencia o de flanco. El desgaste del borde de la herramienta es provocado por la fuerza de impacto de la fibra cortada sobre la herramienta, porque se produce un efecto abrasivo de las fibras rotas que chocan sobre la arista de corte. El desgaste en la cara de incidencia o de flanco causado por la fuerza de fricción con la superficie mecanizada. Kim M. et al. [87], concluyen que el desgaste de la herramienta está fuertemente influenciado por la orientación de la fibra. En su trabajo, con una longitud mecanizada de 7 metros, el desgaste de flanco es mucho mayor con una orientación de fibra de $0^{\circ}$, esta orientación coincide con la dirección paralela entre la fibra y la cara de incidencia. El valor de desgaste con una orientación de fibra de $90^{\circ}$ se reduce mucho.

Nguyen N. et al. [82] en su trabajo indican que el desgaste de flanco es mayor cuando se trabaja con una orientación de fibra de $45^{\circ}$, mientras que una orientación de $90^{\circ}$ produce un mayor redondeo del borde de la herramienta, independientemente de la velocidad de corte.

\subsubsection{Modelos empíricos}

Hay pocos autores que hayan descrito mediante modelos empíricos la influencia de las condiciones de corte en el desgaste de la herramienta en fresado. En la Tabla 1.8 se muestran algunas de las relaciones propuestas para el desgaste de la herramienta (Tw) o tiempo de vida (TL), siendo "V" la velocidad, "f" el avance, "d" la profundidad de corte y "L" la longitud mecanizada. 
Tabla 1.8. Modelos empíricos para el desgaste de la herramienta.

\begin{tabular}{|c|c|c|}
\hline Referencias & & Modelo propuesto \\
\hline \multirow[t]{2}{*}{ Azmi A.I. et al. [47] } & 2013 & $T L=10^{11.398} \times s^{-0.911} \times f^{-1.922} \times d^{-0.201}$ \\
\hline & & $\mathrm{S}(\mathrm{rpm}) ; \mathrm{f}(\mathrm{mm} /$ minuto $) ; \mathrm{d}(\mathrm{mm})$ \\
\hline \multirow[t]{6}{*}{ Slamani M. et al [84] } & 2015 & $\widehat{T_{w}}=e^{-25.745}\left(V_{C}\right)^{1.47}(f)^{1.75}(L)^{0.68}$ \\
\hline & & $\mathrm{Vc}(\mathrm{m} / \mathrm{min}) ; \mathrm{f}(\mathrm{mm} / \mathrm{min}) ; \mathrm{L}(\mathrm{mm})$ \\
\hline & & $T_{w}=e^{-0.0079 V_{C}-0.00089 f+0.282 L+0.000002 V_{C} \times f-0.00057 f \times l}$ \\
\hline & & $\mathrm{Vc}(\mathrm{m} / \mathrm{min}) ; \mathrm{f}(\mathrm{mm} / \mathrm{min}) ; \mathrm{L}(\mathrm{mm})$ \\
\hline & & $\widehat{T_{w}}=e^{18.95}\left(V_{C}\right)^{-2.287}(f)^{-1.8557}(L)^{0.3899}(F)^{0.9865}$ \\
\hline & & $\mathrm{Tw}(\mathrm{mm}) ; \mathrm{Vc}(\mathrm{m} / \mathrm{min}) ; \mathrm{f}(\mathrm{mm} / \mathrm{min}) ; \mathrm{L}(\mathrm{mm}) ; \mathrm{F}(\mathrm{N})$ \\
\hline
\end{tabular}

\subsubsection{ESTUDIO DEL DELAMINADO}

Uno de los principales problemas que aparece cuando se mecanizan materiales compuestos reforzados con fibras es el delaminado. Este defecto afecta tanto a la precisión dimensional como a la posterior unión entre piezas, provocando que las piezas tengan que ser reprocesadas, con la pérdida de tiempo que esto conlleva, e incluso que sean desechadas [72]. Por ello, este defecto ha sido ampliamente estudiado en los procesos de taladrado [39-40, $43,45]$, etc. y de fresado [47, 51, 57, 88, etc.], tratando de cuantificarlo y evaluando las causas que provocan su aparición.

El delaminado consiste en una separación de las capas de tejido del material compuesto debido a la fuerza que ejerce la herramienta durante el proceso de mecanizado. En el contorneado de una pieza, el defecto de delaminado únicamente aparece en la capa superior e inferior de la pieza, cuando en esta capa superficial no hay suficiente resina que sujete las mechas de tejido. La fuerza de la herramienta durante el proceso de corte dobla las hebras de fibra en vez de cortarlas, y se produce un recorte no uniforme del borde de la pieza que se denomina delaminado.

El delaminado se puede clasificar en tres tipos: I, II y III [89]. El tipo I aparece cuando las fibras se doblan hacia dentro de la línea de mecanizado, provocando un daño superficial en la pieza. El tipo II aparece cuando existen fibras que sobresalen del borde mecanizado, mientras que el tipo III aparece cuando existen fibras paralelas al borde mecanizado. También puede aparecer simultáneamente delaminados del tipo I/II (Figura 1.17). 


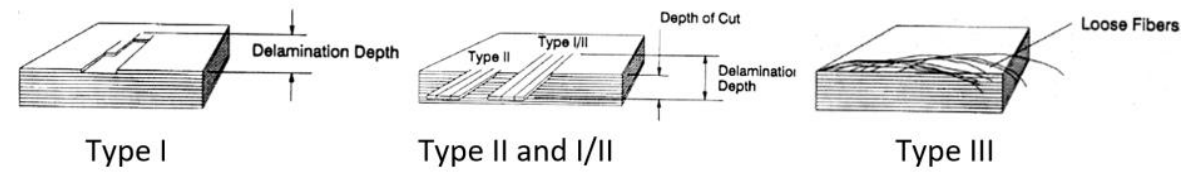

Figura 1.17. Tipos de delaminado [89].

\subsubsection{Medida del delaminado}

El delaminado producido en las piezas se ha cuantificado de diferentes formas. Antes de explicar cómo se hace en fresado, se verá cómo se ha evaluado en el taladrado por haberse estudiado con anterioridad al fresado, y así ver el paralelismo existente entre ambos procesos de mecanizado.

\subsection{Medida del delaminado en el taladrado}

En el taladrado, el delaminado aparece alrededor del agujero (Figura 1.18), tanto a la entrada como a la salida de la broca, $[41,46]$.

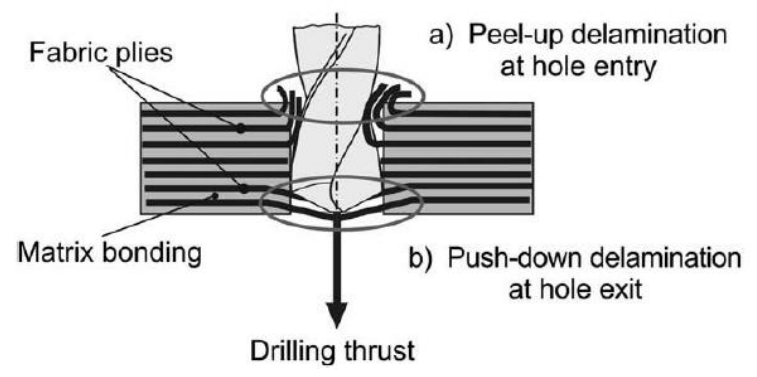

Figura 1.18. Delaminado en taladrado [41].

Para poder cuantificar este defecto se han definido diferentes factores de delaminado, más o menos complejos. Uno de los más sencillos es el "Factor de delaminado $\left(F_{d}\right)$ " (Figura 1.19) definido como el cociente entre el máximo diámetro del delaminado $\left(\mathrm{D}_{\max }\right)$ y el diámetro del agujero (D) (ecuación 1.1) [40]. 


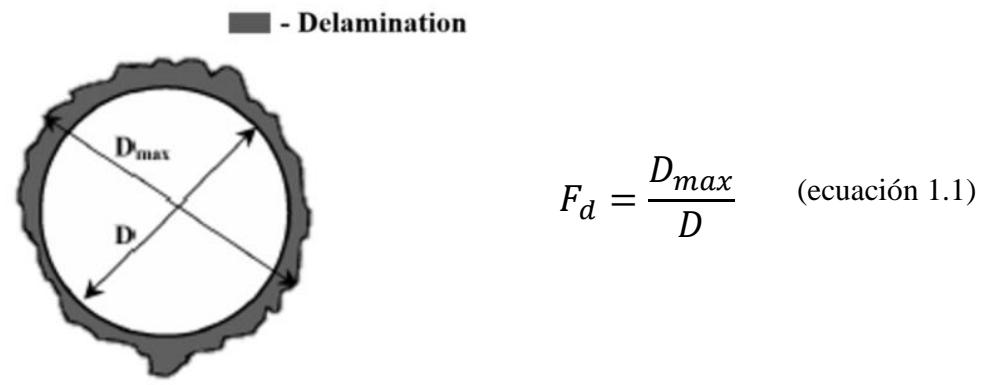

Figura 1.19. Factor de delaminado [40].

Este parámetro mide el delaminado unidimensionalmente, no reflejando el valor real del defecto (Figura 1.20), ya que proporciona el mismo valor para mecanizados con un delaminado continuo alrededor de todo el agujero o cuando sólo se presentan mechas finas y largas en algunos puntos alrededor del agujero [39].
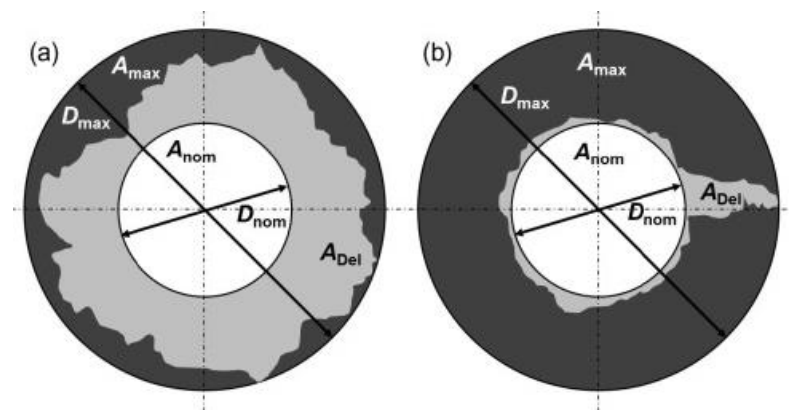

Figura 1.20. Posibles casos de evaluación del delaminado. (a) delaminación uniforme. (b) fibras largas con delaminación no uniforme [44].

Para evitar este inconveniente, se define un nuevo factor de delaminado, denominado "Factor de delaminado ajustado $\left(F_{d a}\right)$ " que evalúa la superficie del delaminado alrededor del agujero [44]. En este nuevo factor se añade un término adicional al factor de delaminado unidimensional, que tiene en cuenta las superficies invadidas por el delaminado. Los parámetros $\alpha$ y $\beta$ representan diferentes pesos para cada uno de los términos de la ecuación. Si el parámetro $\beta$ se define como la ratio entre el área de delaminado $\left(A_{d}\right)$ y la diferencia entre $A_{\max } \mathrm{y}_{0}$, el factor $\mathrm{F}_{\mathrm{da}}$ queda tal y como se muestra en la ecuación 1.2.

$$
\begin{gathered}
F_{d a}=\alpha \cdot \frac{D_{\max }}{D_{0}}+\beta \cdot \frac{A_{\max }}{A_{0}}=\alpha \cdot F_{d}+\beta \cdot F_{d}^{2}= \\
=F_{d}+\frac{A_{d}}{\left(A_{\max }-A_{0}\right)} \cdot\left(F_{d}^{2}-F_{d}\right)
\end{gathered}
$$


Diversos autores [43-45], evalúan estos parámetros de delaminado cuando se taladran materiales compuestos, tratando de ver la influencia que tienen diferentes variables en ellos. T. J. Grilo et al. [45] estudian el taladrado de CFRP, variando las condiciones de corte (velocidad de corte y avance) y trabajando con diferentes geometrías de herramientas, concluyendo que existe una relación directa entre los factores de delaminado y el avance. El factor de delaminado también es estudiado por Gaitonde V. N. et al. [43] en el taladrado de CFRP, variando la velocidad de corte, el avance y el ángulo de punta de la herramienta. Como conclusión destacan que el delaminado a la entrada del agujero se puede reducir aumentando la velocidad o trabajando con avances bajos y radios de punta de la herramienta adecuados.

\subsection{Medida del delaminado en el fresado}

En fresado, el delaminado se ha estudiado, principalmente en las operaciones de ranurado y contorneado. Para la medida del delaminado en ranurado, se define un "Factor de delaminado $\left(W_{d}\right)$ " similar al utilizado en taladrado (ecuación 1.3), solo qué en vez de referenciar la medida al diámetro del agujero, se referencia al ancho de la ranura, [48, 63].

Al igual que sucede en taladrado, este parámetro de medida es unidimensional y sólo tiene en cuenta el delaminado tipo I, que produce daño en la pieza. Este parámetro no evalúa los otros tipos de delaminado (II y III), que sobresalen dentro de la ranura, los cuales pueden ser más importantes que los del tipo I (Figura 1.21).

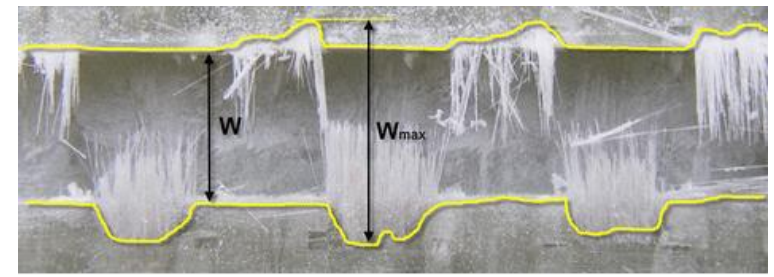

$$
\begin{aligned}
& W_{d}=\frac{W_{\max }}{W} \\
& \text { (ecuación 1.3) }
\end{aligned}
$$

Figura 1.21. Factor de delaminado en el ranurado [57].

En otros trabajos que evalúan el delaminado en fresado, se identifican los diferentes tipos de delaminado (tipos I, II y III), intentando determinar la importancia de cada uno de ellos y cuantificando su valor. En la operación de ranurado, aparecen trabajos como el de Li M. et al. [56] que evalúan los tres tipos de delaminado midiendo la longitud de las fibras que provocan cada uno de ellos. En trabajos similares, Sheikh-Ahmad J. [29, 74], evalúa el delaminado en la operación de contorneado midiendo, para cada tipo de delaminado, la longitud de las fibras y su frecuencia de aparición, y obteniendo la media de los valores. Estos trabajos concluyen, como primera aproximación, que el 
delaminado varía con las condiciones de corte (velocidad y avance) y con el desgaste de la herramienta.

Además de medir la longitud de las fibras que provocan los diferentes tipos de delaminado, se han evaluado también las superficies ocupadas por dichas fibras. De esta forma, en el mecanizado de madera, hay estudios [90] que miden el delaminado mediante un factor de delaminado denominado $A_{\text {del }}$, que se define como la relación entre el área de delaminado $\left(S_{\text {del }}\right.$ en $\left.\mathrm{mm}^{2}\right)$ y la longitud evaluada $\left(\mathrm{L}_{\mathrm{p}}\right)$ (ecuación 1.4). Con este factor se evalúan las áreas del delaminado, y no sólo las longitudes de las fibras deshilachadas. Este factor puede ser también aplicado a los materiales compuestos de matriz polimérica reforzados con fibra.

$$
A_{d e l}=\frac{S_{d e l}}{L_{p}} \quad \text { (ecuación 1.4) }
$$

Wang F. et al. [81], definen un factor de delaminado $F_{d-\text {-area }}$ como la relación entre el área delaminada $(A)$ y el área total mecanizada, representada por el producto de la profundidad de corte $\left(\mathrm{a}_{\mathrm{e}}\right)$ y la longitud de corte $\left(\mathrm{l}_{\text {cutting }}\right)$, (ecuación 1.5). Sus resultados están enfocados más al efecto de la geometría de la herramienta que al análisis de las condiciones de mecanizado.

$$
F_{d-\text { area }}=\frac{A}{a_{e} \cdot l_{\text {cutting }}} \quad \text { (ecuación 1.5) }
$$

Generalmente, el delaminado se produce mayoritariamente en una de las capas del material, la capa superior o la inferior, por lo que los factores definidos anteriormente sólo evalúan este efecto en una de las capas. Sin embargo, también se han definido factores de delaminado para intentar cuantificar conjuntamente este defecto en ambas capas [53]. En esta propuesta, se miden las áreas de delaminado en ambas capas $\left(\mathrm{A}_{\text {dtop }} \mathrm{y} \mathrm{A}_{\mathrm{dbottom}}\right)$ en la longitud mecanizada (1), tomando imágenes desde la parte superior, la inferior y la frontal de la pieza (ecuación 1.6).

$$
A_{d l}=\frac{A_{d t o p}+A_{d b o t t o m}}{l} \quad \text { (ecuación 1.6) }
$$

\subsubsection{Parámetros que influyen en el delaminado}

En la literatura, los parámetros más estudiados para evaluar el delaminado tras el fresado de la pieza son los siguientes: 
- Condiciones de corte (velocidad, avance y profundidad). Todos los autores que estudian las condiciones de corte incluyen la velocidad y el avance. Sólo algunos autores incluyen además la profundidad (radial y/o axial), ya que la mayoría de la comunidad científica opina que su influencia en el delaminado no es tan relevante como la velocidad y el avance.

- Orientación de las fibras. Se evalúa la orientación de las fibras con respecto a la dirección de mecanizado, con el objetivo de determinar las orientaciones que favorecen el delaminado.

- Herramienta. Se evalúa tanto el material de la herramienta, como diferentes parámetros de geometría, como el ángulo de incidencia, el de desprendimiento y el de hélice, el radio de punta y el número de filos de la herramienta.

Entre ellos, los más estudiados son las condiciones de corte y la orientación de las fibras. Los parámetros relacionados con la herramienta tienen mucha menos influencia en el delaminado y han sido menos estudiados.

Además, en el estudio del mecanizado de materiales reforzados con fibra se ha definido un nuevo parámetro que mide la distancia desde el borde mecanizado de la pieza hasta el cruce de la primera mecha de tejido (Xd). Este parámetro ha sido definido por Hintze W. [75], e influye en el delaminado del borde de la pieza.

\subsection{Condiciones de corte}

En el estudio de la influencia de las condiciones de corte en el delaminado, se va a distinguir entre las operaciones de ranurado y contorneado. Esta distinción se hace porque en los trabajos que abordan el ranurado, la delaminación se suele evaluar atendiendo al factor de delaminado definido como $F_{d}=\left(F_{\max } / F\right)$, mientras que, en los trabajos de contorneado, se suelen utilizar parámetros de medida diferentes para su evaluación.

\section{Ranurado}

En el ranurado, la influencia de las condiciones de corte en el delaminado $\left(F_{d}\right)$ no presenta consenso en los diferentes trabajos de la literatura. Hay autores que consideran que el avance tiene mayor influencia en el delaminado que la velocidad de corte $[50,57-58,62-63,76]$. Otros, en cambio, consideran más relevante la velocidad de corte que el avance [48-49, 64]. Finalmente, hay autores que afirman que la profundidad de pasada es el factor más influyente en el delaminado, por delante de la velocidad y el avance [61]. En general, un aumento en el avance y la velocidad provoca un aumento en el factor de 
delaminado, aunque también hay trabajos que afirman lo contrario, por ejemplo, que un aumento en la velocidad disminuye dicho factor.

Como se ha comentado anteriormente, los autores trabajan con herramientas de material y geometría diferente: diámetro, número de dientes, etc. Por ello, las condiciones de corte estudiadas para evaluar el delaminado, varían entre ellos. A continuación, se muestra de forma gráfica la influencia de las condiciones de corte en el factor de delaminado en diferentes trabajos de la literatura (Figura 1.22).
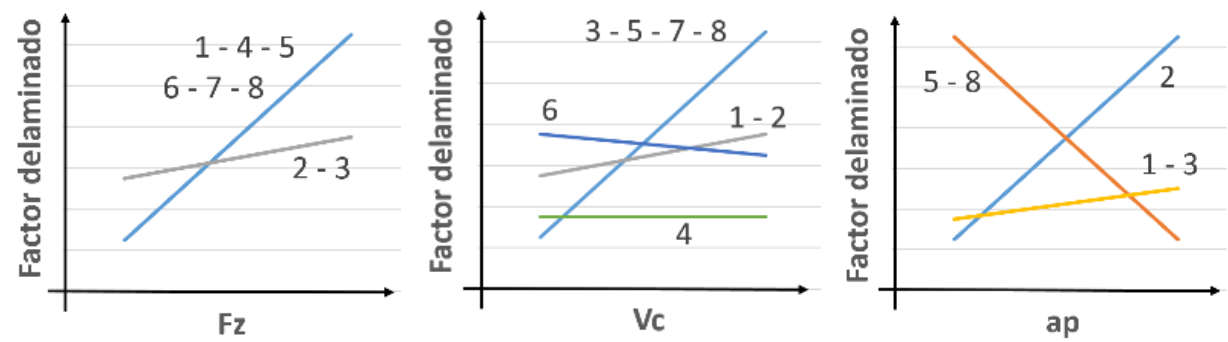

1 [63] Jenarthanan M.P. et al.

5 [62] Razfar M.R. et al.

2 [61] Praveen P. et al.

6 [50] Jenarthanan M.P. et al.

3 [64] Sreenivasulu R. et al.

7 [51]Kiliçkap E. et al

4 [48] Davim J.P. et al.

8 [57] Erkan Ö.et al.

Figura 1.22. Influencia de las condiciones de corte en el ranurado.

\section{Contorneado}

En la literatura hay pocos trabajos que estudien la medida del delaminado en el contorneado. Seguidamente se comentarán los trabajos de Sheikh-Ahmad J. et al. [74], Masek P. et al. [53], Wang F. et al. [81, 91] y el de Ozkan D. et al. [60] (Figura 1.23).

En el trabajo de Sheikh-Ahmad J. et al. [74] se destaca la naturaleza aleatoria de la delaminación. El delaminado se evalúa mediante un factor obtenido como la media de la longitud de las fibras deshilachadas. Este factor aumenta cuando aumenta el avance y decrece cuando aumenta la velocidad, siendo el avance el factor que más afecta al delaminado.

En el trabajo de Masek P. et al. [53] se evalúa el delaminado midiendo las áreas afectadas, concluyendo que el área delaminada decrece cuando aumentan la profundidad axial, el avance y la velocidad de corte, y decrece cuando 
aumenta la profundidad radial. Wang, F. et al. [81], evalúan el delaminado midiendo las áreas afectadas y comparando con el área mecanizada. El mecanizado se realiza con tres herramientas de corte multifilo con muescas de diferentes geometrías. Se trabaja con una profundidad de pasada constante y en su estudio se observa que el área delaminada decrece cuando aumentan la velocidad de corte y decrece el avance.

Por último, en el trabajo de Ozkan D. et al. [60] se determina que el avance es uno de los parámetros más influyentes, sin embargo, la profundidad del delaminado varía de forma diferente en distintos rangos de avance. En este estudio se observa un mayor deterioro de la pieza cuando se trabaja con avances altos $(0.25 \mathrm{~mm} /$ diente $)$ y velocidades de corte elevadas $(300 \mathrm{~m} / \mathrm{min})$.
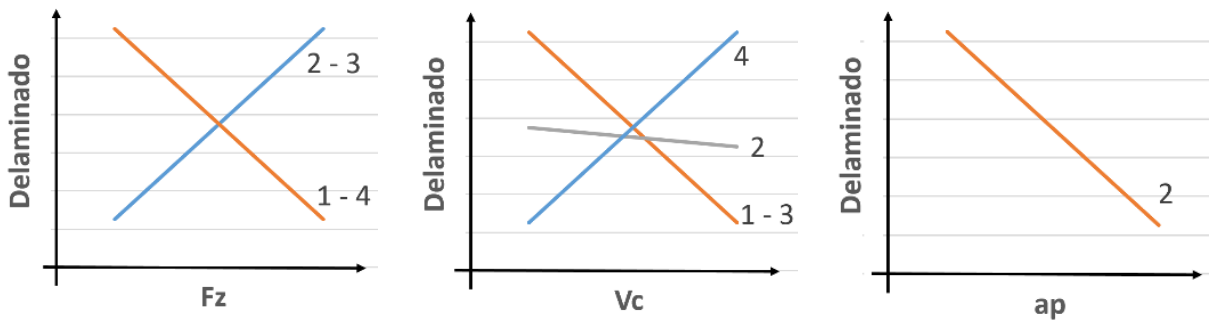

Factor analizado

1 [74] Sheikh-Ahmad J. et al.

2 [53] Masek P. et al.

3 [81] Wang F.

4 [60] Ozkan D.
Longitud media delaminada

Suma areas delaminadas: inferior y superior

Area delaminada frente a area mecanizada

Profundidad delaminada

Figura 1.23. Influencia de las condiciones de corte en el contorneado.

\section{Modelos empíricos}

La influencia de las condiciones de corte en el delaminado se ha descrito mediante modelos empíricos, utilizando regresión lineal multivariable. En la Tabla 1.9 se muestran algunas de las relaciones propuestas para el delaminado, siendo "V" la velocidad, "f" el avance, "d" la profundidad de corte y "L" la longitud mecanizada. 
Tabla 1.9. Modelos del delaminado en función de las condiciones de corte.

\begin{tabular}{|c|c|c|c|}
\hline Referencias & \multicolumn{2}{|l|}{ Modelo propuesto } & Herramienta \\
\hline \multirow{5}{*}{$\begin{array}{l}\text { Jenarthanan } \\
\text { M.P: et al. } \\
\text { [63] } \\
\text { (ranurado) }\end{array}$} & \multirow{4}{*}{\multicolumn{3}{|c|}{$\begin{array}{l}\mathrm{Fd}=0.99042+0.54015 * \mathrm{f}-5.67014 * 10^{-004 * V+} \\
0.057180 * \mathrm{~d}+7.87500 * 10^{-004} \mathrm{f} * \mathrm{~V}+0.22813 * \mathrm{f} * \mathrm{~d}- \\
8.50000 * 10^{-005} \mathrm{~V} * \mathrm{~d}-0.51989 *(\mathrm{f})^{2}+5.38909 * 10^{-006} \\
*(\mathrm{~V})^{2}-0.11818 *(\mathrm{~d})^{2}\end{array}$}} \\
\hline & & & \\
\hline & & & \\
\hline & & & \\
\hline & \multicolumn{3}{|l|}{$V(\mathrm{~m} / \mathrm{min}) ; f(\mathrm{~mm} /$ diente $) ; d(\mathrm{~mm})$} \\
\hline \multirow{4}{*}{$\begin{array}{l}\text { Praveen P. et } \\
\text { al. [61] } \\
\text { (ranurado) }\end{array}$} & \multicolumn{2}{|c|}{ a) $F d=1.18+1.07 * 10^{-3} \mathrm{~V}+8.6 * 10^{-5} \mathrm{f}+5.13^{*} 10^{-2} \mathrm{~d}$} & a) Ti-N coated \\
\hline & \multicolumn{2}{|c|}{ b) $\mathrm{Fd}=0.991+9.63 * 10^{-3} \mathrm{~V}+2.57 * 10^{-4} \mathrm{f}-5.13^{*} 10^{-2} \mathrm{~d}$} & b) Carburo \\
\hline & \multicolumn{2}{|c|}{ c) $\mathrm{Fd}=1.18+1.07 * 10^{-3} \mathrm{~V}+8.6^{*} 10^{-5} \mathrm{f}+5.13 * 10^{-2} \mathrm{~d}$} & c) Tipped carb \\
\hline & \multicolumn{2}{|l|}{$V(\mathrm{~m} / \mathrm{min}) ; f(\mathrm{~mm} / \mathrm{minuto}) ; d(\mathrm{~mm})$} & \\
\hline \multirow{3}{*}{$\begin{array}{l}\text { Davim J.P. et } \\
\text { al [48] } \\
\text { (ranurado) }\end{array}$} & a) $\mathrm{Fd}=1.00-6.58 * 10^{-5} \mathrm{~V}+2.34 * 10^{-5} \mathrm{f}$ & $\mathrm{R}=0.89$ & a) Dos filos \\
\hline & b) $\mathrm{Fd}=1.01-2.58^{*} 10^{-3} \mathrm{~V}+2.73 * 10^{-5} \mathrm{f}$ & $\mathrm{R}=0.67$ & b) Seis filos \\
\hline & \multicolumn{3}{|l|}{$V(\mathrm{~m} / \mathrm{min}) ; f(\mathrm{~mm} / \mathrm{minuto})$} \\
\hline \multirow{2}{*}{$\begin{array}{l}\text { Sheikh-Ahmad } \\
\text { J.et al. [74] } \\
\text { (contorneado) }\end{array}$} & \multicolumn{3}{|c|}{$\operatorname{DEL}(\mathrm{mm})=0.0905-0.000987 * \mathrm{~V}+0.0349 * \mathrm{f}+0.0104 * \mathrm{~L}$} \\
\hline & \multicolumn{3}{|l|}{$V(\mathrm{~m} / \mathrm{min}) ; f(\mathrm{~m} /$ minuto $) ; L(\mathrm{~m})$} \\
\hline
\end{tabular}

\subsection{Orientación de las fibras}

En el delaminado la influencia del ángulo de orientación de la fibra con la dirección del mecanizado se ha abordado definiendo ángulos que aúnan la orientación de la fibra con los movimientos de corte (Mc) y de avance (Ma). Estos ángulos son el ángulo de orientación de fibra (fiber orientation angle), el ángulo de corte (fiber cutting angle) y el ángulo de empañe (engagement angle), y se han definido tanto para la operación de ranurado como para la de contorneado. En la primera de ellas, el fresado se realiza en concordancia y oposición, y en la segunda se puede seleccionar el modo de fresado (oposición o concordancia). Los diferentes autores han llegado a conclusiones distintas sobre la aparición del delaminado y las zonas donde se produce.

En general, como podemos observar en la Figura 1.24, el ángulo de orientación de fibra $(\Theta)$, posiciona la orientación de la fibra respecto a la trayectoria de corte, el ángulo de empañe $(\phi)$ relaciona la dirección de los movimientos fundamentales y el ángulo de corte $(\chi)$ relaciona la orientación de la fibra con la velocidad de corte. 


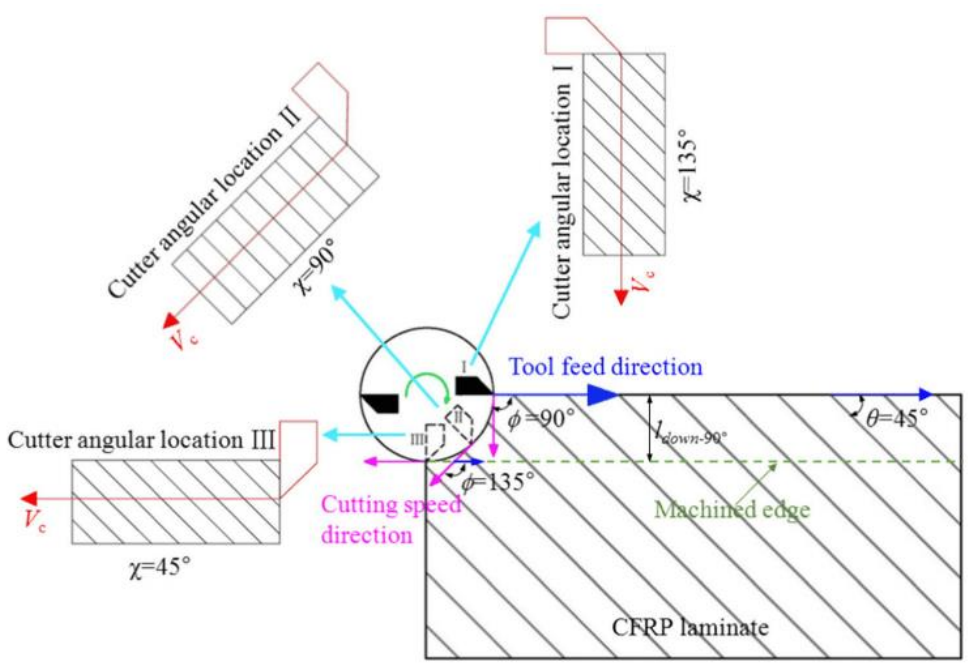

Figura 1.24. Ángulos relacionados con la orientación de la fibra en fresado. [70]

Aunque se ha trabajado con estos tres ángulos, no existe consenso entre los autores sobre la nomenclatura utilizada, su definición y la relación que existe entre ellos a la hora de explicar el efecto de la orientación de la fibra en el delaminado. La Tabla 1.10 muestra las diferencias entre los autores que utilizan en su argumentación alguno de estos ángulos.

Tabla 1.10. Definición ángulos de orientación de fibra para delaminado

\begin{tabular}{|c|c|c|c|c|}
\hline Referencias & $\begin{array}{c}\text { Ángulo de } \\
\text { orientación } \\
\text { de fibra }\end{array}$ & $\begin{array}{c}\text { Ángulo } \\
\text { de empañe }\end{array}$ & $\begin{array}{l}\text { Ángulo } \\
\text { de corte }\end{array}$ & Relaciones \\
\hline $\begin{array}{l}\text { Hintze W. et al. } \\
\text { [72] }\end{array}$ & $\phi$ & -- & $\Theta$ & No hay propuesta \\
\hline $\begin{array}{l}\text { Geis T. et al. } \\
\text { [79] }\end{array}$ & $\phi$ & $\varphi$ & $\Theta$ & No hay propuesta \\
\hline $\begin{array}{l}\text { He Y. et al. } \\
{[92]}\end{array}$ & $\psi$ & $\Theta$ & $\rho$ & $\rho=\psi+\Theta$ \\
\hline $\begin{array}{l}\text { Voss R. et al. } \\
{[67]}\end{array}$ & $\Theta$ & $\varphi$ & $\phi$ & $\begin{array}{l}\varphi=180-(\phi+\Theta) \\
\operatorname{para} 0^{\circ}=\phi+\Theta<180^{\circ} \\
\varphi=360-(\phi+\Theta) \\
\operatorname{para} 180^{\circ}=\phi+\Theta<360^{\circ}\end{array}$ \\
\hline $\begin{array}{l}\text { Wang F. et al. } \\
\text { [70] }\end{array}$ & $\Theta$ & $\phi$ & $\chi$ & $\begin{array}{ll}\chi=180+(\Theta-\varphi) \\
\quad & \text { para } \Theta-\varphi \leq 0 \\
\chi=\Theta-\varphi \quad & \text { para } \Theta-\varphi>0\end{array}$ \\
\hline $\begin{array}{l}\text { Wang D. et al. } \\
{[71]}\end{array}$ & $\Theta$ & $\varphi$ & $\beta$ & $\begin{array}{ll}\beta=\Theta-\phi & \text { para } \phi \leq \Theta \\
\beta=180-(\phi-\Theta) & \text { para } \phi>\Theta\end{array}$ \\
\hline
\end{tabular}


En todos ellos se asume que el movimiento de avance lo lleva la herramienta y no la pieza. Hintze W. et al., Geis T. et al., Voss R. et al. y Wang D. et al. $[67,71,75,78]$ definen el ángulo de la orientación de fibra desde el movimiento de avance hasta la dirección de la fibra, mientas que Wang F. et al. [70] lo definen al revés. Algo similar sucede con el ángulo de corte. Hintze W. et al. [75] y Geis T. et al. [79] lo definen desde el movimiento de corte a la dirección de la fibra y Voss R. et al. [67] y Wang F. et al. [70], al contrario. Todas estas diferencias hacen que la comparación de resultados entre ellos no sea fácil, y por eso plantean relaciones distintas entre los ángulos definidos 0 que se cumplen en rangos angulares diferentes.

\section{Ranurado}

En la operación de ranurado, Hintze W. et al. [72], han observado que el delaminado se produce entre los ángulos de orientación de fibra de $90^{\circ}$ y $180^{\circ}$ (Figura 1.25).

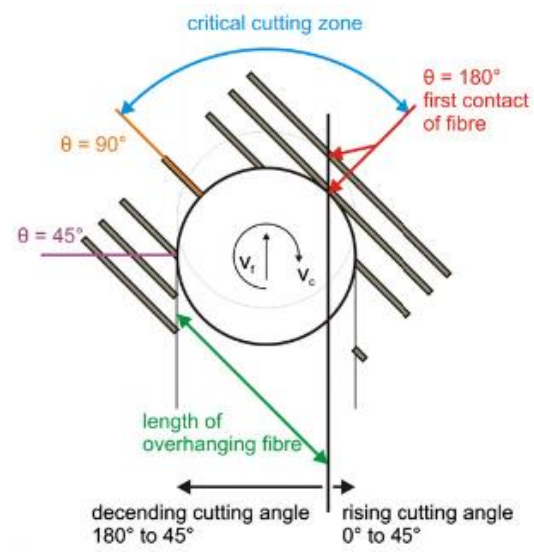

Figura 1.25. Zona crítica de inicio del delaminado [72].

Li M. et al. [56] evalúan el delaminado tipo II en laminados unidireccionales con orientación $0^{\circ} / 90^{\circ}$ y $45^{\circ} / 135^{\circ}$ y en tejidos. En este estudio se aprecia que en los tejidos unidireccionales con orientación $45^{\circ} / 135^{\circ}$ se produce más delaminado, con gran longitud de las fibras deshilachadas, que en el de orientación $0^{\circ} / 90^{\circ}$. En los tejidos bidireccionales el delaminado es mucho menor.

Liu G. et al. [68] afirman que en la operación de ranurado cuando se mecaniza con un ángulo de corte de fibra entre $0^{\circ}$ y $90^{\circ}$ se produce mucho delaminado del tipo II, mientras que si este ángulo se encuentra entre $90^{\circ} \mathrm{y}$ $180^{\circ}$ se produce un delaminado de tipo I. Sin embargo, Neeli N. et al. [76] afirman que la mejor orientación de las fibras es de $15^{\circ}$ para obtener un factor 
de delaminación más adecuado. Por último, He Y. et al. [49] afirman que el ángulo de la fibra de corte crítico es $90^{\circ}$, ya que se produce delaminado como consecuencia del doblado de las fibras.

\section{Contorneado}

Para la operación de contorneado hay menos trabajos publicados, Wang F. et al. [70] con la definición de ángulos de la Figura 1.24, concluyen que el delaminado tipo II se produce en ángulos de corte de fibras entre $0^{\circ}$ y $90^{\circ}$ y con radios de punta grandes.

Wang D. et al. [71] en sus estudios de contorneado, afirman que aparece delaminado de tipo II entre los ángulos de corte de las fibras de $0^{\circ}$ a $135^{\circ}$ y que, además, entre los ángulos de $90^{\circ}$ y $135^{\circ}$ aparece delaminado de tipo I. Como condiciones favorables para disminuir el delaminado, proponen trabajar en concordancia para un ángulo de orientación de fibra de $0^{\circ}$ y $90^{\circ}$, y en oposición para $45^{\circ}$ y $135^{\circ}$.

Ozkan D. et al. [60], realiza el mecanizado del borde de una pieza e indica que cuando trabaja con materiales con una orientación de fibra de $0^{\circ}$ y de $90^{\circ}$, la pieza y la herramienta no presentan daños, sin embargo al trabajar con una orientación de fibra de $\pm 45^{\circ}$ aparecen elevados daños sobre la pieza durante el fresado.

\subsection{Herramienta}

Las características de las herramientas en el delaminado han sido estudiadas por la comunidad científica. En concreto se ha analizado principalmente la influencia del material de la herramienta, el número de filos, el radio de punta y el ángulo de inclinación. De todas formas, los autores coinciden en afirmar que estas variables tienen menos influencia en el delaminado que las expuestas anteriormente.

En lo que respecta al material de la herramienta, Praveen P. et al. [61], indica que las herramientas con recubrimiento provocan menos daño en la pieza que las herramientas de carburo cementado sin recubrimiento .

Respecto al número de filos (z), esta variable se ha estudiado en operaciones de ranurado, midiendo un factor de delaminación para cuantificar el delaminado. Las conclusiones han sido contradictorias entre los diferentes autores. Hay autores que afirman que el factor de delaminado disminuye cuando la herramienta tiene un número menor de filos, [48, 62], mientras que 
otros afirman lo contrario, que el factor de delaminado disminuye si la herramienta tiene mayor número de filos, [51, 57].

Hay pocas referencias que analicen el efecto del radio de punta de la herramienta. En uno de los pocos estudios que aborda esta variable, se concluye que radios de punta grandes provocan más delaminado en la pieza [70].

Sobre el ángulo de inclinación, en el estudio de Neeli N. et al. [76], se concluye que el factor de delaminado aumenta conforme aumenta el ángulo de inclinación de la herramienta.

\subsection{Parámetro $X d$}

De los trabajos que estudian el delaminado, destacan los de Hintze W. et al. [72, 75, 78, 88, 92-93]. En sus trabajos no se aborda la influencia de las condiciones de corte, pero sí otros parámetros para intentar explicar cómo se inicia y propaga el delaminado, así como los tipos de delaminado observados y la longitud de las fibras delaminadas.

En los tejidos formados por la trama y la urdimbre, Hintze W. et al. [75] define el parámetro Xd como la distancia desde el borde mecanizado de la pieza hasta el cruce con la siguiente hebra del tejido. De esta forma, el parámetro Xd varía, si la trayectoria de la herramienta al mecanizar, no es paralela al cruce de las hebras (Figura 1.26). Esta variación provoca un diferente delaminado en la pieza mecanizada.

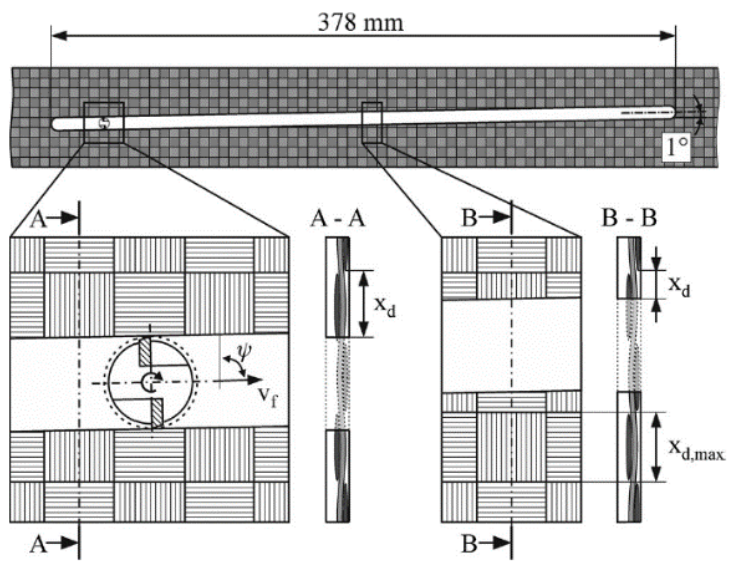

Figura 1.26. Parámetro Xd [75].

En la capa superior del laminado, donde se produce la delaminación, se pueden distinguir tres zonas, teniendo en cuenta la ondulación del tejido y el 
espesor de la resina (Figura 1.27). En cada zona se pueden identificar delaminados de diferentes magnitudes y tipos (tipos I y II). Así, si el parámetro $\mathrm{Xd}$ aumenta se produce un delaminado mayor de tipo I, mientras que el de tipo II disminuye.

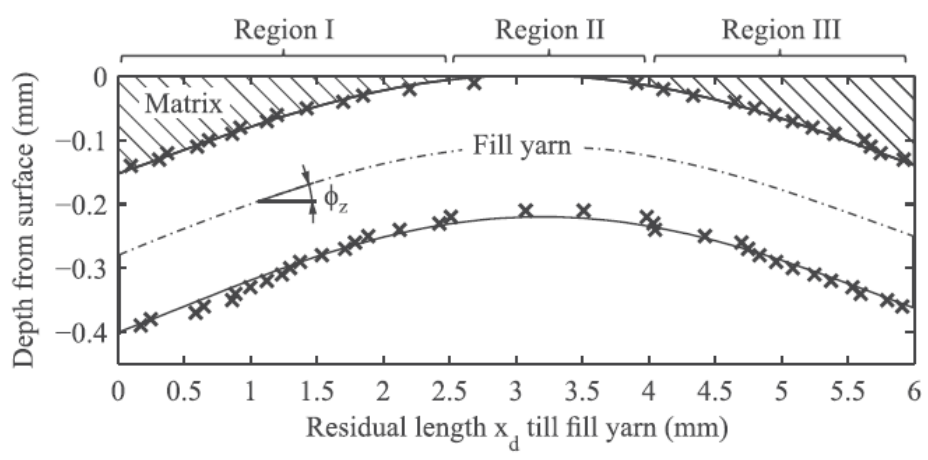

Figura 1.27. Regiones en la capa superior del laminado [75].

\subsubsection{TÉCNICAS DE OPTIMIZACIÓN APLICADAS}

La mayoría de los trabajos que abordan el estudio del delaminado, la rugosidad superficial, el desgaste de herramienta y las fuerzas generadas en el mecanizado proponen modelos empíricos mediante la técnica de regresión, completando con un análisis de varianzas (ANOVA). Algunos trabajos utilizan diferentes técnicas de optimización, como las redes neuronales o algoritmos genéticos, para predecir los valores de las variables como la rugosidad o el delaminado.

Las redes neuronales son modelos computacionales consistentes en grupos de nodos interconectados para transmitir señales, similares a la red de neuronas de un cerebro. La información de entrada atraviesa la red neuronal, donde se somete a determinadas operaciones, para producir unos valores de salida. Sreenivasulu R. et al. [64] aplican redes neuronales para predecir la rugosidad superficial y el factor de delaminado en operaciones de ranurado, tomando como variables de entrada las condiciones de corte (velocidad, avance y profundidad). Erkan Ö. et al. [57] utilizan redes neuronales para predecir el factor de delaminado en la operación de ranurado, tomando como variables de entrada el número de dientes de la herramienta y las condiciones de corte (velocidad, avance y profundidad). En este trabajo se evalúan diferentes algoritmos de aprendizaje, además de modificar el número de neuronas del sistema.

Los algoritmos genéticos son algoritmos utilizados para resolver problemas de búsqueda y optimización, basados en la evolución natural y la genética de 
una población de seres vivos. Existen trabajos que combinan estos algoritmos junto con las redes neuronales. Razfar M.R. et al. [62] utilizan algoritmos genéticos y redes neuronales con el objetivo de minimizar la rugosidad superficial y el factor de delaminación, teniendo en cuenta las condiciones de corte y el número de filos de la herramienta. Robbany F. et al. [69] utilizan también estas dos técnicas conjuntamente para predecir la fuerza generada durante el corte y el factor de delaminación tomando como variables de entrada las condiciones de corte, como en el resto de los trabajos. 


\section{CAPÍTULO 2. MATERIALES Y MÉTODOS}

\subsection{INTRODUCCIÓN}

En este capítulo se van a presentar los materiales y métodos utilizados para el desarrollo de los experimentos llevados a cabo en la tesis doctoral. Los laminados están compuestos por tejido de basalto y resina epoxi y se han fabricado mediante moldeo por transferencia de resina (RTM), variando tanto el porcentaje como la orientación de la fibra. Posteriormente, estas piezas se han mecanizado en una fresadora vertical de control numérico mediante la operación de contorneado.

Tras el mecanizado se ha evaluado la calidad de los laminados y del proceso, midiendo la rugosidad superficial, el desgaste de la herramienta y evaluando los tipos de delaminado del material (Figura 2.1) para cada uno de los experimentos, en diferentes tiempos de corte. 


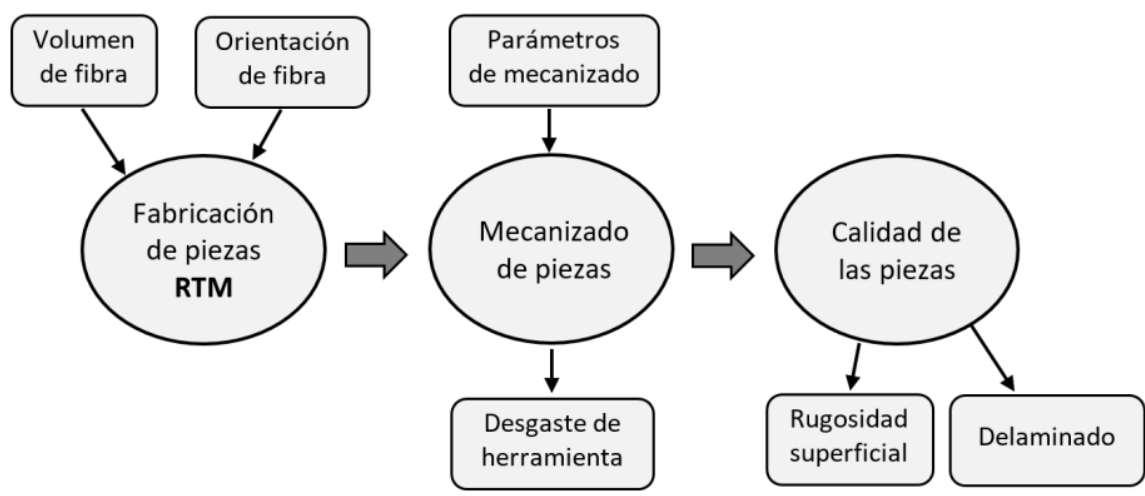

Figura 2.1. Esquema del procedimiento.

\subsection{FABRICACIÓN DE LOS LAMINADOS POR RTM}

Las piezas a fabricar son laminados de matriz polimérica con refuerzo de fibra y fabricadas por RTM (resin transfer molding). El refuerzo elegido es un tejido bidireccional de fibra larga de basalto, y la matriz una resina epoxi para infusión/RTM de baja viscosidad (Tabla 2.1). Las fichas de seguridad y características de estos componentes se presentan en los Anexos I.1 y I.2.

Tabla 2.1. Características del material: refuerzo y matriz.

\begin{tabular}{ll}
\hline Refuerzo & \\
\hline Nombre comercial & FABRIC TYPE TBK- 400 \\
\hline Fabricante & Basaltex \\
\hline Distribuidor & Juan Gili S.L. \\
\hline Espesor & $0.4 \mathrm{~mm}$ \\
\hline Densidad & $400 \mathrm{~g} / \mathrm{m}^{2}$ \\
\hline Tipo Tejido & Plain weave \\
\hline
\end{tabular}

\begin{tabular}{|c|c|}
\hline Matriz & \\
\hline Nombre comercial & PRIMETM 20LV \\
\hline Fabricante & Gurit \\
\hline Distribuidor & Resinas Castro \\
\hline
\end{tabular}

El material compuesto se ha fabricado en un molde rectangular de aluminio mecanizado previamente (Figura 2.2). El molde consta de dos semimoldes planos, superior e inferior, con un sistema de cierre a base de tornillos repartidos uniformemente a lo largo de todo el borde, y dos juntas tóricas para 
garantizar la estanqueidad. El semimolde superior dispone de válvulas de entrada y salida para llevar a cabo el proceso de llenado por RTM.

El molde dispone de un marco interior intercambiable que delimita la forma del laminado y permite fabricar piezas rectangulares de $260 \mathrm{~mm}$ x $500 \mathrm{~mm}$ y espesor constante de $3.4 \mathrm{~mm}$ (Figura 2.3).

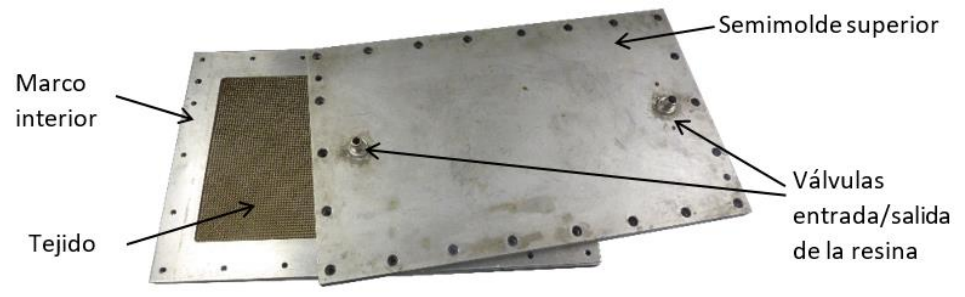

Figura 2.2. Molde para la fabricación por RTM.

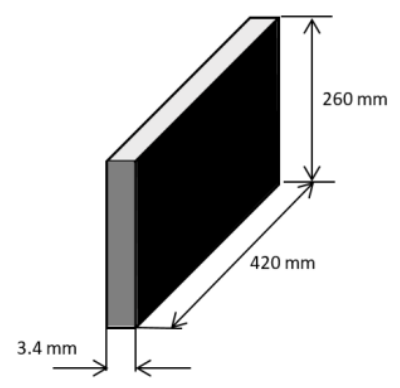

Figura 2.3. Placa fabricada por RTM.

El tejido de basalto se introduce en el interior del molde, y con ayuda de un calderín intermedio y un regulador de presión se controla la entrada de la resina catalizada para el llenado de las piezas, a presión constante (Figura 2.4). Posteriormente, se realiza el curado siguiendo las instrucciones del proveedor de la resina.

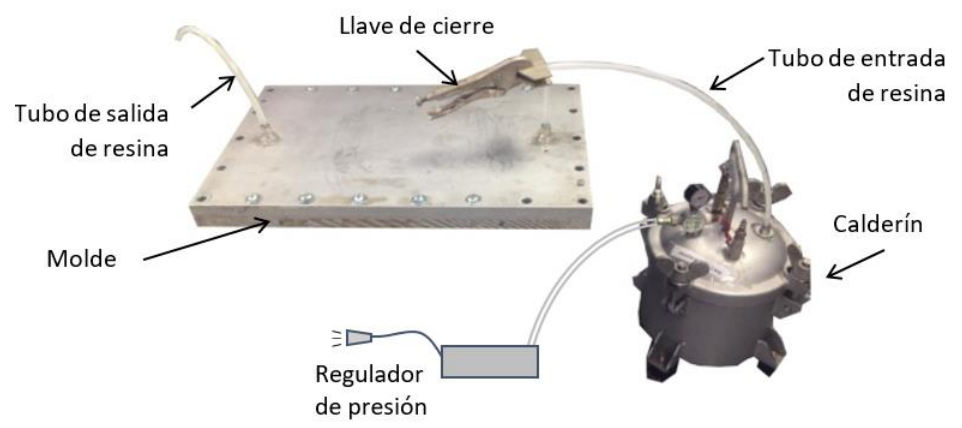

Figura 2.4. Montaje de equipos para la fabricación de los laminados. 


\subsection{MECANIZADO DE LOS LAMINADOS}

Una vez fabricados los laminados de material compuesto se mecanizan en una fresadora vertical de control numérico de tres ejes, cuyas características se adjuntan en el Anexo I.3. El mecanizado consiste en realizar un fresado periférico en concordancia, sin lubricación, a lo largo del borde del laminado. Este mecanizado es equivalente al recorte que sufre una pieza convencional de material compuesto en el sector industrial para ajustarla a las dimensiones finales del producto, antes de su ensamblaje (Figura 2.5), donde el movimiento de corte (Mc) lo lleva la herramienta, el movimiento de avance (Ma) lo lleva la pieza, el ángulo de contacto entre la herramienta y la pieza o ángulo de fresado está representado por $\Theta$ y la profundidad de pasada por $a_{p}$.

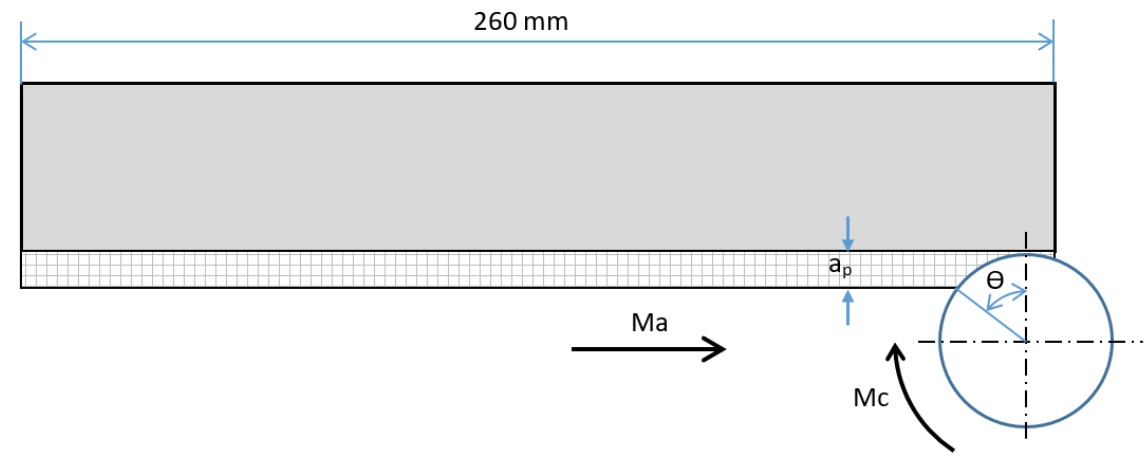

Figura 2.5. Esquema del proceso de mecanizado.

\subsubsection{AMARRE}

El amarre del laminado en la máquina es uno de los puntos críticos de los experimentos. Al mecanizar laminados con espesor pequeño, es necesario diseñar un sistema de amarre robusto que evite la vibración del laminado durante el proceso de corte.

Los elementos de amarre se han dispuesto en la máquina de forma que se pueda sujetar el laminado a lo largo de todo el borde mecanizado, con muy poco voladizo para garantizar su estabilidad. Para ello, se han utilizado dos regles uno superior y otro inferior que amarran la pieza sujetándola por ambos lados. Además, se han utilizado dos topes de referencia que facilitan el posicionamiento y el cambio ágil de los laminados. Se ha fabricado también una galga para el control del voladizo en cada cambio de laminado (Figuras 2.6 y 2.7 ). 


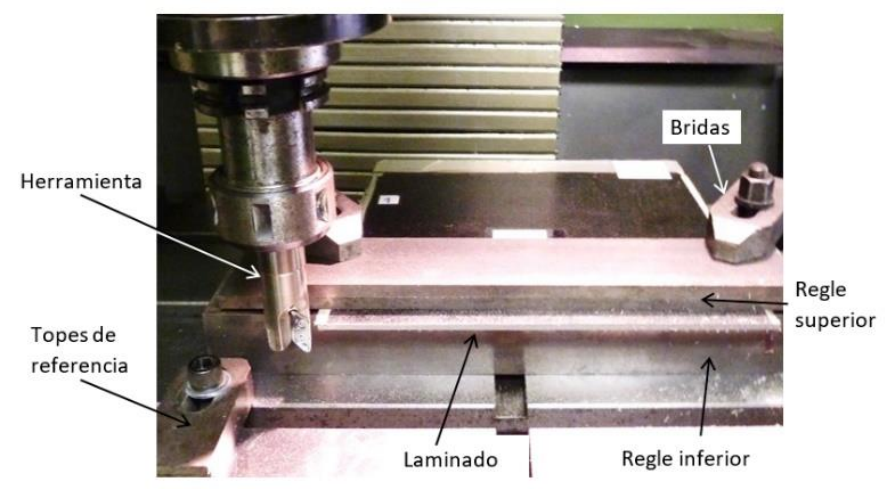

Figura 2.6. Elementos de sujeción para el amarre de los laminados.

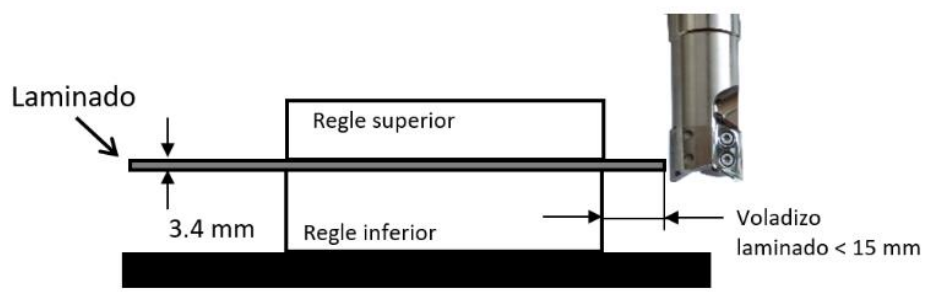

(a)

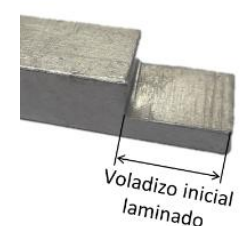

(b)

Figura 2.7. (a) Máximo voladizo del laminado (b) Galga de control.

Con este amarre, toda la longitud del laminado queda libre para realizar el fresado periférico del borde, permitiendo ajustar la profundidad de pasada $\left(a_{p}\right)$ en función de las condiciones de mecanizado de los experimentos planteados (Figura 2.8).
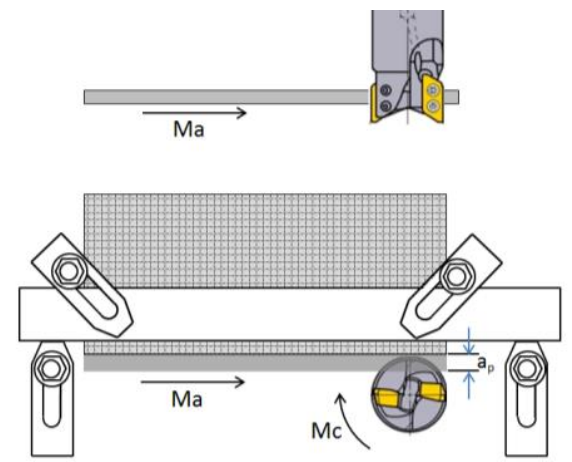

Figura 2.8. Esquema del fresado periférico y amarre en la máquina. 
El material que se va a mecanizar es muy abrasivo, y la fresadora de control numérico no dispone de un sistema de aspiración de viruta. Para evitar el efecto abrasivo de la viruta o polvo de fibra de basalto sobre la máquina, se prepara una bolsa de sellado que envuelve y recoge toda la viruta producida (Figura 2.9), permitiendo a la vez todos los movimientos necesarios del cabezal y la mesa de la máquina. De esta forma se pueden hacer los experimentos de forma estanca.

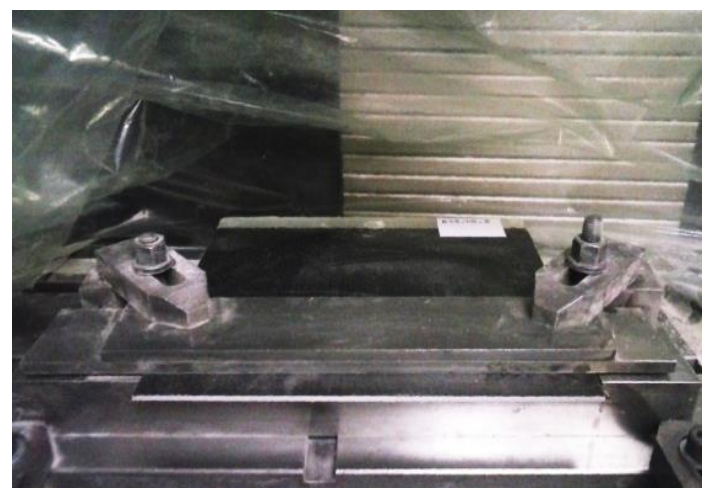

Figura 2.9. Detalle de la bolsa de sellado.

\subsubsection{SELECCIÓN DE LA HERRAMIENTA DE CORTE}

Los experimentos se han realizado con una herramienta comercial, de geometría favorable y un precio adecuado frente a su rendimiento, permitiendo realizar el estudio del desgaste en el mecanizado del laminado reforzado con basalto.

La herramienta de corte seleccionada es una fresa de $25 \mathrm{~mm}$ de diámetro (AXD4000R252SA25SA), con dos plaquitas intercambiables (XDGX 175008PDFR-GL-TF15), del fabricante Mitsubishi Materials [95-96] (Figura 2.10). El plano de la herramienta y sus características se adjuntan en el Anexo I.4.

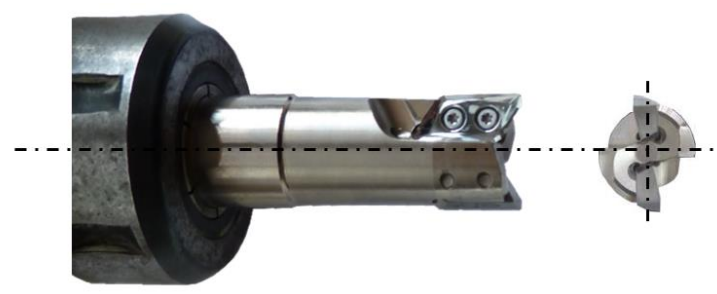

Figura 2.10. Detalle de la herramienta. 
Esta herramienta pertenece al grupo de herramientas de carburo sinterizado, sin recubrimiento (calidad TF15), presentando un comportamiento en cuanto a dureza, tenacidad y resistencia. Para cumplir con los requisitos de mecanizado de este tipo de materiales, dentro del tipo de herramientas de plaquitas intercambiables, se ha elegido una gama con filo de corte muy agudo (tipo GL), un ángulo de inclinación de $15^{\circ}$ y un ángulo de desprendimiento positivo de $25^{\circ}$ (Figura 2.11).

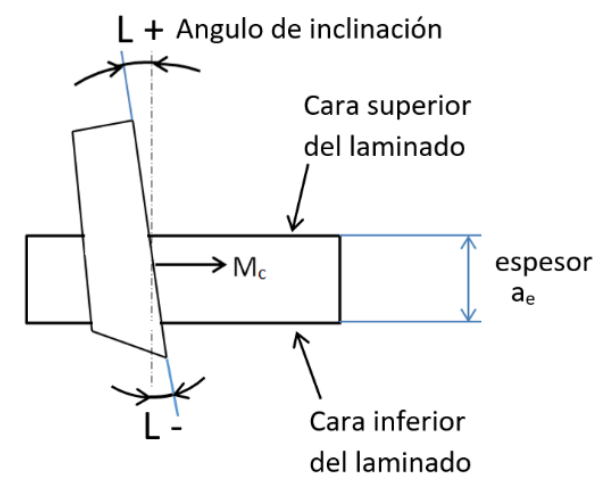

Figura 2.11. Angulo de inclinación de la herramienta.

\subsection{PLANTEAMIENTO DE LOS EXPERIMENTOS}

El estudio del mecanizado del material compuesto conlleva la decisión de decidir qué variables se van a evaluar. El estudio de un elevado número de variables implica un consumo elevado de material y de tiempo para llevar a cabo los experimentos. Para ajustar el número de experimentos, se ha llevado a cabo un diseño de experimentos (DOE). Para ello se han definido las variables de entrada y salida que se han considerado más influyentes y, de esta forma, se ha podido reducir el número de experimentos a realizar.

\subsubsection{VARIABLES DE ENTRADA}

Las variables de entrada incluyen tanto las condiciones de corte como las variables que afectan al material de la pieza. Las condiciones de corte a estudiar son la velocidad de corte $(\mathrm{Vc})$, el avance por diente $(\mathrm{Fz})$ y la profundidad de pasada $\left(a_{p}\right)$. Los valores de estas variables se han elegido dentro de un rango lo suficientemente amplio para poder estudiar su efecto en el mecanizado del material compuesto. En su elección se han tenido en cuenta, principalmente, las limitaciones por el tipo de herramienta, la máquina de C.N. y el material a mecanizar. 
Con respecto a las variables que afectan al material del laminado se van a evaluar el volumen de fibra $(\mathrm{Fv})$ y la orientación de fibra con respecto a la dirección del mecanizado (Fo).

Tanto las condiciones de corte como el volumen y la disposición del tejido en el material son parámetros fundamentales para este estudio, ya que van a influir en el desgaste de la herramienta, en el acabado superficial del laminado y en el delaminado.

Evidentemente, existen otras variables que también podrían ser evaluadas, como la geometría y el material de la herramienta, pero debido a lo costoso que resulta realizar este tipo de experimentos, se ha tenido que reducir su número en la medida de lo posible.

Inicialmente, para las variables elegidas se planteó su estudio en dos y tres niveles, como se muestra en la Tabla 2.2.

Tabla 2.2. Variables de entrada. Niveles definidos del diseño de experimentos.

\begin{tabular}{lccc}
\hline & Nivel 1 & Nivel 2 & Nivel 3 \\
\hline Vc $(\mathrm{m} / \mathrm{min})$ & 300 & 385 & 470 \\
\hline Fz $(\mathrm{mm} /$ diente $)$ & 0.1 & 0.25 & 0.4 \\
\hline ap $(\mathrm{mm})$ & 0.5 & 1.5 & \\
\hline Fo $\left({ }^{\circ}\right)$ & $0^{\circ}-90^{\circ}$ & $45^{\circ}-135^{\circ}$ & \\
\hline Fv $(\%)$ & $40 \%$ & $60 \%$ & \\
\hline
\end{tabular}

\subsubsection{VARIABLES DE SALIDA}

Las variables de salida que se van a evaluar en los experimentos son:

- El acabado superficial, a través de la medida del parámetro de rugosidad $\mathrm{Ra}$ (rugosidad media aritmética del perfil) en los laminados mecanizados.

- El desgaste de la herramienta, a través de la medida del desgaste en la cara de incidencia o $\mathrm{Vb}$ (desgaste de flanco).

- El delaminado de la pieza, que es un problema muy frecuente en el mecanizado de materiales compuestos con fibras, y que requiere la definición y el control de varios parámetros para cuantificar su influencia.

Estas variables van cambiando sus valores, conforme aumenta el tiempo de mecanizado. Por ello, en cada experimento se han realizado varias paradas a 
diferentes tiempos de corte, y se han evaluado cada una de las variables de salida, lo que ha incrementado notablemente la toma de datos. Estas paradas se han establecido a tiempos de mecanizado fijos de $5,30,50,60,70$ y 80 minutos, salvo para la medida del delaminado, donde no se han tomado datos para $\mathrm{Tc}=5$ min por ser el delaminado muy pequeño. De esta forma, al mismo tiempo se obtienen los resultados de los experimentos a lo largo del tiempo.

\subsubsection{DISEÑO DE EXPERIMENTOS}

El diseño de experimentos se ha realizado con ayuda del software Statgraphics. Al plantear el diseño de experimentos con las variables y niveles elegidos, la opción de un diseño factorial resultaba muy costosa sobre todo en consumo de material y en tiempo de mecanizado. Por este motivo, se ha propuesto realizar un diseño de matriz ortogonal L16, sin réplicas para reducir el número de experimentos.

El diseño de experimentos planteado se adjunta en la Tabla 2.3, y requiere un total de 16 experimentos.

Tabla 2.3. Valores de las variables de entrada por experimento.

\begin{tabular}{cccccc}
\hline Exp & $\begin{array}{c}\mathrm{Vc} \\
\mathrm{m} / \mathrm{min}\end{array}$ & $\begin{array}{c}\mathrm{Fz} \\
\mathrm{mm} / \mathrm{diente}\end{array}$ & $\begin{array}{c}\mathrm{ap} \\
\mathrm{mm}\end{array}$ & $\begin{array}{c}\mathrm{Fv} \\
\%\end{array}$ & $\begin{array}{c}\text { Fo } \\
\text { o }\end{array}$ \\
\hline 01 & 470 & 0.1 & 1.5 & 40 & 90 \\
\hline 02 & 300 & 0.1 & 1.5 & 40 & 45 \\
\hline 03 & 300 & 0.4 & 0.5 & 40 & 45 \\
\hline 04 & 300 & 0.4 & 1.5 & 60 & 45 \\
\hline 05 & 470 & 0.1 & 0.5 & 60 & 90 \\
\hline 06 & 470 & 0.4 & 0.5 & 40 & 90 \\
\hline 07 & 300 & 0.1 & 0.5 & 60 & 45 \\
\hline 08 & 470 & 0.4 & 0.5 & 60 & 45 \\
\hline 09 & 470 & 0.1 & 0.5 & 40 & 45 \\
\hline 10 & 300 & 0.1 & 0.5 & 40 & 90 \\
\hline 11 & 300 & 0.1 & 1.5 & 60 & 90 \\
\hline 12 & 300 & 0.4 & 0.5 & 60 & 90 \\
\hline 13 & 470 & 0.4 & 1.5 & 40 & 45 \\
\hline 14 & 470 & 0.4 & 1.5 & 60 & 90 \\
\hline 15 & 300 & 0.4 & 1.5 & 40 & 90 \\
\hline 16 & 470 & 0.1 & 1.5 & 60 & 45 \\
\hline & & & & & \\
\hline
\end{tabular}




\subsection{PROCEDIMIENTO DE MEDIDA DE LAS VARIABLES DE SALIDA}

La evaluación de las variables de salida (rugosidad, desgaste de la herramienta y delaminado), requiere de diferentes procedimientos y métodos de medida. A continuación, se van a desarrollar estos procedimientos de medida, que en todos los casos han requerido del diseño de utillajes o equipos especiales que permitan una medida fiable de cada una de las variables.

\subsubsection{PROCEDIMIENTO DE MEDIDA DE LA RUGOSIDAD SUPERFICIAL}

El parámetro de rugosidad superficial medido ha sido Ra (rugosidad media aritmética del perfil). La rugosidad superficial se ha medido con un rugosímetro modelo SJ-201 de Mitutoyo, cuyas características se encuentran en el Anexo I.5.

La zona mecanizada de un material compuesto presenta muchas irregularidades debido a la distinta naturaleza de sus componentes, donde se puede distinguir con claridad la resina de la fibra. Por este motivo, la medida de la rugosidad presenta diferencias importantes según el punto donde apoye el palpador del equipo al medir.

Para la medida de la rugosidad superficial, se ha diseñado un banco de medida que permita acceso para medir en la zona del espesor del laminado mecanizado, y al mismo tiempo actúe de soporte para el rugosímetro. En el banco diseñado, la placa a medir se coloca entre dos regles paralelos que la soportan, y que al mismo tiempo forman un plano de apoyo para el rugosímetro, garantizando la estabilidad del aparato durante el proceso de medida. De esta forma, toda la longitud mecanizada del laminado está completamente accesible para realizar cualquier tipo de medida (Figura 2.12). Estos regles llevan unas bridas o tornillos que permiten ajustar su separación en función del espesor del laminado a sujetar.

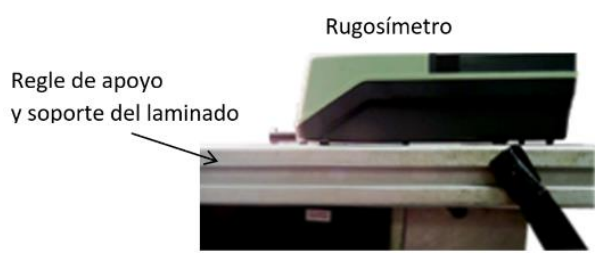

(a)

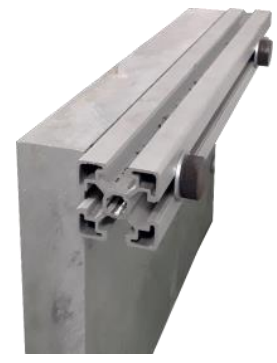

(b)

Figura 2.12. (a) Rugosímetro (b) Banco de medida de rugosidad. 
La rugosidad superficial se ha evaluado en sentido longitudinal, dado que, en sentido transversal, el laminado sólo se dispone de $3.4 \mathrm{~mm}$ de espesor. El procedimiento de medida utilizado para cada experimento evalúa el parámetro de rugosidad media aritmética del perfil ( $\mathrm{Ra}$ ) en tres posiciones a lo largo del borde mecanizado, dos de ellas cerca de los extremos y otra en el centro de la placa (Figura 2.13).

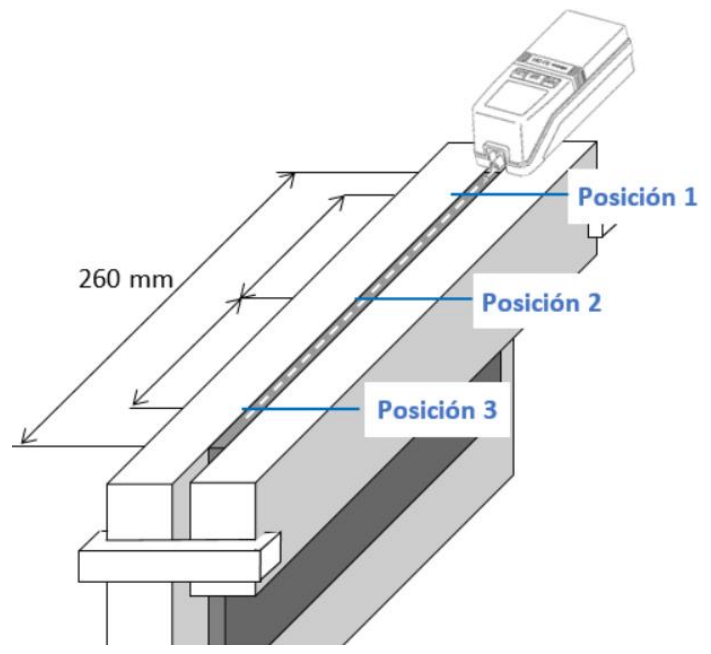

Figura 2.13. Posiciones de medida de rugosidad sobre el laminado.

En cada una de estas tres posiciones se toman cinco medidas repartidas de forma uniforme en la dirección del espesor del laminado, un total de quince medidas por experimento y tiempo de mecanizado (Figura 2.14). El valor de la rugosidad superficial para cada experimento se determina como la media de las quince medidas obtenidas.

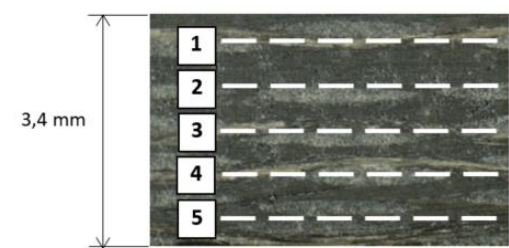

(a)

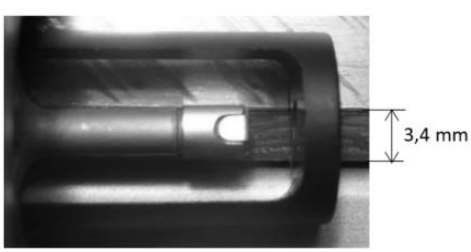

(b)

Figura 2.14. (a) Puntos de medida en el espesor de la placa. (b) Posición palpador. 


\subsubsection{PROCEDIMIENTO DE MEDIDA DEL DESGASTE DE LA HERRAMIENTA}

En este tipo de material y con este proceso de mecanizado, el desgaste de los filos de corte de la herramienta es bastante uniforme y se produce mayoritariamente sobre la cara de incidencia. Por ello, se va a evaluar el parámetro $\mathrm{Vb}$, en cada uno de los filos, mediante la captura de imágenes de ambos filos de corte. Las imágenes para evaluar el desgaste se toman fuera de la máquina y sin desmontar las plaquitas del portaherramientas, mediante la fotografía de ambos filos de corte. Las imágenes se analizan con un software desarrollado en Visual Basic y, posteriormente, mediante una hoja de cálculo, se realizan todos los cálculos geométricos necesarios para determinar el valor de $\mathrm{Vb}$.

Para la captura de estas imágenes se ha diseñado y fabricado un banco de medida que permite fotografiar los dos filos de corte manteniendo el plano de enfoque, el encuadre y la escala de una forma rápida. El banco dispone de un soporte fijo para la herramienta y de un soporte ajustable para la cámara. Como soporte de la herramienta, el banco tiene dos puntos de apoyo en el cono ISO y en el portaherramientas, hechos a medida para el mango de la herramienta, que garantizan la horizontalidad del eje de la herramienta (Figura 2.15 (a)).

Como soporte de la cámara, el banco de medida dispone de una base de posición ajustable mediante dos nonius de control de posicionamiento en los ejes X e Y (Figura 2.15 (b)), sobre la que se sujeta la cámara mediante un tornillo inferior. Este soporte permite ajustar la posición de la cámara respecto al plano de enfoque mediante los nonius.
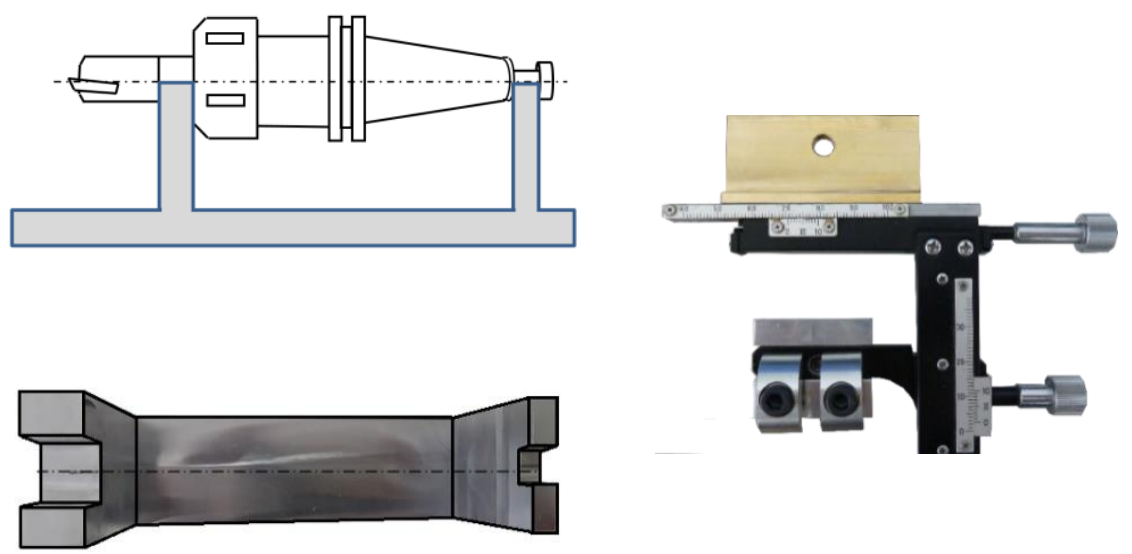

(a) Soporte de la herramienta

(b) Soporte de la cámara

Figura 2.15. Elementos del banco de medida de desgaste. 
El banco, una vez construido, junto con la herramienta a medir y la cámara, se muestra en la Figura 2.16.
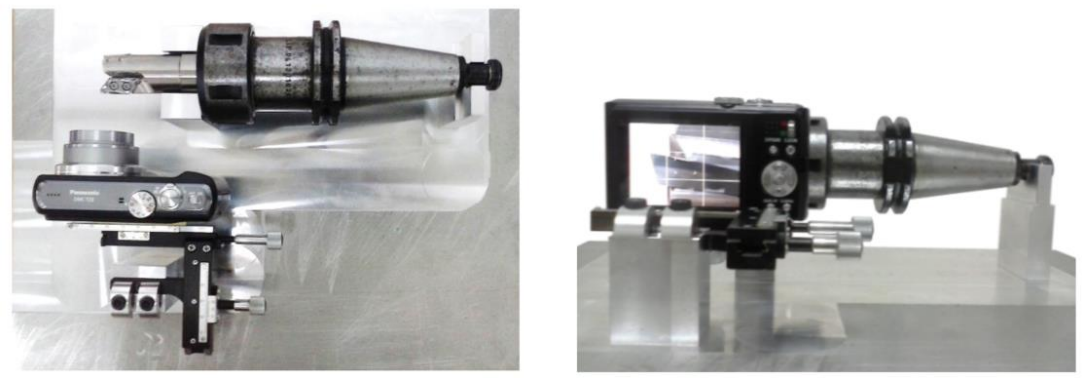

Figura 2.16. Banco de medida del desgaste de la herramienta.

Una vez tomadas las fotografías, para la captura de puntos sobre la imagen se ha desarrollado un software en Visual Basic (VB.NET), que permite almacenar y exportar estos puntos y sus referencias fácilmente. El software dispone de una ventana de registro de datos y otra ventana de imagen (Figura 2.17). La ventana de registro de datos muestra la información de los metadatos seleccionados en la pantalla de imagen.

En cada imagen procesada se han tomado las siguientes variables:

- Información general sobre la identificación del experimento, condiciones, tiempo de mecanizado, filos, etc.

- Referencias de cálculo de escala, con la medida de los patrones de referencia horizontal y vertical incluidos en la captura. Además de un punto posición o punto de referencia fijo de posicionamiento de la plaquita en el encuadre, común a todas las capturas.

- Valores de desgaste medidos a través de la medida del eje transversal y del eje longitudinal de desgaste observado (Figura 2.18). Estos ejes se definen a partir de las coordenadas (x,y) de sus extremos, capturados sobre la imagen. Estos valores, se pueden repetir y realizar tantas medidas como sea necesario en función de la situación particular de la imagen.

La ventana de imagen permite seleccionar con el ratón sobre la fotografía, variando la ampliación de la imagen, las coordenadas (x,y) de los puntos de interés (Figura 2.17). Estas coordenadas se asignan a las características programadas, para posteriormente exportar dicha información a un programa de cálculo, donde se realizan los cálculos geométricos para determinar el valor $\mathrm{de} \mathrm{Vb}$ en cada punto. 


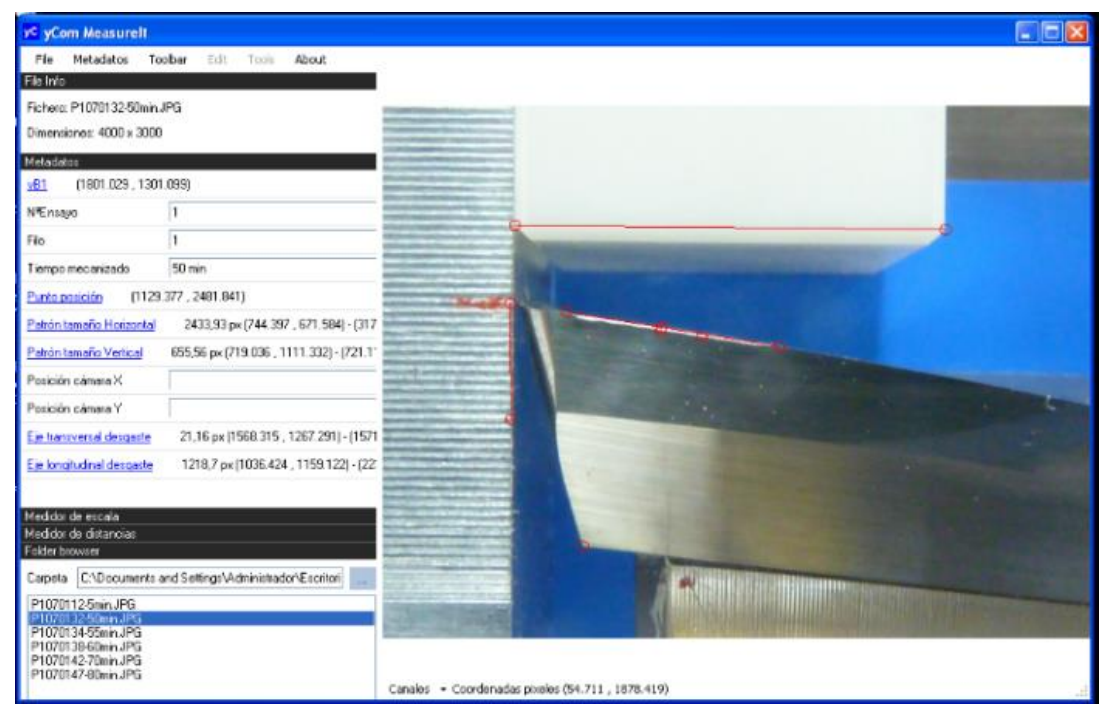

Figura 2.17. Ventana de registro de datos y ventana de imagen.

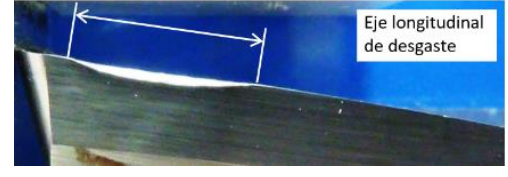

(a)

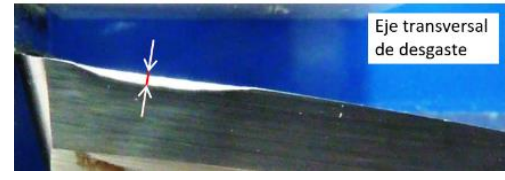

(b)

Figura 2.18. Eje longitudinal y transversal de desgaste.

Se toman dos valores de desgaste para cada tiempo de corte, Vb1 y Vb2, en posiciones fijas del filo cerca del centro del eje longitudinal (Figura 2.19). El valor de desgaste asignado a cada experimento y tiempo de corte es la mayor de las medidas realizadas. 


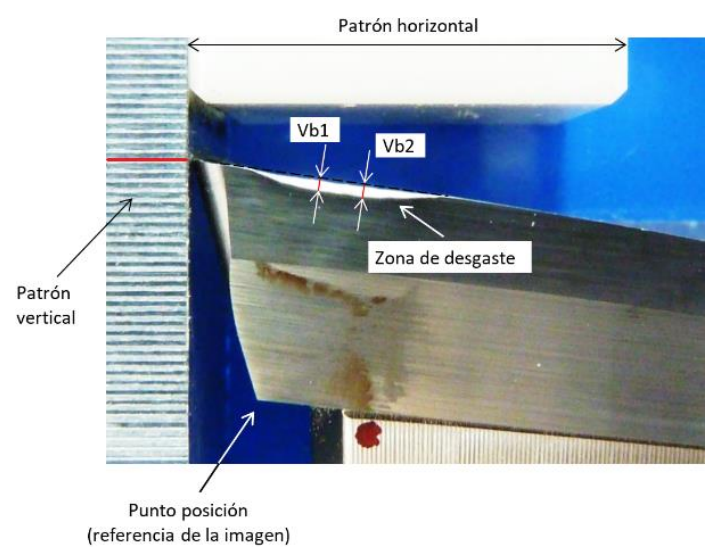

Figura 2.19. Detalle de los elementos de la imagen capturada.

La información de salida que ofrece el software es una tabla de datos, como la que se muestra en la Tabla 2.4.

Tabla 2.4. Ejemplo de registro de salida de datos-Medida desgaste E02.

\begin{tabular}{|c|c|c|c|c|c|c|c|c|c|c|c|c|}
\hline \multicolumn{13}{|l|}{ METADATO } \\
\hline NAME & \multicolumn{6}{|c|}{ Tiempo mecanizado } & \multicolumn{6}{|c|}{ Tiempo mecanizado } \\
\hline VALUE & 5 & 30 & 50 & 60 & 70 & 80 & 5 & 30 & 50 & 60 & 70 & 80 \\
\hline NAME & № Filo & \multicolumn{11}{|c|}{ № Filo } \\
\hline VALUE & 1 & 1 & 1 & 1 & 1 & 1 & 2 & 2 & 2 & 2 & 2 & 2 \\
\hline NAME & №Ensayo & \multicolumn{11}{|c|}{ №Ensayo } \\
\hline VALUE & 2 & 2 & 2 & 2 & 2 & 2 & 2 & 2 & 2 & 2 & 2 & 2 \\
\hline \multicolumn{13}{|l|}{ METADATO } \\
\hline TIPO & Punto & \multirow{2}{*}{\multicolumn{11}{|c|}{$\begin{array}{l}\text { Punto } \\
\text { vB1 }\end{array}$}} \\
\hline NAME & vB1 & & & & & & VB1 & & & & & \\
\hline $\mathrm{x}$ & $\begin{array}{c}1803.00 \\
0746\end{array}$ & $\begin{array}{c}1800.46 \\
7559\end{array}$ & $\begin{array}{c}1800.51 \\
1715\end{array}$ & $\begin{array}{c}1802.89 \\
6148\end{array}$ & $\begin{array}{c}1800.68 \\
8339\end{array}$ & $\begin{array}{c}1803.34 \\
9975\end{array}$ & $\begin{array}{c}1802.10 \\
1337\end{array}$ & $\begin{array}{c}1801.76 \\
5668\end{array}$ & $\begin{array}{c}1800.68 \\
8339\end{array}$ & $\begin{array}{c}1800.24 \\
6778\end{array}$ & $\begin{array}{c}1801.99 \\
5362\end{array}$ & $\begin{array}{c}1803.69 \\
0959\end{array}$ \\
\hline Y & $\begin{array}{c}1255.30 \\
7594 \\
\end{array}$ & $\begin{array}{c}1258.81 \\
8622 \\
\end{array}$ & $\begin{array}{c}1262.15 \\
9772 \\
\end{array}$ & $\begin{array}{c}1236.37 \\
2572 \\
\end{array}$ & $\begin{array}{c}1231.51 \\
5394 \\
\end{array}$ & $\begin{array}{c}1330.51 \\
1059 \\
\end{array}$ & $\begin{array}{c}1235.66 \\
6074 \\
\end{array}$ & $\begin{array}{c}1252.36 \\
896 \\
\end{array}$ & $\begin{array}{c}1241.22 \\
975 \\
\end{array}$ & $\begin{array}{c}1201.93 \\
0765 \\
\end{array}$ & $\begin{array}{c}1230.36 \\
7334 \\
\end{array}$ & $\begin{array}{c}1312.49 \\
7798 \\
\end{array}$ \\
\hline \multicolumn{13}{|l|}{ METADATO } \\
\hline TIPO & Punto & \multirow{2}{*}{\multicolumn{11}{|c|}{$\begin{array}{l}\text { Punto } \\
\text { vB2 }\end{array}$}} \\
\hline NAME & vB2 & & & & & & & & & & & \\
\hline $\mathrm{x}$ & $\begin{array}{c}1604.34 \\
1016\end{array}$ & $\begin{array}{c}1604.75 \\
3847\end{array}$ & $\begin{array}{c}1602.56 \\
8414\end{array}$ & $\begin{array}{c}1606.29 \\
5122\end{array}$ & $\begin{array}{c}1605.27 \\
2739 \\
\end{array}$ & $\begin{array}{c}1603.08 \\
8819\end{array}$ & $\begin{array}{c}1602.82 \\
9767\end{array}$ & $\begin{array}{c}1605.44 \\
4454\end{array}$ & $\begin{array}{c}1603.45 \\
8491\end{array}$ & $\begin{array}{c}1603.38 \\
4556\end{array}$ & $\begin{array}{c}1603.51 \\
9617\end{array}$ & $\begin{array}{c}1603.91 \\
2702\end{array}$ \\
\hline Y & $\begin{array}{c}1219.65 \\
4498 \\
\end{array}$ & $\begin{array}{c}1221.30 \\
9563\end{array}$ & $\begin{array}{c}1222.97 \\
6173\end{array}$ & $\begin{array}{c}1194.93 \\
5517\end{array}$ & $\begin{array}{c}1187.40 \\
2251 \\
\end{array}$ & $\begin{array}{c}1285.05 \\
293 \\
\end{array}$ & $\begin{array}{c}1201.88 \\
272 \\
\end{array}$ & $\begin{array}{c}1212.49 \\
5884\end{array}$ & $\begin{array}{c}1201.50 \\
0354\end{array}$ & $\begin{array}{c}1158.66 \\
3945\end{array}$ & $\begin{array}{c}1186.94 \\
3454\end{array}$ & $\begin{array}{c}1266.34 \\
1965\end{array}$ \\
\hline
\end{tabular}

Estos valores representan coordenadas (x,y) en pixels sobre la imagen, a partir de las cuales se obtiene el valor de desgaste para cada filo de corte, como se ve en el Anexo I.6. 


\subsubsection{PROCEDIMIENTO DE MEDIDA DEL DELAMINADO}

El delaminado es un defecto habitual que aparece al mecanizar piezas de material compuesto con refuerzo de tejido. Tras el mecanizado, en los laminados de material compuesto se puede observar delaminado en las caras más exteriores de la pieza, que consiste en la separación entre las distintas capas de tejido por efecto de la fuerza de corte.

En un proceso de fresado, como ya se ha descrito en el panorama científico, se han identificado tres tipos distintos de delaminado (I, II y III) [89], en torno al borde de la superficie mecanizada del laminado. Para plantear el método de medida primero se van a identificar estos tipos de delaminado en el borde fresado de la pieza (Figuras 2.20 y 2.21).

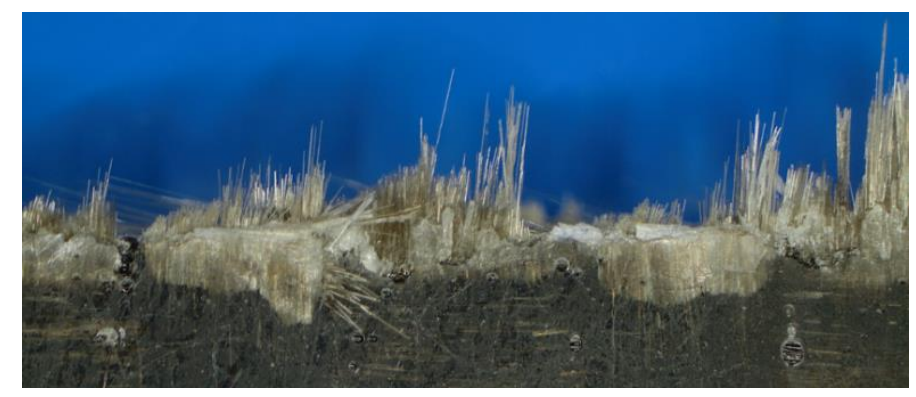

Figura 2.20. Detalle del borde fresado de una pieza con delaminado.

El delaminado tipo I (Figura 2.21, amarillo), aparece cuando las fibras de las capas exteriores, superior e inferior del tejido, se separan sobre la superficie de la pieza, siempre del borde mecanizado hacia dentro de la pieza, y se desprende la resina superficial.

El delaminado tipo II (Figura 2.21, naranja) y III (Figura 2.21, verde), aparecen cuando las fibras mal cortadas sobresalen del borde mecanizado hacia fuera de la pieza, donde no debería haber material después del mecanizado y debería quedar un corte limpio. El delaminado tipo II se produce en la dirección de la urdimbre del tejido, y el delaminado tipo III, ligeramente alineado con la trayectoria de corte.

Los delaminados tipo II y III provocan en la pieza un efecto de deshilachado del borde mecanizado y se puede definir como las fibras de refuerzo de material compuesto que la herramienta no ha sido capaz de cortar y que sobresalen sobre el borde del laminado, creando un efecto de hilos sueltos y desordenados en exceso sobre la cota dimensional establecida. 

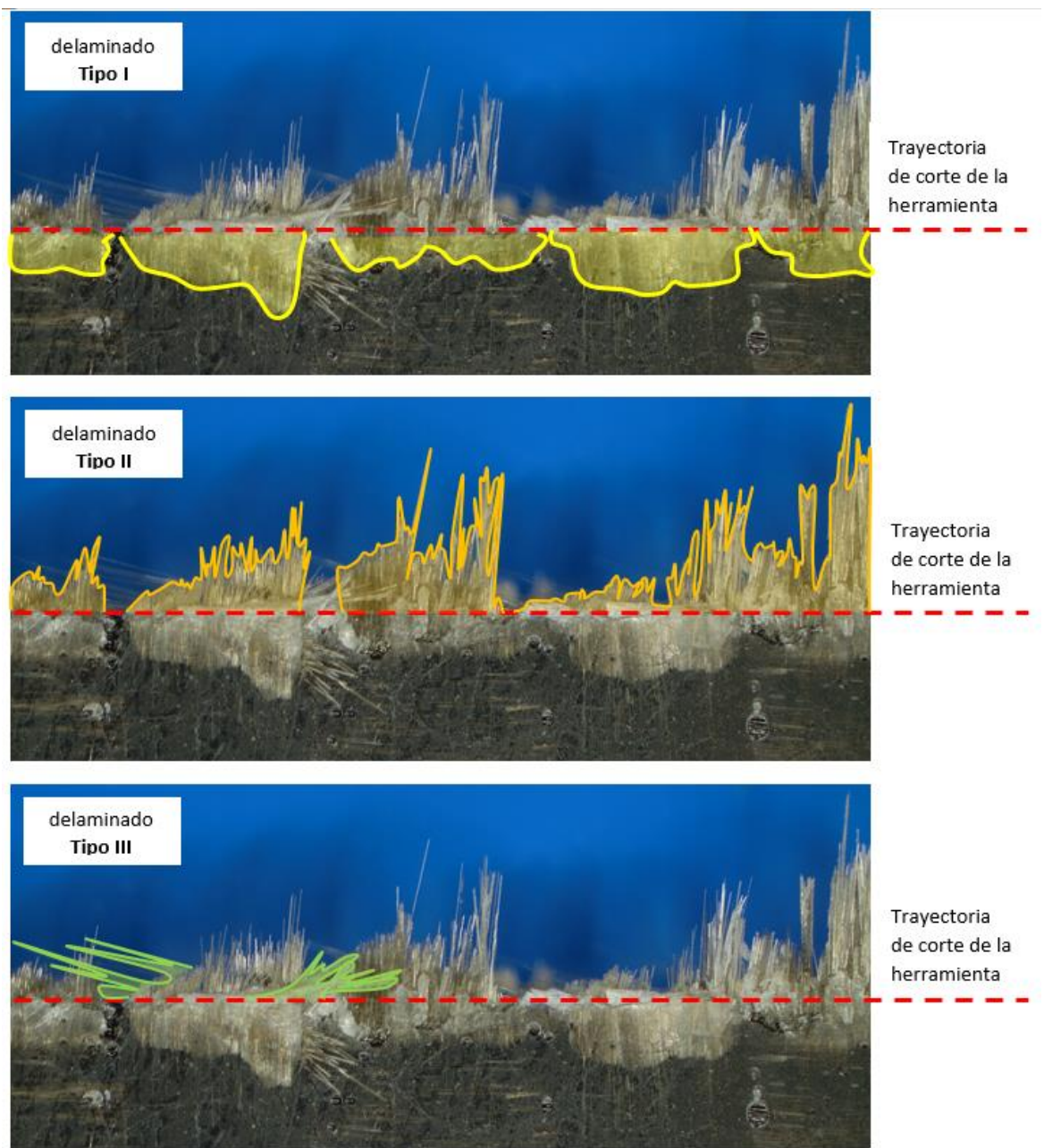

Figura 2.21. Tipos de delaminado.

Para evaluar el delaminado en una determinada longitud de pieza mecanizada (L), se han definido parámetros de medida unidimensionales y de área.

Los parámetros unidimensionales evalúan las longitudes medias o máximas de las fibras $\left(\mathrm{h}_{\max } \mathrm{i}\right)$, mientras que los de superficie cuantifican la densidad del delaminado $\left(\mathrm{Sd}_{\mathrm{i}}\right)$. Aunque estos parámetros se describirán y definirán con más detalle en el capítulo 4, aquí se van a mostrar las medidas que se han realizado para cada uno de los tipos de delaminado. 
Los parámetros que se han definido y medido para cada mecha de tejido, son los siguientes (Figura 2.22):

- $\mathrm{h}_{\max }(\mathrm{mm})$, representa la altura máxima en cada mecha “i”. En el delaminado tipo I se evalúan las mechas que van hacia dentro de la pieza y en los tipos II y III es la altura de las fibras que sobresalen de la pieza.

- Sdi $\left(\mathrm{mm}^{2}\right)$, en el delaminado tipo I representa el área dañada en cada mecha "i", sobre la superficie de la pieza, donde por efecto de la herramienta de corte se ha arrancado la resina superficial y se despegan las fibras de la capa más exterior del laminado (desconchado o daño superficial). En los tipos II y III representa la superficie de hebras sueltas, de los mechones que sobresalen de la línea de corte hacia fuera del laminado.

- li (mm), para el delaminado tipo I representa la longitud de borde del laminado donde se produce el defecto de desconchado o daño superficial en cada mecha "i", mientras que para los tipos II y III representa la longitud del borde del laminado invadida por la hebra mal cortada.

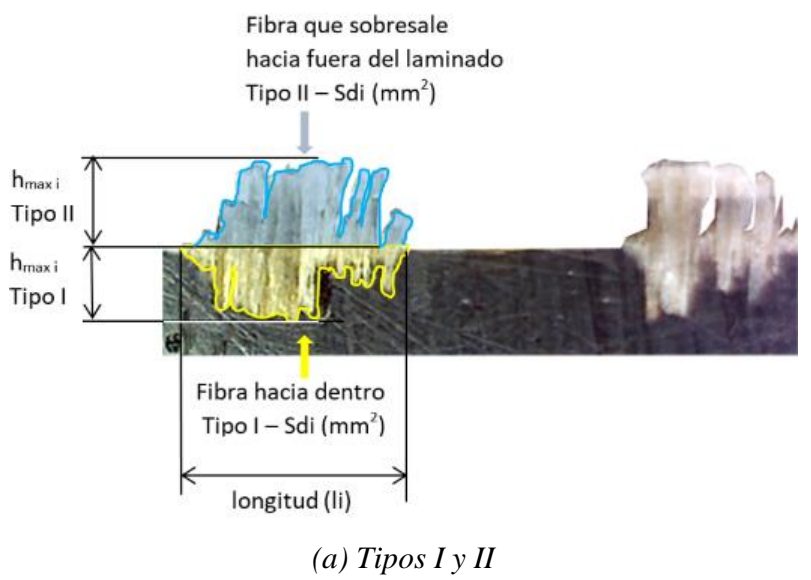

Figura 2.22. Parámetros evaluados de delaminado.

La medida del delaminado se ha realizado obteniendo imágenes del borde superior de los laminados, para todos los experimentos y en los tiempos de corte establecidos. El software libre GIMP 2.0 se ha utilizado para el análisis de las imágenes del delaminado de las piezas. Para cada mecha del tejido se captura la imagen, los parámetros $\mathrm{h}_{\max }$ i, $\mathrm{Sd}_{\mathrm{i}} \mathrm{y} \mathrm{l}_{\mathrm{i}}$ para cada tipo de delaminado. 
En las Figuras 2.23 y 2.24 se puede ver un ejemplo de cómo se ha realizado la captura para una mecha determinada (mecha 4$)$.

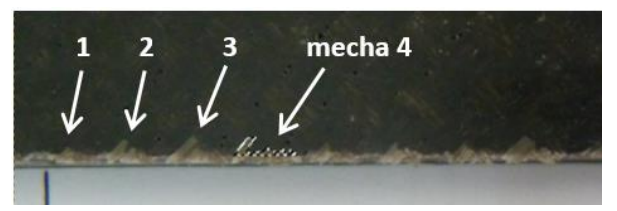

Figura 2.23. Ejemplo de numeración de mechas en la longitud evaluada

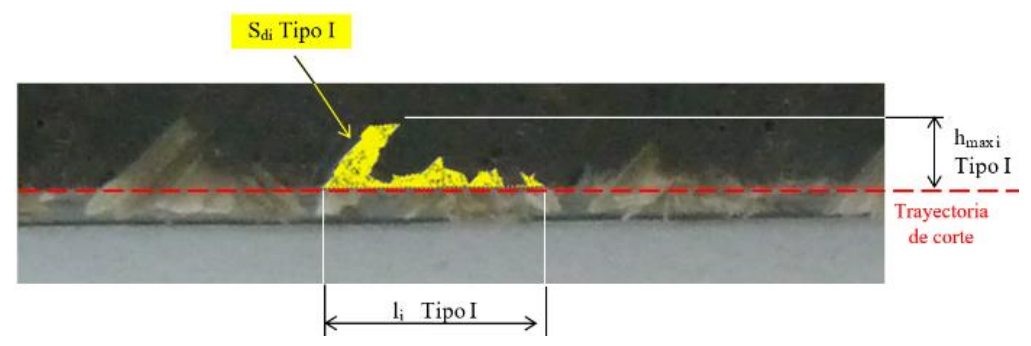

(a)

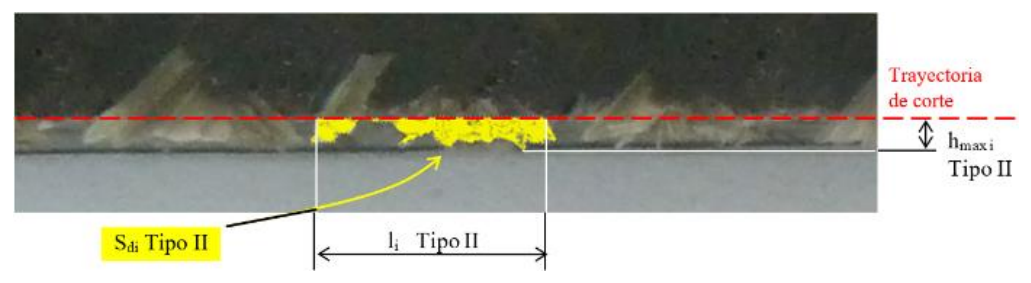

(b)

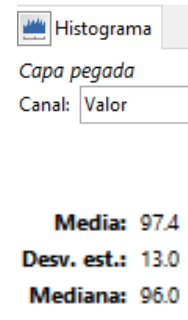

Desv. est.: 13.0

Mediana: 96.0
๑

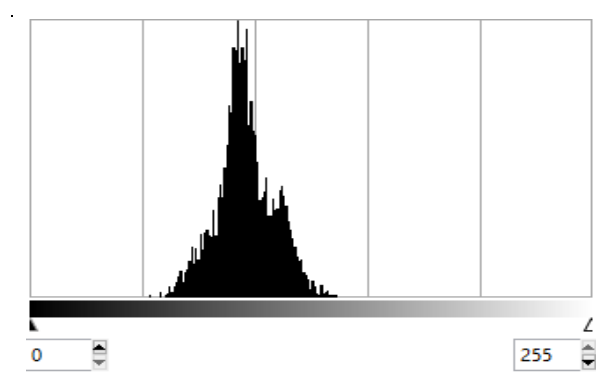

(c)

Figura 2.24. Ejemplo Mecha 4 parámetros delaminado (a) tipo I (b) tipo II (c) histograma tipo I

Las áreas $\left(\mathrm{Sd}_{\mathrm{i}}\right)$ se obtienen mecha a mecha, a partir de un recorte y un histograma donde aparece el número de pixels seleccionados de la imagen. Los valores obtenidos a partir de las imágenes se han registrado en tablas para su 
posterior análisis y cálculo de los parámetros de salida. La tabla 2.5 muestra un ejemplo de cómo se han registrado los datos medidos para uno de los experimentos.

Tabla 2.5. Ejemplo de parámetros medidos tipo II - E12

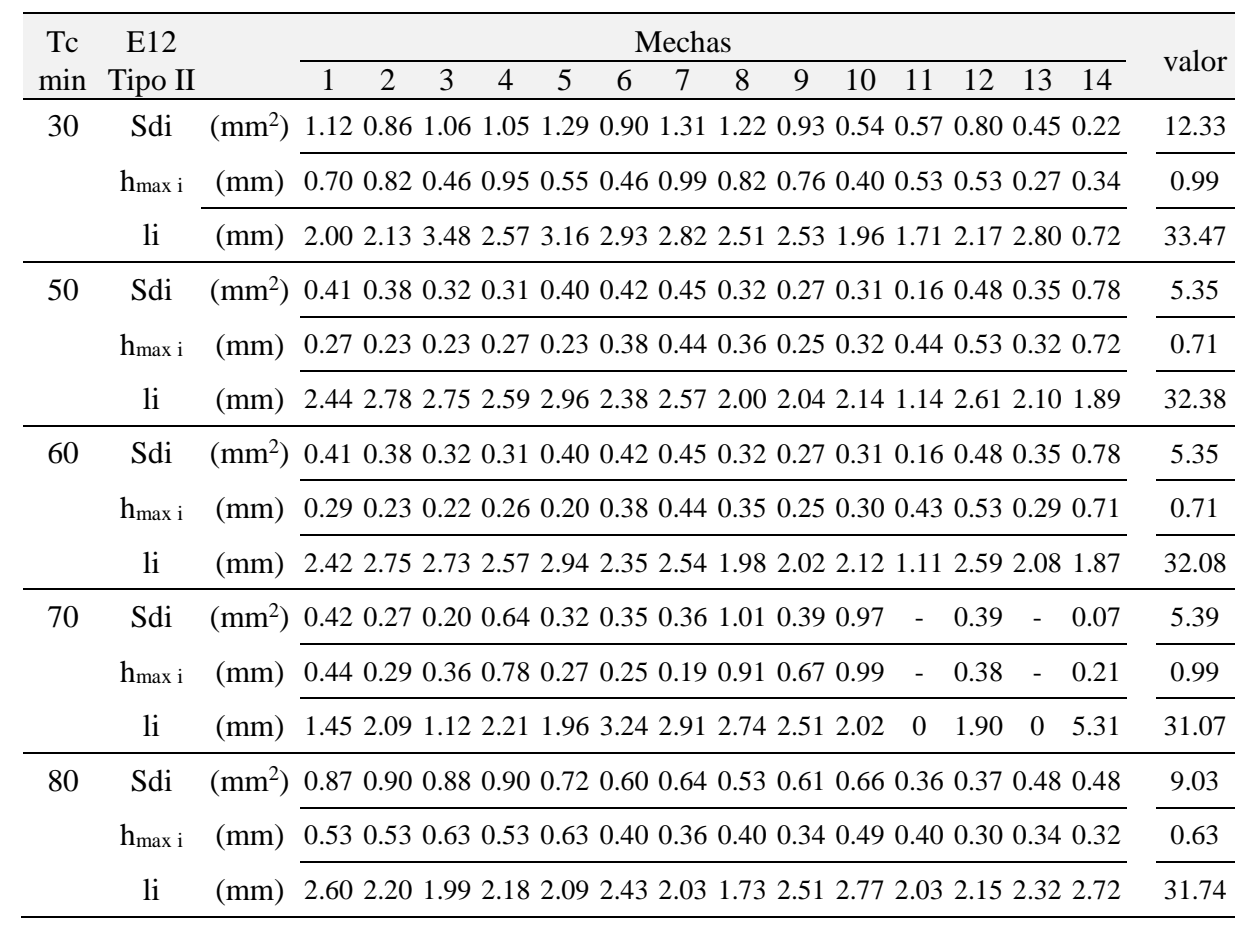

\subsection{TÉCNICAS ESTADÍSTICAS}

En esta sección se presentan las diferentes técnicas estadísticas utilizadas en el análisis de las diferentes variables incluidas en esta tesis. En primer lugar, se presenta el Análisis de la Varianza (ANOVA) que tiene por objetivo básico la comparación de las medias de más de dos poblaciones. Adicionalmente, con el objetivo de disponer de un modelo de predicción se han considerado el modelo de regresión cuantílica. Estas técnicas se describen en los siguientes apartados.

\subsubsection{ANÁLISIS DE LA VARIANZA (ANOVA)}

El Análisis de la Varianza (ANOVA) es una técnica estadística básica cuyo objetivo es comprobar la existencia o no de diferencias estadísticamente 
significativas entre las medias de conjuntos de datos. En ocasiones, el error de medición o la variabilidad asociada al proceso que se analiza hace complicado observar las diferencias existentes. En el contexto del ANOVA, la variable que define los diferentes grupos se le denomina factor mientras la variable para la que se desean comparar los diferentes grupos se le denomina independiente.

Los conjuntos de datos pueden presentar diferencias debidas a las diferentes condiciones en las que se realiza la experimentación (por ejemplo, en el caso de esta tesis, la velocidad de corte, la orientación de la fibra, etc.) de forma que los niveles de cada factor se han fijado previamente denominándose el modelo de análisis de la varianza de efectos fijos.

El ANOVA permite evaluar el efecto individual y conjunto de dos o más factores sobre una variable dependiente de tipo cuantitativo. El utilizar más de un factor tiene la ventaja de poder estudiar el efecto que la interacción entre los factores considerados tiene sobre la variable respuesta. En un ANOVA clásico con muestras independientes se plantea una hipótesis nula sobre cada factor analizado y cada posible combinación de factores. La hipótesis nula que se plantea sobre cada factor establece que las medias correspondientes a los niveles de cada factor son iguales mientras que la hipótesis nula asociada al efecto de una interacción considera que tal efecto es nulo.

Habitualmente los resultados del ANOVA se expresan en forma de tabla a partir de la descomposición de la varianza total. En la Tabla 2.6 se muestra un ejemplo de la tabla ANOVA considerando dos factores e interacción.

Tabla 2.6. Ejemplo tabla ANOVA dos factores con interacción.

\begin{tabular}{lclll}
\hline Variación & $\begin{array}{c}\text { Grados de } \\
\text { libertad }(\mathrm{gdl})\end{array}$ & $\begin{array}{c}\text { Suma de } \\
\text { cuadrados }\end{array}$ & Cuadrado Medio & $F$ \\
\hline Factor A & $J-1$ & $S C_{A}$ & $M C_{A}=S C_{B} /(J-1)$ & $M C_{A} / M C_{\text {Intra }}$ \\
\hline Factor B & $K-1$ & $S C_{B}$ & $M C_{B}=S C_{B} /(K-1)$ & $M C_{B} / M C_{\text {Intra }}$ \\
\hline Interacción & $(J-1)(K-1)$ & $S C_{A B}$ & $M C_{A B}=S C_{A B} /(J-1)(K-1)$ & $M C_{A B} / M C_{\text {Intra }}$ \\
\hline Residual & $(n-J K)$ & $S C_{\text {Intra }}$ & $M C_{\text {Intra }}=S C_{\text {Intra }} /(n-J K)$ & \\
\hline Total & $(n-1)$ & $S C T$ & & \\
\hline
\end{tabular}

En el caso del ejemplo mostrado en la Tabla 2.6 con dos factores e interacción, para cada uno de los factores y la interacción si la hipótesis nula es cierta la distribución muestral de estadístico F sigue una distribución F de Snedecor con sus correspondientes grados de libertad. 
Una vez determinado si los diferentes factores analizados son significativos se puede estimar el denominado tamaño del efecto (ecuación 2.1), que indica la magnitud de los factores que se consideren estadísticamente significativos y que se puede evaluar como:

$$
\eta^{2}=\frac{S C \text { entre }}{S C \text { total }}
$$

(ecuación 2.1)

$\eta^{2}$ toma valores entre 0 y 1 . De forma general se suele considerar que valores de $\eta^{2}$ en torno a 0.01 implican un efecto pequeño, 0.06 implican un efecto medio y valores de 0.14 un efecto grande.

La aplicación del ANOVA implica los siguientes supuestos: 1) las observaciones proceden de poblaciones normales, 2) las muestras (los grupos a comparar) son aleatorias e independientes, 3 ) dentro de cada muestra (grupo) las observaciones son independientes y 4) homocedasticidad (igualdad de varianzas) de las observaciones.

La comprobación del supuesto de normalidad se puede realizar mediante el test de Saphiro-Wilk (ecuación 2.2). Este test se basa en calcular la media y la varianza muestral. Las observaciones se ordenan de menor a mayor y se calculan las diferencias entre primero y último, segundo y penúltimo, etc. Estas diferencias se corrigen mediante unos coeficientes tabulados y se calcula el estadístico de prueba.

$$
W=\frac{D^{2}}{n \cdot s^{2}}
$$

(ecuación 2.2)

siendo $D$ la suma de las diferencias corregidas y $s^{2}$ la varianza muestral. La hipótesis nula de normalidad se rechaza si el estadístico $W$ es menor que el valor crítico proporcionado por la tabla elaborada por los autores para el tamaño muestral y el nivel de significación dado.

La comprobación del supuesto de homocedasticidad se puede realizar mediante el test de Barlett, que permite estudiar la igualdad de varianzas para dos o más grupos. Si se considera el caso particular de k poblaciones, bajo el supuesto de que son normales y tienen igual varianza $\left(H_{0}: \sigma_{1}^{2}=\sigma_{2}^{2}=\right.$ $\cdots=\sigma_{k}^{2}$ ) el estadístico de contraste, calculado a partir de las varianzas, sigue una distribución $\chi_{k-1}^{2}$. Una alternativa al test de Bartlett es el test de Levene. El test de Levene computa un estadístico basado en la diferencia absoluta de cada observación respecto a la media de su grupo. Bajo la hipótesis nula de igualdad de varianzas dicho estadístico sigue una distribución $\mathrm{F}_{\mathrm{k}-1, \mathrm{n}-\mathrm{k}}$.

El ANOVA clásico es bastante robusto ante la presencia de falta normalidad y falta de homocedasticidad si los tamaños de muestra son iguales. No 
obstante, la falta de independencia y la presencia de datos anómalos podrían invalidar las conclusiones de un ANOVA. Una alternativa para resolver los problemas derivados del incumplimiento de los supuestos en pruebas paramétricas es la aplicación de los denominados métodos robustos.

Diferentes aproximaciones se han propuesto en la literatura para la aplicación de ANOVA robustos. Una de estas aproximaciones es el uso de medias recortadas ("trimmed means"). La media recortada es un estimador robusto de la tendencia central y tiene como objetivo evitar la influencia que las observaciones extremas pueden tener sobre la media aritmética. El cálculo de la media recortada se realiza excluyendo un porcentaje determinado $(\gamma)$ de los valores en los dos extremos de la distribución y evaluando la media aritmética de los restantes valores. El uso de la media recortada presenta dos ventajas: a) proporciona una mejor estimación de la posición cuando se tiene una distribución asimétrica y b) la desviación típica de la media recortada es menos sensible a la presencia de datos atípicos y asimetría.

Así, se utilizan como estimadores de posición y dispersión la media recortada y el error estándar de dicha media. Para la realización del ANOVA se emplea la generalización robusta del test de Welch, [97] utilizado en la comparación de medias, en lugar del contraste F, si se incumple el supuesto de homocedasticidad, [98].

Las hipótesis nula y alternativa del ANOVA robusto son las mismas que en el ANOVA paramétrico utilizando las medias recortadas (ecuaciones 2.3, 2.4 y 2.5):

$$
\text { Factor A: } H_{0}: \sum_{j=1}^{J} \alpha_{j}^{2}=0
$$

(ecuación 2.3)

Factor B: $H_{0}: \sum_{k=1}^{K} \beta_{k}^{2}=0$

Interacción: $H_{0}: \sum_{k=1}^{K} \beta_{k}^{2}=0$ (ecuación 2.4)

(ecuación 2.5)

Siendo $\mu_{t}$ la gran media recortada (población), $\mu_{t j k}$ la media para una combinación de factores $j k, \mu_{t j}$. $y \mu_{t . k}$ la media recortada para el factor A y B respectivamente, $\alpha_{j}=\mu_{t j} .-\mu_{t}, \quad \beta_{k}=\mu_{t k}-\mu_{t}$ y $(\alpha \beta)_{j k}=\mu_{t j k}-\mu_{t j .}-$ $\mu_{t . k}+\mu_{t}$.

Otro de los supuestos del ANOVA, tal como se ha comentado previamente, es la independencia de las observaciones. Si la variable independiente corresponde a mediciones en diferentes instantes de tiempo en los mismos individuos no se verifica el supuesto de independencia y se requiere un ANOVA específico denominado ANOVA de medidas repetidas. La hipótesis 
nula que se establece en el ANOVA de medidas repetidas es, al igual que en los casos anteriores, la igualdad de medias poblacionales frente a la alternativa de que, al menos dos medias son distintas.

El estadístico de contraste es la razón F el cual se obtiene como el cociente entre la media cuadrática entre-grupos y la media cuadrática del error. Así, a diferencia del ANOVA de muestras independientes para un factor donde la varianza se divide en dos componentes: la varianza entre-grupos y el error, en el ANOVA de medidas repetidas con un factor, la varianza se divide en tres componentes: la varianza entre-grupos, la varianza entre-sujetos y la varianza asociada al error. En la Tabla 2.7 se muestra un ejemplo de la tabla ANOVA de un factor de medidas repetidas.

Tabla 2.7. Ejemplo tabla ANOVA de un factor de medidas repetidas.

\begin{tabular}{lcclc}
\hline Variación & $\begin{array}{c}\text { Grados de } \\
\text { libertad }(\mathrm{gdl})\end{array}$ & $\begin{array}{c}\text { Suma de } \\
\text { cuadrados }\end{array}$ & Cuadrado Medio & $F$ \\
\hline $\begin{array}{l}\text { Entre } \\
\text { grupos }\end{array}$ & $J-1$ & $S C_{F}$ & $M C_{A}=S C \quad /(J-1)$ & $M C_{A} / M C_{\text {res }}$ \\
\hline $\begin{array}{l}\text { Entre } \\
\text { sujetos }\end{array}$ & $n-1$ & $S C_{E S}$ & & \\
\hline Residual & $(\mathrm{J}-1)(\mathrm{n}-1)$ & $S C_{\text {res }}$ & $M C_{\text {res }}=S C_{\text {res }} /(\mathrm{J}-1)(\mathrm{n}-1)$ & \\
\hline Total & $(n J-1)$ & & & \\
\hline
\end{tabular}

Si la hipótesis nula es cierta la distribución muestral de estadístico F sigue una distribución F de Snedecor con (J-1), (J-1)(nJ-1) grados de libertad.

El ANOVA de medidas repetidas requiere la verificación del supuesto de esfericidad. La esfericidad implica que las variaciones de las diferencias entre todos los pares de variables que se comparan son iguales. Por ejemplo, si se desea comparar la media de una variable entre tres niveles (I, II y II) no se rechaza el supuesto de esfericidad si la varianza entre las diferencias (I-II, I-III y II-III) son iguales. El supuesto de esfericidad se puede verificar mediante la prueba de Mauchly, [99] cuyo estadístico de contraste (ecuación 2.6), se calcula como:

$$
W=\frac{\left|C^{* \prime} \sum C^{*}\right|}{\left|\operatorname{traza} \frac{C^{* \prime} \sum C^{*}}{J-1}\right|^{J-1}}
$$

donde $\sum$ es la matriz de varianza-covarianzas muestral y $C^{*}$ la matriz de 
coeficientes ortonormales de orden $(J-1) \cdot J$ y $J$ el número de niveles del factor.

Si no se cumple el supuesto de esfericidad, se pueden utilizar diferentes correcciones que modifican los grados de libertad del numerador y denominador multiplicándolos por un factor corrector como la corrección de Greenhouse-Geisser y la de Huynh-Feld, [100].

\subsubsection{MODELO DE REGRESIÓN LINEAL MÚLTIPLE}

El modelo de regresión lineal múltiple es uno de los modelos de regresión ampliamente utilizado en la literatura que permite el análisis de la relación entre una variable dependiente $(Y)$ y un conjunto de variables explicativas $\left(X_{i}\right)$. El modelo de regresión lineal múltiple se expresa como:

$$
E\left(Y_{i}\right)=\mu_{i}=X_{i}^{T} \cdot \beta+u \quad \text { (ecuación 2.7) }
$$

donde $E$ representa el valor medio o esperado, $X_{i}^{T}=\left(1, X_{1}, X_{2}, \ldots, X_{p}\right), \beta^{T}=$ $\left(\beta_{0}, \beta_{1}, \ldots, \beta_{\mathrm{p}}\right), p$ es el número de variables independientes y $u=N(0, \sigma)$ es el error aleatorio. La estimación de los coeficientes $\beta_{i}$ en el modelo de regresión lineal múltiple se realiza mediante Mínimos Cuadrados Ordinarios (MCO).

Un aspecto a considerar en los modelos de regresión es la selección del mejor conjunto de variables. En la literatura se han propuesto diferentes procedimientos de selección de variables entre los que encuentran los métodos Stepwise. Este procedimiento es una combinación de los métodos forward y backward. En los métodos forward se parte del modelo más sencillo y se van añadiendo variables independientes en función de un criterio previamente definido hasta que se cumple una regla de finalización del algoritmo. Por el contrario, el enfoque de los métodos backward se basa en comenzar con un modelo muy complejo, que incluye todas las variables independientes consideradas inicialmente, e ir eliminado de forma progresiva las variables menos influyentes hasta que se verifica un criterio de parada. El método stepwise combina los dos métodos anteriores de forma que va introduciendo progresivamente variables independientes, pero en cada etapa se plantea si las variables que han sido introducidas deben permanecer o no en el modelo.

El criterio para la introducción o eliminación de variables independientes se puede basar en la significación de forma que, en el método forward, se añade al modelo la variable que resulta más significativa mientras que, en el método backward, se eliminaría la variable que resulte menos significativa de todas las incluidas en el modelo de regresión. Otros criterios que pueden ser utilizados para incluir o eliminar variables independientes del modelo de regresión son el criterio de información de Akaike (AIC) y el Criterio de Información 
Bayesiano (BIC) siendo el objetivo, en este caso, buscar el modelo con el AIC o BIC más pequeño.

Los supuestos básicos del modelo de regresión lineal son: ausencia de multicolinealidad, linealidad, homocedasticidad, normalidad e independencia de los errores.

Si las variables explicativas pueden ser expresadas como una combinación lineal se tiene un problema de multicolinealidad. El problema de multicolinealidad se puede resolver eliminado las variables independientes que presentan dependencia. Una de las ventajas que presentan los métodos de selección de variables descritos anteriormente es que solucionan el problema de multicolinealidad.

Los supuestos de normalidad, homocedasticidad e independencia pueden ser contrastados a partir de los residuos. El residuo $u_{i}$ se define como:

$$
u_{i}=\mathrm{y}_{i}-\hat{\mathrm{y}}_{i} \quad \text { (ecuación 2.8) }
$$

siendo $\hat{y}_{i}$ el valor estimado de la variable dependiente a partir del modelo de regresión ajustado.

El supuesto de normalidad de los residuos se puede verificar gráficamente, a partir de un histograma de los residuos o el gráfico de probabilidad normal, o mediante el test de Saphiro Wilks descrito anteriormente.

La homocedasticidad supone que la variabilidad observada en los residuos debe ser constante en todo el rango de observaciones. Este supuesto se puede verificar analizando el gráfico de los valores estimados por el modelo frente a los residuos al cuadrado de forma que, si la variabilidad es constante, se observara la misma dispersión y una distribución aleatoria sin presencia de patrones de comportamiento. Una alternativa es la aplicación del test de razón de varianzas.

Finalmente, la independencia puede ser contrastada gráficamente a partir del comportamiento de los residuos comportamientos no aleatorios de los mismos pueden implicar la presencia de autocorrelación.

\subsubsection{MODELO DE REGRESIÓN CUANTÍLICA}

La regresión cuantílica según Koenker y Basset [101], es un método de estimación de la relación entre un conjunto de variables predictoras y cuantiles específicos de la variable respuesta alternativo a los métodos clásicos de regresión por MCO. 
El poder realizar la regresión sobre cualquier cuantil permite conocer cómo influyen las diferentes variables independientes (predictores) en todo el rango de la variable respuesta.

Por otro lado, mientras la regresión mediante MCO requiere de unos supuestos previos sobre el comportamiento sobre el comportamiento de los residuos que han sido presentados en la sección anterior la regresión cuantílica no considera ninguna restricción sobre error. Esta relajación de restricciones respecto al modelo de regresión lineal clásico permite aplicar la regresión cuantílica en ausencia de normalidad, dado que no realiza ningún supuesto sobre la distribución de la variable dependiente, y de homocedasticidad (igualdad de varianza). Finalmente, otra ventaja que presenta este modelo es un mejor ajuste en presencia de datos atípicos.

Un cuantil $q$ se puede definir como el valor que minimiza una suma ponderada, ponderando más la parte con menos observaciones (ecuación 2.9). La mediana constituye un caso especial siendo $q=0.5$ y teniendo todas las observaciones igual ponderación:

$$
\min (b \in R)\left[\sum_{Y_{i} \geq b} q\left|Y_{i}-b\right|+\sum_{Y_{i}<b}(1-q)\left|Y_{i}-b\right| \quad\right. \text { (ecuación 2.9) }
$$

De forma similar se puede plantear los cuantiles de $Y$ condicionados a los valores de $X$, pudiendo definir los cuantiles de $\beta x_{i}$ (ecuación 2.10) de forma análoga a la ecuación (2.9) anterior como:

$$
\min (\beta \in R)\left[\sum_{Y_{i} \geq b} q\left|Y_{i}-\beta x_{i}\right|+\sum_{Y_{i}<b}(1-q)\left|Y_{i}-\beta x_{i}\right| \quad\right. \text { (ecuación 2.10) }
$$

La obtención de los parámetros que minimizan la ecuación (2.10) son una estimación cuantílica. La solución al problema de minimización planteado se realiza habitualmente mediante programación lineal o por métodos de optimización iterativos. 



\section{CAPÍTULO 3. ANÁLISIS DE LA RUGOSIDAD SUPERFICIAL Y EL DESGASTE DE LA HERRAMIENTA}

\subsection{INTRODUCCIÓN}

En este capítulo se van a analizar tanto la rugosidad superficial (Ra) del laminado como el desgaste que sufre la herramienta en diferentes tiempos de mecanizado.

La rugosidad de la superficie mecanizada es uno de los parámetros estudiados en el mecanizado de materiales compuestos porque se usa como indicador de la calidad del mecanizado. En relación al desgaste de la herramienta $(\mathrm{Vb})$ se pretende evaluar la pérdida de la capacidad de corte que sufre la herramienta con el objetivo de cuantificar el tiempo de vida de la herramienta seleccionada en el mecanizado de materiales compuestos con fibra de basalto.

Una vez realizados los ensayos propuestos en el diseño de experimentos, se realizará un análisis estadístico para ver la influencia de los diferentes parámetros tanto en la rugosidad como en el desgaste de la herramienta.

Finalmente, se propone un modelo para predecir la rugosidad $(\mathrm{Ra})$ en función del desgaste $(\mathrm{Vb})$ que presenta la herramienta. 


\subsection{ANÁLISIS DE LA RUGOSIDAD SUPERFICIAL}

Los valores de rugosidad media aritmética del perfil (Ra) obtenidos en cada experimento, para los distintos tiempos de corte se presentan en la Tabla 3.1. Para cada experimento y tiempo de corte, se han tomado cinco medidas en tres posiciones distintas del borde mecanizado. Para simplificar las tablas de datos, en la Tabla 3.1 se presenta el valor medio de los quince puntos, aunque todas las medidas realizadas en cada experimento se incluyen en el Anexo II.1.

La rugosidad superficial en materiales compuestos con fibra no es fácil de medir, ya que los valores obtenidos son el resultado de medir conjuntamente zonas de resina y zonas de fibra de basalto fracturadas durante el proceso de mecanizado. Por ello, estos valores presentan cierta dispersión. El efecto se acrecienta con tiempos de mecanizado mayores, ya que la herramienta presenta un mayor desgaste y las fibras se hacen más patentes en las secciones medidas.

Todos los experimentos se han completado hasta un tiempo de mecanizado de 80 minutos, con sus correspondientes paradas de toma de datos $(5,30,50$, 60,70 y 80 minutos), excepto el experimento 14. Este experimento se interrumpió por un delaminado excesivo de las piezas desde los primeros minutos de mecanizado, al mismo tiempo que se produjo un desgaste bastante rápido de la herramienta. Por ello, no se dispone de datos de rugosidad superficial para ese experimento a partir de 40 minutos de tiempo de mecanizado.

En los experimentos realizados, se aprecia que la rugosidad aumenta siempre con el tiempo de corte [102].

Tabla 3.1. Rugosidad media (Ra) para distintos tiempos de corte.

\begin{tabular}{|c|c|c|c|c|c|c|c|c|c|c|c|}
\hline \multirow[t]{2}{*}{ Exp } & \multirow{2}{*}{\multicolumn{2}{|c|}{$\begin{array}{c}\mathrm{Vc} \quad \mathrm{Fz} \\
\mathrm{m} / \mathrm{min} \\
\mathrm{mm} / \text { diente }\end{array}$}} & \multirow{2}{*}{$\begin{array}{l}\mathrm{ap}_{\mathrm{p}} \\
\mathrm{mm}\end{array}$} & \multirow{2}{*}{$\begin{array}{l}\mathrm{Fv} \\
\%\end{array}$} & \multirow{2}{*}{$\begin{array}{l}\text { Fo } \\
\text { o }\end{array}$} & \multicolumn{6}{|c|}{$\mathrm{Ra}(\mu \mathrm{m})$} \\
\hline & & & & & & $5 \mathrm{~min}$ & $30 \mathrm{~min}$ & $50 \mathrm{~min}$ & $60 \mathrm{~min}$ & $70 \mathrm{~min}$ & $80 \mathrm{~min}$ \\
\hline 01 & 470 & 0.1 & 1.5 & 40 & 90 & 2.65 & 2.81 & 3.51 & 3.82 & 3.91 & 4.23 \\
\hline 02 & 300 & 0.1 & 1.5 & 40 & 45 & 3.64 & 3.86 & 4.42 & 5.53 & 5.70 & 6.27 \\
\hline 03 & 300 & 0.4 & 0.5 & 40 & 45 & 2.90 & 2.93 & 3.83 & 4.33 & 4.38 & 4.45 \\
\hline 04 & 300 & 0.4 & 1.5 & 60 & 45 & 5.10 & 5.82 & 6.35 & 6.94 & 7.13 & 7.99 \\
\hline 05 & 470 & 0.1 & 0.5 & 60 & 90 & 2.43 & 2.74 & 2.64 & 2.92 & 3.30 & 3.34 \\
\hline 06 & 470 & 0.4 & 0.5 & 40 & 90 & 3.41 & 3.75 & 3.79 & 3.77 & 3.96 & 4.05 \\
\hline 07 & 300 & 0.1 & 0.5 & 60 & 45 & 4.51 & 5.31 & 5.57 & 6.14 & 6.75 & 7.02 \\
\hline 08 & 470 & 0.4 & 0.5 & 60 & 45 & 6.02 & 6.76 & 6.92 & 7.79 & 8.64 & 8.76 \\
\hline 09 & 470 & 0.1 & 0.5 & 40 & 45 & 3.29 & 3.73 & 3.80 & 4.12 & 4.16 & 4.40 \\
\hline 10 & 300 & 0.1 & 0.5 & 40 & 90 & 1.96 & 2.59 & 3.11 & 3.44 & 3.61 & 4.50 \\
\hline
\end{tabular}




\begin{tabular}{|c|c|c|c|c|c|c|c|c|c|c|c|}
\hline \multirow[t]{2}{*}{ Exp } & \multirow{2}{*}{$\begin{array}{c}\mathrm{Vc} \\
\mathrm{m} / \mathrm{min}\end{array}$} & \multirow{2}{*}{$\begin{array}{c}\mathrm{Fz} \\
\mathrm{m} / \mathrm{diente}\end{array}$} & \multirow{2}{*}{$\begin{array}{c}\mathrm{ap}_{\mathrm{p}} \\
\mathrm{mm}\end{array}$} & \multirow{2}{*}{$\begin{array}{l}\text { Fv } \\
\%\end{array}$} & \multirow{2}{*}{$\begin{array}{c}\text { Fo } \\
\text { o }\end{array}$} & \multicolumn{6}{|c|}{$\mathrm{Ra}(\mu \mathrm{m})$} \\
\hline & & & & & & $5 \mathrm{~min}$ & $30 \mathrm{~min}$ & $50 \mathrm{~min}$ & $60 \mathrm{~min}$ & $70 \mathrm{~min}$ & $80 \mathrm{~min}$ \\
\hline 11 & 300 & 0.1 & 1.5 & 60 & 90 & 2.56 & 3.24 & 3.58 & 3.65 & 3.79 & 4.08 \\
\hline 12 & 300 & 0.4 & 0.5 & 60 & 90 & 2.87 & 2.92 & 2.98 & 3.27 & 3.34 & 3.60 \\
\hline 13 & 470 & 0.4 & 1.5 & 40 & 45 & 5.38 & 5.82 & 6.29 & 6.33 & 6.99 & 7.05 \\
\hline 14 & 470 & 0.4 & 1.5 & 60 & 90 & 4.46 & 4.94 & - & - & - & - \\
\hline 15 & 300 & 0.4 & 1.5 & 40 & 90 & 3.16 & 3.22 & 3.49 & 3.53 & 3.66 & 3.88 \\
\hline 16 & 470 & 0.1 & 1.5 & 60 & 45 & 5.52 & 6.07 & 6.20 & 6.50 & 6.65 & 7.00 \\
\hline
\end{tabular}

En las Figuras 3.1 y 3.2 se han representado gráficamente los datos de rugosidad media aritmética (Ra) obtenida en cada tiempo de corte, para piezas con orientación de fibra $\mathrm{Fo}=0^{\circ}-90^{\circ}$ y $\mathrm{Fo}=45^{\circ}-135^{\circ}$ respectivamente, para una mejor observación de los resultados.

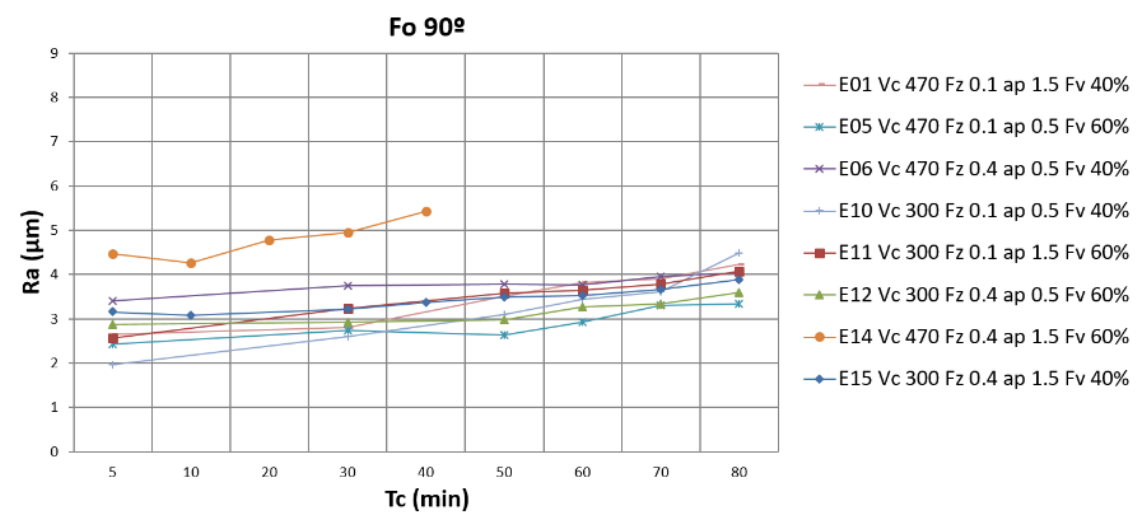

Figura 3.1. Valores de Ra con $F o=0^{\circ}-90^{\circ}$.

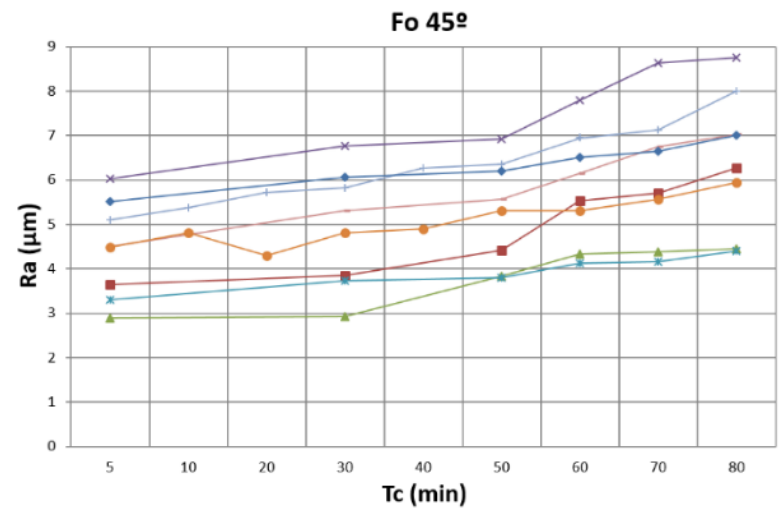

-E02 Vc $300 \mathrm{Fz} 0.1$ ap $1.5 \mathrm{Fv} 40 \%$

^-E03 Vc $300 \mathrm{Fz} 0.4$ ap $0.5 \mathrm{Fv} 40 \%$

- E04 Vc 300 Fz 0.4 ap 1.5 Fv 60\%

-E07 Vc 300 Fz 0.1 ap 0.5 Fv 60\%

* E08 Vc $470 \mathrm{Fz} 0.4$ ap $0.5 \mathrm{Fv} 60 \%$

*-E09 Vc $470 \mathrm{Fz} 0.1$ ap $0.5 \mathrm{Fv} 40 \%$

- E13 Vc4 70 Fz 0.4 ap 1.5 Fv 40\%

$\rightarrow$ E16 Vc 470 Fz 0.1 ap 1.5 Fv 60\%

Figura 3.2. Valores de Ra con Fo $=45^{\circ}-135^{\circ}$. 


\subsubsection{ANÁLISIS ESTADÍSTICO DE LA RUGOSIDAD}

En esta sección se exponen los resultados de la aplicación (al análisis de la rugosidad superficial) de las diferentes técnicas estadísticas presentadas en el capítulo 2.

\subsubsection{ANOVA multifactorial}

El objetivo de este apartado se centra en analizar si existen diferencias estadísticamente significativas en las medias de la rugosidad considerando como factores las condiciones de mecanizado ( $\mathrm{Vc}, \mathrm{Fz} \mathrm{y} \mathrm{a}_{\mathrm{p}}$ ) y el volumen ( $\mathrm{Fv}$ ) y la orientación de la fibra (Fo). En primer lugar, puesto que se han realizado mediciones en diferentes instantes de tiempo sobre el mismo laminado no se cumple la condición de independencia, por lo que se ha realizado un ANOVA de medidas repetidas con la finalidad de analizar si el tiempo de corte es un factor significativo. Un supuesto del ANOVA de medidas repetidas es que la varianza de las diferencias entre cada par de niveles debe ser la misma. Para comprobar este supuesto se ha realizado el test de Mauchly`s cuyos resultados se muestran en la Tabla 3.2 observándose un incumplimiento del supuesto de esfericidad ( $\mathrm{p}$-valor $<0.05$ ) por lo que no se realiza el test clásico y se aplican las pruebas con corrección de esfericidad de Greenhouse-Geisser (GG) y Huynh-Feldt (HF) cuyos resultados se muestran en la Tabla 3.3.

Tabla 3.2. Test de esfericidad de Mauchly.

\begin{tabular}{lcc}
\hline Efecto & W & p-valor \\
\hline Tiempo de corte $(\mathrm{Tc})$ & 0.264 & $1.74 \mathrm{E}-4$ \\
\hline
\end{tabular}

Tabla 3.3. Resultados ANOVA de medidas repetidas con corrección de esfericidad.

\begin{tabular}{lcccc}
\hline Efecto & GGe & p-valor $(\mathrm{GG})$ & HFe & p-valor $(\mathrm{HF})$ \\
\hline Tiempo de corte $(\mathrm{Tc})$ & 0.576 & $3.652 \mathrm{E}-7$ & 0.594 & $2.48 \mathrm{E}-07$ \\
\hline
\end{tabular}

El análisis ANOVA de medidas repetidas, incluyendo la corrección de esfericidad, permite concluir que existen diferencias significativas en la rugosidad media entre al menos dos de los tiempos de corte. Por ello, se van a analizar los resultados en tres tiempos de corte, al inicio del experimento $(\mathrm{Tc}=5$ $\mathrm{min}$ ), al final $(\mathrm{Tc}=80 \mathrm{~min})$ y en un tiempo intermedio $(\mathrm{Tc}=50 \mathrm{~min})$. En la Figura 3.3 se muestra el diagrama Box-Whisker múltiple correspondiente a los tres tiempos de corte analizados. 


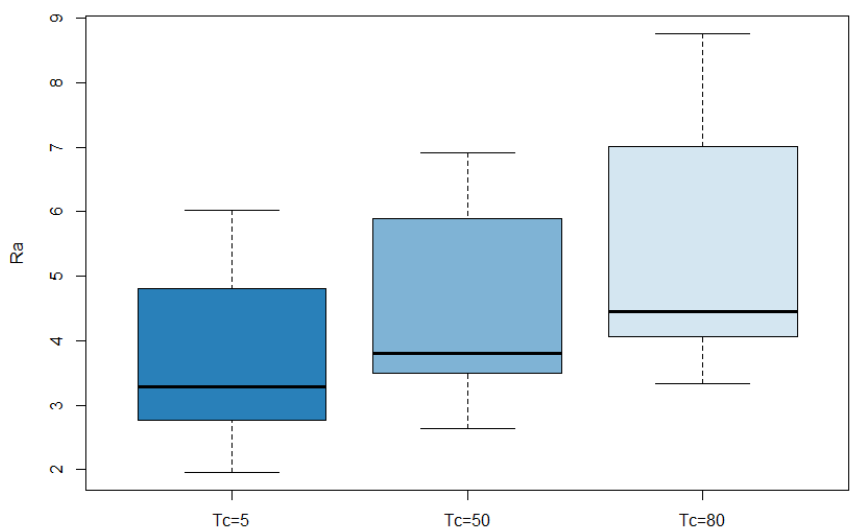

Figura 3.3. Diagrama Box-Whisker para Ra y los diferentes Tc.

Puesto que las medidas presentan una dependencia temporal, se ha realizado un ANOVA para estos instantes de tiempo $\mathrm{Tc}=5,50$ y 80 min cuyos resultados se muestran en las Tablas 3.4, 3.5 y 3.6, respectivamente. En dichas tablas se ha incorporado el tamaño del efecto $\left(\eta^{2}\right)$.

Tabla 3.4. Tabla ANOVA para Ra para Tc=5 minutos.

\begin{tabular}{|c|c|c|c|c|c|c|c|}
\hline Fuente & $\begin{array}{l}\text { Suma de } \\
\text { cuadrados }\end{array}$ & gdl & $\begin{array}{c}\text { Cuadrado } \\
\text { Medio }\end{array}$ & F-ratio & p-valor & $\eta^{2}$ & $\eta^{2}$ Parcial \\
\hline $\mathrm{Fz}$ & 2.84 & 1 & 2.84 & 9.24 & $0.012^{*}$ & 0.120 & 0.480 \\
\hline $\mathrm{Vc}$ & 2.61 & 1 & 2.61 & 8.51 & $0.015^{*}$ & 0.111 & 0.459 \\
\hline$a_{p}$ & 1.61 & 1 & 1.61 & 5.26 & $0.045^{*}$ & 0.068 & 0.344 \\
\hline Fo & 10.31 & 1 & 10.31 & 33.60 & $<0.001^{* * *}$ & 0.437 & 0.771 \\
\hline $\mathrm{Fv}$ & 3.14 & 1 & 3.13 & 10.21 & $0.010^{*}$ & 0.133 & 0.505 \\
\hline Residuos & 3.07 & 10 & 0.307 & & & & \\
\hline
\end{tabular}

Tabla 3.5. Tabla ANOVA para Ra para Tc=50 minutos.

\begin{tabular}{cccccccc}
\hline Fuente & $\begin{array}{c}\text { Suma de } \\
\text { cuadrados }\end{array}$ & gdl & $\begin{array}{c}\text { Cuadrado } \\
\text { Medio }\end{array}$ & F-ratio & p-valor & $\eta^{2}$ & $\eta^{2}$ Parcial \\
\hline Fz & 2.55 & 1 & 2.55 & 4.79 & 0.053 & 0.086 & 0.324 \\
$\mathrm{Vc}$ & 1.82 & 1 & 1.82 & 3.41 & 0.094 & 0.061 & 0.254 \\
$\mathrm{a}_{\mathrm{p}}$ & 2.86 & 1 & 2.86 & 5.38 & $0.043^{*}$ & 0.096 & 0.349 \\
$\mathrm{Fo}$ & 13.52 & 1 & 13.52 & 25.40 & $<0.001^{* * * *}$ & 0.456 & 0.718 \\
\hline
\end{tabular}




\begin{tabular}{|c|c|c|c|c|c|c|c|}
\hline Fuente & $\begin{array}{c}\text { Suma de } \\
\text { cuadrados }\end{array}$ & gdl & $\begin{array}{c}\text { Cuadrado } \\
\text { Medio }\end{array}$ & F-ratio & p-valor & $\eta^{2}$ & $\eta^{2}$ Parcial \\
\hline $\mathrm{Fv}$ & 3.58 & 1 & 3.58 & 6.73 & $0.027^{*}$ & 0.121 & 0.4022 \\
\hline Residuos & 5.32 & 10 & 0.53 & & & & \\
\hline
\end{tabular}

Tabla 3.6. Tabla ANOVA para Ra para Tc=80 minutos.

\begin{tabular}{|c|c|c|c|c|c|c|c|}
\hline Fuente & $\begin{array}{c}\text { Suma de } \\
\text { cuadrados }\end{array}$ & gdl & $\begin{array}{c}\text { Cuadrado } \\
\text { Medio }\end{array}$ & F-ratio & p-valor & $\eta^{2}$ & $\eta^{2}$ Parcial \\
\hline $\mathrm{Fz}$ & 2.20 & 1 & 2.20 & 1.71 & 0.220 & 0.047 & 0.146 \\
\hline $\mathrm{Vc}$ & 1.02 & 1 & 1.02 & 0.79 & 0.395 & 0.021 & 0.073 \\
\hline$a_{p}$ & 3.40 & 1 & 3.40 & 2.64 & 0.244 & 0.073 & 0.209 \\
\hline Fo & 20.84 & 1 & 20.84 & 16.15 & $0.002^{* * * *}$ & 0.447 & 0.618 \\
\hline $\mathrm{Fv}$ & 6.20 & 1 & 6.20 & 4.81 & 0.053 . & 0.133 & 0.325 \\
\hline Residuos & 12.91 & 10 & 1.29 & & & & \\
\hline
\end{tabular}

Las conclusiones obtenidas del ANOVA paramétrico son válidas si se verifican los supuestos de normalidad y homocedasticidad, tal y como se ha comentado en el Capítulo 2. Para verificar estos supuestos se han realizado las pruebas de Saphiro Wilks y de Barlett. Los resultados de la prueba de Saphiro Wilks para la comprobación del supuesto de normalidad se muestran en la Tabla 3.7, donde se observa que no se puede rechazar la hipótesis nula de normalidad con nivel de significación de 0.05 . Los resultados de la prueba de Barlett se muestran en la Tabla 3.8.

Tabla 3.7. Resultados de la prueba Saphiro-Wilks.

\begin{tabular}{ccc}
\hline Tc (min) & Estadístico & p-valor \\
\hline 5 & 0.970 & 0.854 \\
50 & 0.914 & 0.156 \\
80 & 0.950 & 0.559 \\
\hline
\end{tabular}

Tabla 3.8. Resultados prueba Barlett.

\begin{tabular}{ccccccc}
\hline & \multicolumn{2}{c}{$\mathrm{Tc}=5 \mathrm{~min}$} & \multicolumn{2}{c}{$\mathrm{Tc}=50 \mathrm{~min}$} & \multicolumn{2}{c}{$\mathrm{Tc}=80 \mathrm{~min}$} \\
\hline Factor & $\mathrm{K}^{2}$ & $\mathrm{p}$-valor & $\mathrm{K}^{2}$ & $\mathrm{p}$-valor & $\mathrm{K}^{2}$ & $\mathrm{p}$-valor \\
\hline $\mathrm{Fz}$ & 0.068 & 0.794 & 0.503 & 0.475 & 1.036 & 0.309 \\
$\mathrm{Vc}$ & 0.796 & 0.372 & 0.654 & 0.419 & 0.301 & 0.597 \\
$\mathrm{a}_{\mathrm{p}}$ & $4.31 \mathrm{E}-03$ & 0.983 & 0.011 & 0.916 & 0.102 & 0.749 \\
\hline
\end{tabular}




\begin{tabular}{ccccccc}
\hline & \multicolumn{2}{c}{$\mathrm{Tc}=5 \mathrm{~min}$} & \multicolumn{2}{c}{$\mathrm{Tc}=50 \mathrm{~min}$} & \multicolumn{2}{c}{$\mathrm{Tc}=80 \mathrm{~min}$} \\
\hline Factor & $\mathrm{K}^{2}$ & $\mathrm{p}$-valor & $\mathrm{K}^{2}$ & $\mathrm{p}$-valor & $\mathrm{K}^{2}$ & $\mathrm{p}$-valor \\
\hline $\mathrm{FV}$ & 1.036 & 0.309 & 2.005 & 0.157 & 5.363 & $0.003^{*}$ \\
Fo & 3.924 & $0.047^{*}$ & 6.056 & $0.014^{*}$ & 2.671 & 0.110 \\
\hline
\end{tabular}

$*$ p-valor $<0.05$

Analizando los resultados de la Tabla 3.8 se observa el incumplimiento del supuesto de homocedasticidad ( $\mathrm{p}$-valor < 0.05). La misma conclusión se obtiene si se analizan los residuos de los modelos como se observa en las Figuras 3.4, 3.5 y 3.6, para los tiempos de corte de 5, 50 y 80 minutos. En dichas figuras se puede observar en el gráfico de los residuos en función de los valores ajustados la falta de homocedasticidad, mientras el cumplimiento del supuesto de normalidad se observa en el gráfico cuantil-cuantil (normal Q-Q).
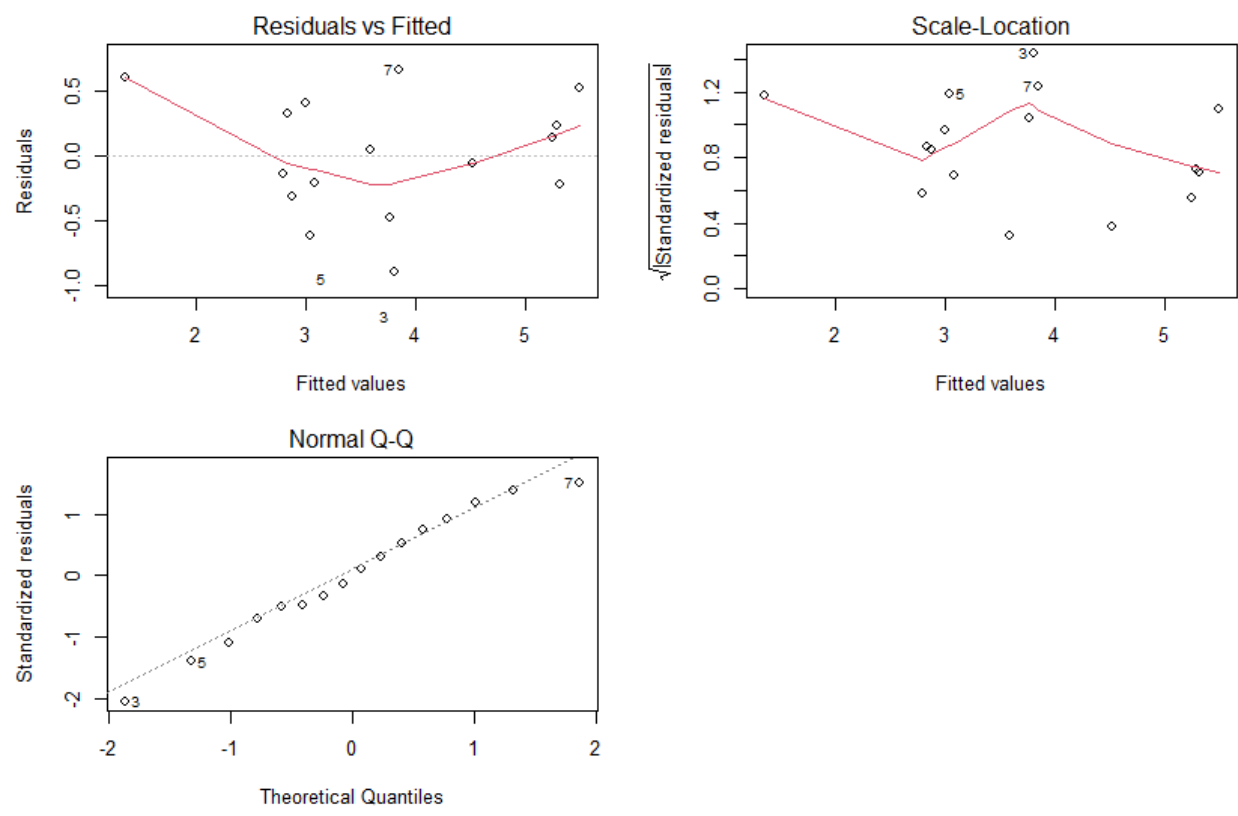

Figura 3.4. Gráficos de análisis de residuos modelo ANOVA Tc=5 minutos. 

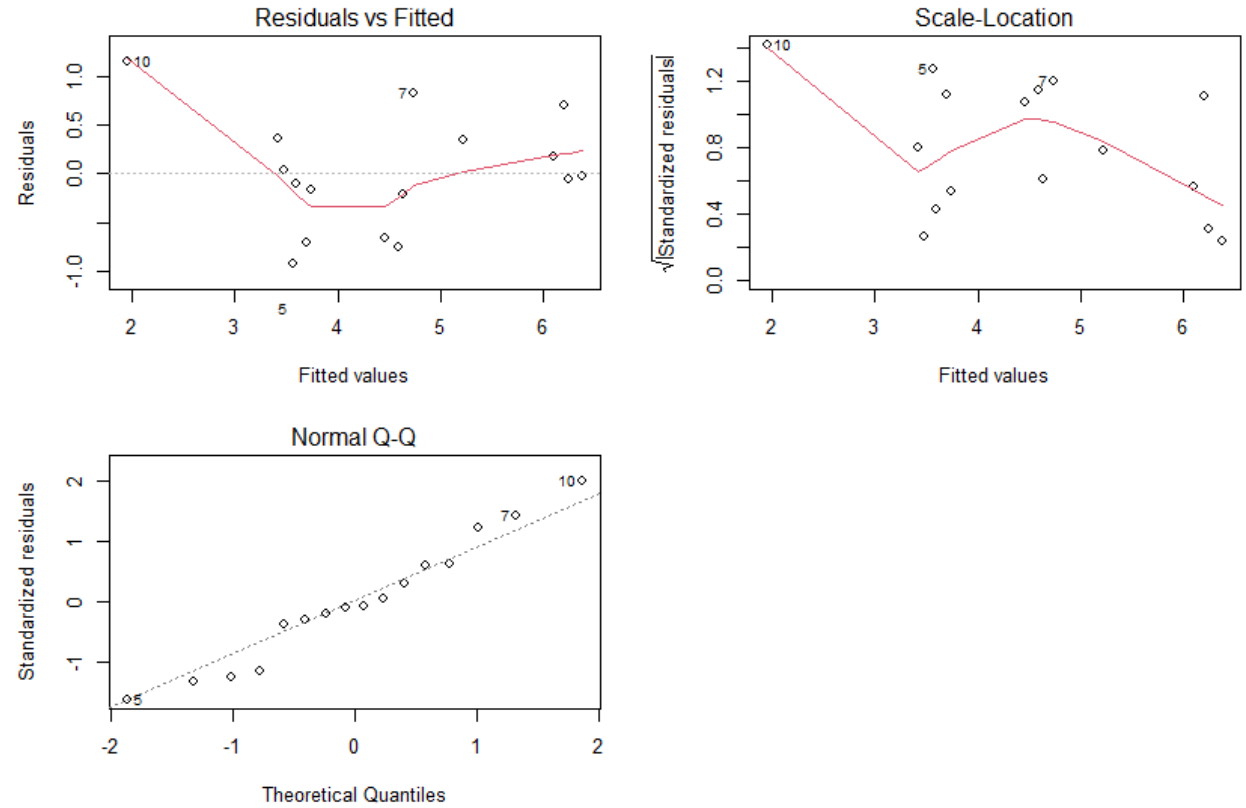

Figura 3.5. Gráficos de análisis de residuos modelo ANOVA Tc=50 minutos.
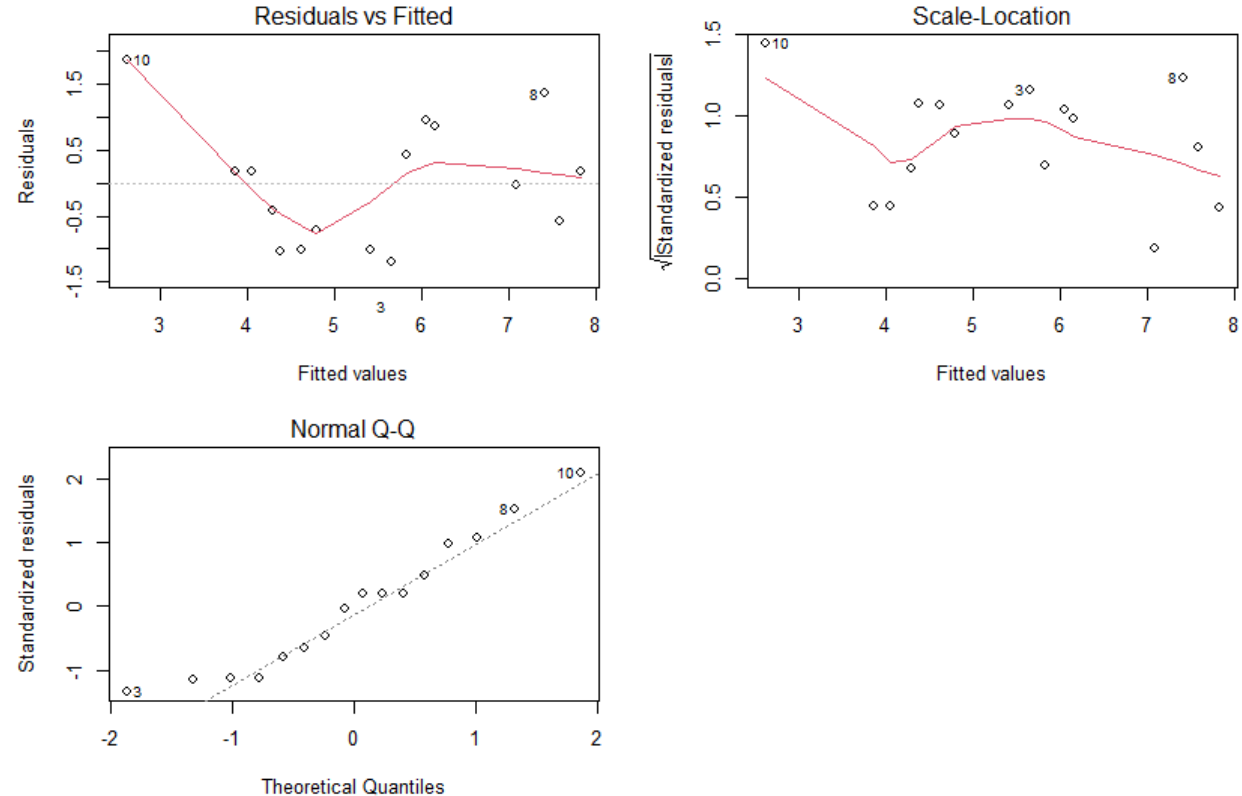

Figura 3.6. Gráficos de análisis de residuos modelo ANOVA Tc=80 minutos. 
Con el objetivo de verificar que las conclusiones obtenidas a partir del ANOVA paramétrico son correctas, se ha realizado un ANOVA robusto que permite obtener conclusiones cuando no se cumplen los supuestos del método paramétrico. Los resultados obtenidos con el método robusto se muestran en la Tabla 3.9. Comparando los resultados del ANOVA clásico y el robusto se observa que para un tiempo de corte de 5 minutos los resultados entre ambas pruebas coinciden excepto para el parámetro $a_{p}$ que es significativo, en el ANOVA robusto, para un nivel de significación de 0.1. Si se comparan los resultados para un tiempo de corte de 50 minutos Vc no es significativo y el resto de parámetros son significativos considerando un nivel de significación de 0.1. Por último, considerando el tiempo de corte de 80 minutos los resultados obtenidos con ambas pruebas coinciden.

Tabla 3.9. Resultados ANOVA robusto.

\begin{tabular}{|c|c|c|c|c|c|c|}
\hline \multirow[b]{2}{*}{ Factor } & \multicolumn{2}{|c|}{$\mathrm{Tc}=5 \mathrm{~min}$} & \multicolumn{2}{|c|}{$\mathrm{Tc}=50 \mathrm{~min}$} & \multicolumn{2}{|c|}{$\mathrm{Tc}=80 \mathrm{~min}$} \\
\hline & $\mathrm{F}$ & p-valor & $\mathrm{F}$ & p-valor & $\mathrm{F}$ & p-valor \\
\hline $\mathrm{Fz}$ & 8.06 & $0.017^{*}$ & 4.31 & 0.098 & 1.64 & 0.229 \\
\hline $\mathrm{Vc}$ & 6.87 & $0.025^{*}$ & 3.08 & 0.162 & 0.79 & 0.395 \\
\hline$a_{p}$ & 4.20 & 0.069 . & 4.83 & 0.052 . & 2.49 & 0.145 \\
\hline $\mathrm{Fv}$ & 8.26 & $0.016^{*}$ & 6.04 & $0.034^{*}$ & 4.45 & 0.061 . \\
\hline Fo & 28.77 & $3.17 \mathrm{E}-4^{* * * *}$ & 22.65 & $7.7 \mathrm{E}-4^{* * * *}$ & 14.60 & $3.36 \mathrm{E}-4^{*}$ \\
\hline
\end{tabular}

A partir de los resultados obtenidos en los análisis realizados se puede concluir que las mejores condiciones de rugosidad se obtienen con unas condiciones de mecanizado correspondientes a una velocidad de corte $(\mathrm{Vc}) \mathrm{de}$ $300 \mathrm{~m} / \mathrm{min}$, una profundidad de pasada $\left(a_{p}\right)$ de $0.5 \mathrm{~mm}$, un avance por diente (Fz) de $0.1 \mathrm{~mm} /$ diente, una orientación de la fibra $(\mathrm{Fo})$ de $90^{\circ}$ y un volumen de fibra ( $\mathrm{Fv}$ ) de $40 \%$ para un tiempo (Tc) de 5 minutos, con valores de rugosidad por debajo de las 4.5 micras. Para un tiempo de corte de 50 minutos coinciden las conclusiones excepto para la velocidad de corte que no resulta significativa. Finalmente, para un tiempo de corte de 80 minutos los únicos que factores que afectan a la rugosidad son la orientación y el volumen de fibra.

\subsubsection{Regresión cuantil}

La regresión cuantil tiene la ventaja, tal como se ha presentado en el Capítulo 2, de la no necesidad de cumplimiento de los supuestos de normalidad y homocedasticidad que requieren otros modelos de regresión clásicos permitiendo caracterizar la relación existente entre diferentes variables 
explicativas y una variable dependiente. En este apartado se presentan los resultados obtenidos de la caracterización de la relación entre la mediana (cuantil 0.5) de la rugosidad y las diferentes condiciones de mecanizado y del material. Para ello se han tomado las 15 medidas de cada experimento en cada tiempo de corte de forma independiente y no considerando la media de los 15 valores.

En la Figura 3.7 se muestra el histograma de la rugosidad y la función de densidad empírica donde se observa la presencia de asimetría positiva no verificándose el supuesto de normalidad requerido en el modelo lineal de regresión. Dicha asimetría se puede observar también en el gráfico BoxWhisker mostrado en la Figura 3.8 y en la Tabla 3.10, que muestra los estadísticos más relevantes.

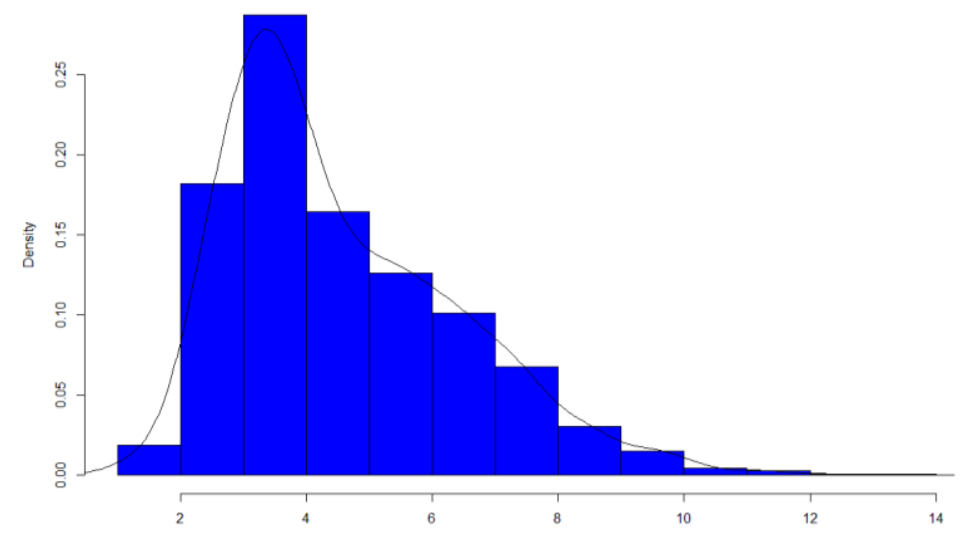

Figura 3.7. Histograma, función de densidad empírica de Ra.

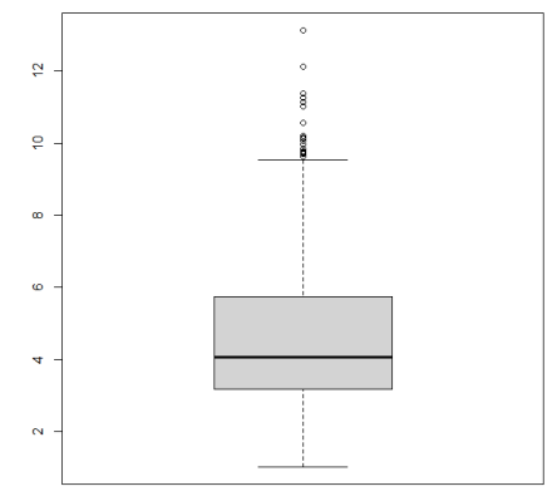

Figura 3.8. Diagrama Box-Whisker de Ra. 
Tabla 3.10. Resumen descriptivo de Ra.

\begin{tabular}{ccccc}
\hline Mínimo & $1^{\circ}$ Cuartil & Mediana & $3^{\circ}$ Cuartil & Máximo \\
\hline 1.020 & 3.190 & 4.070 & 5.746 & 13.120 \\
\hline
\end{tabular}

En la Tabla 3.11 se muestran los resultados obtenidos en los modelos de regresión. Como se observa en la tabla, la cual incluye los valores estimados de los coeficientes $\beta \mathrm{i}$, el estadístico t y el $\mathrm{p}$-valor, el modelo final obtenido incluye todas las variables explicativas, puesto que el p-valor es menor de 0.05.

Tabla 3.11. Resultados del modelo correspondiente al cuantil 0.50.

\begin{tabular}{cccc}
\hline Variable & Valor & t-valor & p-valor \\
\hline Intercept & 2.171 & 6.682 & $<0.0001$ \\
Fv & 0.039 & 10.735 & $<0.0001$ \\
Fo & -0.048 & -25.08 & $<0.0001$ \\
Vc & 0.003 & 7.941 & $<0.0001$ \\
Fz & 2.286 & 9.551 & $<0.0001$ \\
$\mathrm{a}_{\mathrm{p}}$ & 0.753 & 9.997 & $<0.0001$ \\
$\mathrm{Tc}$ & 0.019 & 13.357 & $<0.0001$ \\
\hline
\end{tabular}

La Figura 3.9 muestra la relación entre los diferentes cuantiles de la rugosidad y los parámetros incluidos en el análisis. Se puede observar que la pendiente de la recta de cada cuantil es distinta, lo que puede interpretarse como que los diferentes predictores influyen de forma distinta a cada cuantil de la rugosidad. En la misma figura se presenta el valor de los coeficientes de regresión estimados para los diferentes predictores mediante un modelo de regresión lineal ajustado por mínimos cuadrados ordinarios (línea roja horizontal). En ambos casos se representa el intervalo de confianza del 95\%. Así, la nube de puntos sombreada representa los valores inferiores y superiores del intervalo de confianza del estimador de la regresión cuantil y las bandas representadas en rojo corresponde al intervalo de confianza de la regresión por mínimos cuadrados ordinario.
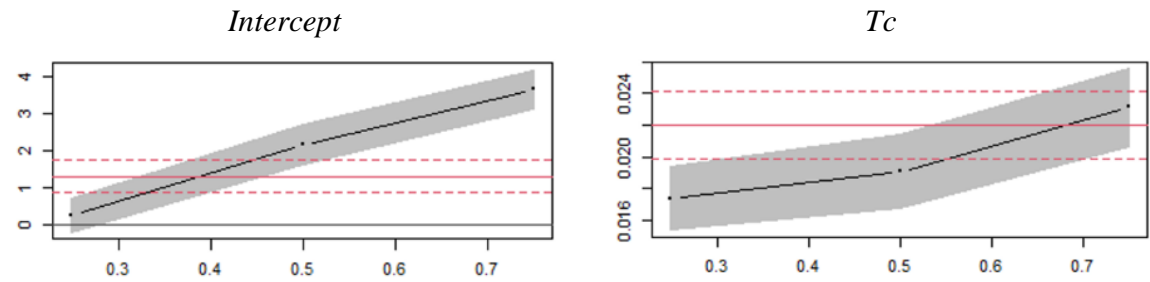
$F z$

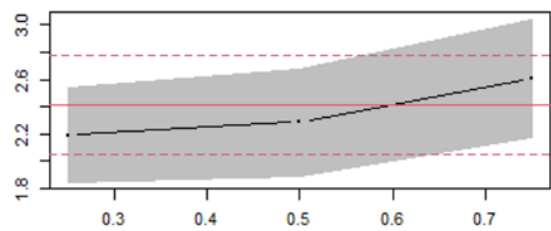

7

$F v$

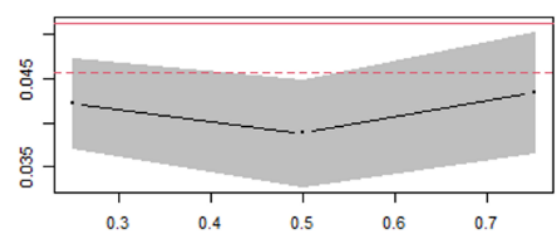

$V c$

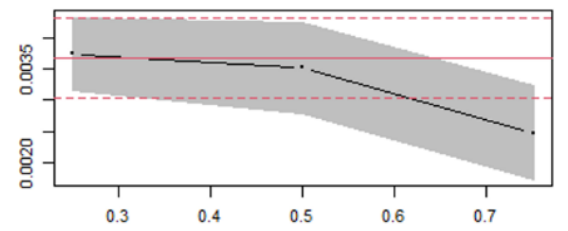

$a_{p}$

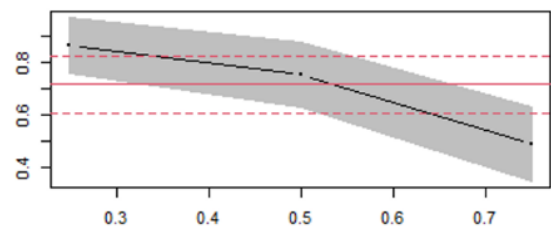

Fo

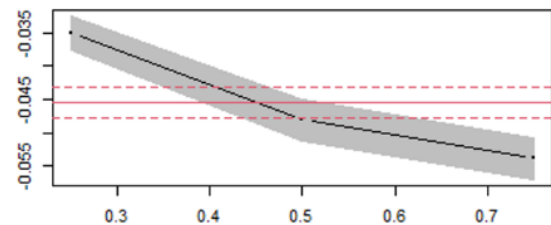

Figura 3.9. Cuantiles Ra vs parámetros de mecanizado y fibra.

En el modelo ajustado se observa que la rugosidad superficial (Ra) aumenta para las piezas con mayor volumen de fibra $(\mathrm{Fv})$ y disminuye para las piezas con una orientación de la fibra $(\mathrm{Fo})$ de $0^{\circ}-90^{\circ}$.

En relación a las condiciones de mecanizado, en los experimentos estudiados, al aumentar el avance $(\mathrm{Fz})$ y la profundidad de pasada $\left(\mathrm{a}_{\mathrm{p}}\right)$, se produce un aumento de la rugosidad.

En las Figuras 3.10, 3.11 y 3.12 se muestran los resultados obtenidos en la predicción para los diferentes tiempos de corte $(\mathrm{Tc}=5,50$ y $80 \mathrm{~min})$ y los intervalos de confianza $(1-\alpha=0.95)$ para cada una de las predicciones. Como el análisis se ha realizado con las mediciones individuales en lugar de las medias, en las figuras se observan intervalos amplios de variabilidad hasta aproximadamente 5 micras. 


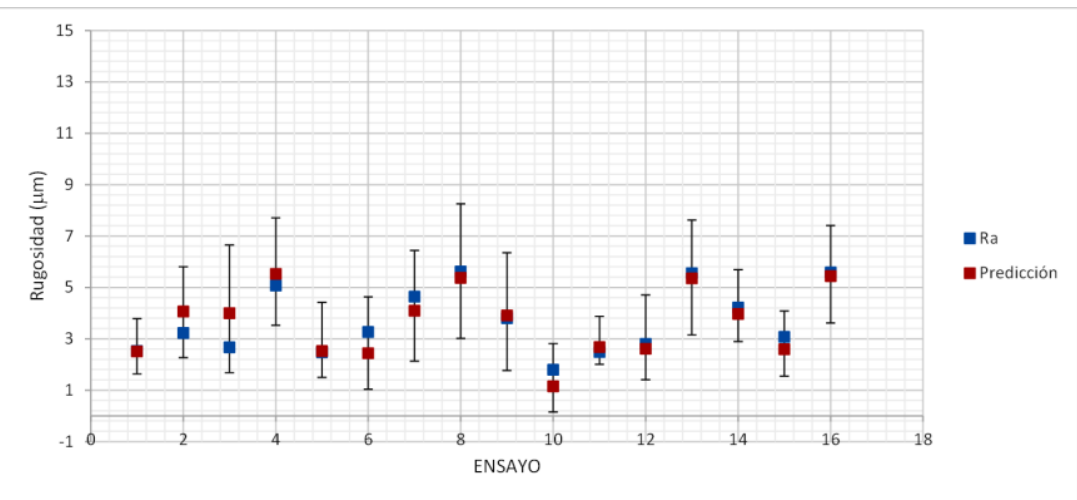

Figura 3.10. Rugosidad mediana observada, estimada e intervalo de confianza para cada uno de los ensayos $(T c=5 \mathrm{~min})$.

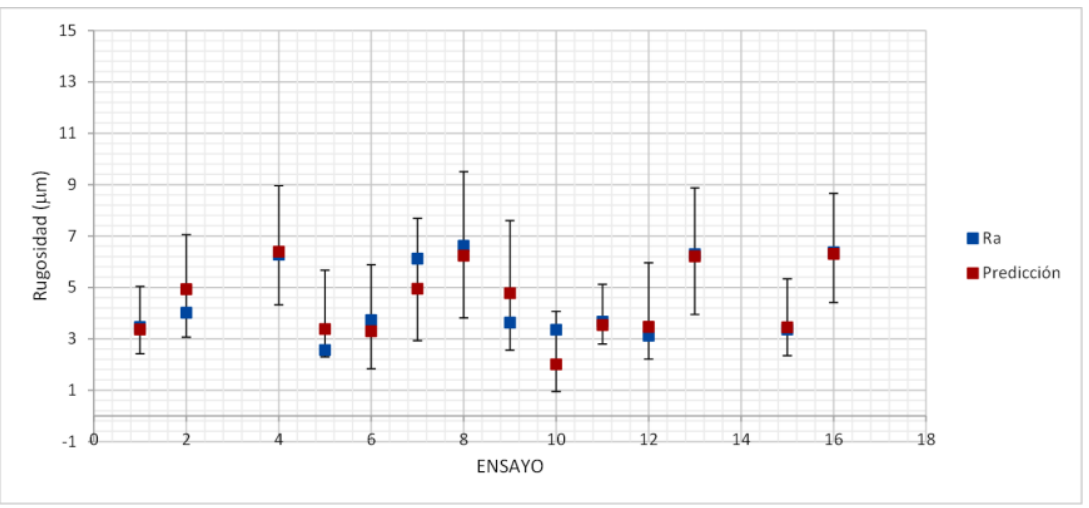

Figura 3.11. Rugosidad mediana observada, estimada e intervalo de confianza para cada uno de los ensayos $(T c=50 \mathrm{~min})$.

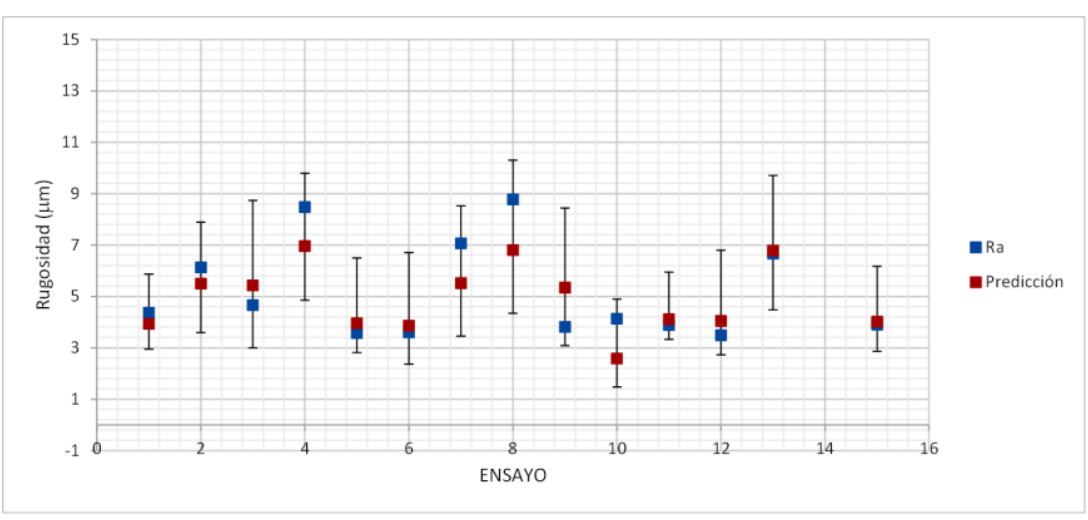

Figura 3.12. Rugosidad mediana observada, estimada e intervalo de confianza para cada uno de los ensayos $(T c=80 \mathrm{~min})$. 


\subsection{DESGASTE DE LA HERRAMIENTA}

Las fibras de los materiales compuestos pueden ser abrasivas y provocan un rápido desgaste de los filos de la herramienta de corte. La Tabla 3.12 muestra los datos obtenidos del desgaste del flanco $(\mathrm{Vb})$ para cada experimento y en cada tiempo de mecanizado. El desgaste se ha medido sobre los dos filos de corte y se ha seleccionado el valor mayor (Anexo II.3).

Tabla 3.12. Valor más desfavorable de desgaste por experimento.

\begin{tabular}{|c|c|c|c|c|c|c|c|c|}
\hline & \multirow{2}{*}{ Exp } & \multirow{2}{*}{ Condiciones } & \multicolumn{5}{|c|}{$\mathrm{Tc}$} & \multirow[b]{2}{*}{$80 \mathrm{~min}$} \\
\hline & & & $5 \min$ & $30 \mathrm{~min}$ & $50 \mathrm{~min}$ & $60 \mathrm{~min}$ & $70 \mathrm{~min}$ & \\
\hline & E01 & $\begin{array}{l}\text { Vc } 470 ; \mathrm{Fz} 0.1 ; \mathrm{a}_{\mathrm{p}} 1.5 \\
\text { Fo } 90^{\circ} ; \mathrm{Fv}=40 \%\end{array}$ & 0.061 & 0.065 & 0.069 & 0.075 & 0.078 & 0.082 \\
\hline & E02 & $\begin{array}{l}\text { Vc } 300 ; \mathrm{Fz} 0.1 ; \mathrm{a}_{\mathrm{p}} 1.5 \\
\text { Fo } 45^{\circ} ; \mathrm{Fv}=40 \%\end{array}$ & 0.024 & 0.043 & 0.046 & 0.056 & 0.061 & 0.066 \\
\hline & E03 & $\begin{array}{l}\text { Vc } 300 ; \mathrm{Fz} 0.4 ; \mathrm{a}_{\mathrm{p}} 0.5 \\
\text { Fo } 45^{\circ} ; \mathrm{Fv}=40 \%\end{array}$ & 0.026 & 0.030 & 0.034 & 0.045 & 0.052 & 0.055 \\
\hline & E04 & $\begin{array}{l}\text { Vc 300; Fz 0.4; } a_{p} 1.5 \\
\text { Fo } 45^{\circ} ; \quad F v=60 \%\end{array}$ & 0.054 & 0.072 & 0.075 & 0.078 & 0.084 & 0.085 \\
\hline & E05 & $\begin{array}{l}\text { Vc } 470 ; \mathrm{Fz} 0.1 ; \mathrm{a}_{\mathrm{p}} 0.5 \\
\text { Fo } 90^{\circ} ; \mathrm{Fv}=60 \%\end{array}$ & 0.049 & 0.052 & 0.057 & 0.063 & 0.067 & 0.072 \\
\hline & E06 & $\begin{array}{l}\text { Vc } 470 ; \mathrm{Fz} 0.4 ; \mathrm{a}_{\mathrm{p}} 0.5 \\
\text { Fo } 90^{\circ} ; \mathrm{Fv}=40 \%\end{array}$ & 0.083 & 0.087 & 0.091 & 0.102 & 0.113 & 0.117 \\
\hline $\mathrm{Vb}$ & E07 & $\begin{array}{l}\text { Vc } 300 ; \mathrm{Fz} 0.1 ; \mathrm{a}_{\mathrm{p}} 0.5 \\
\text { Fo } 45^{\circ} ; \mathrm{Fv}=60 \%\end{array}$ & 0.022 & 0.028 & 0.032 & 0.041 & 0.046 & 0.049 \\
\hline $\max$ & E08 & $\begin{array}{l}\text { Vc } 470 ; \mathrm{Fz} 0.4 ; \mathrm{a}_{\mathrm{p}} 0.5 \\
\text { Fo } 45^{\circ} ; \mathrm{Fv}=60 \%\end{array}$ & 0.078 & 0.081 & 0.084 & 0.095 & 0.097 & 0.101 \\
\hline \multirow[t]{8}{*}{$(\mathrm{mm})$} & E09 & $\begin{array}{l}\text { Vc } 470 ; \mathrm{Fz} 0.1 ; \mathrm{a}_{\mathrm{p}} 0.5 \\
\text { Fo } 45^{\circ} ; \mathrm{Fv}=40 \%\end{array}$ & 0.024 & 0.027 & 0.031 & 0.038 & 0.045 & 0.049 \\
\hline & E10 & $\begin{array}{l}\text { Vc } 300 ; \mathrm{Fz} 0.1 ; \mathrm{a}_{\mathrm{p}} 0.5 \\
\text { Fo } 90^{\circ} ; \mathrm{Fv}=40 \%\end{array}$ & 0.026 & 0.030 & 0.037 & 0.044 & 0.047 & 0.052 \\
\hline & E11 & $\begin{array}{c}\text { Vc } 300 ; \mathrm{Fz} 0.1 ; \mathrm{a}_{\mathrm{p}} 1.5 \\
\text { Fo } 90^{\circ} ; \mathrm{Fv}=60 \%\end{array}$ & 0.032 & 0.039 & 0.047 & 0.058 & 0.066 & 0.073 \\
\hline & E12 & $\begin{array}{l}\text { Vc } 300 ; \mathrm{Fz} 0.4 ; \mathrm{a}_{\mathrm{p}} 0.5 \\
\text { Fo } 90^{\circ} ; \mathrm{Fv}=60 \%\end{array}$ & 0.041 & 0.046 & 0.048 & 0.056 & 0.060 & 0.063 \\
\hline & E13 & $\begin{array}{l}\text { Vc } 470 ; \mathrm{Fz} 0.4 ; \mathrm{a}_{\mathrm{p}} 1.5 \\
\text { Fo } 45^{\circ} ; \mathrm{Fv}=40 \%\end{array}$ & 0.103 & 0.117 & 0.122 & 0.131 & 0.140 & 0.148 \\
\hline & E14 & $\begin{array}{l}\text { Vc } 470 ; \mathrm{Fz} 0.4 ; \mathrm{a}_{\mathrm{p}} 1.5 \\
\text { Fo } 90^{\circ} ; \mathrm{Fv}=60 \%\end{array}$ & 0.211 & 0.247 & - & - & - & - \\
\hline & E15 & $\begin{array}{c}\text { Vc } 300 ; \mathrm{Fz} 0.4 ; a_{\mathrm{p}} 1.5 \\
\text { Fo } 90^{\circ} ; \mathrm{Fv}=40 \%\end{array}$ & 0.045 & 0.045 & 0.047 & 0.052 & 0.056 & 0.058 \\
\hline & E16 & $\begin{array}{l}\text { Vc } 470 ; \mathrm{Fz} 0.1 ; \mathrm{a}_{\mathrm{p}} 1.5 \\
\text { Fo } 45^{\circ} ; \mathrm{Fv}=60 \%\end{array}$ & 0.052 & 0.052 & 0.059 & 0.067 & 0.071 & 0.073 \\
\hline
\end{tabular}


En la Figura 3.13 se muestra la representación gráfica de los valores obtenidos en los diferentes experimentos. Conforme el tiempo de corte aumenta, el desgaste también aumenta. Sin embargo, hasta el tiempo de corte de 80 minutos se observa que las gráficas son bastante planas y aun no se ha alcanzado el crecimiento exponencial típico del desgaste de flanco (Vb). Los valores máximos del desgaste $\mathrm{Vb}$ obtenidos para el tiempo de corte de 80 minutos no superan los $0.15 \mathrm{~mm}$, excepto para el experimento E14 que como ya se ha comentado, presenta una situación de desgaste excesiva en poco tiempo [103].

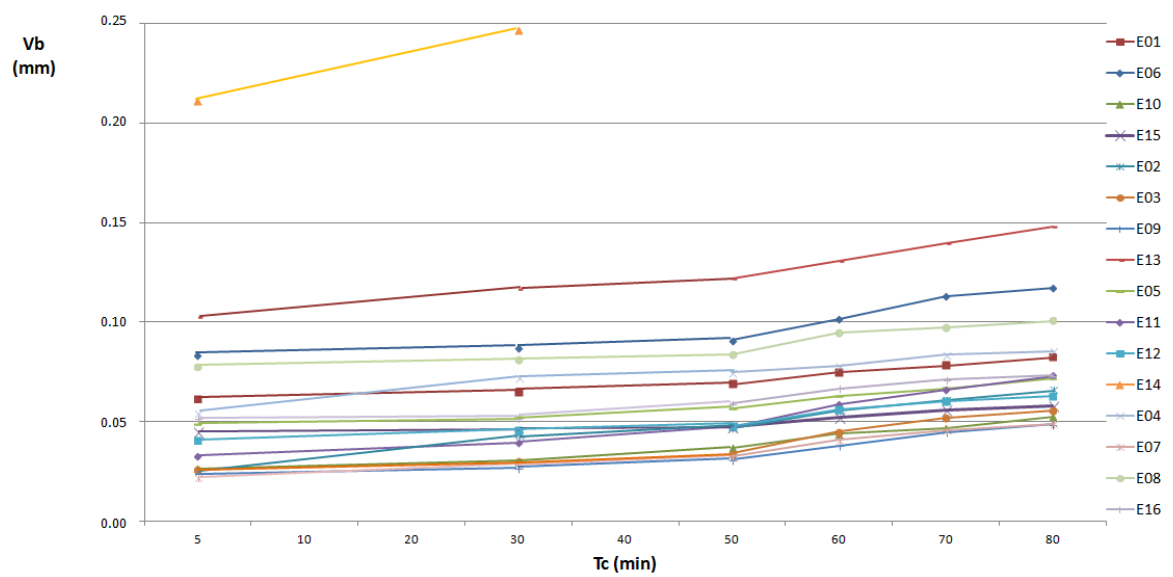

Figura 3.13. Desgaste de flanco $(\mathrm{Vb})$ frente a tiempo de corte $(T c)$.

\subsubsection{ANÁLISIS ESTADÍSTICO DEL DESGASTE}

De forma análoga al análisis de rugosidad realizado, se va a realizar un ANOVA de medidas repetidas con la finalidad de estudiar si el tiempo de corte es un factor significativo en el desgaste medio. Para comprobar este supuesto, como paso previo, se ha realizado la prueba de Mauchly, cuyo resultado se muestra en la Tabla 3.13 donde se pone de manifiesto el incumplimiento del supuesto de esfericidad ( $\mathrm{p}$-valor $<0.05$ ). Por esta razón se realizan las pruebas de Greenhouse-Geisser (GG) y Huynh-Feldt (HF) con corrección de esfericidad, cuyos resultados se muestran en la Tabla 3.14. Si se analizan los resultados de esta tabla se concluye, con las dos pruebas realizadas, que el tiempo de corte influye en el desgaste por lo que se ha optado, como en el caso de la rugosidad, por realizar el ANOVA para los tiempos de corte de 5, 50 y 80 minutos. La Figura 3.14 muestra el diagrama de Box-Whisker múltiple correspondiente al desgaste para los diferentes tiempos estudiados. 
Tabla 3.13. Test de esfericidad de Mauchly.

\begin{tabular}{lcc}
\hline Efecto & W & p-valor \\
\hline Tiempo de corte $(\mathrm{Tc})$ & 0.395 & $1.5 \mathrm{E}-3$ \\
\hline
\end{tabular}

Tabla 3.14. Resultados ANOVA de medidas repetidas con corrección de esfericidad.

\begin{tabular}{lcccc}
\hline Efecto & GGe & p-valor $(\mathrm{GG})$ & HFe & p-valor $(\mathrm{HF})$ \\
\hline Tiempo de corte $(\mathrm{Tc})$ & 0.677 & $8.97 \mathrm{E}-11$ & 0.724 & $2.22 \mathrm{E}-11$ \\
\hline
\end{tabular}

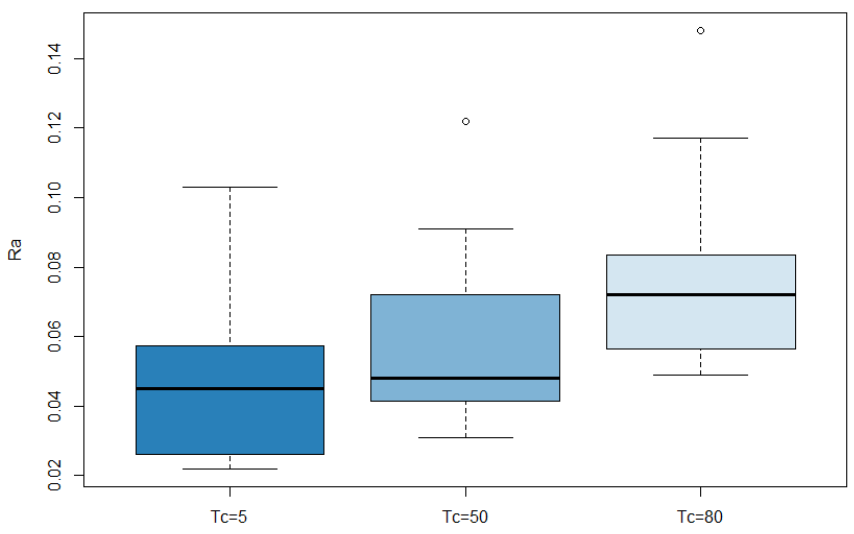

Figura 3.14. Desgaste observado (Vb) frente a tiempo de corte (Tc).

Una vez que se ha concluido la necesidad de realizar el estudio para los diferentes tiempos de corte se ha analizado el efecto de los de los parámetros de mecanizado (velocidad de corte, avance y profundidad de pasada) y de las características del material (fracción de volumen de fibra y orientación de la fibra) en el desgaste de la herramienta. De forma similar al estudio realizado para la rugosidad, se ha realizado un ANOVA para los tres instantes de tiempo $\mathrm{Tc}=5,50$ y 80 minutos. Los resultados del análisis se muestran en las Tablas $3.15,3.16$ y 3.17 .

Tabla 3.15. Tabla ANOVA para Vb, Tc=5 minutos.

\begin{tabular}{cccccccc}
\hline Fuente & $\begin{array}{c}\text { Suma de } \\
\text { Cuadrados }\end{array}$ & gdl & $\begin{array}{c}\text { Cuadrado } \\
\text { Medio }\end{array}$ & F-ratio & p-valor & $\eta^{2}$ & $\eta^{2}$ Parcial \\
\hline Fz & $7.65 \mathrm{E}-3$ & 1 & $7.65 \mathrm{E}-3$ & 7.873 & $0.018^{*}$ & 0.229 & 0.440 \\
$\mathrm{Vc}$ & $9.51 \mathrm{E}-3$ & 1 & $9.51 \mathrm{E}-3$ & 9.775 & $0.011^{*}$ & 0.285 & 0.494 \\
$\mathrm{a}_{\mathrm{p}}$ & $3.42 \mathrm{E}-3$ & 1 & $3.42 \mathrm{E}-3$ & 3.519 & 0.090. & 0.103 & 0.260 \\
\hline
\end{tabular}




\begin{tabular}{cccccccc}
\hline Fuente & $\begin{array}{c}\text { Suma de } \\
\text { Cuadrados }\end{array}$ & gdl & $\begin{array}{c}\text { Cuadrado } \\
\text { Medio }\end{array}$ & F-ratio & p-valor & $\eta^{2}$ & $\eta^{2}$ Parcial \\
\hline Fo & $1.68 \mathrm{E}-3$ & 1 & $1.68 \mathrm{E}-3$ & 1.728 & 0.218 & 0.050 & 0.147 \\
Fv & $1.33 \mathrm{E}-3$ & 1 & $1.33 \mathrm{E}-3$ & 1.369 & 0.269 & 0.040 & 0.120 \\
Residuos & $9.72 \mathrm{E}-3$ & 9 & $5.50 \mathrm{E}-5$ & & & & \\
\hline
\end{tabular}

Tabla 3.16. Tabla ANOVA para Vb, Tc=50 minutos.

\begin{tabular}{cccccccc}
\hline Fuente & $\begin{array}{c}\text { Suma de } \\
\text { Cuadrados }\end{array}$ & gdl & $\begin{array}{c}\text { Cuadrado } \\
\text { Medio }\end{array}$ & F-ratio & p-valor & $\eta^{2}$ & $\eta^{2}$ Parcial \\
\hline Fz & $2.92 \mathrm{E}-3$ & 1 & $2.92 \mathrm{E}-3$ & 11.063 & $8.85 \mathrm{E}-03^{* *}$ & 0.318 & 0.551 \\
Vc & $3.57 \mathrm{E}-3$ & 1 & $3.57 \mathrm{E}-3$ & 13.528 & $5.09 \mathrm{E}-03^{* *}$ & 0.389 & 0.600 \\
$\mathrm{ap}$ & $1.36 \mathrm{E}-3$ & 1 & $1.36 \mathrm{E}-3$ & 5.154 & $0.049^{*}$ & 0.148 & 0.364 \\
Fo & $1.60 \mathrm{E}-5$ & 1 & $1.60 \mathrm{E}-5$ & 0.061 & 0.811 & 0.002 & 0.007 \\
Fv & $4.71 \mathrm{E}-5$ & 1 & $4.71 \mathrm{E}-5$ & 0.178 & 0.683 & 0.005 & 0.019 \\
Residuos & $2.37 \mathrm{E}-3$ & 9 & $1.20 \mathrm{E}-4$ & & & & \\
\hline
\end{tabular}

Tabla 3.17. Tabla ANOVA para Vb, Tc=80 minutos.

\begin{tabular}{cccccccc}
\hline Fuente & $\begin{array}{c}\text { Suma de } \\
\text { Cuadrados }\end{array}$ & gdl & $\begin{array}{c}\text { Cuadrado } \\
\text { Medio }\end{array}$ & F-ratio & p-valor & $\eta^{2}$ & $\eta^{2}$ Parcial \\
\hline Fz & $3.03 \mathrm{E}-3$ & 1 & $3.03 \mathrm{E}-3$ & 7.697 & $0.022^{*}$ & 0.284 & 0.461 \\
Vc & $3.87 \mathrm{E}-3$ & 1 & $3.87 \mathrm{E}-3$ & 9.824 & $0.012^{*}$ & 0.362 & 0.522 \\
$\mathrm{a}_{\mathrm{p}}$ & $1.23 \mathrm{E}-3$ & 1 & $1.23 \mathrm{E}-3$ & 3.120 & 0.111 & 0.115 & 0.257 \\
Fo & $7.10 \mathrm{E}-6$ & 1 & $7.10 \mathrm{E}-6$ & 0.018 & 0.896 & $7.0 \mathrm{E}-4$ & 0.002 \\
Fv & $5.80 \mathrm{E}-6$ & 1 & $5.80 \mathrm{E}-6$ & 0.012 & 0.914 & $4.0 \mathrm{E}-4$ & 0.001 \\
Residuos & $3.55 \mathrm{E}-3$ & 9 & & & & & \\
\hline
\end{tabular}

A partir de los resultados del ANOVA se concluye que, para los tiempos de corte estudiados, la velocidad de corte $(\mathrm{Vc})$ y el avance por diente $(\mathrm{Fz})$ tienen un efecto significativo sobre el desgaste del flanco $(\mathrm{Vb})$ siendo la velocidad de corte el factor más significativo.

Las conclusiones anteriores son válidas si se verifican los supuestos de normalidad y homocedasticidad cuya comprobación se ha realizado, al igual que en el análisis de rugosidad, mediante las pruebas de Saphiro Wilks y de Barlett. Los resultados de las dos pruebas se muestran en las Tablas $3.18 \mathrm{y}$ 3.19 . 
Tabla 3.18. Resultados test Saphiro-Wilks.

\begin{tabular}{ccc}
\hline Tc $(\min )$ & Estadístico & p-valor \\
\hline 5 & 0.714 & $2.43 \mathrm{E}-04$ \\
50 & 0.739 & $4.67 \mathrm{E}-04$ \\
80 & 0.765 & $9.79 \mathrm{E}-04$ \\
\hline
\end{tabular}

Tabla 3.19. Resultados prueba Barlett.

\begin{tabular}{ccccccc}
\hline & \multicolumn{2}{c}{$\mathrm{Tc}=5 \mathrm{~min}$} & \multicolumn{2}{c}{$\mathrm{Tc}=50 \mathrm{~min}$} & \multicolumn{2}{c}{$\mathrm{Tc}=80 \mathrm{~min}$} \\
\hline Factor & $\mathrm{K}^{2}$ & $\mathrm{p}$-valor & $\mathrm{K}^{2}$ & $\mathrm{p}$-valor & $\mathrm{K}^{2}$ & $\mathrm{p}$-valor \\
\hline $\mathrm{Fz}$ & 9.397 & $2.17 \mathrm{E}-4^{*}$ & 10.368 & $1.28 \mathrm{e}-3^{*}$ & 10.51 & $1.28 \mathrm{E}-3^{*}$ \\
$\mathrm{Vc}$ & 12.41 & $4.27 \mathrm{E}-4^{*}$ & 10.298 & $1.33 \mathrm{E}-3^{*}$ & 10.992 & $9.14 \mathrm{E}-4^{*}$ \\
$\mathrm{a}_{\mathrm{p}}$ & 4.70 & $0.030^{*}$ & 4.480 & $0.034^{*}$ & 3.202 & 0.073 \\
$\mathrm{Fv}$ & 2.845 & 0.091 & 2.010 & 0.156 & 0.833 & 0.361 \\
$\mathrm{Fo}$ & 2.983 & 0.084 & 2.135 & 0.144 & 1.337 & 0.247 \\
\hline
\end{tabular}

* p-valor $<0.05$

El análisis de los resultados de las pruebas de Saphiro Wilks y de Barlett muestra un incumplimiento de los supuestos de normalidad y homocedasticidad lo que conduce a la realización de un ANOVA robusto con el objetivo de verificar si las conclusiones obtenidas con el método paramétrico son válidas (Tabla 3.20). La comparación de los resultados obtenidos por ambos métodos, coinciden para todos los tiempos de corte analizados, por lo que las conclusiones obtenidas se dan por válidas.

Tabla 3.20. Resultados ANOVA robusto.

\begin{tabular}{ccccccc}
\hline & \multicolumn{2}{c}{$\mathrm{Tc}=5 \mathrm{~min}$} & \multicolumn{2}{c}{$\mathrm{Tc}=50 \mathrm{~min}$} & \multicolumn{2}{c}{$\mathrm{Tc}=80 \mathrm{~min}$} \\
\hline Factor & $\mathrm{F}$ & $\mathrm{p}$-valor & $\mathrm{F}$ & $\mathrm{p}$-valor & $\mathrm{F}$ & $\mathrm{p}$-valor \\
\hline $\mathrm{Fz}$ & 7.708 & $0.019^{*}$ & 9.006 & $0.014^{*}$ & 6.623 & $0.030^{*}$ \\
$\mathrm{Vc}$ & 17.977 & $1.71 \mathrm{E}-3^{* *}$ & 10.946 & $9.1 \mathrm{E}-3^{* *}$ & 8.278 & $0.018^{*}$ \\
$\mathrm{a}_{\mathrm{p}}$ & 25.153 & $5.25 \mathrm{E}-4^{* * *}$ & 4.173 & $0.071^{*}$ & 2.665 & 0.137 \\
$\mathrm{Fv}$ & 0.771 & 0.401 & 0.154 & 0.704 & 0.013 & 0.911 \\
Fo & 4.468 & 0.061. & 0.063 & 0.807 & 0.030 & 0.866 \\
\hline
\end{tabular}

Códigos significación p-valor: 0 ‘***’ 0.001 ‘**’ 0.01 ‘*’ 0.05 ‘’ 0.1 “ ’ 1 


\subsection{MODELO PREDICTIVO DE Ra}

Debido a la heterogeneidad del material, las medidas de la rugosidad superficial presentan variabilidad. Por ello, se va a intentar predecir su comportamiento mediante un análisis de regresión incluyendo como variables predictivas (variables independientes) las condiciones de mecanizado (Vc, Fz y $\mathrm{a}_{\mathrm{p}}$ ), el volumen y la orientación de la fibra (Fv, Fo), el tiempo de corte (Tc) y el desgaste $(\mathrm{Vb})$.

El ajuste del modelo se ha obtenido realizando el ajuste del modelo de regresión mediante un procedimiento stepwise. La elección del modelo se ha realizado con selección de variables backward, el ajuste se inicia con un modelo con todas las variables y se eliminan progresivamente hasta encontrar un modelo con todas las variables significativas, y forward, el modelo inicial no contiene ninguna variable predictiva y se van añadiendo variables hasta que no se omite ninguna variable que aporte información. La selección del modelo final se ha realizado en base al Criterio de Información de Akaike (AIC). Ambas regresiones, backward y forward, seleccionan el mismo modelo que incluye, tal como se muestra en la Tabla 3.21 cinco variables predictoras. El AIC del modelo seleccionado es igual a 106.79 y el valor de $\mathrm{R}^{2}$ ajustado de 0.80 .

Tabla 3.21. Resultados del modelo de regresión de la rugosidad en función del desgaste, el Tc y los parámetros de mecanizado y material.

\begin{tabular}{ccccc}
\hline Variable & Estimación & $\begin{array}{c}\text { Desviación } \\
\text { típica }\end{array}$ & $\mathrm{t}$ & $\mathrm{p}$-valor \\
\hline Intercept & 0.6616 & 0.57690 & 1.15 & 0.258 \\
Vb & 17.2753 & 3.09028 & 5.59 & $<0.001$ \\
Tc (min) & 0.0168 & 0.00348 & 4.81 & $<0.001$ \\
Fv & 0.0392 & 0.01063 & 3.69 & $<0.001$ \\
Fo (0-1) & -2.1582 & 0.21263 & -10.15 & $<0.001$ \\
\hline
\end{tabular}

Como paso previo a la interpretación del modelo, se procede a la realización del diagnóstico del mismo con el objetivo de analizar si el modelo ajustado representa de forma adecuada los datos. El diagnóstico se ha realizado a partir del análisis de los residuos los cuales deben tener una distribución normal de media cero, la misma varianza (homocedasticidad) y ser independientes. En la Tabla 3.22 se muestran los resultados de las pruebas de Saphiro-Wilks y F-test donde se observa que no se pueden rechazar los supuestos de normalidad y homocedasticidad ( $\mathrm{p}$-valor $>0-05$ ). La independencia de los residuos se 
comprueba mediante un test de autocorrelación obteniéndose un valor igual a 0.98 por lo que se puede asumir el cumplimiento del supuesto.

La verificación de los supuestos puede también realizarse de forma gráfica mostrándose los gráficos de residuos en la Figura 3.15. Destacar la presencia de un punto anómalo que corresponde al ensayo 14 con el que se obtiene un valor de rugosidad anormalmente elevado para las condiciones de dicho ensayo.

Tabla 3.22. Resultados test Saphiro-Wilks y F-test. Comprobación de supuestos modelo lineal.

\begin{tabular}{ccc}
\hline Prueba & Estadístico & p-valor \\
\hline Saphiro-Wilks & 0.967 & 0.2098 \\
F-test & 1.598 & 0.224 \\
\hline
\end{tabular}
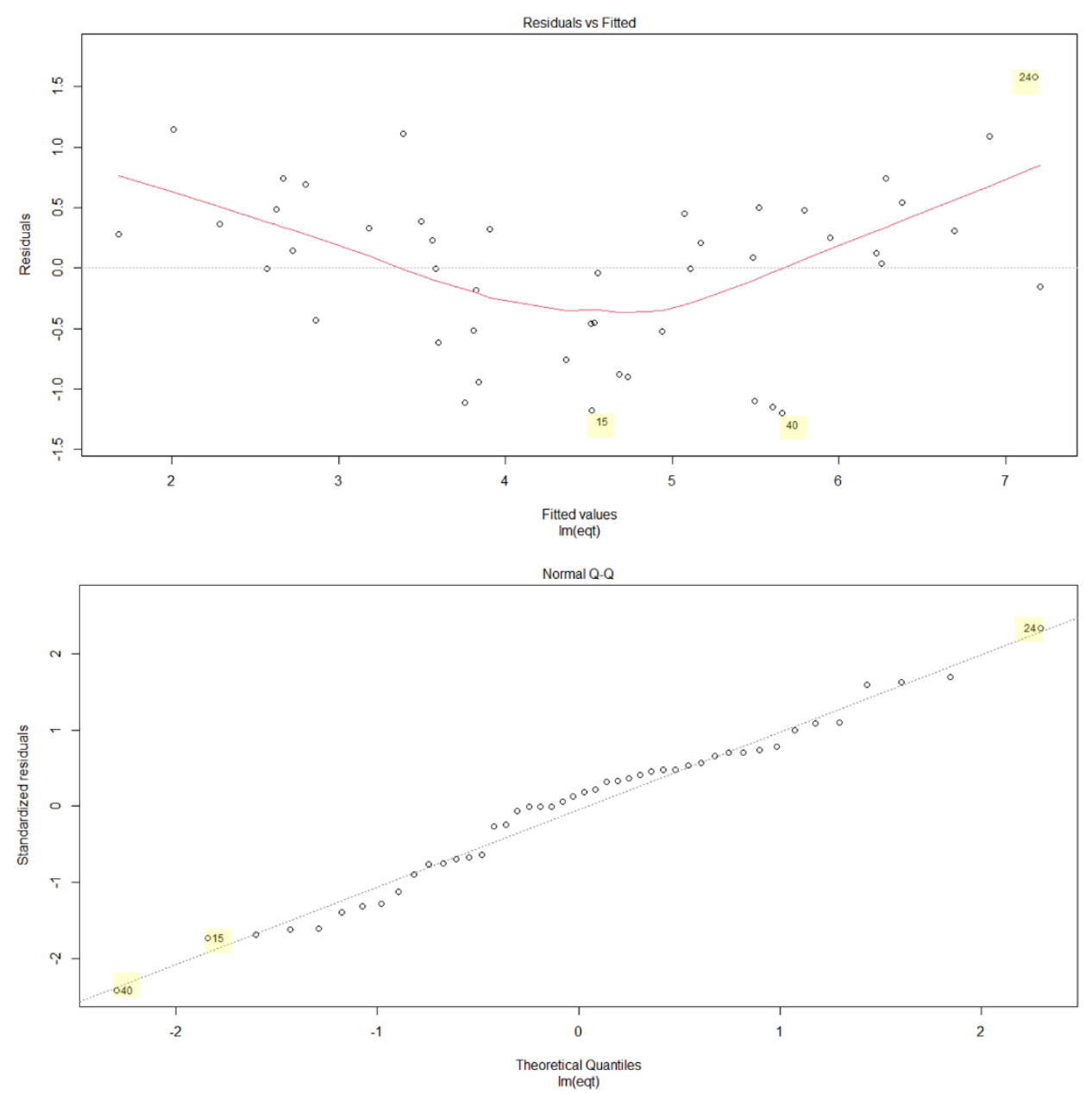

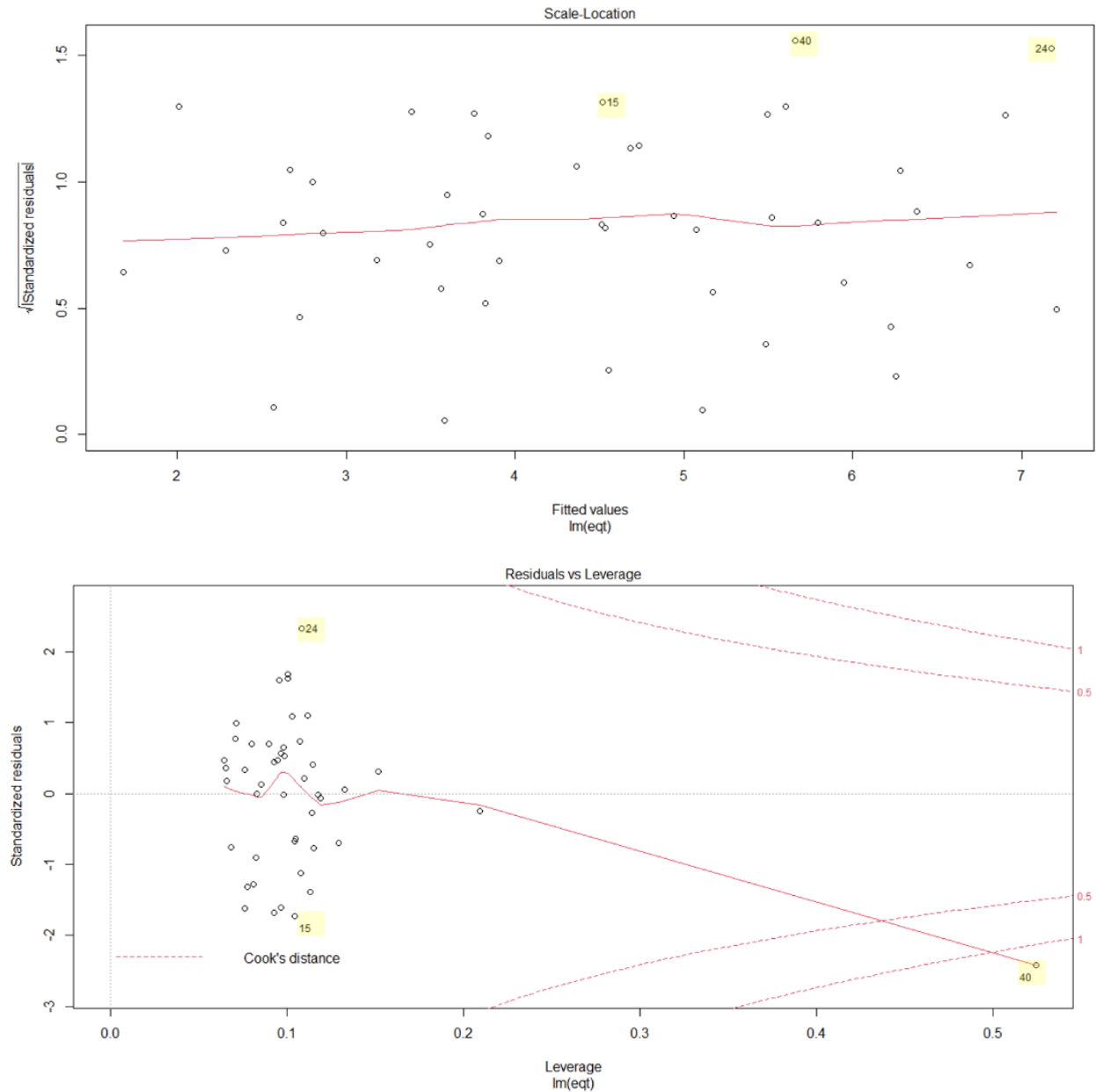

Figura 3.15. Gráficos de los residuos. Modelo de regresión lineal para la predicción de la Rugosidad media.

A nivel interpretativo, analizando el modelo ajustado, se observa que la rugosidad superficial está correlacionada positivamente con las variables $\mathrm{Vb}$, Fv y Tc y negativamente con la orientación de la fibra (Fo). Finalmente, en la Figura 3.16 se muestra los valores de la Ra observada y estimada con el modelo de regresión ajustado en función de $\mathrm{Vb}$. 


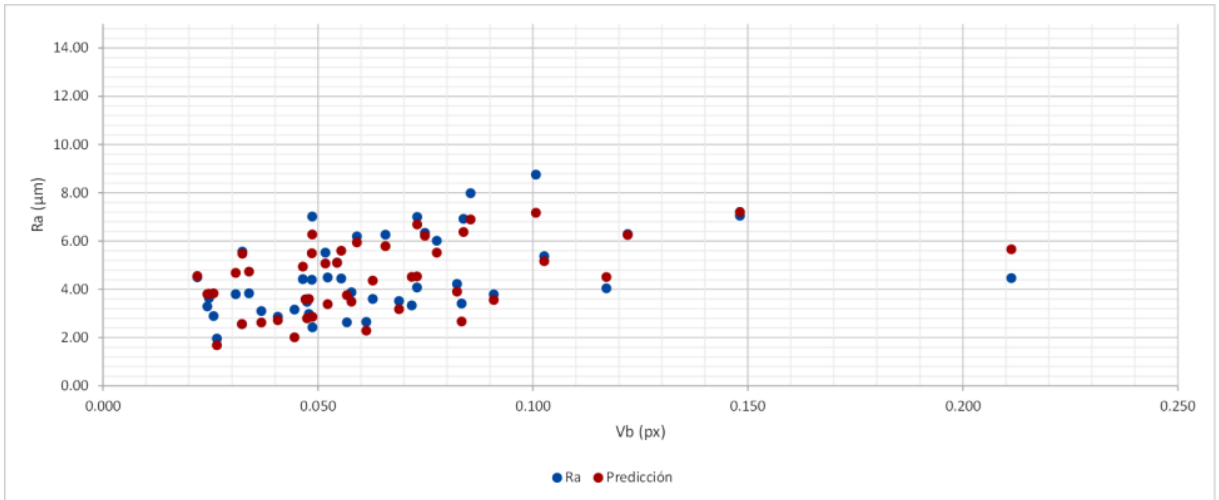

Figura 3.16. Rugosidad ( $R$ a) observada y predicha con el modelo de regresión frente al desgaste $(\mathrm{Vb})$. 


\section{CAPÍTULO 4. ANÁLISIS DEL DELAMINADO}

\subsection{INTRODUCCIÓN}

El delaminado es un problema que suele aparecer en el mecanizado de piezas de material compuesto reforzado con fibras, y que afecta dimensionalmente a la precisión y al posterior proceso de ensamblaje de la pieza fabricada. El delaminado consiste en la separación de las capas del material compuesto debido a la fuerza que ejerce la herramienta durante el proceso de mecanizado. En ocasiones, la presión de la herramienta produce una flexión de las fibras superficiales del tejido, en vez de cortarlas, lo que provoca un aspecto no uniforme del borde mecanizado de la pieza. En el contorneado de una pieza, la delaminación aparece en la capa superior e inferior del laminado, en las zonas donde no hay suficiente resina para sujetar el tejido.

En el proceso de fresado, el delaminado tiene dos efectos principales:

- hacia el interior de la pieza, con un daño producido sobre su superficie, ya que, al doblarse las mechas de fibra de las capas exteriores del material, se produce un desconchado o pérdida de la resina superficial.

- hacia el exterior del borde mecanizado de la pieza, con un aspecto deshilachado. Estas fibras crean un efecto de hilos sueltos y desordenados en exceso sobre la cota dimensional establecida para la pieza, obligando a 
retrabajar la pieza desbarbando el borde con una herramienta abrasiva, e impidiendo la verificación dimensional y un correcto ensamblaje del producto.

El delaminado puede clasificarse en tres tipos: I, II y III. El tipo I es el delaminado que se produce hacia el interior de la pieza y los tipos II y III los que se producen hacia el exterior de la pieza. En algunas zonas del borde mecanizado se dan simultáneamente ambos efectos, daños hacia el interior y hacia el exterior de la pieza. Esta combinación se conoce como delaminado tipo I/II.

Este capítulo se estructura en cuatro grandes bloques. En primer lugar, se exponen las consideraciones teóricas necesarias para comprender el delaminado y permitir el posterior análisis de los datos obtenidos experimentalmente. En segundo lugar, se realizará un estudio preliminar del delaminado. En tercer lugar, se llevará a cabo un estudio cuantitativo. En cuarto lugar, se hará un estudio estadístico del delaminado.

\subsection{CONSIDERACIONES TEÓRICAS}

Antes de mostrar los resultados obtenidos en los experimentos, se van a comentar algunos aspectos teóricos relacionados con el mecanizado de materiales compuestos reforzados con fibra y la aparición del delaminado. Es necesario conocer estos aspectos, porque posteriormente se utilizan para sustentar las conclusiones obtenidas en el estudio de delaminado. Estos aspectos son:

- Ángulos que caracterizan el proceso de fresado de los materiales compuestos reforzados con fibra, que se utilizarán como referencia relativa de posicionamiento.

- Orientación de la trayectoria de corte con respecto a las direcciones principales del tejido.

- La distancia del punto de incisión del filo de corte sobre la mecha de tejido, hasta el espacio o cruce entre dos mechas, que se define como parámetro $\mathrm{Xd}$.

\subsubsection{REFERENCIAS DE POSICIONAMIENTO ANGULAR}

La orientación de la fibra con respecto a la trayectoria de corte de la herramienta influye directamente en la apariencia del delaminado. Para estudiar este efecto, se van a definir diferentes ángulos, que permiten establecer la posición angular relativa de las hebras que forman el tejido de refuerzo con 
los movimientos fundamentales del proceso de mecanizado. De esta forma, se fija la orientación de la fibra respecto a la trayectoria de corte de la herramienta.

En la literatura también se han definido diferentes ángulos para caracterizar este proceso de fresado, aunque los distintos autores [67, 75, 79, 92 y 104], no han desarrollado las mismas relaciones que se muestran a continuación. No existe consenso sobre la nomenclatura utilizada, su definición y la relación que existe entre los ángulos a la hora de explicar el efecto de la orientación de la fibra en el delaminado. Por otro lado, cuando se han definido estos ángulos para la operación de ranurado, no se han mantenido las referencias de la orientación de la fibra. Por ello, en este punto de la tesis ha sido necesario definir ángulos que se puedan utilizar para las diferentes operaciones de fresado como el contorneado y el ranurado. En la tesis se plantea una definición completa y robusta de estos ángulos y sus relaciones, aplicable a ambas operaciones. Los ángulos necesarios para caracterizar el proceso de fresado de materiales compuestos reforzados con fibra son los siguientes [105]:

- Ángulo de empañe o engagement angle $(\varphi)$.

- Ángulo de orientación de la fibra o fiber orientación angle $(\phi)$.

- Ángulo de corte o fiber cutting angle $(\chi)$.

En este primer momento, estos ángulos se van a particularizar para el fresado de los laminados ensayados en la tesis, es decir, para una operación de fresado periférico en concordancia, donde el movimiento de corte (Mc) lo lleva la herramienta y el movimiento de avance (Ma) lo lleva la pieza. Esto condiciona directamente los valores angulares que marcan el desarrollo de los experimentos y que se van a identificar a continuación.

El ángulo de empañe $(\varphi)$ es el ángulo que forman entre sí los movimientos fundamentales (Figura 4.1). Se define desde el movimiento de avance hasta el movimiento de corte, en sentido antihorario. Al iniciarse el corte, cuando el diente entra a cortar sobre la pieza, el ángulo de empañe coincide con el ángulo de fresado $(\Theta)$ y su valor se va reduciendo con el giro de la fresa hasta que coinciden las direcciones de los movimientos fundamentales. La velocidad de corte cambia de dirección en cada instante con lo que el ángulo de empañe varía continuamente con el giro de la fresa.

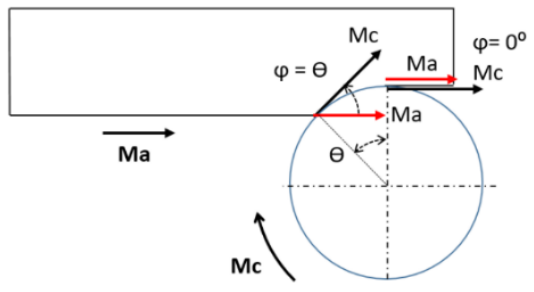

Figura 4.1. Ángulo de empañe $\varphi$ 
El ángulo de orientación de la fibra $(\phi)$ permite conocer la orientación de las hebras del tejido con respecto al movimiento de avance. Se define desde la dirección de orientación de la fibra hasta el movimiento de avance, en sentido antihorario. Para definir el ángulo de orientación de la fibra $\phi$ se ha elegido como sentido positivo la dirección hacia fuera del borde mecanizado en concordancia. En los experimentos realizados en la tesis, tiene valores fijos $\left(45^{\circ}, 90^{\circ}\right.$ y $\left.135^{\circ}\right)$, aunque podría variar si la trayectoria de corte de la herramienta variase con respecto a la disposición del tejido (Figura 4.2).

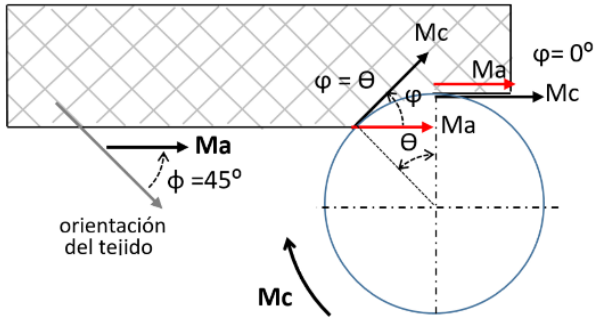

(a) $\varphi \operatorname{para} \phi=45^{\circ}$

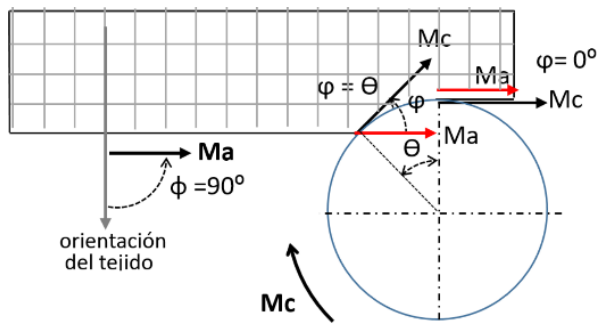

(b) $\varphi$ para $\phi=90^{\circ}$

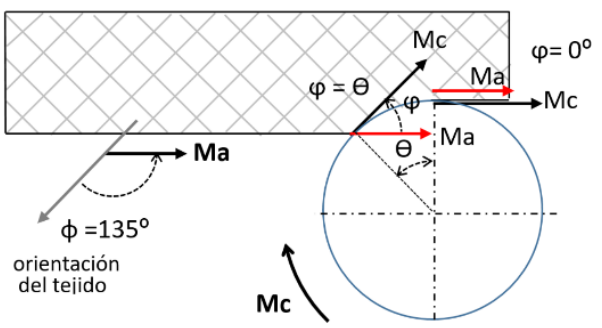

(c) $\varphi$ para $\phi=135^{\circ}$

Ángulo de orientación de fibra $\phi$ con ángulo de empañe $\varphi$

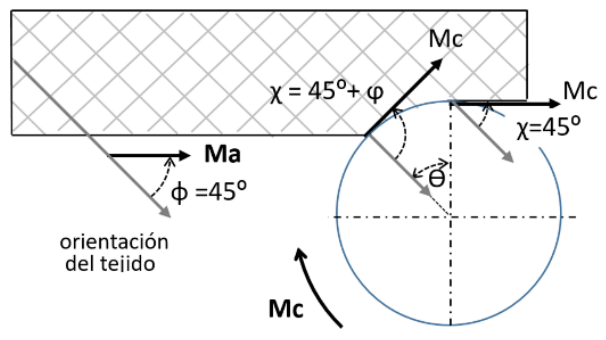

(d) $\chi$ para $\phi=45^{\circ}$

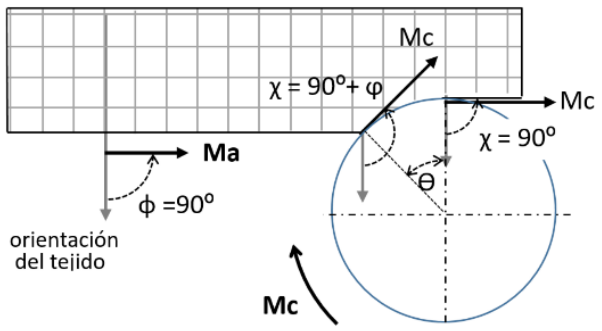

(e) $\chi$ para $\phi=90^{\circ}$

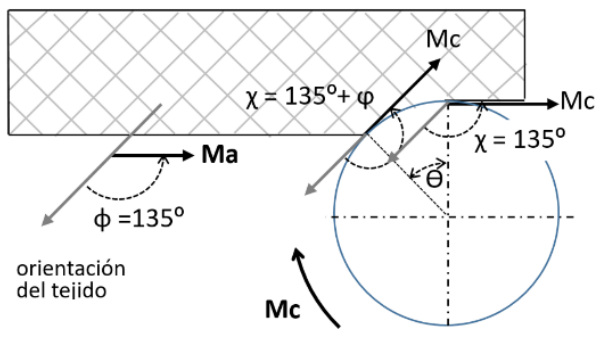

(f) $\chi$ para $\phi=135^{\circ}$

Angulo de corte $\chi$ con la dirección principal de tejido

Figura 4.2. Variación de los ángulos de referencia $\phi, \varphi y \chi$. 
El ángulo de corte $(\chi)$ se define como el ángulo formado desde la orientación de la fibra hasta el movimiento de corte, en sentido antihorario. Este ángulo permite conocer cómo incide la herramienta sobre la fibra y, en consecuencia, la dirección de aplicación de la fuerza principal de corte. Dado que la velocidad de corte cambia de dirección con el giro de la fresa en cada instante, el ángulo de corte también varía continuamente con el giro de la fresa.

Los ángulos que se han definido anteriormente están relacionados entre sí (ecuación 4.1). En la Figura 4.3 se resumen las distintas configuraciones para la operación de contorneado en concordancia si el movimiento de avance (Ma) lo lleva la pieza. El ángulo de empañe $(\varphi)$ al inicio del corte siempre coincide con el ángulo de fresado $(\Theta)$ y se va reduciendo hasta que al final del corte es cero. El ángulo de corte $(\chi)$, que representa la orientación de la fibra con el movimiento de corte $(\mathrm{Mc})$, siempre es el resultado de la suma del ángulo de orientación de la fibra $(\phi)$ más el ángulo de empañe $(\varphi)$.

$$
\chi=\phi+\varphi
$$

(ecuación 4.1)

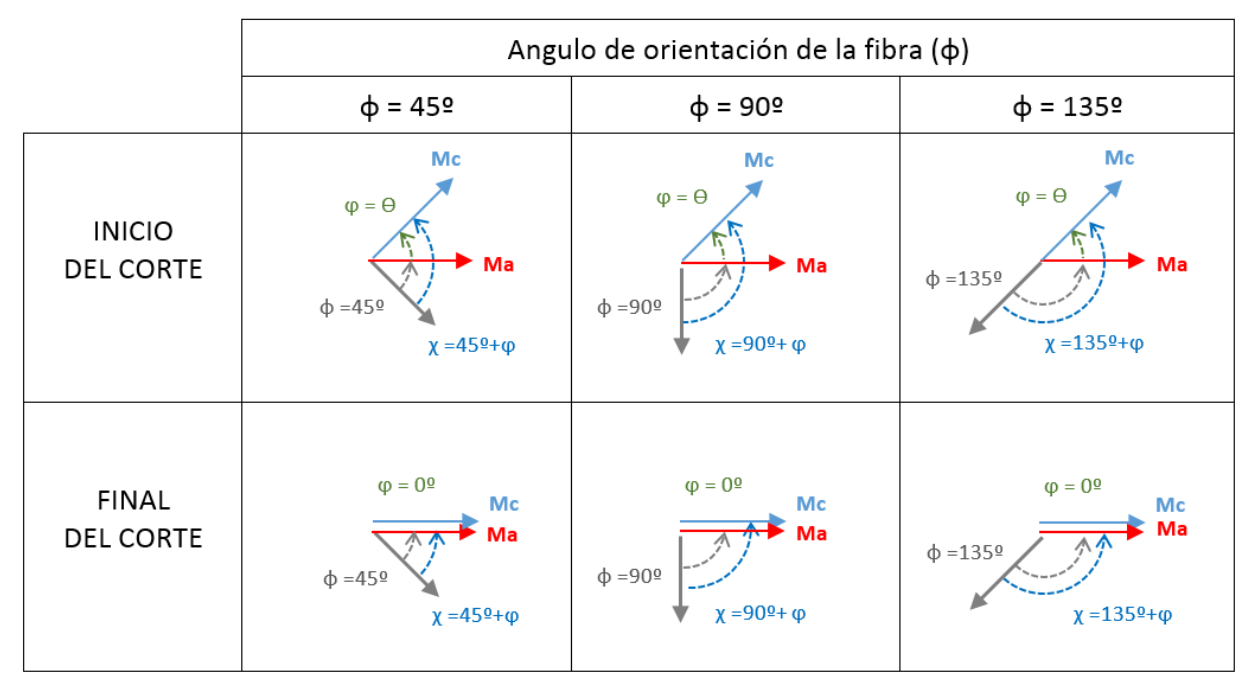

Figura 4.3. Ángulos de referencia al inicio y al final del corte.

Observando la geometría de los experimentos, se puede concluir que al final del corte de la fresa, el ángulo de corte $(\chi)$ coincide con el ángulo de orientación de la fibra $(\phi)$, porque en ese punto de mecanizado el ángulo de empañe $(\varphi)$ siempre es igual a cero.

Estas relaciones se han deducido considerando que el movimiento de corte (Mc) lo lleva la herramienta y el movimiento de avance (Ma) lo lleva la pieza. En el caso en que el movimiento de avance lo lleve también la herramienta, en 
lugar de la pieza, la dirección del movimiento de avance difiere en $180^{\circ}$ con lo expuesto anteriormente, manteniéndose el resto de relaciones entre los distintos ángulos, incluido el ángulo de corte $(\chi)$.

\subsubsection{Aplicación al ranurado}

La definición de los ángulos propuesta para el contorneado se puede aplicar también a la operación de ranurado, donde se presentan los dos modos de fresado: en concordancia y en oposición. Aunque diferentes autores incluyen referencias parciales a estos ángulos [67, 75, 79, 92 y 104], en ellas no se define un origen común de referencia para la orientación de las fibras, situando la dirección principal del tejido al revés en concordancia qué en oposición. Por ello las relaciones que proponen entre estos ángulos están condicionadas al tipo de fresado, y no se cumplen para determinadas posiciones angulares.

Con la propuesta realizada en esta tesis (Figura 4.4), se puede identificar la variación del ángulo de empañe $(\varphi)$, el ángulo de orientación de la fibra $(\phi)$ y el ángulo de corte $(\chi)$ en el mecanizado de una ranura, manteniéndose siempre la relación anterior (ecuación 4.1), con las variaciones que tienen los ángulos durante el proceso de corte.

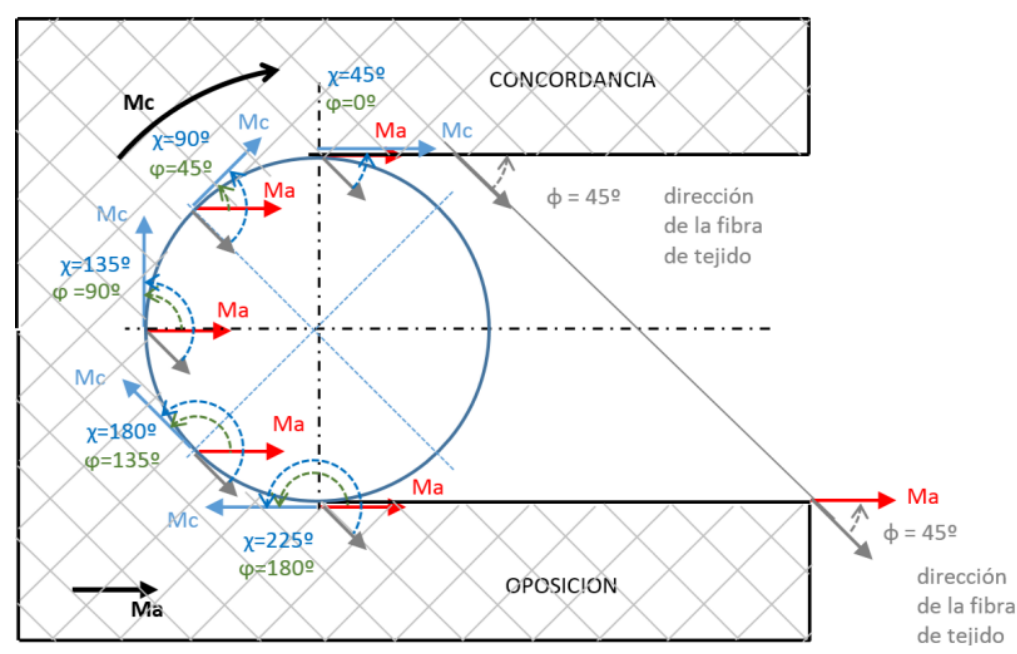

(a) $\phi=45^{\circ}$ 


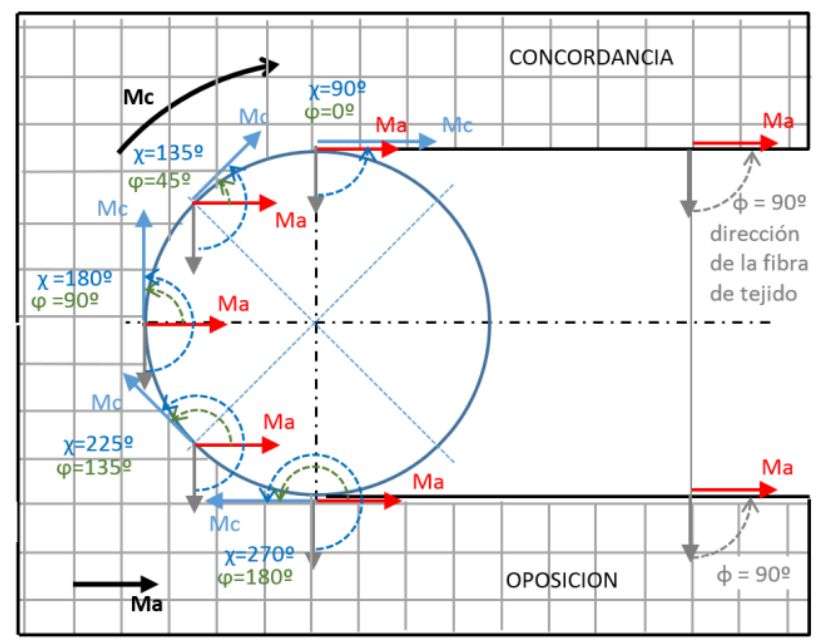

(b) $\phi=90^{\circ}$

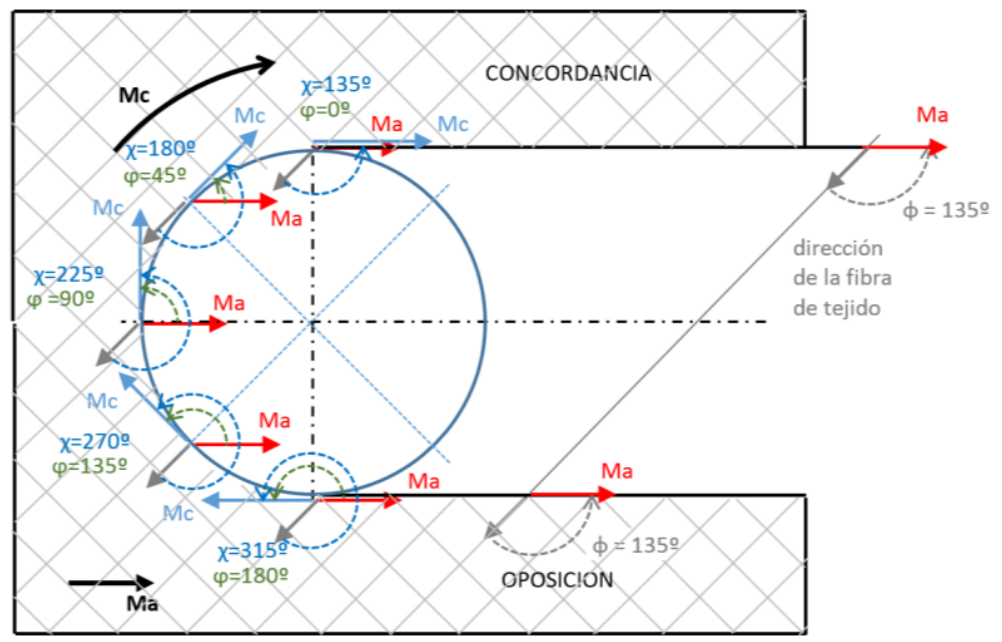

(c) $\phi=135^{\circ}$

Figura 4.4. Ángulos de referencia en el mecanizado de una ranura.

\subsubsection{ORIENTACIÓN DE LA TRAYECTORIA DE CORTE RESPECTO AL TEJIDO: EFECTO EN EL DELAMINADO TIPO II}

En el proceso de contorneado de una pieza, en función de su geometría exterior y de la orientación de las fibras en las capas externas de la pieza se 
puede producir delaminado. Se ha analizado este resultado para el delaminado tipo II que es el que resulta más evidente en este tipo de procesos de mecanizado.

Atendiendo a su apariencia, el delaminado tipo II observado en los experimentos se puede clasificar como delaminado uniforme, delaminado con efecto onda y delaminado aleatorio.

\subsubsection{Delaminado uniforme}

Se dice que el delaminado es uniforme cuando todas las mechas de tejido tienen un delaminado con la misma apariencia, similar en longitud invadida, orientación y superficie delaminada.

Este delaminado uniforme se observa cuando el tejido no tiene deformaciones y está correctamente orientado con respecto al movimiento de avance, es decir, el ángulo de orientación de fibra $(\phi)$ es conocido y coincide con una de las orientaciones principales del tejido, para valores del ángulo de orientación de la fibra $(\phi)$ de $45^{\circ}, 90^{\circ}$ y $135^{\circ}$ (Figura 4.5). En esta situación se puede predecir el aspecto que tendrá el delaminado del borde de la pieza.

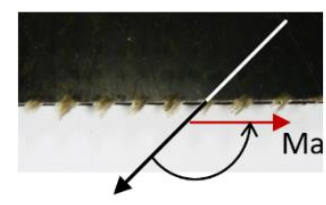

$\phi=135^{\circ}$

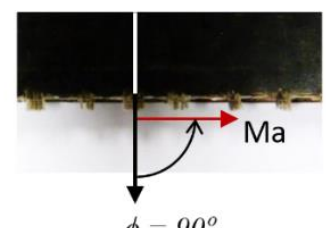

$\phi=90^{\circ}$

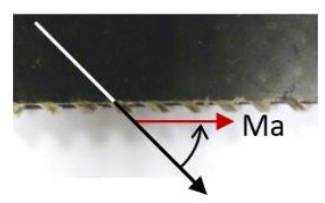

$\phi=45^{\circ}$

Figura 4.5. Delaminado tipo II uniforme en orientaciones principales.

El delaminado se produce porque los filos de corte de la fresa doblan las mechas de tejido sin llegar a cortarlas. Con un ángulo de orientación de fibra $(\phi)$ de $45^{\circ}$ ó $135^{\circ}$, el borde mecanizado presenta en toda su longitud, el mismo aspecto con fibras inclinadas con la misma dirección del tejido. Estas fibras sobresalen del borde de la pieza, una por cada mecha que conforma el tejido (Figura 4.6 (a) y (c)).

Con un ángulo de orientación de fibra $(\phi)$ de $90^{\circ}$, la trama del tejido sirve de soporte de forma alterna a las mechas de urdimbre. Por ello, las mechas de urdimbre que van por debajo de la trama suelen presentar un corte limpio, sin provocar delaminado o siendo de menor influencia, mientras que las que van por encima de la trama, se doblan y provocan el defecto que se observa en la Figura 4.6 (b). 


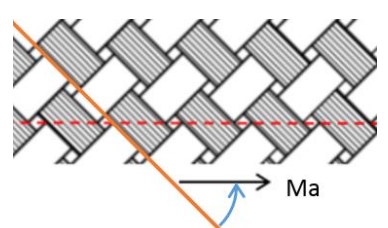

$\phi=x=459$
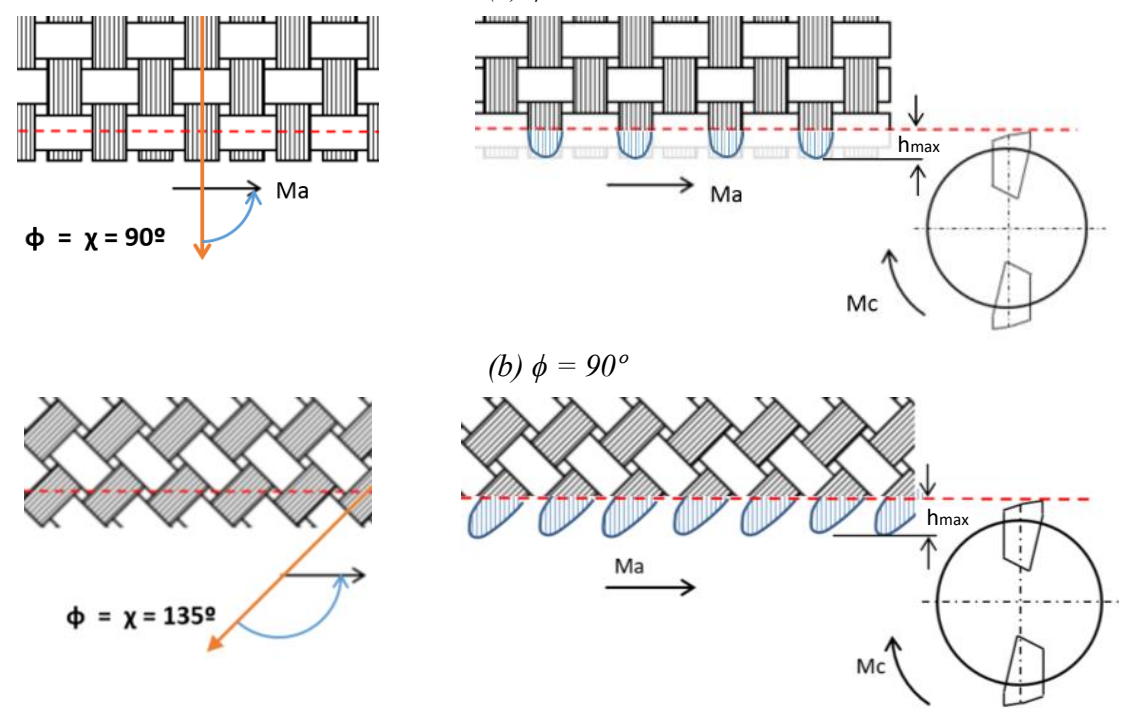

(c) $\phi=135^{\circ}$

(a) $\phi=45^{\circ}$

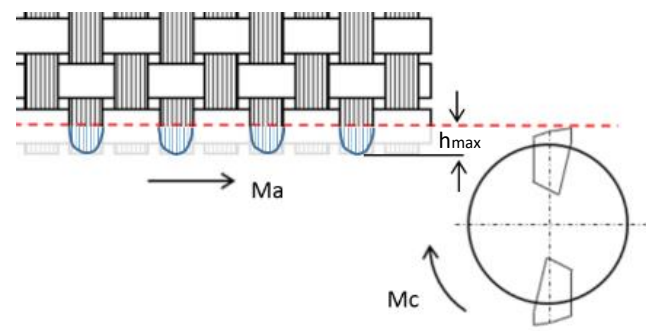

(b) $\phi=90^{\circ}$

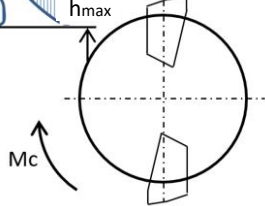


Se ha definido el ángulo de inclinación del tejido $(\rho)$, como el ángulo formado desde la dirección del movimiento de corte hasta la dirección de la trama del tejido, en sentido antihorario (Figura 4.8).

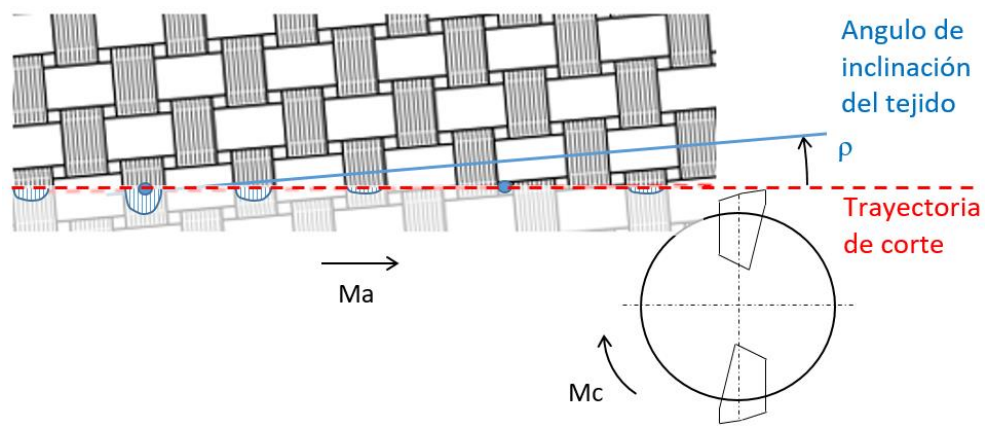

Figura 4.8. Angulo de inclinación del tejido ( $\rho)$.

Debido a esta inclinación del tejido respecto a la trayectoria de corte, la herramienta pasa de una mecha a la contigua, atravesando el cruce entre las mechas que conforman el tejido. El espacio entre mechas, debido al efecto del entrecruzamiento del tejido, tiene abundancia de resina superficial que sujeta bien el refuerzo, por lo que, en la mayoría de las ocasiones, el corte que se produce es limpio. En esta zona el delaminado es mínimo o incluso nulo. Sin embargo, conforme la trayectoria de corte se aleja del cruce entre dos mechas y se acerca al centro de la mecha, el delaminado aumenta progresivamente hasta un valor máximo, donde el delaminado tiene un aspecto denso, que corresponde a la zona de la pieza donde hay menos resina superficial, para a continuación volver a disminuir hasta llegar al siguiente cruce.

El efecto de esta inclinación del tejido en el mecanizado produce lo que denominamos efecto onda del delaminado (Figura 4.9).

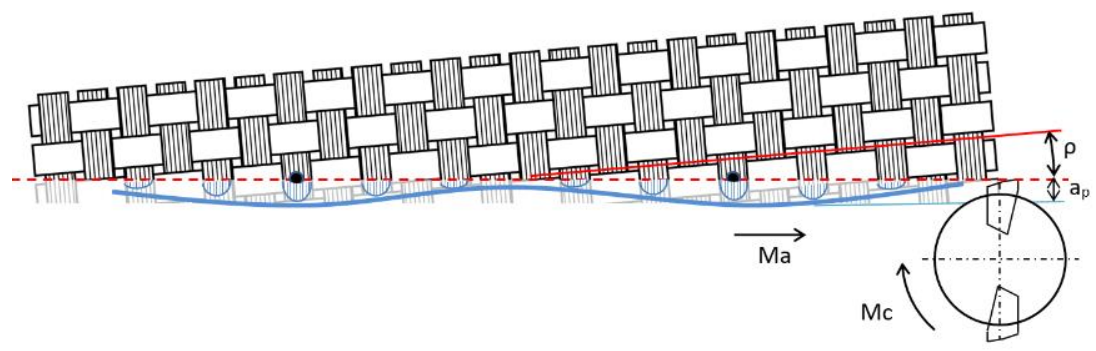

Figura 4.9. Delaminado tipo II con efecto onda. 


\subsubsection{Delaminado aleatorio}

En ocasiones el delaminado presenta cambios de orientación o un aspecto completamente irregular en el borde mecanizado (Figura 4.10). Este aspecto irregular se produce en dos situaciones: cuando la trayectoria de corte de la herramienta está ligeramente inclinada con un ángulo $(\rho)$ respecto a un tejido con orientación de $45^{\circ}-135^{\circ}$ o cuando el tejido presenta deformaciones debido al proceso de fabricación. En estos dos casos, la orientación de la fibra varía continuamente respecto a la trayectoria de corte de la herramienta, lo que produce un efecto de delaminado aleatorio [105].

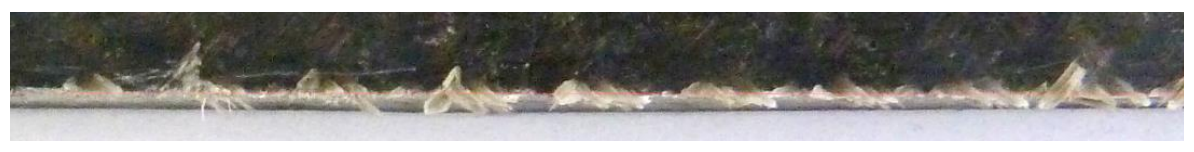

Figura 4.10. Delaminado irregular.

En la Figura 4.11 se observan las mechas de tejido con una orientación de fibra $(\phi)$ de $135^{\circ}$, ligeramente inclinadas con respecto a la trayectoria de corte, el ángulo $\rho$. Estas mechas con la misma orientación de tejido producen el delaminado en la misma dirección, pero no del mismo tamaño, ya que en función del punto en el que incida la herramienta sobre la mecha, el delaminado también varía.

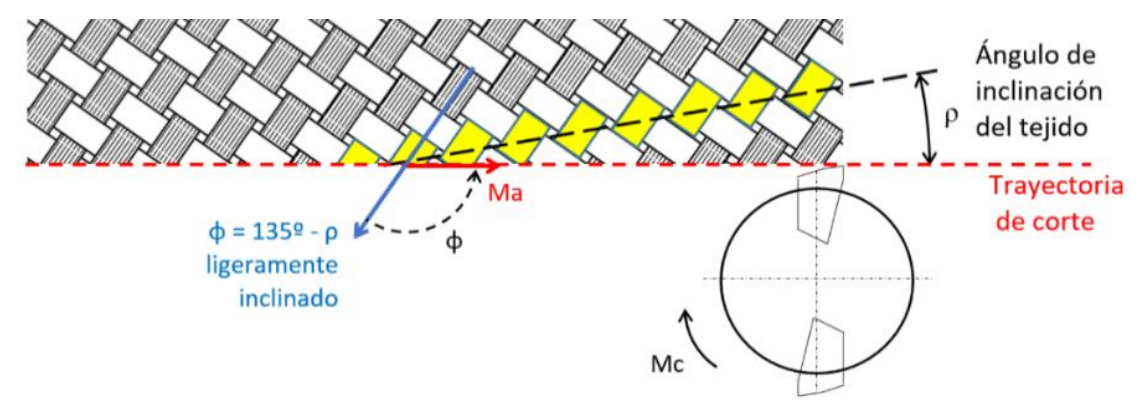

Figura 4.11. Tejido ligeramente inclinado $\phi=135^{\circ}-\rho$.

Por otro lado, al tratarse de un tejido bidireccional, dos mechas contiguas tienen una diferencia de $90^{\circ}$. El delaminado que se produce en cada punto, corresponde a la orientación de la mecha que se está cortando en ese instante (Figura 4.12), esto produce para esta orientación de tejido un efecto continuo de delaminado cruzado. 


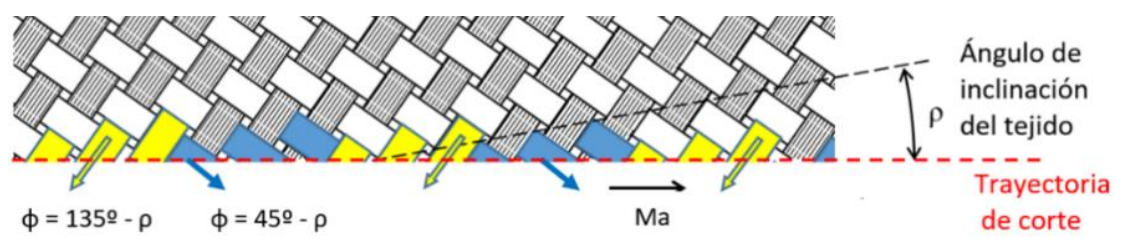

Figura 4.12. Dirección de las mechas delaminadas en un tejido bidireccional.

En la Figura 4.13 se ha representado el caso de un tejido con ángulo de inclinación $\rho=10^{\circ}$ respecto a la dirección principal de $45^{\circ}$, es decir, un ángulo de orientación de fibra $(\phi)$ de $125^{\circ}$ y de $35^{\circ}$ respectivamente respecto a la trayectoria de corte. En esta situación se distinguen áreas con y sin delaminado en función del punto donde la hebra es cortada y de su orientación, provocando un aspecto delaminado irregular.

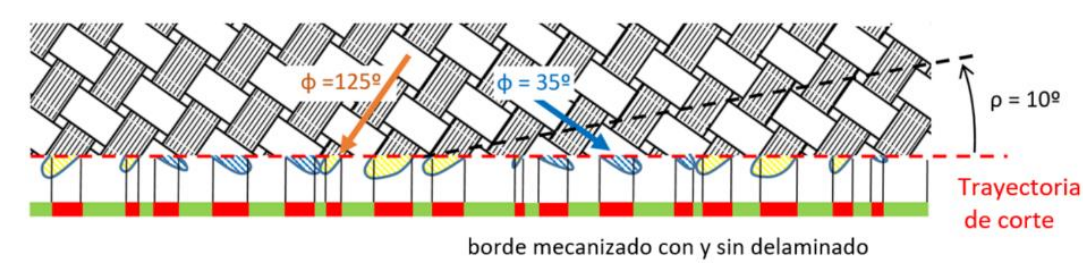

\begin{tabular}{|c|c|c|c|}
\hline Zona CON delaminado & & Delaminado tipo II con orientación 350 & MाMाप्य \\
\hline Zona SIN delaminado & & Delaminado tipo II con orientación 1250 & $\mathrm{WWM}$ \\
\hline
\end{tabular}

Figura 4.13. Delaminado tipo II aleatorio.

\subsubsection{PARÁMETRO Xd}

El delaminado producido en la pieza durante el mecanizado depende directamente del valor de Xd en cada punto. El parámetro Xd se define como la distancia del punto donde incide la herramienta sobre la pieza a mecanizar, hasta el cruce de mechas de tejido más próximo. Los valores que puede tomar este parámetro dependerán de la orientación de la fibra del tejido y el ancho de la mecha, así como del ángulo de inclinación del tejido con la trayectoria de corte. Este parámetro puede tomar un valor constante, seguir un patrón de variación uniforme o puede ser totalmente aleatorio. 


\subsubsection{Valor constante de Xd}

Cuando el tejido tiene una orientación $(\phi)$ de $0^{\circ}-90^{\circ}$, el movimiento de avance coincide con la dirección de la trama del tejido. En esta situación, el valor de Xd es constante y coincide con la distancia desde el punto donde incide la herramienta hasta el cruce con la siguiente mecha (Figura 4.14). En este caso, el valor de $\mathrm{Xd}$ es constante y puede llegar teóricamente como máximo hasta el valor del tamaño de la mecha.

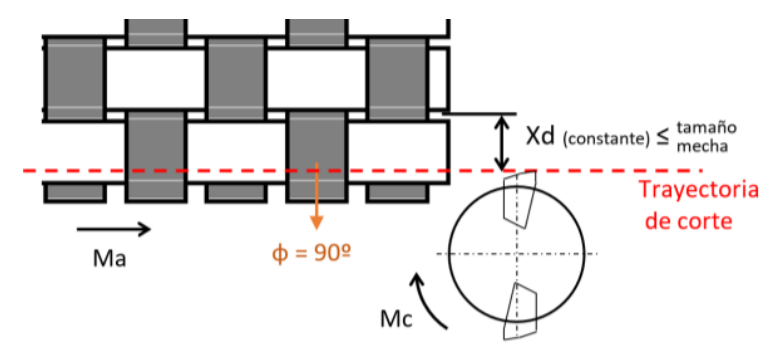

Figura 4.14. Parámetro Xd con $\phi=90^{\circ}$.

\subsubsection{Patrón de variación uniforme para $X d$}

Hay algunas situaciones en las que el parámetro $\mathrm{Xd}$ no tiene un valor constante, pero sigue un patrón de variación uniforme y predecible. Estos casos son los siguientes:

- tejido con un ángulo de orientación $\phi$ de $45^{\circ}-135^{\circ}$.

- tejido con un ángulo de orientación $\phi$ de $0^{\circ}-90^{\circ}$, pero con un ligero ángulo de inclinación $(\rho)$ entre la dirección principal de la trama del tejido y la trayectoria de corte.

En el primer caso, la Figura 4.15 muestra la variación de Xd entre dos mechas contiguas a $90^{\circ}$ (tejido bidireccional), sobre las direcciones principales del tejido de $45^{\circ}-135^{\circ}$. En estos casos el parámetro Xd varía desde cero, hasta el valor de media diagonal o hasta el valor de la diagonal entera de la mecha, dependiendo del punto donde cruce la trayectoria de corte de la herramienta sobre la mecha de tejido. Si la trayectoria de la herramienta cruza entre dos mechas con el mismo ángulo de orientación de fibra $(\phi)$, el parámetro Xd varía siempre entre $0 \leq \mathrm{Xd} \leq 1 / 2$ diagonal. Por el contrario, si el cruce se produce entre mechas a $90^{\circ}$, el parámetro Xd llega a alcanzar el valor de la diagonal entera.

En la Figura 4.16 se muestra un esquema del valor real que toma $\mathrm{Xd}$ en cada punto. Cuando la trayectoria de corte pasa por el espacio de separación 
entre dos mechas contiguas el parámetro Xd tiene valor cero, aumentando o disminuyendo conforme se aleja o acerca al siguiente cruce de mechas de tejido.

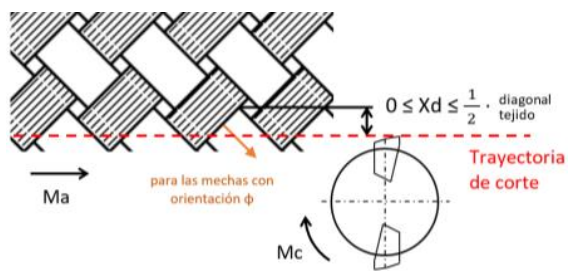

(a) $\phi$

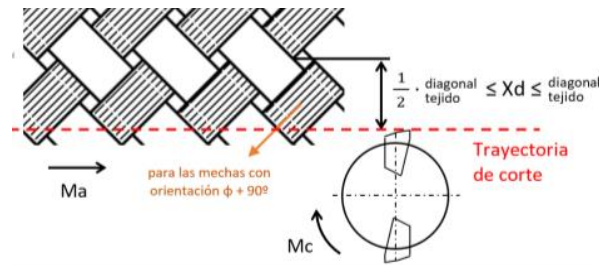

(b) $\phi+90^{\circ}$

Figura 4.15. Variación de Xd entre mechas contiguas a $90^{\circ}$.

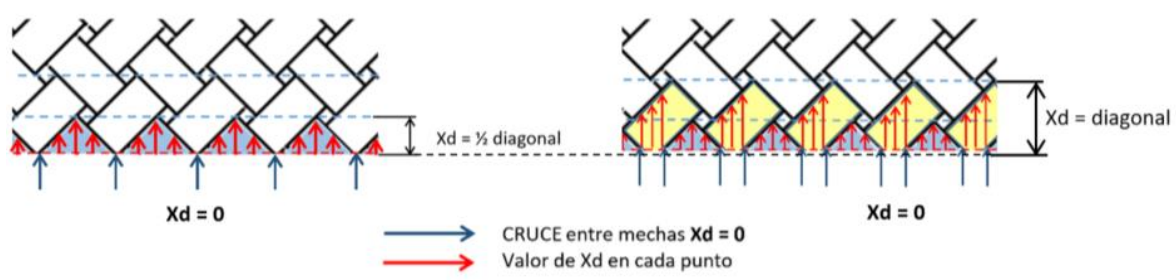

(a) $0 \leq X d \leq 1 / 2$ diagonal

(b) $0 \leq X d \leq$ diagonal

Figura 4.16. Variación de Xd con $\phi=45^{\circ}-135^{\circ}$.

En el segundo caso, la trayectoria de mecanizado está ligeramente inclinada ( $\rho$ ) con respecto a la trama del tejido, lo que provoca el efecto onda del delaminado, explicado en el apartado anterior. El Xd aumenta su valor progresivamente hasta que llega al cruce y cambia de mecha, donde empieza de nuevo con valor cero hasta alcanzar el valor de la mecha (Figura 4.17).

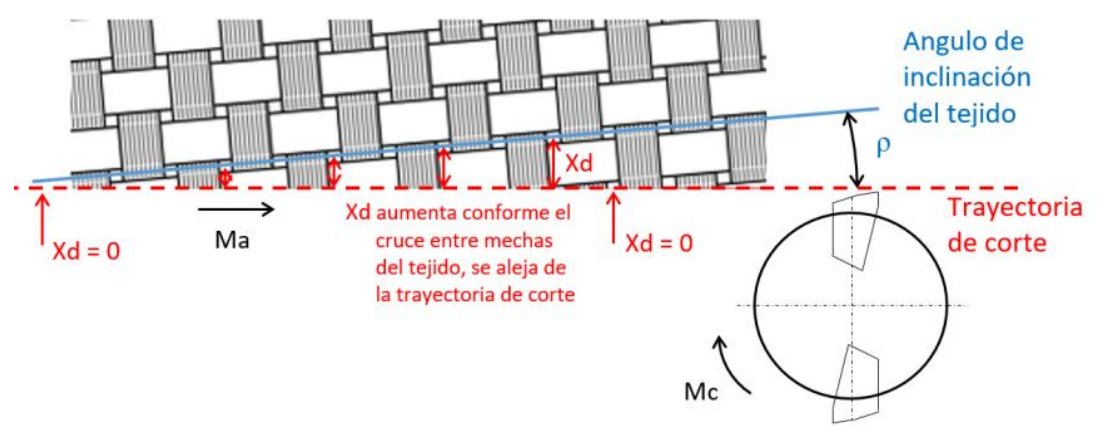

Figura 4.17. Trayectoria de corte ligeramente inclinada ( $\rho)$. 
En esta situación, la línea de corte presenta a lo largo de su longitud zonas con y sin delaminado. El delaminado se reduce a cero cuando la herramienta pasa en el espacio vacío de tejido entre dos mechas, donde el parámetro $\mathrm{Xd}=$ 0 ó $\mathrm{Xd}=$ tamaño de mecha. El delaminado alcanza su valor máximo cuando la trayectoria de la herramienta cruza por el centro de una mecha de tejido, con $\mathrm{Xd}=1 / 2 \cdot$ mecha.

La Figura 4.18 muestra conjuntamente la variación de Xd y el delaminado producido a lo largo del borde de la pieza. En ella se marca la línea central de la mecha, que es la zona de la pieza donde la resina es menos abundante y es más crítica para sufrir delaminado.

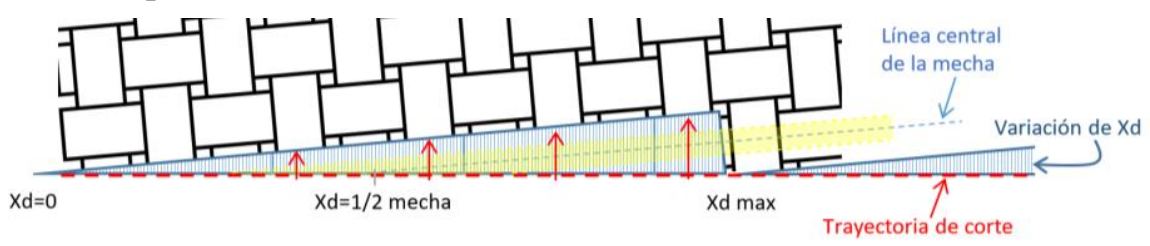

(a) $\mathrm{Xd}$

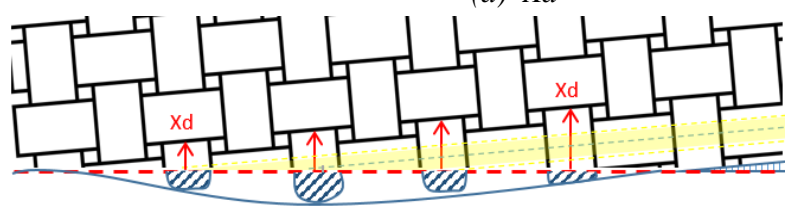

(b) Delaminado

Figura 4.18. Variación de Xd y del delaminado.

\subsubsection{Valor aleatorio de $\mathrm{Xd}$}

El parámetro Xd toma valores aleatorios en los siguientes casos:

- Cuando existe una ligera inclinación entre la trayectoria de corte y la dirección principal de $45^{\circ}-135^{\circ}$ del tejido (Figura 4.19). El valor de Xd varía siguiendo la forma del entrecruzamiento del tejido y también varía dentro de la mecha, provocando esa misma variación en el delaminado observado.
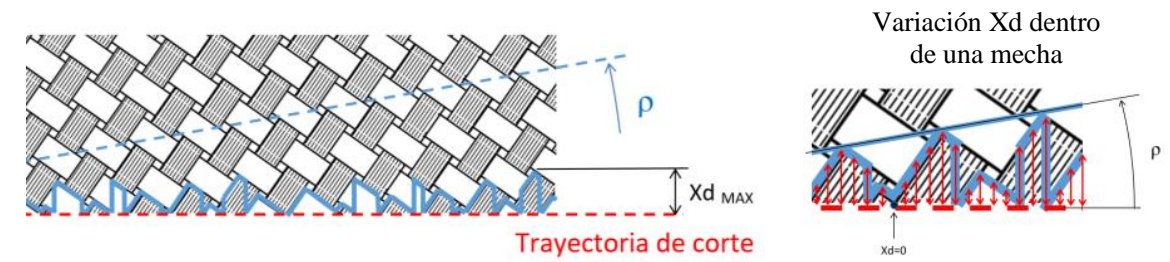

Figura 4.19. Variación de Xd para tejido $45^{\circ}-135^{\circ}$ inclinado ligeramente $\rho$. 
- Cuando el tejido presenta deformaciones y la orientación de las mechas no forma ningún ángulo conocido o no coincide, con la trayectoria de mecanizado. En este caso el Xd es aleatorio por naturaleza, y, en consecuencia, el delaminado producido también es completamente aleatorio. Esto puede ser debido a que el tejido se ha movido durante la inyección de la resina en el proceso de fabricación del laminado.

\subsection{ANÁLISIS PRELIMINAR DEL DELAMINADO}

En primer lugar, se va a realizar un análisis visual y cualitativo de los diferentes laminados obtenidos en cada uno de los experimentos, para los diferentes tiempos de mecanizado ( $\mathrm{Tc}=30,50,60,70$ y $80 \mathrm{~min}$ ). De esta forma se establecerá la mejor forma de medirlo y se descartarán, para un estudio posterior cuantitativo, aquellos experimentos que no presenten delaminado o cuando éste sea despreciable.

Tras realizar este examen visual, lo primero que se hace evidente es que las piezas que tienen un volumen de fibra $\mathrm{Fv}=40 \%$ no presentan delaminado, por lo que no serán evaluadas. Sin embargo, para piezas fabricadas con volumen de fibra alto $(\mathrm{Fv}=60 \%)$ siempre aparece este efecto en el borde (Figura 4.20).

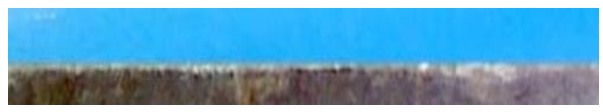

(a) Fv $40 \%$ sin delaminado

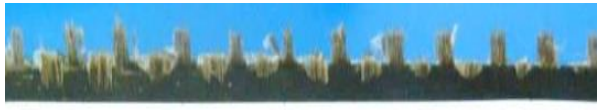

(b) Fv $60 \%$ con delaminado

Figura 4.20. Aspecto de la superficie mecanizada.

En las piezas donde se aprecia delaminado predomina el tipo II frente al tipo I, y el tipo III se presenta en muy pocos experimentos.

\subsubsection{VOLUMEN DE FIBRA}

La influencia del volumen de fibra en la aparición del delaminado, se debe principalmente al efecto de soporte de la capa de resina superficial que conforma la lámina de material compuesto. Tras el fresado, esto se aprecia en el borde de las piezas. En las zonas donde no hay suficiente capa de resina que sujete las mechas de tejido, la fuerza de la herramienta durante el proceso de corte dobla las hebras de fibra del tejido en vez de cortarlas y se produce delaminado. Si sobre la capa más exterior de tejido hay suficiente resina se obtiene un corte más limpio (Figura 4.21). 


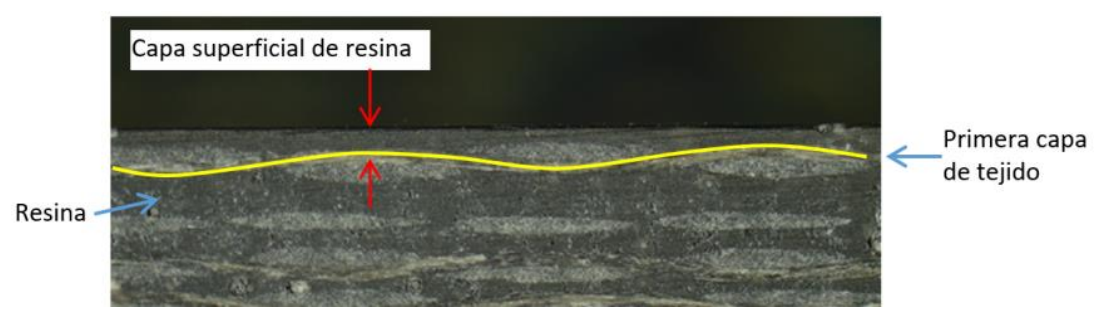

(a) $F v 40 \%$

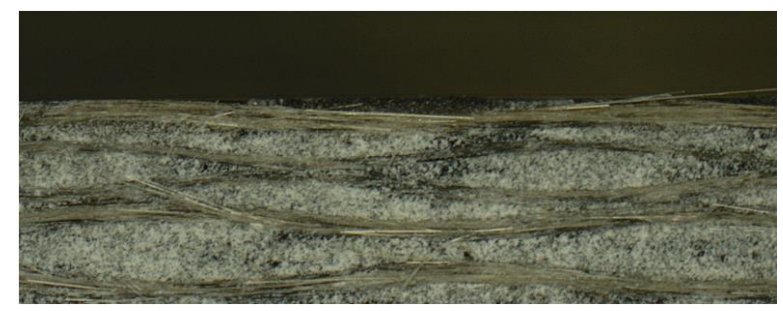

(b) $F v 60 \%$

Figura 4.21. Detalle de la capa de resina superficial.

Este efecto se puede explicar si se observa la disposición del tejido en el espesor mecanizado (Figura 4.22). El entrecruzamiento de la trama y la urdimbre en el tejido provoca la ondulación de las hebras en dos direcciones, y una distribución irregular de la resina en torno al tejido de refuerzo.

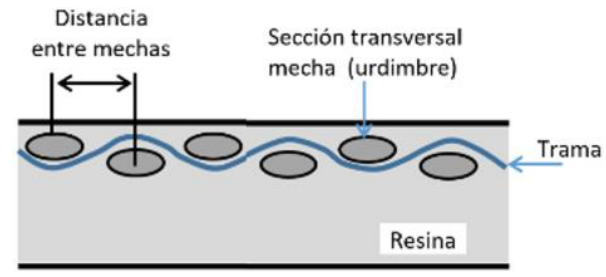

(a) Disposición de las mechas de tejido

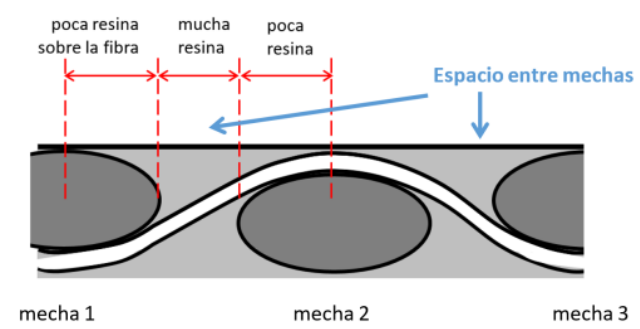

(b) Detalle de la resina superficial

Figura 4.22. Disposición de las mechas de tejido en la capa superficial de la pieza. 
Tras el curado de la pieza, las mechas de tejido quedan fijas por el endurecimiento de la resina, formando ondulaciones por el paso sobre y bajo la hebra contigua. La capa superficial de resina que soporta el tejido es mayor en el espacio entre mechas y se reduce en el centro de la mecha, reduciendo también su resistencia cuando actúa la fuerza de corte. El centro de la mecha es, por lo tanto, el punto más débil y más propenso a sufrir delaminado.

En la Figura 4.23 se observa el detalle de una pieza defectuosa que tiene una distribución inadecuada de las fibras. En la pieza sólo aparece delaminado en las zonas donde el tejido toca directamente con el borde inferior y/o superior de la pieza, donde hay casi ausencia de resina. Sin embargo, las zonas donde aparece una acumulación importante de resina, presentan un corte limpio y libre de delaminado.

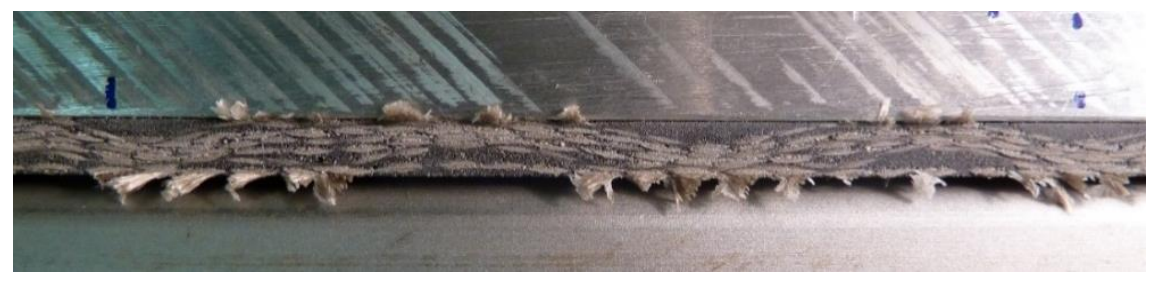

Figura 4.23. Delaminado con distribución irregular de la fibra y la resina.

\subsubsection{EXPERIMENTOS CON DELAMINADO}

En las piezas que tienen un volumen de fibra elevado $(\mathrm{Fv}=60 \%)$, se ha realizado una valoración cualitativa sobre sus caras exteriores, la cara superior (SUP) y la cara inferior (INF) para cada uno de los tiempos evaluados en los diferentes experimentos $(\mathrm{Tc}=30,50,60,70$ y 80 minutos) (Tabla 4.1). Para ello se han observado todas las piezas en global, evaluando los tipos de delaminado que aparecen en ellas.

Tabla 4.1. Tipos de delaminado observados.

\begin{tabular}{|c|c|c|c|c|c|c|}
\hline \multirow[b]{2}{*}{ Exp } & \multirow[b]{2}{*}{ Condiciones } & \multirow[b]{2}{*}{$\begin{array}{l}\text { cara de } \\
\text { la pieza }\end{array}$} & \multicolumn{4}{|c|}{ presenta zonas con: } \\
\hline & & & $\begin{array}{c}\text { Solo } \\
\text { Tipo I }\end{array}$ & $\begin{array}{c}\text { Solo } \\
\text { Tipo II }\end{array}$ & Tipo I/II & Tipo III \\
\hline \multirow[t]{2}{*}{ E04 } & Vc $300 ;$ Fz 0.4 & SUP & $\mathrm{NO}$ & SI & SI & poco \\
\hline & $a_{p} 1.5 ; F o 45^{\circ}$ & INF & $\mathrm{NO}$ & SI & SI & NO \\
\hline \multirow[t]{2}{*}{ E05 } & Vc $470 ;$ Fz 0.1 & SUP & $\mathrm{NO}$ & SI & SI & poco \\
\hline & $a_{p} 0.5 ; F_{0} 90^{\circ}$ & INF & $\mathrm{NO}$ & SI & SI & $\mathrm{NO}$ \\
\hline
\end{tabular}




\begin{tabular}{|c|c|c|c|c|c|c|}
\hline \multirow[b]{2}{*}{ Exp } & \multirow[b]{2}{*}{ Condiciones } & \multirow[b]{2}{*}{$\begin{array}{l}\text { cara de } \\
\text { la pieza }\end{array}$} & \multicolumn{4}{|c|}{ presenta zonas con: } \\
\hline & & & $\begin{array}{l}\text { Solo } \\
\text { Tipo I }\end{array}$ & $\begin{array}{c}\text { Solo } \\
\text { Tipo II }\end{array}$ & Tipo I/II & Tipo III \\
\hline \multirow[t]{2}{*}{ E07 } & Vc $300 ;$ Fz 0.1 & SUP & poco & poco & NO & NO \\
\hline & $\mathrm{ap}_{\mathrm{p}} 0.5 ; \mathrm{Fo} 45^{\circ}$ & INF & poco & SI & NO & NO \\
\hline \multirow[t]{2}{*}{ E08 } & Vc $470 ; \mathrm{Fz} 0.4$ & SUP & SI & SI & SI & NO \\
\hline & $\mathrm{a}_{\mathrm{p}} 0.5 ; \mathrm{Fo} 45^{\circ}$ & INF & SI & SI & NO & NO \\
\hline \multirow[t]{2}{*}{ E11 } & Vc $300 ;$ Fz 0.1 & SUP & NO & SI & SI & poco \\
\hline & ap $1.5 ;$ Fo $90^{\circ}$ & INF & NO & SI & NO & NO \\
\hline \multirow[t]{2}{*}{ E12 } & Vc $300 ;$ Fz 0.4 & SUP & SI & SI & SI & poco \\
\hline & $\mathrm{a}_{\mathrm{p}} 0.5 ; \mathrm{Fo} 90^{\circ}$ & INF & NO & SI & SI & NO \\
\hline \multirow[t]{2}{*}{ E14 } & Vc $470 ; \mathrm{Fz} 0.4$ & SUP & NO & SI & SI & SI \\
\hline & ap $1.5 ;$ Fo $90^{\circ}$ & INF & SI & SI & SI & NO \\
\hline \multirow[t]{2}{*}{ E16 } & Vc $470 ;$ Fz 0.1 & SUP & SI & SI & SI & NO \\
\hline & $a_{p} 1.5 ; F o 45^{\circ}$ & INF & SI & SI & NO & NO \\
\hline
\end{tabular}

Tras este primer análisis se pueden extraer las siguientes conclusiones generales:

- El efecto del delaminado sobre la cara superior de la pieza es mayor que sobre la cara inferior.

- El delaminado tipo II es, con diferencia, el más abundante de todos los tipos de delaminado evaluados.

- En los experimentos con profundidad de pasada pequeña, $\mathrm{a}_{\mathrm{p}}=0,5 \mathrm{~mm}$ se aprecia un delaminado menor que en los experimentos con profundidad de pasada mayor, $\mathrm{a}_{\mathrm{p}}=1,5 \mathrm{~mm}$.

- El delaminado tipo I coexiste muchas veces con el tipo II, provocando un delaminado combinado Tipo I/II que acentúa el defecto en el borde de la pieza.

- El delaminado tipo III tiene poca o nula influencia en todos los experimentos salvo en el E14 en la cara superior.

- No se aprecian variaciones ni tendencias claras para el delaminado observado cuando aumenta el tiempo de corte, y, por consiguiente, con el aumento del desgaste de la herramienta.

A partir de estas observaciones, para evaluar el delaminado de forma cuantitativa, se utilizarán las muestras obtenidas de la cara superior de las piezas, por representar un límite superior y permitir acotar el defecto. 


\subsubsection{TRATAMIENTO DE LAS ZONAS CON DELAMINADO}

En este apartado se analizará el borde de la pieza que presenta delaminado. Cada laminado utilizado en los experimentos tiene una longitud total $(\mathrm{Lt}=240$ $\mathrm{mm}$ ) en la que se ha medido, para cada experimento y tiempo de mecanizado, qué longitud presenta delaminado y de qué tipo. Para medir la longitud se distinguen varias zonas en el borde mecanizado y se agrupan de la siguiente forma según el tipo de delaminado. La longitud total para el delaminado tipo I, se calcula como la suma de la zona con delaminado tipo I (1_tipoI) y la zona sin delaminado tipo I, que corresponde al resto del borde de la pieza.

Para el delaminado tipo II, la longitud de la pieza es el resultado de una zona con corte limpio y otras zonas con borde delaminado. La zona con corte limpio es la zona del borde mecanizado sin delaminado tipo II y III (1_SIN_tipoIIyIII), sin hilos que sobresalen del borde mecanizado (ecuación 4.2).

$$
\mathrm{Lt}=\sum\left(1_{\_} \text {CON_tipo IIyIII }\right)+\sum\left(1_{\_} \text {SIN_tipo IIyIII }\right)=240 \mathrm{~mm} \quad \text { (ecuación 4.2) }
$$

La zona con borde delaminado, es la zona que presenta delaminado II y/o III (1_Con_tipoIIyII). Esta longitud a su vez incluye la mecha delaminada $\left(l_{i}\right)$ y el espacio libre entre mechas de tejido (l $1_{\text {espacio_libre }}$ ) (ecuación 4.3).

$$
\begin{aligned}
\text { Zona con borde delaminado } & =\sum\left(1_{-}\right. \text {Con_tipo IIyIII) } \\
& =\sum 1_{i}+\sum \text { lespacio libre } \quad \text { (ecuación 4.3) }
\end{aligned}
$$

La apariencia de la zona con borde delaminado depende del tipo de entrecruzamiento del tejido por lo que el valor de estas longitudes debe adecuarse al tipo de tejido. En la toma de datos, independientemente de cómo se presente a lo largo del borde del laminado, se han agrupado en un solo valor como la suma de las longitudes que aparecen de cada tipo (Figura 4.24).

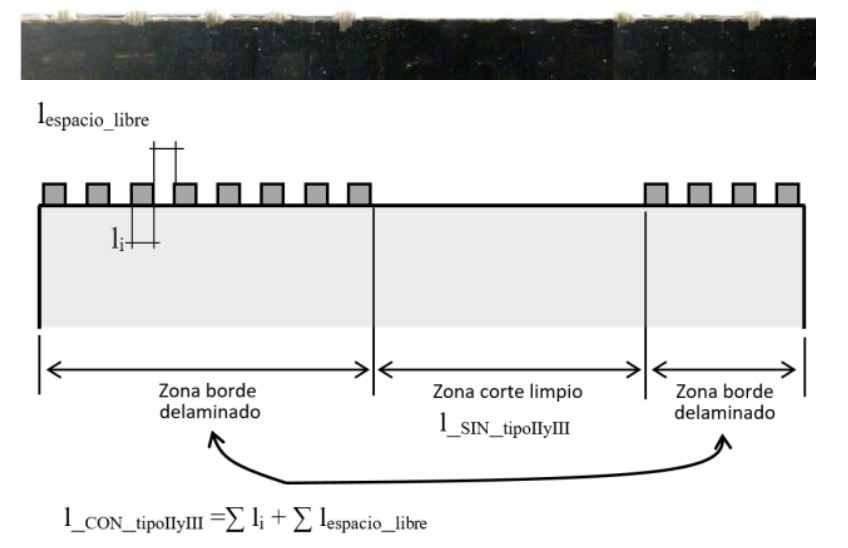

(a) Ejemplo medida longitud delaminada Tipo II en tejido $F o=0^{\circ}-90^{\circ}$ 


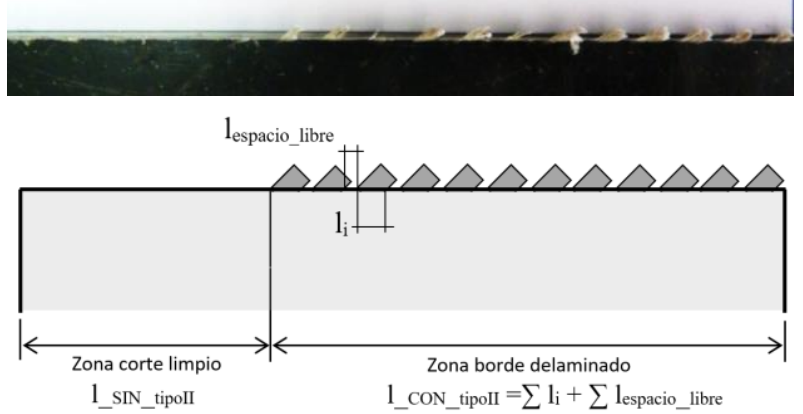

(b) Ejemplo medida longitud delaminada Tipo II en tejido Fo $=45^{\circ}-135^{\circ}$

Figura 4.24. Zona borde delaminado y zona corte limpio en tipo II.

En la Tabla 4.2, se presenta la longitud de la pieza repartida entre zona con delaminado y la zona con corte limpio, observando para cada experimento si presenta o no zonas sin delaminado, además del porcentaje del total de la pieza que presenta delaminado. Se ha distinguido entre el delaminado que afecta a la superficie de la pieza (tipo I) y el que sobresale de la pieza (tipos II+III). Si para un determinado experimento se suman los milímetros de la pieza que tiene delaminados del tipo I, tipos II+III y zona sin delaminado, se observa que no suman $240 \mathrm{~mm}$. Esto es debido a que, en algunas zonas, existen conjuntamente delaminados tipo I y tipos II+III, pero los tipos II y III junto con la zona limpia, sí suman la longitud total de $240 \mathrm{~mm}$. El delaminado se ha medido sólo para tiempos de corte $\mathrm{Tc}=30,50,60,70$ y $80 \mathrm{~min}$, considerando ya que para 5 minutos su valor es prácticamente despreciable.

Tabla 4.2. Zonas con distintos tipos de delaminado

\begin{tabular}{|c|c|c|c|c|c|c|c|c|c|}
\hline \multirow[t]{4}{*}{ Exp } & \multirow[t]{3}{*}{$\mathrm{Tc}$} & \multicolumn{2}{|c|}{$\begin{array}{c}\text { LONGITUD } \\
\text { Zona delaminada }\end{array}$} & \multirow{4}{*}{$\begin{array}{c}\text { Zona } \\
\text { limpia } \\
1_{\mathrm{SIN}} \\
\text { tipoIIyIII } \\
\mathrm{mm}\end{array}$} & \multirow{4}{*}{$\begin{array}{l}\text { Longitud } \\
\text { total } \\
\mathrm{Lt} \\
\mathrm{mm}\end{array}$} & \multicolumn{2}{|c|}{$\begin{array}{l}\text { PORCENTAJE } \\
\text { Zona delaminada }\end{array}$} & \multirow{4}{*}{$\begin{array}{c}\text { Zona } \\
\text { limpia } \\
1_{\mathrm{SIN}} \\
\text { tipoIIyIII } \\
\%\end{array}$} & \multirow[t]{4}{*}{ Observaciones } \\
\hline & & Tipo I & Tipo II+III & & & Tipo I & Tipo II+III & & \\
\hline & & $1_{\text {_tipoI }}$ & $\underset{\text { tipoIIyIII }}{\text { 1_CON }}$ & & & $1_{-}$tipol & $\underbrace{\text { 1_CON }}_{\text {tipoIIyIII }}$ & & \\
\hline & $\min$ & $\mathrm{mm}$ & $\mathrm{mm}$ & & & $\%$ & $\%$ & & \\
\hline \multirow[t]{5}{*}{ E04 } & 30 & 240 & 210 & 30 & & 100 & 87.5 & 12.5 & \\
\hline & 50 & 80 & 240 & 0 & & 33.3 & 100 & 0 & sin zona limpia \\
\hline & 60 & 15 & 240 & 0 & 240 & 6.3 & 100 & 0 & sin zona limpia \\
\hline & 70 & 25 & 240 & 0 & & 10.4 & 100 & 0 & sin zona limpia \\
\hline & 80 & 120 & 240 & 0 & & 50 & 100 & 0 & sin zona limpia \\
\hline \multirow[t]{5}{*}{ E05 } & 30 & 132 & 140 & 100 & & 55 & 58.3 & 41.7 & \\
\hline & 50 & 145 & 100 & 140 & & 60.4 & 41.7 & 58.3 & \\
\hline & 60 & 150 & 110 & 130 & 240 & 62.5 & 45.8 & 54.2 & \\
\hline & 70 & 83 & 123 & 117 & & 34.6 & 51.3 & 48.8 & \\
\hline & 80 & 95 & 24 & 216 & & 39.6 & 10 & 90 & \\
\hline
\end{tabular}




\begin{tabular}{|c|c|c|c|c|c|c|c|c|c|}
\hline \multirow[t]{4}{*}{ Exp } & \multirow[t]{3}{*}{$\mathrm{Tc}$} & \multicolumn{2}{|c|}{$\begin{array}{c}\text { LONGITUD } \\
\text { Zona delaminada }\end{array}$} & \multirow{4}{*}{$\begin{array}{c}\text { Zona } \\
\text { limpia } \\
\text { 1_sIN_ } \\
\text { tipoIIyIII } \\
\text { mm }\end{array}$} & \multirow{4}{*}{$\begin{array}{c}\text { Longitud } \\
\text { total } \\
\mathrm{Lt} \\
\mathrm{mm}\end{array}$} & \multicolumn{2}{|c|}{$\begin{array}{l}\text { PORCENTAJE } \\
\text { Zona delaminada }\end{array}$} & \multirow{4}{*}{$\begin{array}{c}\text { Zona } \\
\text { limpia } \\
\text { 1_SIN_ } \\
\text { tipoIIIII } \\
\%\end{array}$} & \multirow[t]{4}{*}{ Observaciones } \\
\hline & & Tipo I & Tipo II+III & & & Tipo I & Tipo II+III & & \\
\hline & & $1_{- \text {tipol }}$ & $\begin{array}{l}\text { 1_CON_} \\
\text { tipoIIyIII }\end{array}$ & & & $1_{\text {_tipoI }}$ & 1_CON_- $_{\text {tipoIIIIII }}$ & & \\
\hline & $\min$ & $\mathrm{mm}$ & $\mathrm{mm}$ & & & $\%$ & $\%$ & & \\
\hline \multirow[t]{5}{*}{ E07 } & 30 & 86 & 60 & 180 & & 35.8 & 25 & 75 & \\
\hline & 50 & 161 & 30 & 210 & & 67.1 & 12.5 & 87.5 & \\
\hline & 60 & 140 & 0 & 240 & 240 & 58.3 & 0 & 100 & sin delaminado \\
\hline & 70 & 5 & 80 & 160 & & 2.1 & 33.3 & 66.7 & \\
\hline & 80 & 160 & 102 & 138 & & 66.7 & 42.5 & 57.5 & \\
\hline \multirow[t]{5}{*}{ E08 } & 30 & 240 & 240 & 0 & & 100 & 100 & 0 & sin zona limpia \\
\hline & 50 & 240 & 240 & 0 & & 100 & 100 & 0 & sin zona limpia \\
\hline & 60 & 240 & 240 & 0 & 240 & 100 & 100 & 0 & sin zona limpia \\
\hline & 70 & 240 & 240 & 0 & & 100 & 100 & 0 & sin zona limpia \\
\hline & 80 & 240 & 240 & 0 & & 100 & 100 & 0 & sin zona limpia \\
\hline \multirow[t]{5}{*}{ E11 } & 30 & 240 & 205 & 35 & & 100 & 85.4 & 14.6 & \\
\hline & 50 & 68 & 240 & 0 & & 28.3 & 100 & 0 & sin zona limpia \\
\hline & 60 & 84.6 & 240 & 0 & 240 & 35.3 & 100 & 0 & sin zona limpia \\
\hline & 70 & 54.1 & 240 & 0 & & 23 & 100 & 0 & sin zona limpia \\
\hline & 80 & 98 & 180 & 60 & & 40.8 & 75 & 25 & \\
\hline \multirow[t]{5}{*}{ E12 } & 30 & 240 & 240 & 0 & & 100 & 100 & 0 & sin zona limpia \\
\hline & 50 & 170 & 100 & 140 & & 70.8 & 41.7 & 58.3 & \\
\hline & 60 & 39 & 205 & 35 & 240 & 16.3 & 85.4 & 14.6 & \\
\hline & 70 & 150 & 150 & 90 & & 62.5 & 62.5 & 37.5 & \\
\hline & 80 & 175 & 145 & 95 & & 72.9 & 60.4 & 39.6 & \\
\hline \multirow[t]{7}{*}{ E14 } & 1.1 & 177 & 90 & 150 & & 73.8 & 37.5 & 62.5 & \\
\hline & 5 & 76 & 230 & 10 & & 31.7 & 95.8 & 4.2 & \\
\hline & 10 & 173 & 240 & 0 & & 72.1 & 100 & 0 & sin zona limpia \\
\hline & 20 & 157.3 & 240 & 0 & 240 & 65.5 & 100 & 0 & sin zona limpia \\
\hline & 30 & 165 & 150 & 90 & & 68.8 & 62.5 & 37.5 & \\
\hline & 37.5 & 126 & 240 & 0 & & 52.5 & 100 & 0 & sin zona limpia \\
\hline & 40 & 185 & 240 & 0 & & 77.1 & 100 & 0 & sin zona limpia \\
\hline \multirow[t]{5}{*}{ E16 } & 30 & 145 & 181 & 59 & & 60.4 & 75.4 & 24.6 & \\
\hline & 50 & 120 & 150 & 90 & & 50 & 62.5 & 37.5 & \\
\hline & 60 & 140 & 240 & 0 & 240 & 58.3 & 100 & 0 & sin zona limpia \\
\hline & 70 & 130 & 130 & 110 & & 54.2 & 54.2 & 45.8 & \\
\hline & 80 & 135 & 240 & 0 & & 56.3 & 100 & 0 & sin zona limpia \\
\hline
\end{tabular}

Tras este análisis, respecto a la longitud de la pieza invadida por delaminado, se observa que el delaminado tipo I, en la mayoría de los 
experimentos y para los diferentes tiempos de mecanizado, tiene una presencia similar o inferior al del tipo II + III.

Respecto al delaminado tipo II + III, los experimentos con profundidad de pasada menor $\left(a_{\mathrm{p}}=0.5 \mathrm{~mm}\right)$ presentan menos delaminado y un mejor aspecto general de acabado en la pieza que los experimentos de profundidad de pasada mayor $\left(\mathrm{a}_{\mathrm{p}}=1.5 \mathrm{~mm}\right)$. Sólo uno de ellos tiene delaminado en toda la longitud mecanizada. Además, se observa que los experimentos realizados con $\mathrm{a}_{\mathrm{p}}=1.5$ $\mathrm{mm}$ tienen muy pocas zonas completamente libres de delaminado.

Aunque en este primer momento se han agrupado los tipos de delaminado II y III, porque ambos sobresalen del borde de la pieza, se van a realizar algunas consideraciones sobre el tipo III:

- No existe delaminado tipo III para orientaciones de tejido $\mathrm{Fo}=45^{\circ}-135^{\circ}$. Las hebras que sobresalen de la pieza sobre el borde mecanizado en la misma dirección del corte de la herramienta son las que generan el delaminado tipo III. Sin embargo, para estas orientaciones la disposición angular del tejido no permite ese tipo de delaminado.

- En los experimentos con orientación de fibra $\mathrm{Fo}=0^{\circ}-90^{\circ}$ y profundidad de pasada pequeña, se reduce mucho el efecto del delaminado que sobresale de la pieza, condicionado por el valor de la profundidad de pasada, por lo que tampoco en estos experimentos es fácil observar el delaminado tipo III.

- Este tipo de delaminado se puede apreciar sólo cuando la orientación de la trama está ligeramente inclinada respecto a la trayectoria de corte. Este tipo de delaminado puede aparecer porque la herramienta en su movimiento separa las fibras de la trama sin llegar a cortarlas.

- Es el de menor valor y el que menos se presenta en los experimentos realizados.

- Aparece de forma más evidente cuando la herramienta de corte presenta mayor desgaste.

- El delaminado tipo III nunca se produce solo, siempre aparece junto a los otros tipos de delaminado: tipos I y II.

Por todas estas razones, y debido a su pequeña influencia, el delaminado tipo III no será considerado en los análisis posteriores. 


\subsubsection{ANÁLISIS DEL DELAMINADO TIPO I Y TIPO II EN UN TEJIDO BIDIMENSIONAL}

La estructura del tejido elegido (Figura 4.25), afecta directamente al delaminado que se produce durante el mecanizado. Para cada tipo de tejido el efecto de delaminado será ligeramente diferente, y dependerá del entrecruzamiento de las mechas y el efecto soporte que ejercen entre ellas. El delaminado se produce únicamente en las capas más exteriores de la pieza por lo que interesa la disposición de las mechas en la capa superficial de material compuesto fabricada (Figura 4.26), donde la trama y la urdimbre del tejido pasan una por encima de la otra de forma alterna.

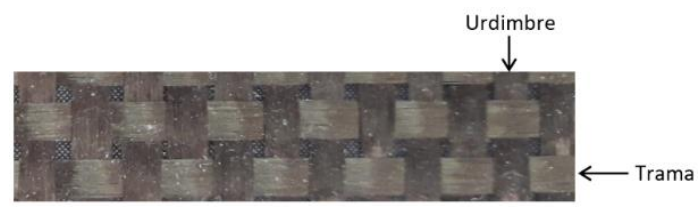

Figura 4.25. Tejido de basalto utilizado para la fabricación de las piezas

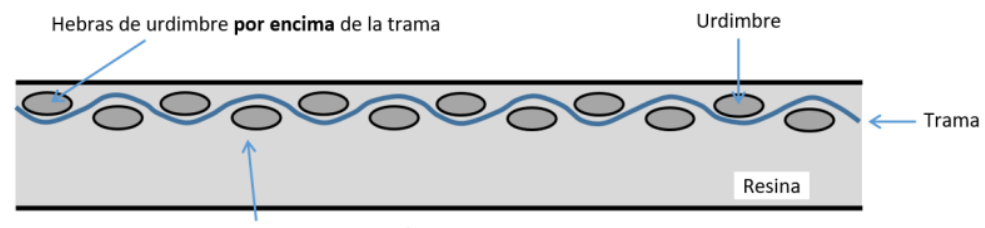

Hebras de urdimbre por debajo de la trama

Figura 4.26. Detalle de la capa superficial de tejido que sufre delaminado

En las Figuras 4.274 .28 y 4.29 se pueden observar algunos ejemplos del aspecto del delaminado de la capa superficial para diferentes orientaciones de fibra y diferentes tiempos de mecanizado.

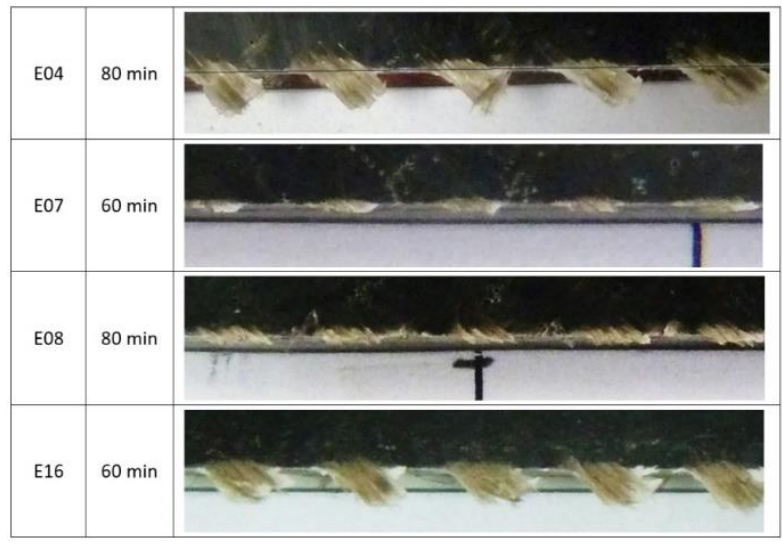

Figura 4.27. Aspecto delaminado para $\phi=45^{\circ}$. 


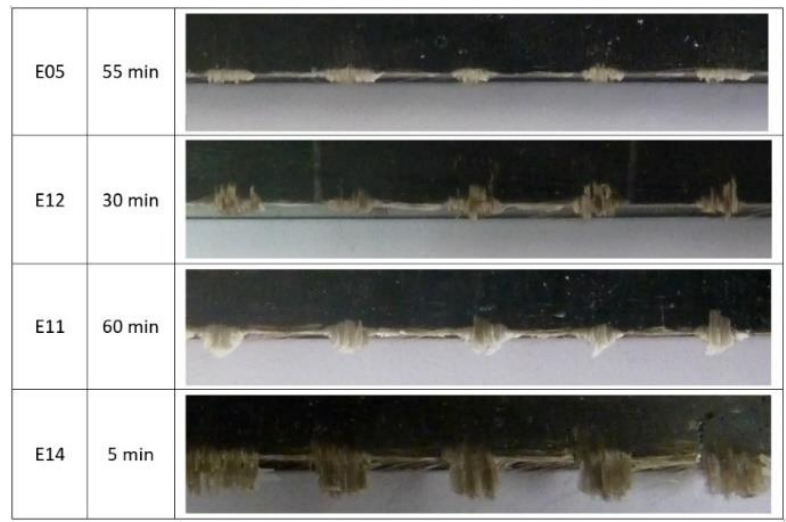

Figura 4.28. Aspecto delaminado para $\phi=90^{\circ}$.

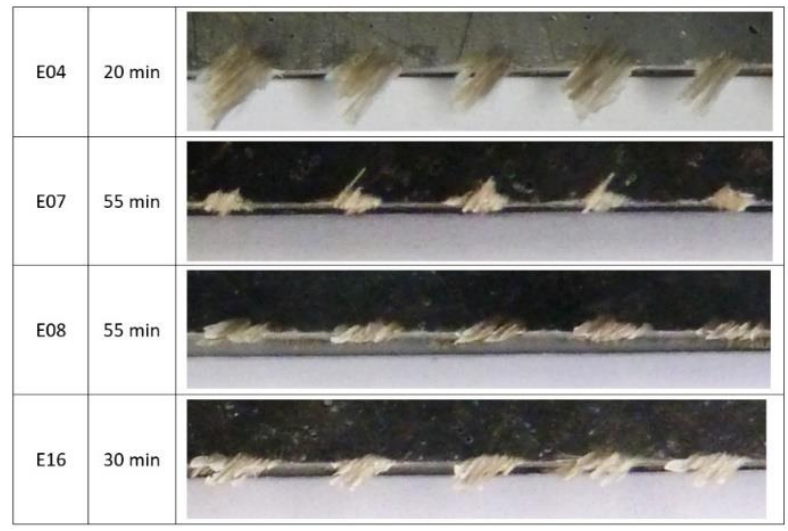

Figura 4.29. Aspecto delaminado para $\phi=135^{\circ}$.

Los experimentos con $\mathrm{Fo}=0^{\circ}-90^{\circ}$ y $\mathrm{Fo}=45^{\circ}-135^{\circ}$ presentan un aspecto diferente respecto al delaminado. Cuando hay delaminado en un tejido con $\mathrm{Fo}=45^{\circ}-135^{\circ}$, todas las mechas presentan delaminado porque sobre la línea que describe la trayectoria de corte de la herramienta, todos los hilos de urdimbre pasan por encima de la trama. Sin embargo, para en un tejido con orientación $\mathrm{Fo}=0^{\circ}-90^{\circ}$ es necesario estudiar diferentes casos, ya que el delaminado aparece sólo en la mecha de urdimbre que pasa por encima y en algunos casos en la que pasa por debajo de la trama, o en ambas. Además, en todas las orientaciones de tejido, se sigue apreciando el espacio libre entre mechas característico del entrecruzamiento del tejido.

En el caso del tejido con orientación $\mathrm{Fo}=0^{\circ}-90^{\circ}$, las mechas de urdimbre superficial (por encima de la trama) son más propensas al delaminado porque le falta el efecto soporte de la resina (Figura 4.30 (a)). 
Hay experimentos donde aparece además un delaminado ligero en las mechas de urdimbre intermedias donde los hilos pasan por debajo de la trama (Figura 4.30 (b)), y experimentos más desfavorables, donde aparece un delaminado abundante en ambas mechas, tanto en las que pasan por encima, como por debajo de la trama (Figura 4.30 (c)). Por tanto, para esta orientación de tejido, se han estudiado por separado las mechas de tejido por encima y/o por debajo de la trama [78].

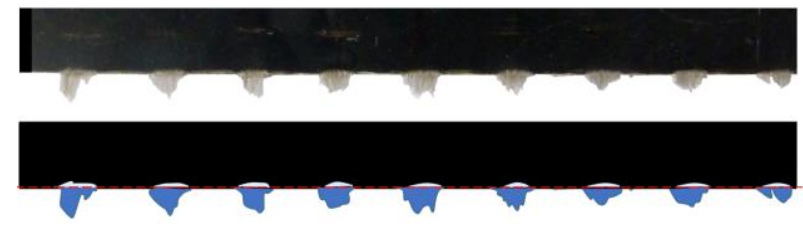

(a)

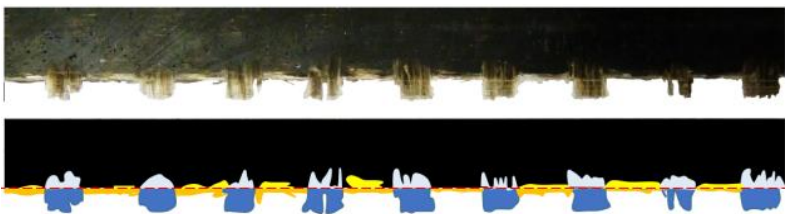

(b)
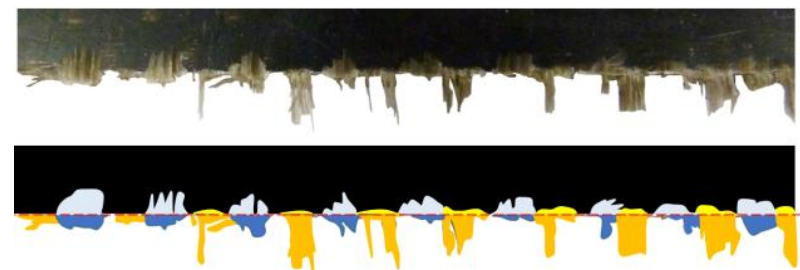

(c)

Delaminado en las mechas por debajo de la trama

Delaminado en las mechas por encima de la trama
Delaminado en las mechas por encima de la trama de tejido

Ligero delaminado en las mechas por debajo de la trama de tejido

Delaminado importante en las mechas por debajo de la trama de tejido

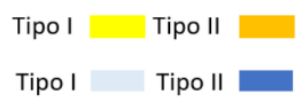

Figura 4.30. Superficie delaminada para Fo $0^{\circ}-90^{\circ}$.(a) delaminado en las mechas por encima de la trama $(b, c)$ delaminado en las mechas por encima y por debajo de la trama.

En la Figura 4.31 se puede observar una pieza con delaminado tipo I/II, pero con un corte limpio en las mechas que salen por debajo de la trama. Para poder analizar bien este efecto se han numerado las mechas como se indica en la figura, teniendo en cuenta que sólo tienen delaminado las mechas que salen por encima de la trama. 


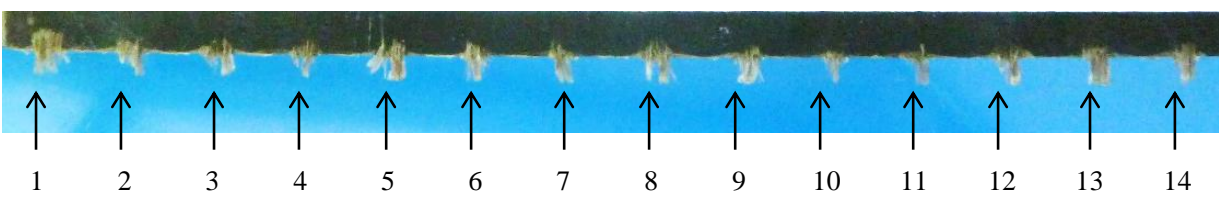

Figura 4.31. Numeración de mechas por encima de la trama de una pieza mecanizada

Por otro lado, existen muestras donde el delaminado aparece en casi todas las mechas de la urdimbre, independientemente de si van por encima o por debajo de la trama. La identificación de las mechas alternas que sobresalen por debajo de la trama se ha realizado numerando con "prima" a las mechas, según se indica en la Figura 4.32.

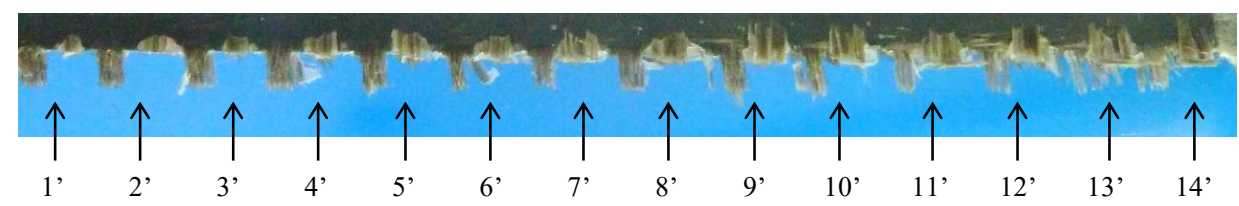

Figura 4.32. Numeración de mechas por debajo de la trama de una pieza mecanizada

En estos casos el delaminado se ha medido de la misma forma, pero considerando por separado las mechas de urdimbre que quedan por encima de la trama y las mechas que quedan por debajo, porque el efecto de delaminado es completamente distinto. En el ejemplo de la Figura 4.32, en las mechas que van por encima de la trama predomina el delaminado tipo II, mientras que en las mechas que van por debajo de la trama predomina el delaminado tipo I.

\subsection{ANÁLISIS CUANTITATIVO DEL DELAMINADO}

El análisis cuantitativo del delaminado, se ha llevado a cabo estudiando el delaminado en la zona más desfavorable de cada experimento denominada longitud evaluada (L) y que se ha fijado en $80 \mathrm{~mm}$ (Figura 4.33). En esta zona, se van a definir parámetros unidimensionales y de superficie que permitirán caracterizar el delaminado.

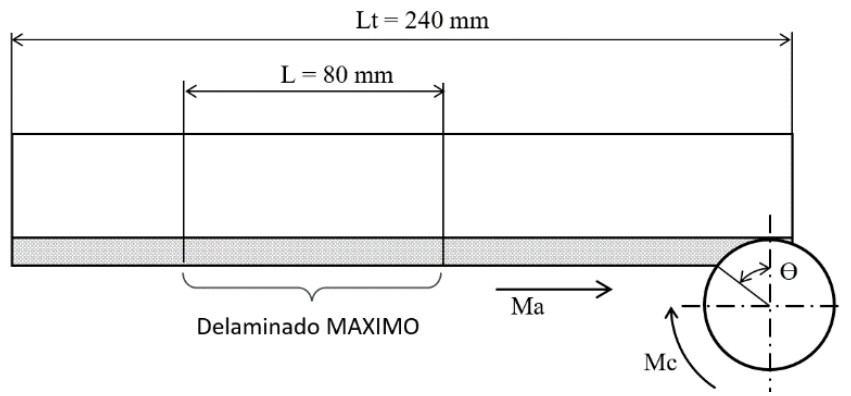

Figura 4.33. Longitud $(L)$ donde se ha evaluado el delaminado 
En los experimentos se ha buscado siempre una situación de delaminado uniforme, utilizando trayectorias que coinciden con las direcciones principales del tejido, que marcan puntos de inflexión en el comportamiento del material ante el mecanizado. Se trata de las orientaciones más desfavorables y, cualquier otra orientación de tejido intermedia que se produzca va a provocar un delaminado menor.

Los parámetros unidimensionales evalúan la longitud de las fibras que provocan el delaminado respecto al borde mecanizado, pero no definen correctamente la densidad del delaminado. Por lo tanto, es necesario definir parámetros de superficie para caracterizar mejor el delaminado.

Para comparar entre experimentos se define un factor de delaminación de área $(\mathrm{FSd})$ respecto al área delaminada máxima teórica posible para cada experimento.

En la zona más desfavorable de cada pieza se han medido los tramos de longitud de borde invadida (li), la altura máxima $\left(\mathrm{h}_{\left.\text {max }_{-}\right)}\right.$) y la superficie (Sdi) de cada una de las mechas delaminadas, tanto de las que van hacia dentro de la pieza (tipo I) como de las fibras que sobresalen (tipo II). En la Figura 4.34 se puede ver un ejemplo de cómo se realiza la medida.

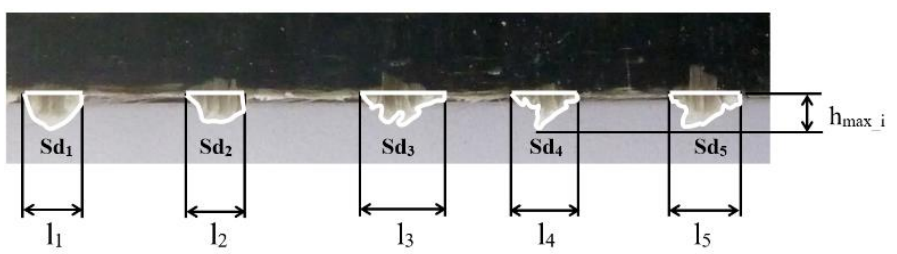

Figura 4.34. Ejemplo de medida de las características para delaminado tipo II.

A partir de las medidas individuales de cada mecha, se obtienen los valores que caracterizan cada experimento en la longitud evaluada (L). La longitud total que presenta delaminado (l) se obtiene como la suma de las longitudes invadidas por cada mecha. La superficie ( $\mathrm{Sd}$ ) se obtiene como la suma de las superficies individuales de cada mecha y la altura máxima $\left(\mathrm{h}_{\max }\right)$ como la altura de la mecha que más sobresale para cada tipo de delaminado (ecuaciones 4.4, 4.5 y 4.6$)$.

$$
\begin{array}{ll}
l=\sum l_{i} & \text { (ecuación 4.4) } \\
S d=\sum S d_{i} & \text { (ecuación 4.5) } \\
\mathrm{h}_{\max }=\max \left(\mathrm{h}_{\max }\right) & \text { (ecuación 4.6) }
\end{array}
$$


Al mismo tiempo se ha medido también el espacio libre entre mechas delaminadas ( $l_{\text {espacio libre }}$, que son los huecos sin delaminado que se producen dentro de la zona delaminada de la pieza. Este espacio depende del tipo de tejido y marca la forma en la que se produce el delaminado.

\subsubsection{PARÁMETROS UNIDIMENSIONALES}

En este punto se van a definir y medir los diferentes parámetros ( $\mathrm{Wa}, \mathrm{Wb})$ y factores (Fdel) unidimensionales para evaluar el delaminado en la tesis [78].

\subsubsection{Parámetro de delaminado medio (Wa)}

En primer lugar, se va a definir un parámetro que permita conocer la altura promedio del área delaminada para las fibras que sobresalen por encima de la trama, en el delaminado tipo II y para las fibras que van hacia adentro desde el borde recortado en el delaminado tipo I.

El parámetro de delaminado medio Wa (ecuación 4.7), se define como la altura de un rectángulo equivalente, con base la longitud evaluada (L), respecto a la superficie total delaminada ( $\mathrm{Sd}$ ). Esta longitud (L) considera tanto la longitud invadida por las fibras delaminadas (1), como el espacio libre entre mechas (l $l_{\text {espacio libre }}$ ), ecuación (4.8).

$$
\begin{array}{ll}
W_{a}=\frac{\sum S d_{i}}{\mathrm{~L}}=\frac{S d}{L} & \text { (ecuación 4.7) } \\
L=l+l_{\text {espacio libre }} & \text { (ecuación 4.8) }
\end{array}
$$

Este parámetro independiza la superficie delaminada de la longitud evaluada. En la Figura 4.35 se puede observar su aplicación al delaminado tipo II.

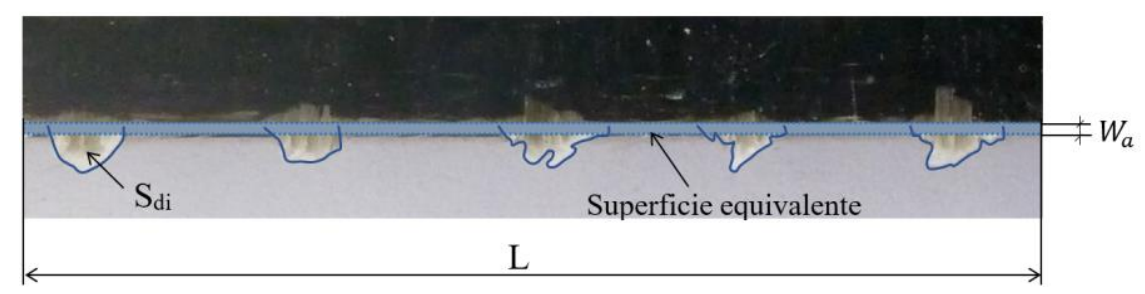

Figura 4.35. Parámetro de delaminado medio $\left(W_{a}\right)$ para tipo II.

En tejidos con orientación $\mathrm{Fo}=0^{\circ}-90^{\circ}$, este mismo parámetro (Wa') se ha definido para las mechas de tejido que sobresalen por debajo de la trama (ecuación 4.9).

$$
W_{a}^{\prime}=\frac{\sum S d_{i}^{\prime}}{\mathrm{L}}=\frac{S d^{\prime}}{\mathrm{L}}
$$

(ecuación 4.9) 
Este parámetro de delaminado medio (Wa) no ofrece una medida real de la altura delaminada, ya que al considerar también en el tramo evaluado el espacio libre entre mechas, el valor de la altura calculada es menor que la altura real delaminada.

\subsubsection{Parámetro de delaminado equivalente $(\mathrm{Wb})$}

Para conseguir un valor más real del delaminado, se define un segundo parámetro que permite conocer la altura promedio de las fibras únicamente respecto a la longitud (l) invadida por delaminado. Al eliminar el espacio libre que se produce entre las mechas delaminadas, el valor de altura promedio obtenido (Wb) se aproxima mejor a la altura real del delaminado. Este parámetro se calcula para las fibras que sobresalen en el delaminado tipo II y de las fibras que van hacia adentro desde el borde recortado en el delaminado tipo I en cada uno de los experimentos.

El parámetro de delaminado equivalente $\mathrm{Wb}$, se define como la altura de un rectángulo equivalente, con base en la longitud invadida (1), respecto a la superficie total delaminada (Sd), (ecuación 4.10). La aplicación de este parámetro al delaminado tipo II se puede observar en las Figuras 4.36 y 4.37.

$$
W_{b}=\frac{\sum S d_{i}}{\sum l_{i}}=\frac{S d}{l} \quad \text { (ecuación 4.10) }
$$

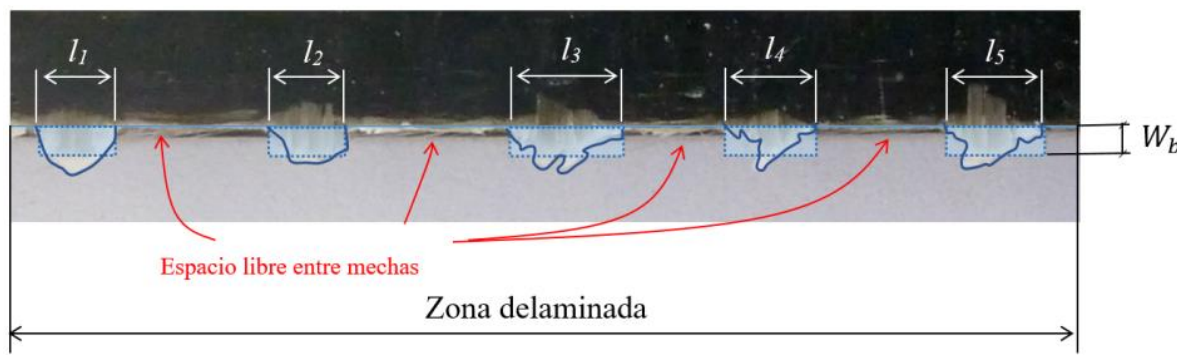

Figura 4.36. Medida de longitud y superficie delaminada tipo II.

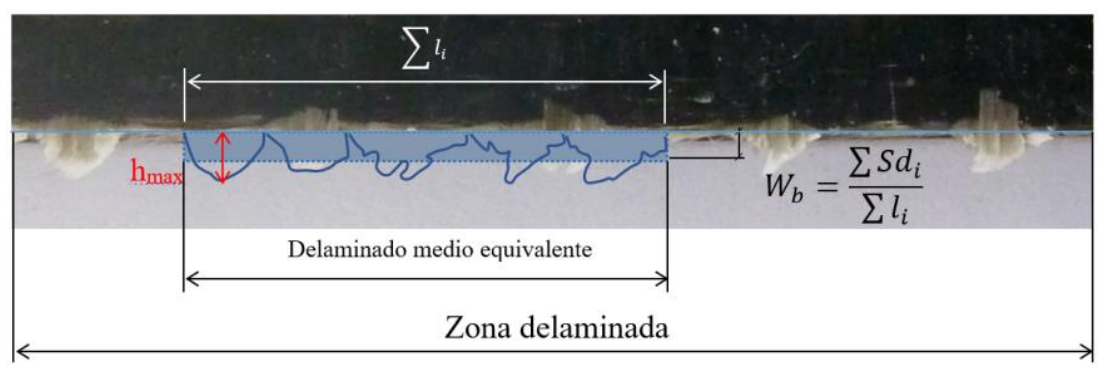

Figura 4.37. Parámetro de delaminado medio equivalente (Wb). 
En tejidos con orientación $\mathrm{Fo}=45^{\circ}-135$ el parámetro $\mathrm{Wb}$ representa bien el valor de delaminado medio para cada experimento. Todas las mechas del tejido sufren un delaminado equivalente debido a que todas ellas pasan por encima de la trama y tienen las mismas condiciones de disposición del tejido y de la resina respecto a la fuerza de corte.

Sin embargo, en tejidos con orientación $\mathrm{Fo}=0^{\circ}-90^{\circ}$ es necesario definir un parámetro distinto para las mechas que van por encima $(\mathrm{Wb})$ o por debajo (Wb') de la trama, ya que su comportamiento frente al delaminado es distinto por la disposición en el tejido. Para las mechas de tejido que sobresalen por debajo de la trama, en tejidos con orientación $\mathrm{Fo}=0^{\circ}-90^{\circ}$, se define $\mathrm{Wb}$, (ecuación 4.11) que considera sólo la superficie delaminada de las mechas que van por debajo de la trama (Sd') y su longitud invadida (l'). El valor de Wb' suele ser menor que el valor $\mathrm{Wb}$.

$$
W_{b}^{\prime}=\frac{\sum S d_{i}{ }^{\prime}}{\sum l_{i}{ }^{\prime}}=\frac{S d^{\prime}}{l^{\prime}}
$$

(ecuación 4.11)

El parámetro de delaminado equivalente $\mathrm{Wb}$ (ó Wb') es un parámetro que depende de la longitud delaminada. Si esta longitud es muy pequeña puede ser poco representativo. Además, los valores de $\mathrm{Wb}$ y Wb' son complementarios, pero no son aditivos ya que tienen un peso distinto a la hora de determinar el delaminado global.

\subsection{Valor máximo teórico $\left(W_{\max }\right)$ del parámetro de delaminado equivalente $(\mathrm{Wb})$.}

Como referencia para cuantificar el defecto de delaminado se han tomado los valores máximos teóricos $\left(\mathrm{W}_{\max }\right)$ que puede tomar el parámetro de delaminado $\mathrm{Wb}$. Estos valores máximos se establecen en función del tejido y de las condiciones de corte elegidas.

De esta forma para el delaminado tipo I, el valor máximo teórico esperado del parámetro $\mathrm{Wb}$ depende de la orientación de la fibra de tejido respecto a la trayectoria de corte. Para tejidos con $\mathrm{Fo}=0^{\circ}-90^{\circ}$ su valor coincide con el ancho de la mecha de tejido y en el caso de tejidos con $\mathrm{Fo}=45^{\circ}-135^{\circ}$, el delaminado máximo coincide con la diagonal de la mecha de tejido. Para el delaminado tipo II en cualquier orientación de tejido, el valor teórico máximo esperado coincide con la profundidad de pasada del mecanizado $\left(\mathrm{a}_{\mathrm{p}}\right)$. 


\subsubsection{Factor de delaminado (Fdel)}

Los distintos experimentos se han realizado con distintas profundidades de pasada $\left(a_{\mathrm{p}}\right)$, y esta variable afecta directamente al valor del delaminado equivalente $\mathrm{Wb}$. Para poder relacionar los experimentos realizados, se define un nuevo factor adimensional que valora el delaminado producido respecto a un máximo, y permite comparar los resultados de los experimentos realizados minimizando el efecto de la profundidad de pasada.

El factor de delaminado Fdel (ó Fdel') se define como la relación entre el delaminado equivalente $\mathrm{Wb}$ (ó $\mathrm{Wb}$ ') y el valor máximo teórico del delaminado $\left(\mathrm{W}_{\max }\right)$ tal y como se muestra en las ecuaciones 4.12 y 4.13.

$$
\begin{aligned}
\text { Fdel } & =\frac{W b}{W m a x} \\
\text { Fdel }^{\prime} & =\frac{W^{\prime}}{W m a x}
\end{aligned}
$$

\subsubsection{Resultados obtenidos}

En cada experimento, los datos de delaminado se han tomado para diferentes tiempos de corte, $\mathrm{Tc}=30,50,60,70$ y $80 \mathrm{~min}$. Los resultados obtenidos de los parámetros unidimensionales (Wa y $\mathrm{Wb}$ ) y el factor de delaminado (Fdel) tipo I y tipo II, se presentan en las Tablas 4.3 y 4.4. Estas tablas también muestran los valores máximos y mínimos de delaminado para los parámetros $\mathrm{Wa} \mathrm{y} \mathrm{Wb}$.

Tabla 4.3. Valor de los parámetros para el delaminado Tipo I.

\begin{tabular}{cccccccccc}
\hline \multirow{2}{*}{ Exp } & & Tc & Wa & Wa' & Wb & Wb' & Wmax & \multirow{2}{*}{ Fdel } & \multirow{2}{*}{ Fdel' } \\
\cline { 3 - 8 } & & $\mathrm{min}$ & $\mathrm{mm}$ & $\mathrm{mm}$ & $\mathrm{mm}$ & $\mathrm{mm}$ & $\mathrm{mm}$ & & \\
\hline \multirow{2}{*}{ E05 } & Vc 470 & 30 & 0.022 & 0.003 & 0.069 & 0.051 & 3 & 0.0231 & 0.0171 \\
& Fz 0.1 & 50 & 0.031 & 0.008 & 0.080 & 0.084 & 3 & 0.0265 & 0.0281 \\
& ap 0.5 & 60 & 0.033 & 0.013 & 0.098 & 0.098 & 3 & 0.0326 & 0.0327 \\
& Fo 90 & 70 & 0.025 & 0.023 & 0.064 & 0.058 & 3 & 0.0215 & 0.0192 \\
& & 80 & 0.034 & 0.012 & 0.105 & 0.051 & 3 & 0.0348 & 0.0169 \\
\hline \multirow{2}{*}{ E12 } & Vc 300 & 30 & 0.065 & 0.005 & 0.174 & 0.049 & 3 & 0.0579 & 0.0164 \\
& Fz 0.4 & 50 & 0.048 & 0 & 0.118 & 0 & 3 & 0.0394 & 0 \\
& ap 0.5 & 60 & 0.053 & 0 & 0.131 & 0 & 3 & 0.0437 & 0 \\
& Fo 90 & 70 & 0.062 & 0.004 & 0.160 & 0.091 & 3 & 0.0534 & 0.0303 \\
& & 80 & 0.058 & 0.007 & 0.145 & 0.029 & 3 & 0.0485 & 0.0097 \\
\hline
\end{tabular}


CAPÍTULO 4. ANÁLISIS DEL DELAMINADO

\begin{tabular}{|c|c|c|c|c|c|c|c|c|c|}
\hline \multirow{2}{*}{ Exp } & & $\mathrm{Tc}$ & Wa & Wa' & $\mathrm{Wb}$ & $\mathrm{Wb}$ & Wmax & \multirow{2}{*}{ Fdel } & \multirow{2}{*}{ Fdel' } \\
\hline & & $\min$ & $\mathrm{mm}$ & $\mathrm{mm}$ & $\mathrm{mm}$ & $\mathrm{mm}$ & $\mathrm{mm}$ & & \\
\hline \multirow[t]{5}{*}{ E11 } & Vc 300 & 30 & 0.081 & 0 & 0.178 & 0 & 3 & 0.0592 & 0 \\
\hline & $\mathrm{Fz} 0.1$ & 50 & 0.093 & 0 & 0.215 & 0 & 3 & 0.0718 & 0 \\
\hline & ap 1.5 & 60 & 0.073 & 0.026 & 0.162 & 0.079 & 3 & 0.0539 & 0.0264 \\
\hline & Fo $90^{\circ}$ & 70 & 0.049 & 0.025 & 0.131 & 0.080 & 3 & 0.0438 & 0.0267 \\
\hline & & 80 & 0.063 & 0.054 & 0.133 & 0.102 & 3 & 0.0445 & 0.0340 \\
\hline \multirow[t]{7}{*}{ E14 } & Vc 470 & 1.1 & 0.183 & 0.011 & 0.392 & 0.071 & 3 & 0.1308 & 0.0235 \\
\hline & $\mathrm{Fz} 0.4$ & 5 & 0.147 & 0.124 & 0.309 & 0.272 & 3 & 0.1029 & 0.0905 \\
\hline & ap 1.5 & 10 & 0.222 & 0.154 & 0.498 & 0.278 & 3 & 0.1661 & 0.0928 \\
\hline & Fo $90^{\circ}$ & 20 & 0.351 & 0.094 & 0.852 & 0.161 & 3 & 0.2839 & 0.0535 \\
\hline & & 30 & 0.331 & 0.054 & 0.856 & 0.098 & 3 & 0.2852 & 0.0326 \\
\hline & & 37.5 & 0.161 & 0.012 & 0.430 & 0.070 & 3 & 0.1433 & 0.0232 \\
\hline & & 40 & 0.077 & 0.318 & 0.171 & 0.656 & 3 & 0.0571 & 0.2187 \\
\hline \multirow[t]{5}{*}{ E07 } & Vc 300 & 30 & 0.007 & & 0.139 & & 4.24 & 0.0327 & \\
\hline & $\mathrm{Fz} 0.1$ & 50 & 0.005 & & 0.086 & & 4.24 & 0.0202 & \\
\hline & ap 0.5 & 60 & 0.017 & & 0.085 & & 4.24 & 0.0201 & \\
\hline & Fo $45^{\circ}$ & 70 & 0 & & 0 & & 4.24 & 0 & \\
\hline & & 80 & 0.025 & & 0.126 & & 4.24 & 0.0296 & \\
\hline \multirow[t]{5}{*}{ E08 } & Vc 470 & 30 & 0.126 & & 0.266 & & 4.24 & 0.0626 & \\
\hline & $\mathrm{Fz} 0.4$ & 50 & 0.121 & & 0.206 & & 4.24 & 0.0486 & \\
\hline & ap 0.5 & 60 & 0.098 & & 0.185 & & 4.24 & 0.0436 & \\
\hline & Fo $45^{\circ}$ & 70 & 0.122 & & 0.305 & & 4.24 & 0.0719 & \\
\hline & & 80 & 0.032 & & 0.081 & & 4.24 & 0.0190 & \\
\hline \multirow[t]{5}{*}{ E04 } & Vc 300 & 30 & 0.164 & & 0.308 & & 4.24 & 0.0726 & \\
\hline & $\mathrm{Fz} 0.4$ & 50 & 0.105 & & 0.225 & & 4.24 & 0.0531 & \\
\hline & ap 1.5 & 60 & 0.137 & & 0.238 & & 4.24 & 0.0562 & \\
\hline & Fo $45^{\circ}$ & 70 & 0.104 & & 0.183 & & 4.24 & 0.0430 & \\
\hline & & 80 & 0.153 & & 0.252 & & 4.24 & 0.0593 & \\
\hline \multirow[t]{5}{*}{ E16 } & Vc 470 & 30 & 0.039 & & 0.098 & & 4.24 & 0.0231 & \\
\hline & Fz 0.1 & 50 & 0.121 & & 0.249 & & 4.24 & 0.0587 & \\
\hline & ap 1.5 & 60 & 0.063 & & 0.098 & & 4.24 & 0.0231 & \\
\hline & Fo $45^{\circ}$ & 70 & 0.034 & & 0.093 & & 4.24 & 0.0219 & \\
\hline & & 80 & 0.086 & & 0.180 & & 4.24 & 0.0424 & \\
\hline
\end{tabular}


Tabla 4.4. Valor de los parámetros para el delaminado Tipo II.

\begin{tabular}{|c|c|c|c|c|c|c|c|c|c|}
\hline \multirow{2}{*}{ Exp } & & $\mathrm{Tc}$ & Wa & Wa' & $\mathrm{Wb}$ & $\mathrm{Wb}$ ' & Wmax & \multirow{2}{*}{ Fdel } & \multirow{2}{*}{ Fdel' } \\
\hline & & $\min$ & $\mathrm{mm}$ & $\mathrm{mm}$ & $\mathrm{mm}$ & $\mathrm{mm}$ & $\mathrm{mm}$ & & \\
\hline \multirow[t]{5}{*}{ E05 } & Vc 470 & 30 & 0.065 & 0.015 & 0.201 & 0.170 & 0.5 & 0.4015 & 0.3404 \\
\hline & $\mathrm{Fz} 0.1$ & 50 & 0.066 & 0.007 & 0.170 & 0.098 & 0.5 & 0.3402 & 0.1951 \\
\hline & ap 0.5 & 60 & 0.057 & 0.062 & 0.169 & 0.131 & 0.5 & 0.3386 & 0.2354 \\
\hline & Fo $90^{\circ}$ & 70 & 0.105 & 0.091 & 0.276 & 0.178 & 0.5 & 0.5522 & 0.3558 \\
\hline & & 80 & 0.052 & 0.062 & 0.157 & 0.164 & 0.5 & 0.3137 & 0.2730 \\
\hline \multirow[t]{5}{*}{ E12 } & Vc 300 & 30 & 0.154 & 0.075 & 0.369 & 0.235 & 0.5 & 0.7371 & 0.4690 \\
\hline & $\mathrm{Fz} 0.4$ & 50 & 0.067 & 0.022 & 0.165 & 0.136 & 0.5 & 0.3306 & 0.2722 \\
\hline & ap 0.5 & 60 & 0.067 & 0.009 & 0.167 & 0.127 & 0.5 & 0.3336 & 0.2549 \\
\hline & Fo $90^{\circ}$ & 70 & 0.067 & 0.010 & 0.173 & 0.175 & 0.5 & 0.3467 & 0.3498 \\
\hline & & 80 & 0.113 & 0.117 & 0.284 & 0.249 & 0.5 & 0.5688 & 0.4783 \\
\hline \multirow[t]{5}{*}{ E11 } & Vc 300 & 30 & 0.109 & 0.025 & 0.239 & 0.093 & 1.5 & 0.1591 & 0.0618 \\
\hline & $\mathrm{Fz} 0.1$ & 50 & 0.292 & 0.016 & 0.678 & 0.080 & 1.5 & 0.4520 & 0.0533 \\
\hline & ap 1.5 & 60 & 0.530 & 0.102 & 1.176 & 0.215 & 1.5 & 0.7839 & 0.1432 \\
\hline & Fo $90^{\circ}$ & 70 & 0.275 & 0.103 & 0.743 & 0.196 & 1.5 & 0.4952 & 0.1306 \\
\hline & & 80 & 0.276 & 0.070 & 0.581 & 0.133 & 1.5 & 0.3875 & 0.0886 \\
\hline \multirow[t]{7}{*}{ E14 } & Vc 470 & 1.1 & 0.293 & 0.099 & 0.627 & 0.266 & 1.5 & 0.4180 & 0.1772 \\
\hline & $\mathrm{Fz} 0.4$ & 5 & 0.643 & 0.179 & 1.352 & 0.341 & 1.5 & 0.9012 & 0.2270 \\
\hline & ap 1.5 & 10 & 0.538 & 0.368 & 1.207 & 0.663 & 1.5 & 0.8045 & 0.4422 \\
\hline & Fo $90^{\circ}$ & 20 & 0.464 & 0.201 & 1.125 & 0.342 & 1.5 & 0.7502 & 0.2282 \\
\hline & & 31 & 0.515 & 0.222 & 1.333 & 0.401 & 1.5 & 0.8884 & 0.2674 \\
\hline & & 37.5 & 0.484 & 0.166 & 1.293 & 0.362 & 1.5 & 0.8623 & 0.2410 \\
\hline & & 40 & 0.841 & 0.308 & 1.875 & 0.634 & 1.5 & 1.2502 & 0.4226 \\
\hline \multirow[t]{5}{*}{ E07 } & Vc 300 & 30 & 0.028 & & 0.208 & & 0.5 & 0.4160 & \\
\hline & $\mathrm{Fz} 0.1$ & 50 & 0.021 & & 0.120 & & 0.5 & 0.2400 & \\
\hline & ap 0.5 & 60 & 0.009 & & 0.056 & & 0.5 & 0.1112 & \\
\hline & Fo $45^{\circ}$ & 70 & 0.030 & & 0.182 & & 0.5 & 0.3646 & \\
\hline & & 80 & 0.039 & & 0.149 & & 0.5 & 0.2971 & \\
\hline \multirow[t]{5}{*}{ E08 } & Vc 470 & 30 & 0.189 & & 0.397 & & 0.5 & 0.7932 & \\
\hline & Fz 0.4 & 50 & 0.165 & & 0.283 & & 0.5 & 0.5654 & \\
\hline & ap 0.5 & 60 & 0.136 & & 0.256 & & 0.5 & 0.5115 & \\
\hline & Fo $45^{\circ}$ & 70 & 0.168 & & 0.417 & & 0.5 & 0.8349 & \\
\hline & & 80 & 0.128 & & 0.213 & & 0.5 & 0.4250 & \\
\hline
\end{tabular}




\begin{tabular}{|c|c|c|c|c|c|c|c|c|c|}
\hline \multirow{2}{*}{ Exp } & & $\mathrm{Tc}$ & Wa & Wa' & $\mathrm{Wb}$ & $\mathrm{Wb}^{\prime}$ & Wmax & \multirow{2}{*}{ Fdel } & \multirow{2}{*}{ Fdel' } \\
\hline & & $\min$ & $\mathrm{mm}$ & $\mathrm{mm}$ & $\mathrm{mm}$ & $\mathrm{mm}$ & $\mathrm{mm}$ & & \\
\hline \multirow[t]{5}{*}{ E04 } & Vc 300 & 30 & 0.372 & & 0.745 & & 1.5 & 0.4967 & \\
\hline & $\mathrm{Fz} 0.4$ & 50 & 0.498 & & 1.072 & & 1.5 & 0.7148 & \\
\hline & ap 1.5 & 60 & 0.596 & & 1.038 & & 1.5 & 0.6921 & \\
\hline & Fo $45^{\circ}$ & 70 & 0.293 & & 0.512 & & 1.5 & 0.3412 & \\
\hline & & 80 & 0.597 & & 0.983 & & 1.5 & 0.6551 & \\
\hline \multirow[t]{5}{*}{ E16 } & Vc 470 & 30 & 0.387 & & 0.848 & & 1.5 & 0.5652 & \\
\hline & $\mathrm{Fz} 0.1$ & 50 & 0.248 & & 0.513 & & 1.5 & 0.3420 & \\
\hline & ap 1.5 & 60 & 0.641 & & 1.004 & & 1.5 & 0.6693 & \\
\hline & Fo $45^{\circ}$ & 70 & 0.230 & & 0.683 & & 1.5 & 0.4552 & \\
\hline & & 80 & 0.245 & & 0.445 & & 1.5 & 0.2969 & \\
\hline
\end{tabular}

A medida que aumenta el tiempo de corte (Tc), aumenta el desgaste de la herramienta $(\mathrm{Vb})$ y el delaminado debería ser mayor, aumentando los valores de los parámetros y factores. Sin embargo, los valores de delaminado presentan una gran variación entre los diferentes tiempos de corte, sin mostrar una tendencia creciente o decreciente a medida que aumenta el desgaste de la herramienta, como si se tratase de un fenómeno aleatorio. Además, los resultados en todos los casos dan valores más altos para el delaminado tipo II. Tras analizar los parámetros calculados, se confirma que no existe correlación clara entre el delaminado y el tiempo de corte o su influencia es muy pequeña, dentro del intervalo estudiado (hasta $\mathrm{Tc}=80 \mathrm{~min}$ ).

Esto se debe a que hay otro parámetro en el proceso (Xd), que tiene más influencia sobre la variación del delaminado que los relacionados con el mecanizado. La distancia desde el borde recortado hasta el siguiente cruce entre mechas de tejido es diferente en cada experimento y tiempo de corte. Esta distancia depende de la orientación de la fibra y del ancho de la mecha de tejido, así como del ángulo de inclinación de la tela con la trayectoria de corte. Tal y como se ha expuesto, Xd puede tomar un valor constante, puede seguir un patrón uniforme o puede ser totalmente aleatorio, Figura 4.38. Por este motivo, los valores mínimo y máximo de los parámetros y factores de delaminado analizados no se presentan para los mismos tiempos de corte, concluyendo que en los experimentos realizados prácticamente no existe dependencia entre el delaminado y el tiempo de corte. 


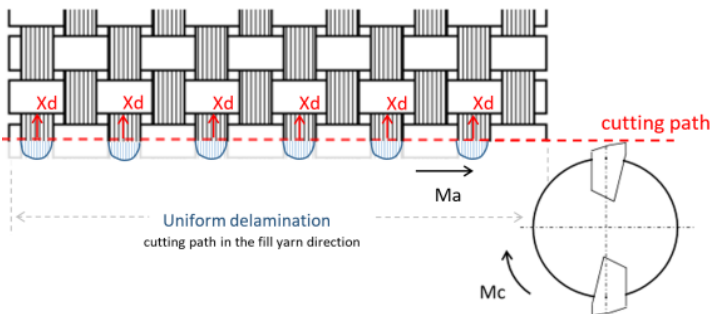

(a) Xd constante

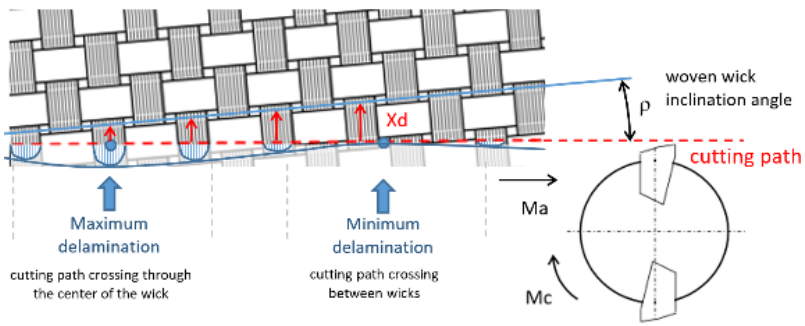

(b) Xd patrón de variación uniforme

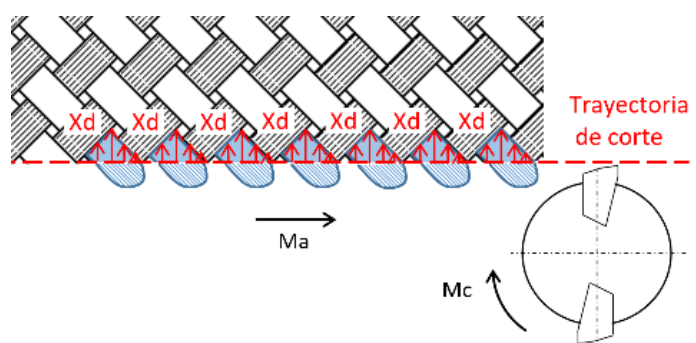

(c) Xd patrón de variación uniforme

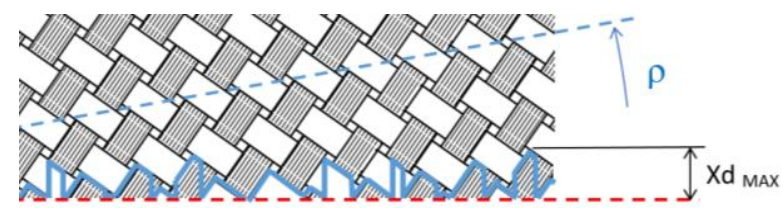

(d) Xd aleatorio delaminado

uniforme

$\mathrm{Fo}=0^{\circ}-90^{\circ}$

efecto onda

$\mathrm{Fo}=\left(0^{\circ}-90^{\circ}\right)+\rho$

tejido ligeramente inclinado

delaminado uniforme $\mathrm{Fo}=45^{\circ}-135^{\circ}$

delaminado aleatorio $\mathrm{Fo}=\left(45^{\circ}-135^{\circ}\right)+\rho$ tejido ligeramente inclinado

Figura 4.38. Variación de Xd.

La Tabla 4.5 compara la media de los parámetros de delaminado para el tipo I y tipo II, observando que todos los valores para el tipo II son mayores que para el tipo I. Por este motivo, se puede concluir que el delaminado tipo II es más importante que el tipo I, observando que el factor de delaminado (Fdel) para el tipo II es once veces mayor que el del tipo I. 
Tabla 4.5. Comparación de parámetros Tipo I y Tipo II.

\begin{tabular}{|c|c|c|c|}
\hline & $\overline{\mathrm{W}_{\mathrm{a}}}(\mathrm{mm})$ & $\overline{\mathrm{W}_{\mathrm{b}}}(\mathrm{mm})$ & $\overline{\mathrm{F}_{\mathrm{del}}}$ \\
\hline Tipo I & 0.067 & 0.153 & 0.042 \\
\hline \multirow[t]{2}{*}{ Tipo II } & 0.209 & 0.448 & 0.467 \\
\hline & $\frac{\overline{\mathrm{W}_{\mathrm{a}}} \text { tipo II }}{\overline{\mathrm{W}_{\mathrm{a}}} \text { tipo I }}=3.12$ & $\frac{\overline{\mathrm{W}_{\mathrm{b}}} \text { tipo II }}{\overline{\mathrm{W}_{\mathrm{b}}} \text { tipo I }}=2.93$ & $\frac{\overline{\mathrm{F}_{\text {del }}} \text { tipo II }}{\overline{\mathrm{F}_{\mathrm{del}}} \text { tipo I }}$ \\
\hline
\end{tabular}

En los experimentos con $\mathrm{Fo}=0^{\circ}-90^{\circ}$, el parámetro Wa normalmente es mayor que Wa'. En las tablas 4.3 y 4.4 se puede observar que hay muy pocos casos donde tengan valores similares (valores coloreados en verde). Por este motivo, se concluye que el delaminado en las hebras por debajo de la trama en la mayoría de los casos se podría despreciar.

Por otro lado, es necesario comprobar el valor de la longitud de borde invadida, cuando el delaminado equivalente $\mathrm{Wb}$ tiene valores similares a $\mathrm{Wb}$ '. En este caso, el valor del delaminado producido es similar para las mechas que pasan por encima de la trama y las que pasan por debajo de la trama. Cuando estas longitudes sean representativas, los factores $\mathrm{Wb}$ y $\mathrm{Wb}$ ' deben considerarse juntos para evaluar el delaminado producido. En estos casos el borde mecanizado de las piezas aparece completamente delaminado sin apenas espacio libre entre mechas.

Los experimentos donde los valores "prima" tienen un peso importante frente a los "no prima", se muestran en la Tabla 4.6. Este efecto se tendrá en cuenta en el cálculo de los factores de superficie que se mostrará más adelante.

Tabla 4.6. Experimentos donde considerar los valores "prima"

\begin{tabular}{|c|c|c|c|c|c|c|c|c|}
\hline \multirow[b]{2}{*}{ Exp } & & \multirow[b]{2}{*}{$\begin{array}{l}\text { Tc } \\
\text { min }\end{array}$} & \multicolumn{3}{|c|}{ Tipo I } & \multicolumn{3}{|c|}{ Tipo II } \\
\hline & & & $\mathrm{Wb}$ & $\begin{array}{l}\mathrm{Wb}^{\prime} \\
\mathrm{mm}\end{array}$ & $\begin{array}{c}\mathrm{l}^{\prime} \\
\mathrm{mm}\end{array}$ & $\mathrm{Wb}$ & $\begin{array}{l}\mathrm{Wb} \\
\mathrm{mm}\end{array}$ & $\begin{array}{c}\text { l' } \\
\mathrm{mm}\end{array}$ \\
\hline \multirow[t]{5}{*}{ E05 } & Vc 470 & 30 & 0.069 & 0.051 & 4.2 & 0.201 & 0.170 & 6.9 \\
\hline & Fz 0.1 & 50 & 0.080 & 0.084 & 7.5 & 0.170 & 0.098 & 5.7 \\
\hline & ap 0.5 & 60 & 0.098 & 0.098 & 10.3 & 0.169 & 0.131 & 38.2 \\
\hline & Fo $90^{\circ}$ & 70 & 0.065 & 0.058 & 31.9 & 0.276 & 0.178 & 40.9 \\
\hline & & 80 & 0.105 & 0.051 & 18.4 & 0.157 & 0.164 & 30.0 \\
\hline
\end{tabular}




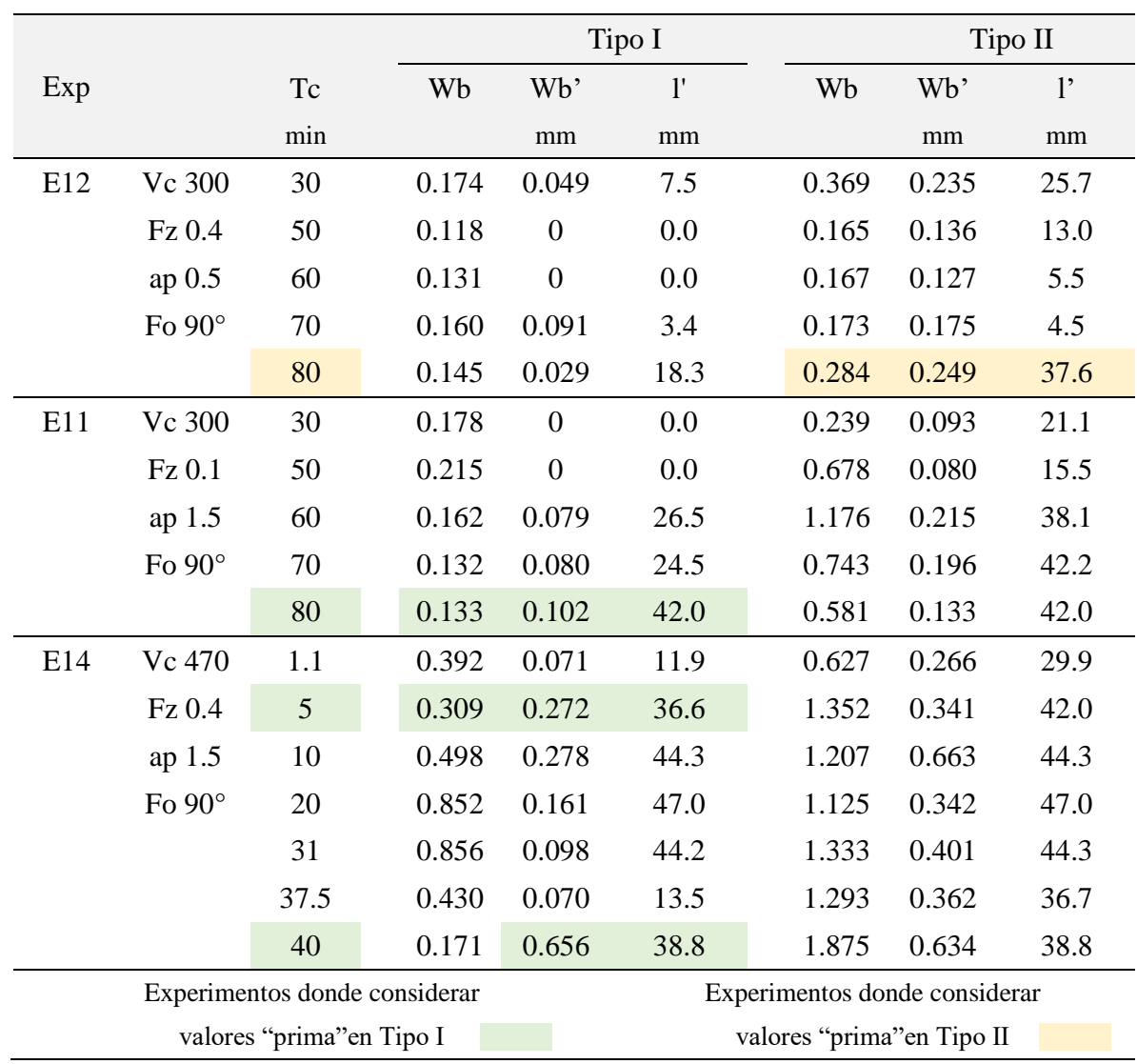

\subsubsection{Rangos de delaminado}

Los parámetros y factores de delaminado unidimensionales calculados para cada experimento, presentan una gran variación en los diferentes tiempos de corte, sin una tendencia clara creciente o decreciente. Esto es debido a qué con las mismas condiciones de corte, se pueden obtener resultados de delaminado distintos, por la variación del parámetro Xd.

Para su análisis, se ha definido un rango de variación, desde el valor mínimo hasta el máximo observado y que representa el delaminado obtenido para cada uno de los experimentos (Figuras 4.39 y 4.40) [78].

Al observar las gráficas de rangos de delaminado para el tipo II, tanto para Wa como para $\mathrm{Wb}$, se distingue con claridad que los experimentos que tienen mayor profundidad de pasada $\left(a_{\mathrm{p}}=1.5\right)$, presentan valores y rangos de delaminado mayores (E11, E14, E04 y E16). Esto no sucede para el delaminado tipo I. 
En los experimentos con profundidad de pasada pequeña $\left(a_{p}=0.5\right)$ los valores de delaminado tipo I y II tienen valores del mismo orden de magnitud, pero siendo ligeramente superior el tipo II frente al tipo I en casi todos los casos (E05, E12, E07 y E08).

El experimento $\mathrm{E} 14\left(\mathrm{Vc}=470, \mathrm{Fz}=0.4, \mathrm{a}_{\mathrm{p}}=1.5, \mathrm{Fo}=90^{\circ}\right)$ destaca por tener con diferencia los peores resultados de delaminado, debido a que las condiciones de este experimento son las más desfavorables.
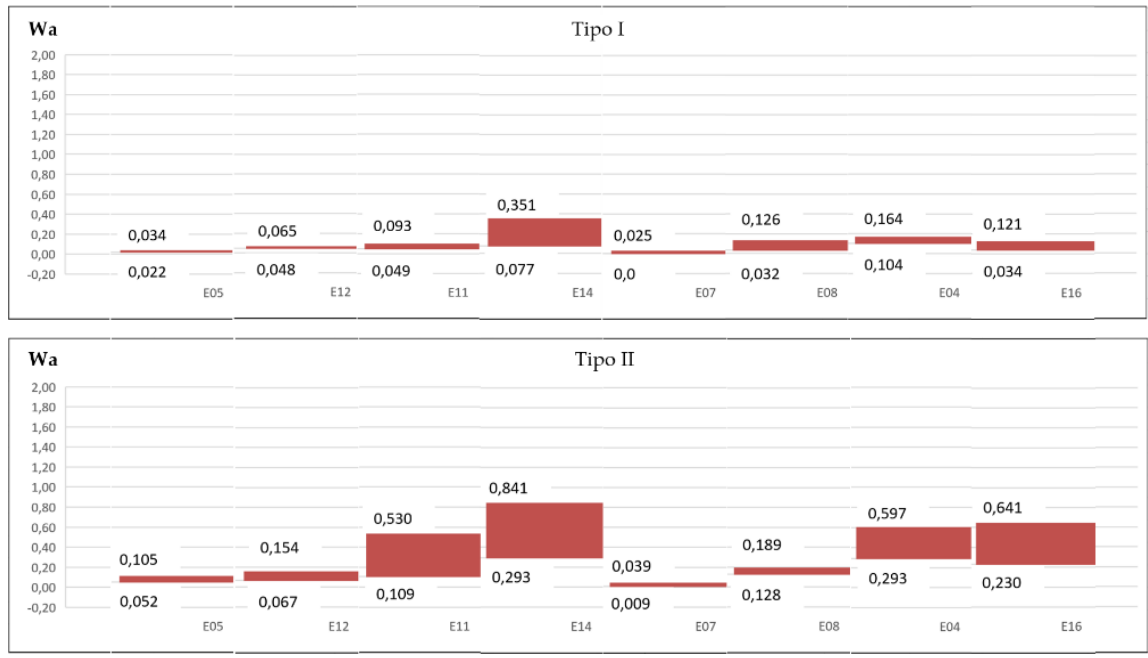

Figura 4.39. Rango del parámetro Wa
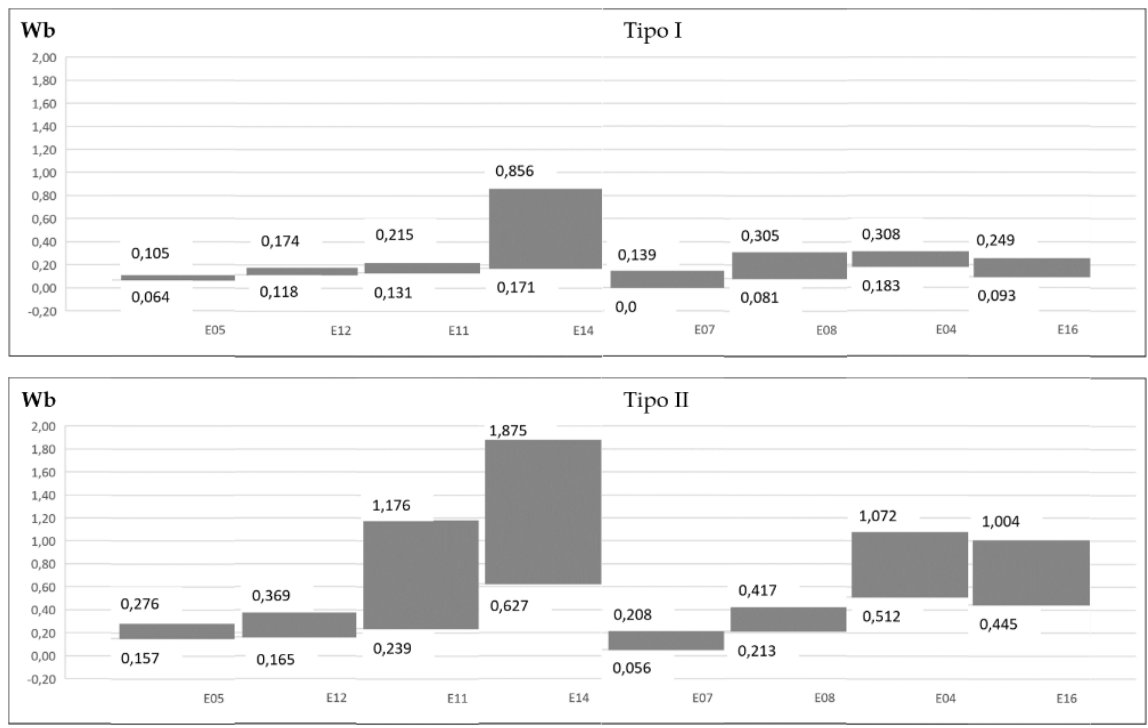

Figura 4.40. Rango del parámetro Wb 

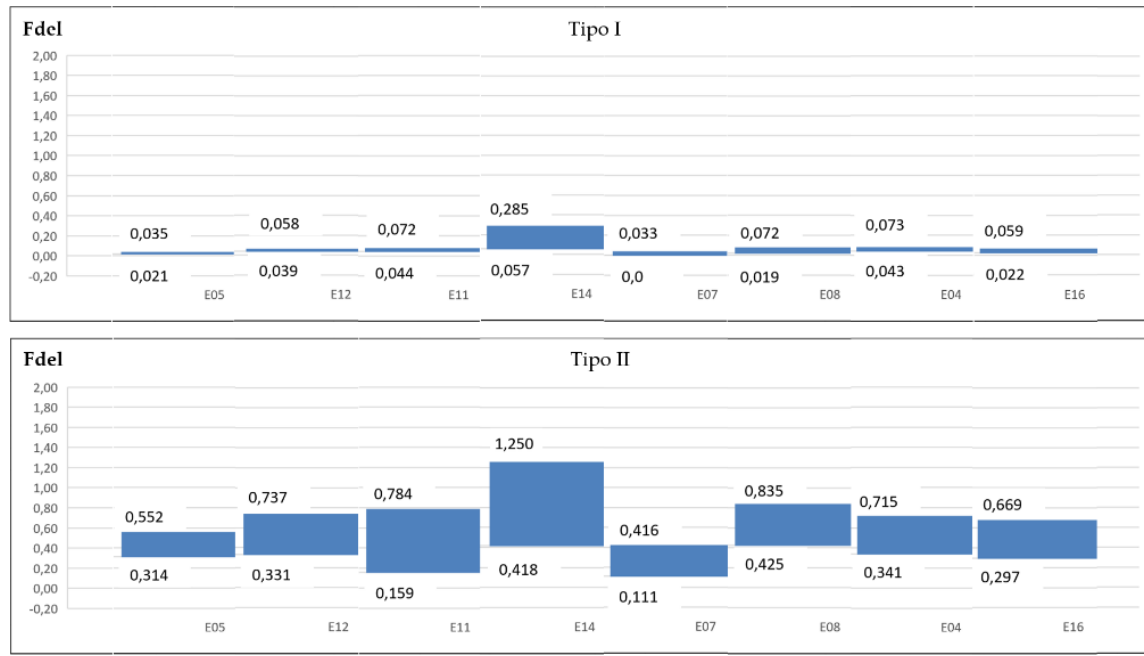

Figura 4.41. Factor de delaminado, Fdel

Al representar el rango para el factor de delaminado Fdel, se puede observar mejor la diferencia entre el delaminado tipo I y el de tipo II. El tipo II tiene mucha más variación (Figura 4.41).

Respecto a los valores del factor de delaminado, en el tipo I la variación de parámetros del diseño de experimentos no se observa en el gráfico de rango. En todos los casos, el valor máximo representa menos de un $8 \%$ del máximo teórico. Sin embargo, el delaminado tipo II tiene mayor rango de variación en todos los experimentos.

Los experimentos con valores más bajos y menor rango de variación, y por lo tanto con mejores condiciones desde el punto de vista del delaminado tipo II son el E05 ( $\left.\mathrm{Vc}=470, \mathrm{Fz}=0.1, \mathrm{a}_{\mathrm{p}}=0.5, \mathrm{Fo}=90^{\circ}\right)$ y el E07 $(\mathrm{Vc}=300, \mathrm{Fz}=0.1$, $\mathrm{a}_{\mathrm{p}}=0.5, \mathrm{Fo}=45^{\circ}$ ).

\subsubsection{Análisis de la profundidad de pasada}

La profundidad de pasada es un parámetro que la mayoría de los autores no han considerado. Sin embargo, el valor máximo del delaminado tipo II está limitado directamente por la profundidad de pasada $\left(a_{p}\right)$. Tanto la dimensión de las mechas que sobresalen, como la superficie que ocupan, son consecuencia del valor de este parámetro. Cuando aparece delaminado tipo II en un experimento, este es mucho mayor, conforme mayor es la profundidad 
de pasada, por lo que no es posible comparar los valores de delaminado tipo II de piezas mecanizadas con profundidades de pasada diferentes.

En esta tesis se ha trabajado con una profundidad de pasada adecuada al tamaño de la mecha $(3 \mathrm{~mm})$. Esta profundidad varía entre 0.5 y $1.5 \mathrm{~mm}$ para permitir observar el efecto de la incisión de la herramienta dentro de la mecha.

En el delaminado tipo II se observa que el valor máximo en algún caso es mayor que $a_{p}$ (Figuras 4.42 y 4.43) [77]. Esto se debe a la forma original del tejido bidimensional que se ha utilizado para realizar los experimentos. Este tejido es plano en dos dimensiones, con hebras entrecruzadas una a una, donde la longitud total de la hebra se presenta de forma ondulada. Al mecanizar el material se deshace el zig-zag que entrecruza la trama y la urdimbre. La mecha que no ha sido cortada por la herramienta se estira y puede ser de mayor tamaño que la profundidad de pasada utilizada (ecuación 4.14). Sin embargo, no se puede predecir el valor de este alargamiento porque depende de cómo haya sido manipulado el tejido en el proceso de fabricación del laminado, y su efecto se reduce mucho con profundidades de pasada pequeñas.

$$
\text { hmax }>a_{p} \quad \text { (ecuación 4.14) }
$$

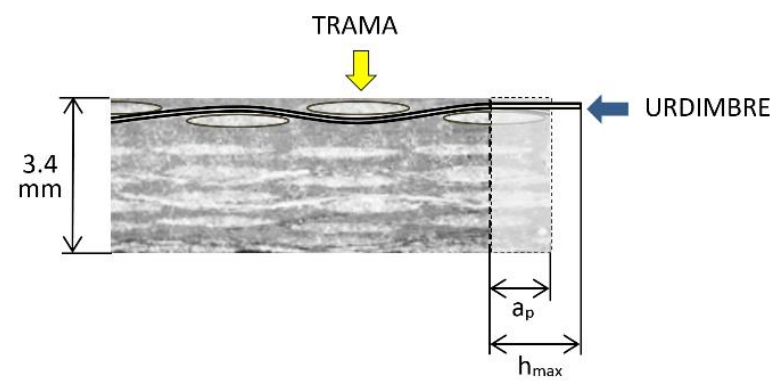

Figura 4.42. Corte transversal del tejido, $a_{p}$ y $h_{\max }$ observada

Si las profundidades de pasada son pequeñas, del orden de $0.5 \mathrm{~mm}$, se ha observado que se produce un delaminado muy pequeño. En este caso, aunque aparezca delaminado tipo II sobre el borde no supone un problema para la manipulación y el ensamblado de la pieza (Figura 4.44). 


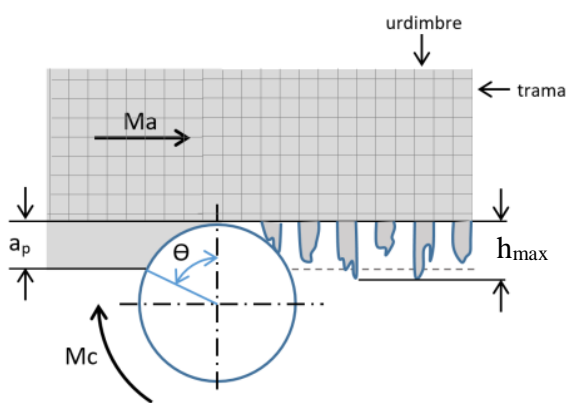

(a) $\mathrm{Fo}=0^{\circ}-90^{\circ}$

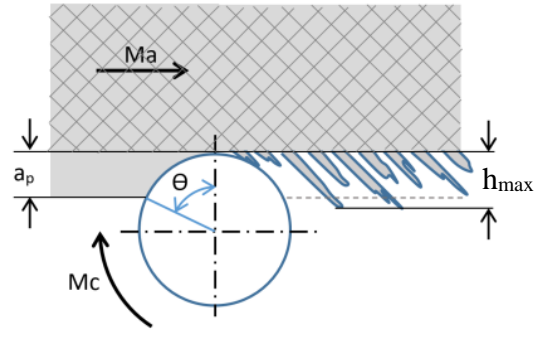

(b) $\mathrm{Fo}=45^{\circ}-135^{\circ}$

Figura 4.43. Valor del parámetro $h_{\max }$ sobre el tejido

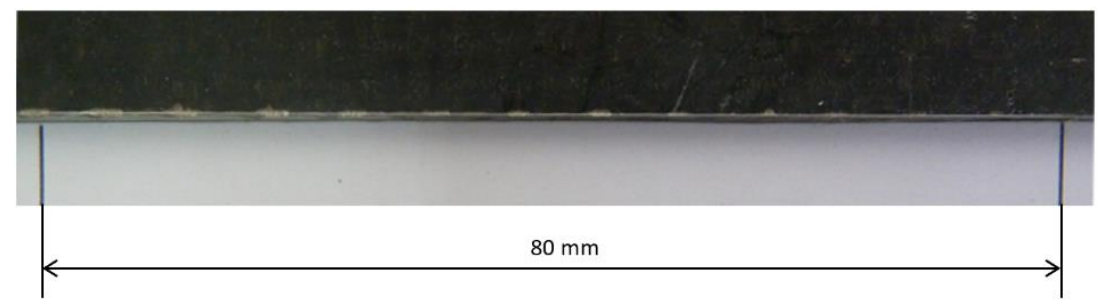

Figura 4.44. Delaminado observado en E05 $a_{p}=0.5 \mathrm{~mm}$ y $T c=80$ minutos

La profundidad de pasada $\left(\mathrm{a}_{\mathrm{p}}\right)$ es uno de los parámetros más importantes en el estudio del delaminado. La Tabla 4.7 compara de los parámetros Wa, $\mathrm{Wb}$ y Fdel entre experimentos con distinta profundidad de pasada $\left(\mathrm{a}_{\mathrm{p}}\right)$. Observando que una profundidad de pasada grande provoca un delaminado mayor en la pieza. Este efecto no se aprecia en el factor Fdel ya que este factor se ha calculado como la ratio respecto al valor teórico máximo esperado. De esta forma, tenemos una referencia común en todos los experimentos, mitigando el efecto de la profundidad de pasada para poder observar cómo afectan otros parámetros [78].

Tabla 4.7. Comparación de experimentos con distintas profundidades de pasada

\begin{tabular}{|c|c|c|c|}
\hline $\mathrm{ap}(\mathrm{mm})$ & $\overline{\mathrm{W}_{\mathrm{a}}}(\mathrm{mm})$ & $\overline{\mathrm{W}_{\mathrm{b}}}(\mathrm{mm})$ & $\overline{\mathrm{F}_{\mathrm{del}}}$ \\
\hline 0.5 & 0.086 & 0.221 & 0.441 \\
\hline \multirow[t]{3}{*}{1.5} & 0.373 & 0.751 & 0.500 \\
\hline & $\overline{\mathrm{W}_{\mathrm{a}}} a_{p} 1.5$ & $\overline{\mathrm{W}_{\mathrm{b}}} a_{p} 1.5$ & $\overline{\mathrm{F}_{\mathrm{del}}} a_{p} 1.5$ \\
\hline & $\overline{\mathrm{W}_{\mathrm{a}}} a_{p} 0.5$ & $\overline{\mathrm{W}_{\mathrm{b}}} a_{p} 0.5$ & $\overline{\mathrm{F}_{\mathrm{del}}} a_{p} 0.5$ \\
\hline
\end{tabular}




\subsubsection{PARÁMETROS DE SUPERFICIE}

Los parámetros unidimensionales sólo dan una estimación de la altura delaminada respecto al borde mecanizado. Sin embargo, el delaminado puede ser muy ligero, o puede invadir una zona de mayor superficie, presentándose un delaminado denso. Desde este punto de vista, los parámetros unidimensionales dan una aproximación muy limitada, ya que, por ejemplo, el valor de Wa y Wb para los dos casos que se presentan en la Figura 4.45 sería similar. Por este motivo, se va a definir un parámetro que además de la altura, tenga en cuenta la superficie delaminada (Sdi) de cada mecha.

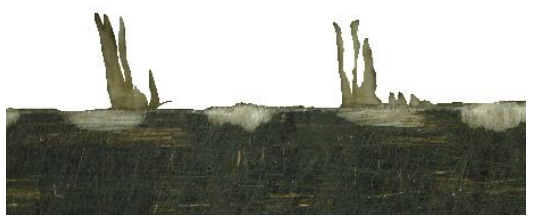

(a) ligero

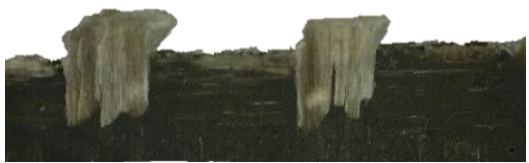

(b) denso

Figura 4.45. Delaminado tipo I/II: ligero y denso

\subsubsection{Factor de superficie (FSd)}

En primer lugar, se ha calculado un valor de superficie delaminada ( $\mathrm{Sd})$ para cada experimento. Este valor es una de las medidas realizadas que caracterizan el experimento. Corresponde al área delaminada en la longitud evaluada (L) (ecuación 4.5).

Para cuantificar la densidad del delaminado en cada experimento, se define un factor de superficie (FSd) que permite conocer la superficie promedio de las mechas.

El factor de superficie (FSd) se define como el cociente entre el área delaminada $(\mathrm{Sd})$, respecto al área delaminada máxima teórica $\left(\mathrm{Sd}_{\max }\right)$ para cada experimento (ecuación 4.15). Este factor permite realizar una comparación entre experimentos con distintas profundidades de pasada.

$$
F S d=\frac{S d}{S d \max }
$$

(ecuación 4.15)

$\mathrm{Al}$ igual que sucedía en los parámetros unidimensionales, en los tejidos con orientación $\mathrm{Fo}=45^{\circ}-135^{\circ}$, todas las mechas pasan por encima de la trama y tienen las mismas condiciones de disposición del tejido, por lo que con el cálculo de este factor (FSd) es suficiente y se evalúa bien el delaminado. Sin embargo, en tejidos con orientación $\mathrm{Fo}=0^{\circ}-90^{\circ}$ es necesario definir un factor 
distinto para las mechas que van por encima o por debajo de la trama, ya que suelen tener delaminados de distinto valor.

Para las mechas de tejido que sobresalen por debajo de la trama, en tejidos con orientación $\mathrm{Fo}=0^{\circ}-90^{\circ}$, se define $\mathrm{FSd}$ ' que considera sólo la superficie delaminada de las mechas que van por debajo de la trama (Sd'), (ecuación 4.16).

$$
F S d^{\prime}=\frac{S d^{\prime}}{S d \max } \quad \quad \text { (ecuación 4.16) }
$$

\subsection{Valor máximo teórico de la superficie delaminada $\left(S d_{\max }\right)$}

Como referencia para el cálculo del factor de delaminado de superficie (FSd) se han tomado los valores máximos teóricos de la superficie delaminada $\left(\mathrm{Sd}_{\max }\right)$.

En el caso del tejido con orientación $\mathrm{Fo}=0^{\circ}-90^{\circ}$, y para el tipo II de delaminado, esta superficie máxima es la de un rectángulo de base el ancho de mecha $(m)$ y altura la profundidad de pasada $\left(a_{p}\right)$ (Figura $4.46(a)$ ). Para el delaminado tipo I, se considera que la máxima superficie delaminada, es el área de la zona delimitada por el entrecruzado del tejido, cuyo lado corresponde con el ancho de la mecha de tejido $(3 \mathrm{~mm})$, considerando el valor máximo cuando la trayectoria de corte coincide con el cruce entre dos mechas (ecuaciones 4.17 y 4.18 ).

En el caso del tejido con orientación $\mathrm{Fo}=45^{\circ}-135^{\circ}$, el valor máximo de la superficie delaminada tipo II para una mecha de tejido, corresponde a un rectángulo de base la diagonal de una mecha de tejido de lado $3 \mathrm{~mm}$ (diagonal $=4.24 \mathrm{~mm}$ ) y altura la profundidad de pasada $\left(\mathrm{a}_{\mathrm{p}}\right)$ (Figura $4.46(\mathrm{~b})$ ). Para el delaminado tipo I es el área de la zona delimitada por el entrecruzado del tejido. Esto se puede extender a la longitud evaluada (L), calculando de esta forma las superficies teóricas máximas para el tipo de tejido utilizado en los experimentos (Tabla 4.8). Se utilizarán estos valores como referencia para cuantificar la presencia de delaminado en cada experimento.

$$
\begin{aligned}
& S d_{\max (1 m e c h a)}=\text { ancho mecha } a^{2} \quad \text { para Tipo I } \quad \text { (ecuación 4.17) } \\
& S d_{\max (1 \text { mecha })}=a_{p} \cdot m \quad \text { para Tipo II } \quad \text { (ecuación 4.18) }
\end{aligned}
$$

Siendo: $a_{\mathrm{p}}$ profundidad de pasada

m dimensión de la mecha de tejido, medida sobre la trayectoria de corte (base de la mecha delaminada) 


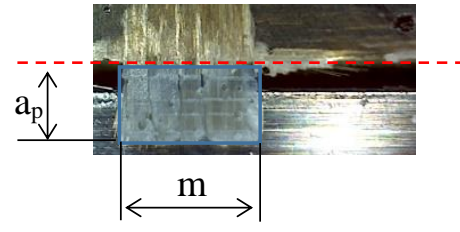

(a) Tejido Fo= $0^{\circ}-90^{\circ}$

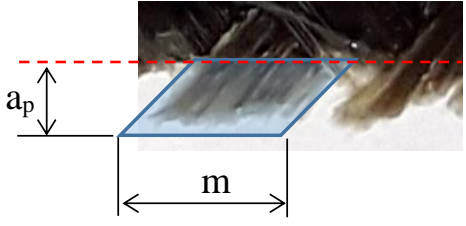

(b) Tejido Fo $=45^{\circ}-135^{\circ}$

Figura 4.46. Cálculo de Sd máxima teórica para delaminado Tipo II.

Tabla 4.8. Valores máximos teóricos de delaminado para una mecha y la longitud evaluada.

\begin{tabular}{cccccccc}
\hline Fo & $\begin{array}{c}\mathrm{ap} \\
\mathrm{mm}\end{array}$ & $\mathrm{m}$ & \multicolumn{2}{c}{$\mathrm{Sd}_{\max }(1 \mathrm{mecha})$} & $\mathrm{N}^{\circ}$ mechas & \multicolumn{2}{c}{$\mathrm{Sd}_{\max }(\mathrm{L}=80 \mathrm{~mm})$} \\
\cline { 4 - 8 } & $\mathrm{mm}$ & $\begin{array}{c}\mathrm{Tipo} \mathrm{I} \\
\mathrm{mm}^{2}\end{array}$ & $\begin{array}{c}\text { Tipo II } \\
\mathrm{mm}^{2}\end{array}$ & $\begin{array}{c}\mathrm{L}=80 \\
\mathrm{~mm}\end{array}$ & $\begin{array}{c}\text { Tipo I } \\
\mathrm{mm}^{2}\end{array}$ & $\begin{array}{c}\text { Tipo II } \\
\mathrm{mm}^{2}\end{array}$ \\
\hline \multirow{2}{*}{$0^{\circ}-90^{\circ}$} & 1.5 & 3 & 9 & 4.5 & 26.67 & 240 & 120 \\
& 0.5 & 3 & 9 & 1.5 & 26.67 & 240 & 40 \\
\hline \multirow{2}{*}{$45^{\circ}-135^{\circ}$} & 1.5 & 4.24 & 9 & 6.36 & 18.86 & 169.71 & 120 \\
& 0.5 & 4.24 & 9 & 2.12 & 18.86 & 169.71 & 40 \\
\hline
\end{tabular}

\subsubsection{Resultados obtenidos}

Las tablas 4.9 y 4.10 muestran los valores de superficie y los factores para ambos tipos de delaminado. Trabajar con áreas de delaminado permite sumar los factores FSd y FSd' en piezas con orientación de fibra de $\mathrm{Fo}=0^{\circ}-90^{\circ}$ para evaluar el área total delaminada, y poder comparar con experimentos con orientación de fibra de $\mathrm{Fo}=45^{\circ}-135^{\circ}$.

Tabla 4.9. Parámetros de superficie para delaminado Tipo I.

\begin{tabular}{ccccccccc}
\hline Exp & & $\begin{array}{c}\mathrm{Tc} \\
\min \end{array}$ & $\begin{array}{c}\mathrm{Sd} \\
\mathrm{mm}^{2}\end{array}$ & $\begin{array}{c}\mathrm{Sd}^{\prime} \\
\mathrm{mm}^{2}\end{array}$ & $\begin{array}{c}\mathrm{Sd}_{\max } \\
\mathrm{mm}^{2}\end{array}$ & FSd & FSd' & FSd + FSd' \\
\hline E05 & $\mathrm{Vc} 470$ & 30 & 1.79 & 0.21 & 240 & 0.0075 & 0.0009 & 0.0084 \\
& $\mathrm{Fz} 0.1$ & 50 & 2.48 & 0.63 & 240 & 0.0103 & 0.0026 & 0.0130 \\
& $\mathrm{a}_{\mathrm{p}} 0.5$ & 60 & 2.64 & 1.01 & 240 & 0.0110 & 0.0042 & 0.0152 \\
& Fo 90 & 70 & 1.97 & 1.83 & 240 & 0.0082 & 0.0076 & 0.0158 \\
& & 80 & 2.75 & 0.94 & 240 & 0.0114 & 0.0039 & 0.0153 \\
\hline E12 & $\mathrm{Vc} 300$ & 30 & 5.20 & 0.37 & 240 & 0.0217 & 0.0015 & 0.0232 \\
& Fz 0.4 & 50 & 3.82 & 0 & 240 & 0.0159 & 0 & 0.0159 \\
& $\mathrm{a}_{\mathrm{p}} 0.5$ & 60 & 4.21 & 0 & 240 & 0.0175 & 0 & 0.0175 \\
& Fo 90 & 70 & 4.98 & 0.31 & 240 & 0.0207 & 0.0013 & 0.0220 \\
& & 80 & 4.61 & 0.54 & 240 & 0.0192 & 0.0022 & 0.0215 \\
\hline
\end{tabular}




\begin{tabular}{|c|c|c|c|c|c|c|c|c|}
\hline Exp & & $\begin{array}{c}\mathrm{Tc} \\
\min \end{array}$ & $\begin{array}{c}\mathrm{Sd} \\
\mathrm{mm}^{2}\end{array}$ & $\begin{array}{c}\mathrm{Sd}^{\prime} \\
\mathrm{mm}^{2}\end{array}$ & $\begin{array}{l}\mathrm{Sd}_{\max } \\
\mathrm{mm}^{2}\end{array}$ & $\mathrm{FSd}$ & FSd' & $\mathrm{FSd}+\mathrm{FSd}$ \\
\hline \multirow[t]{5}{*}{ E11 } & Vc 300 & 30 & 6.46 & 0 & 240 & 0.0269 & 0 & 0.0269 \\
\hline & $\mathrm{Fz} 0.1$ & 50 & 7.42 & 0 & 240 & 0.0309 & 0 & 0.0309 \\
\hline & $a_{p} 1.5$ & 60 & 5.83 & 2.10 & 240 & 0.0243 & 0.0088 & 0.0331 \\
\hline & Fo $90^{\circ}$ & 70 & 3.89 & 1.96 & 240 & 0.0162 & 0.0082 & 0.0244 \\
\hline & & 80 & 5.07 & 4.29 & 240 & 0.0211 & 0.0179 & 0.0390 \\
\hline \multirow[t]{7}{*}{ E14 } & Vc 470 & 1.1 & 14.65 & 0.84 & 240 & 0.0611 & 0.0035 & 0.0646 \\
\hline & Fz 0.4 & 5 & 11.74 & 9.94 & 240 & 0.0489 & 0.0414 & 0.0903 \\
\hline & $a_{p} 1.5$ & 10 & 17.77 & 12.34 & 240 & 0.0740 & 0.0514 & 0.1254 \\
\hline & Fo $90^{\circ}$ & 20 & 28.08 & 7.55 & 240 & 0.1170 & 0.0315 & 0.1485 \\
\hline & & 31 & 26.46 & 4.33 & 240 & 0.1102 & 0.0180 & 0.1283 \\
\hline & & 37.5 & 12.87 & 0.94 & 240 & 0.0536 & 0.0039 & 0.0575 \\
\hline & & 40 & 6.15 & 25.46 & 240 & 0.0256 & 0.1061 & 0.1317 \\
\hline \multirow[t]{5}{*}{ E07 } & Vc 300 & 30 & 0.59 & & 169.71 & 0.0035 & & 0.0035 \\
\hline & $\mathrm{Fz} 0.1$ & 50 & 0.43 & & 169.71 & 0.0025 & & 0.0025 \\
\hline & $a_{p} 0.5$ & 60 & 1.35 & & 169.71 & 0.0079 & & 0.0079 \\
\hline & Fo $45^{\circ}$ & 70 & 0 & & 169.71 & 0 & & 0 \\
\hline & & 80 & 2.01 & & 169.71 & 0.0118 & & 0.0118 \\
\hline \multirow[t]{5}{*}{ E08 } & Vc 470 & 30 & 10.1 & & 169.71 & 0.0595 & & 0.0595 \\
\hline & Fz 0.4 & 50 & 9.65 & & 169.71 & 0.0569 & & 0.0569 \\
\hline & $a_{p} 0.5$ & 60 & 7.86 & & 169.71 & 0.0463 & & 0.0463 \\
\hline & Fo $45^{\circ}$ & 70 & 9.79 & & 169.71 & 0.0577 & & 0.0577 \\
\hline & & 80 & 2.58 & & 169.71 & 0.0152 & & 0.0152 \\
\hline \multirow[t]{5}{*}{ E04 } & Vc 300 & 30 & 13.09 & & 169.71 & 0.0772 & & 0.0772 \\
\hline & Fz 0.4 & 50 & 8.37 & & 169.71 & 0.0493 & & 0.0493 \\
\hline & $a_{p} 1.5$ & 60 & 10.96 & & 169.71 & 0.0646 & & 0.0646 \\
\hline & Fo $45^{\circ}$ & 70 & 8.35 & & 169.71 & 0.0492 & & 0.0492 \\
\hline & & 80 & 12.23 & & 169.71 & 0.0721 & & 0.0721 \\
\hline \multirow[t]{5}{*}{ E16 } & Vc 470 & 30 & 3.12 & & 169.71 & 0.0184 & & 0.0184 \\
\hline & Fz 0.1 & 50 & 9.64 & & 169.71 & 0.0568 & & 0.0568 \\
\hline & $\mathrm{a}_{\mathrm{p}} 1.5$ & 60 & 5.02 & & 169.71 & 0.0296 & & 0.0296 \\
\hline & Fo $45^{\circ}$ & 70 & 2.75 & & 169.71 & 0.0162 & & 0.0162 \\
\hline & & 80 & 6.85 & & 169.71 & 0.0403 & & 0.0403 \\
\hline
\end{tabular}


Tabla 4.10. Parámetros de superficie para delaminado Tipo II.

\begin{tabular}{|c|c|c|c|c|c|c|c|c|}
\hline Exp & & $\begin{array}{c}\mathrm{Tc} \\
\min \end{array}$ & $\begin{array}{c}\mathrm{Sd} \\
\mathrm{mm}^{2}\end{array}$ & $\begin{array}{l}\mathrm{Sd}^{\prime} \\
\mathrm{mm}^{2}\end{array}$ & $\begin{array}{l}\mathrm{Sd}_{\max } \\
\mathrm{mm}^{2}\end{array}$ & $\mathrm{FSd}$ & FSd' & $\mathrm{FSd}+\mathrm{FSd}$ \\
\hline \multirow[t]{5}{*}{ E05 } & Vc 470 & 30 & 5.19 & 1.17 & 40 & 0.1297 & 0.0292 & 0.1588 \\
\hline & $\mathrm{Fz} 0.1$ & 50 & 5.30 & 0.56 & 40 & 0.1324 & 0.0139 & 0.1464 \\
\hline & $a_{p} 0.5$ & 60 & 4.56 & 4.99 & 40 & 0.1141 & 0.1125 & 0.2266 \\
\hline & Fo $90^{\circ}$ & 70 & 8.42 & 7.27 & 40 & 0.2105 & 0.1818 & 0.3922 \\
\hline & & 80 & 4.12 & 4.94 & 40 & 0.1030 & 0.1025 & 0.2055 \\
\hline \multirow[t]{5}{*}{ E12 } & Vc 300 & 30 & 12.33 & 6.03 & 40 & 0.3084 & 0.1507 & 0.4590 \\
\hline & Fz 0.4 & 50 & 5.35 & 1.76 & 40 & 0.1338 & 0.0441 & 0.1779 \\
\hline & $\mathrm{a}_{\mathrm{p}} 0.5$ & 60 & 5.35 & 0.70 & 40 & 0.1338 & 0.0175 & 0.1513 \\
\hline & Fo $90^{\circ}$ & 70 & 5.39 & 0.79 & 40 & 0.1346 & 0.0198 & 0.1544 \\
\hline & & 80 & 9.03 & 9.37 & 40 & 0.2257 & 0.2250 & 0.4507 \\
\hline \multirow[t]{5}{*}{ E11 } & Vc 300 & 30 & 8.68 & 1.96 & 120 & 0.0724 & 0.0163 & 0.0887 \\
\hline & Fz 0.1 & 50 & 23.35 & 1.24 & 120 & 0.1946 & 0.0104 & 0.2050 \\
\hline & $\mathrm{a}_{\mathrm{p}} 1.5$ & 60 & 42.40 & 8.18 & 120 & 0.3533 & 0.0682 & 0.4215 \\
\hline & Fo $90^{\circ}$ & 70 & 21.99 & 8.27 & 120 & 0.1832 & 0.0690 & 0.2522 \\
\hline & & 80 & 22.08 & 5.58 & 120 & 0.1840 & 0.0465 & 0.2305 \\
\hline \multirow[t]{7}{*}{ E14 } & Vc 470 & 1.1 & 23.41 & 7.95 & 120 & 0.1951 & 0.0662 & 0.2614 \\
\hline & Fz 0.4 & 5 & 51.42 & 14.29 & 120 & 0.4285 & 0.1191 & 0.5476 \\
\hline & $a_{p} 1.5$ & 10 & 43.04 & 29.41 & 120 & 0.3586 & 0.2451 & 0.6037 \\
\hline & Fo $90^{\circ}$ & 20 & 37.10 & 16.10 & 120 & 0.3091 & 0.1341 & 0.4433 \\
\hline & & 31 & 41.21 & 17.77 & 120 & 0.3434 & 0.1481 & 0.4915 \\
\hline & & 37.5 & 38.73 & 13.28 & 120 & 0.3228 & 0.1106 & 0.4334 \\
\hline & & 40 & 67.29 & 24.60 & 120 & 0.5608 & 0.2050 & 0.7658 \\
\hline \multirow[t]{5}{*}{ E07 } & Vc 300 & 30 & 2.23 & & 40 & 0.0558 & & 0.0558 \\
\hline & Fz 0.1 & 50 & 1.68 & & 40 & 0.0420 & & 0.0420 \\
\hline & $\mathrm{a}_{\mathrm{p}} 0.5$ & 60 & 0.68 & & 40 & 0.0171 & & 0.0171 \\
\hline & Fo $45^{\circ}$ & 70 & 2.37 & & 40 & 0.0593 & & 0.0593 \\
\hline & & 80 & 3.12 & & 40 & 0.0780 & & 0.0780 \\
\hline \multirow[t]{5}{*}{ E08 } & Vc 470 & 30 & 15.15 & & 40 & 0.3788 & & 0.3788 \\
\hline & $\mathrm{Fz} 0.4$ & 50 & 13.23 & & 40 & 0.3308 & & 0.3308 \\
\hline & $\mathrm{a}_{\mathrm{p}} 0.5$ & 60 & 10.87 & & 40 & 0.2718 & & 0.2718 \\
\hline & Fo $45^{\circ}$ & 70 & 13.4 & & 40 & 0.3350 & & 0.3350 \\
\hline & & 80 & 10.2 & & 40 & 0.2550 & & 0.2550 \\
\hline
\end{tabular}




\begin{tabular}{|c|c|c|c|c|c|c|c|c|}
\hline \multirow{2}{*}{ Exp } & & $\mathrm{Tc}$ & $\mathrm{Sd}$ & $\mathrm{Sd}$ ' & $\mathrm{Sd}_{\max }$ & \multirow{2}{*}{$\mathrm{FSd}$} & \multirow{2}{*}{ FSd' } & \multirow{2}{*}{$\mathrm{FSd}+\mathrm{FSd}$} \\
\hline & & $\min$ & $\mathrm{mm}^{2}$ & $\mathrm{~mm}^{2}$ & $\mathrm{~mm}^{2}$ & & & \\
\hline \multirow[t]{5}{*}{ E04 } & Vc 300 & 30 & 29.77 & & 120 & 0.2481 & & 0.2481 \\
\hline & $\mathrm{Fz} 0.4$ & 50 & 39.85 & & 120 & 0.3320 & & 0.3320 \\
\hline & $a_{p} 1.5$ & 60 & 47.71 & & 120 & 0.3976 & & 0.3976 \\
\hline & Fo $45^{\circ}$ & 70 & 23.42 & & 120 & 0.1951 & & 0.1951 \\
\hline & & 80 & 47.73 & & 120 & 0.3978 & & 0.3978 \\
\hline \multirow[t]{5}{*}{ E16 } & Vc 470 & 30 & 30.93 & & 120 & 0.2578 & & 0.2578 \\
\hline & $\mathrm{Fz} 0.1$ & 50 & 19.87 & & 120 & 0.1656 & & 0.1656 \\
\hline & $a_{p} 1.5$ & 60 & 51.29 & & 120 & 0.4274 & & 0.4274 \\
\hline & Fo $45^{\circ}$ & 70 & 18.41 & & 120 & 0.1534 & & 0.1534 \\
\hline & & 80 & 19.57 & & 120 & 0.1631 & & 0.1631 \\
\hline
\end{tabular}

\subsubsection{Rangos de delaminado para el factor de superficie}

Se establece un rango de variación del delaminado para FSd y FSd' en cada experimento para el delaminado tipo I y II (Figuras 4.47 y 4.48 ).

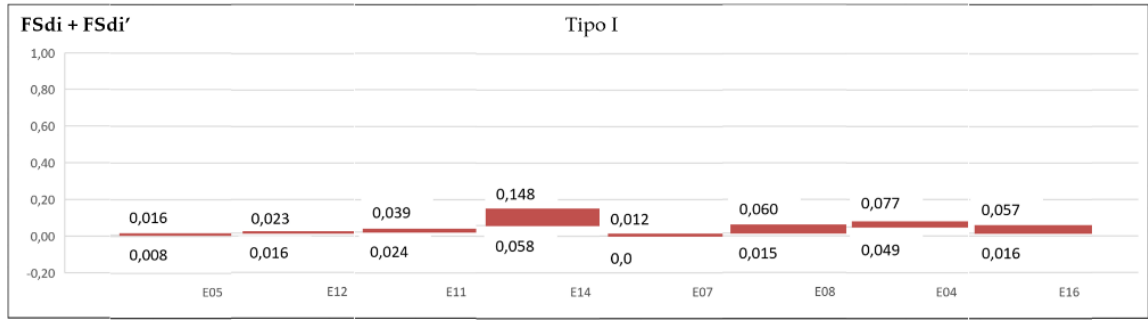

Figura 4.47. Rango para el Factor de superficie de delaminado Tipo I (FSd+FSd').

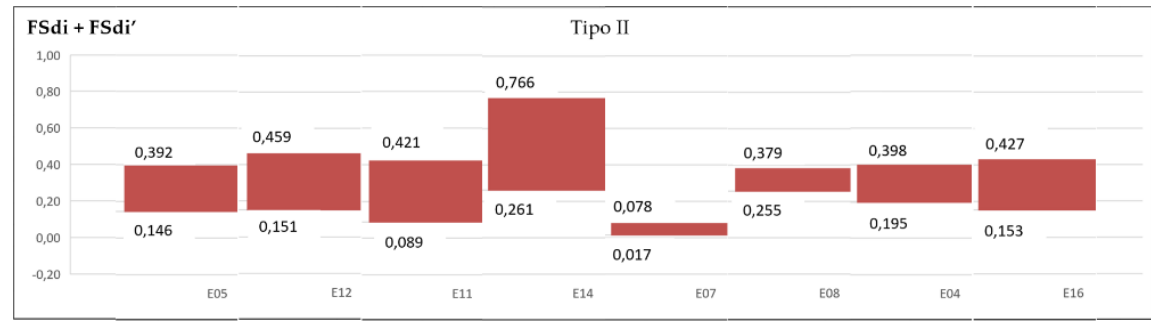

Figura 4.48. Rango para el Factor de superficie de delaminado Tipo II (FSd+FSd'). 


\subsection{ANÁLISIS ESTADÍSTICO}

En esta sección se presentan los resultados del Análisis de Varianza (ANOVA) para analizar el efecto de las condiciones de mecanizado y de la orientación de la fibra en la superficie delaminada de los experimentos desarrollados. Adicionalmente, se ha analizado la relación existente entre los diferentes parámetros que permiten medir el delaminado mediante un análisis de correlación. En los siguientes apartados se muestran los resultados obtenidos para los dos tipos de delaminado estudiados (tipo I y II).

\subsubsection{DELAMINADO TIPO I}

El objetivo de este apartado se centra, como se ha indicado previamente, en analizar si existen diferencias estadísticamente significativas en las medias de los diferentes parámetros que miden el delaminado (Wa, Wb, Sd y FSd+FSd') considerando como factores las condiciones de mecanizado ( $\mathrm{Vc}, \mathrm{Fz}$ y $\mathrm{a}_{\mathrm{p}}$ ) y la orientación de la fibra $(\mathrm{Fo})$. Para ello, se ha realizado un ANOVA paramétrico cuyos resultados se muestran en las Tablas 4.11, 4.12, 4.13 y 4.14.

Tabla 4.11. Análisis de varianza (ANOVA) Wa Tipo I.

\begin{tabular}{cccccc}
\hline Factor & Df & Sum Sq & Mean Sq & F Value & $\operatorname{Pr}(>\mathrm{F})$ \\
\hline Vc & 1 & $1.6 \mathrm{E}-03$ & $1.6 \mathrm{E}-03$ & 2.45 & 0.128 \\
$\mathrm{Fz}$ & 1 & 0.0285 & 0.0285 & 43.37 & $1.35 \mathrm{E}-06^{*}$ \\
$\mathrm{a}_{\mathrm{p}}$ & 1 & 0.0200 & 0.0200 & 30.45 & $8.01 \mathrm{E}-07^{*}$ \\
$\mathrm{Fo}$ & 1 & $2.1 \mathrm{E}-04$ & $2.1 \mathrm{E}-04$ & 0.32 & 0.127 \\
Residuos & 30 & 0.0197 & $6.6 \mathrm{E}-04$ & & \\
\hline
\end{tabular}

Tabla 4.12. Análisis de varianza (ANOVA) Wb Tipo I.

\begin{tabular}{cccccc}
\hline Factor & Df & Sum Sq & Mean Sq & F Value & $\operatorname{Pr}(>\mathrm{F})$ \\
\hline Vc & 1 & $4.40 \mathrm{E}-04$ & $4.40 \mathrm{E}-04$ & 0.15 & 0.6992 \\
$\mathrm{Fz}$ & 1 & 0.05896 & 0.05896 & 20.36 & $1.00 \mathrm{E}-04^{*}$ \\
$\mathrm{a}_{\mathrm{p}}$ & 1 & 0.03234 & 0.03234 & 11.17 & $2.22 \mathrm{E}-03^{*}$ \\
Fo & 1 & $1.33 \mathrm{E}-03$ & $1.33 \mathrm{E}-03$ & 0.46 & 0.5027 \\
Residuos & 30 & 0.08546 & $2.85 \mathrm{E}-03$ & & \\
\hline
\end{tabular}

Tabla 4.13. Análisis de varianza (ANOVA) Sd Tipo I.

\begin{tabular}{cccccc}
\hline Factor & Df & Sum Sq & Mean Sq & F Value & $\operatorname{Pr}(>\mathrm{F})$ \\
\hline Vc & 1 & 13.57 & 13.57 & 3.340 & 0.077 \\
Fz & 1 & 189.45 & 189.45 & 46.680 & $<1 \mathrm{E}-05^{*}$ \\
ap & 1 & 141.98 & 141.98 & 34.990 & $<1 \mathrm{E}-05^{*}$ \\
Fo & 1 & 0.44 & 0.44 & 0.110 & 0.744 \\
Residuos & 30 & 121.75 & 4.06 & & \\
\hline
\end{tabular}


Tabla 4.14. Análisis de varianza (ANOVA) FSd+FSd'Tipo I.

\begin{tabular}{cccccc}
\hline Factor & Df & Sum Sq & Mean Sq & F Value & $\operatorname{Pr}(>\mathrm{F})$ \\
\hline Vc & 1 & $1.20 \mathrm{E}-03$ & $1.2 \mathrm{E}-03$ & 8.05 & $0.0081^{*}$ \\
Fz & 1 & $6.16 \mathrm{E}-03$ & $6.16 \mathrm{E}-03$ & 41.21 & $<1 \mathrm{E}-05^{*}$ \\
ap & 1 & $4.94 \mathrm{E}-03$ & $4.94 \mathrm{E}-03$ & 33.04 & $<1 \mathrm{E}-05^{*}$ \\
Fo & 1 & $1.41 \mathrm{E}-04$ & $1.41 \mathrm{E}-04$ & 0.94 & 0.3396 \\
Residuos & 30 & $1.54 \mathrm{E}-02$ & $1.50 \mathrm{E}-4$ & & \\
\hline
\end{tabular}

Los resultados del análisis de varianza mostrados en la Tabla 4.11 confirman que existe una diferencia estadísticamente significativa entre las medias del delaminado medio, Wa, para los diferentes niveles de Fz y a valor $<0.05)$ no siendo significativo el efecto de las variables Vc y Fo. Las mismas conclusiones pueden obtenerse, a partir de las Tabla 4.12 y Tabla 4.13, en el caso del delaminado observado, Wb, y la superficie delaminada, Sd. Finalmente, en el caso del factor de delaminado FSd+FSd', se puede concluir que existen tres factores estadísticamente significativos ( $\mathrm{Vc}, \mathrm{Fz}$ y $\mathrm{a}_{\mathrm{p}}$ ). Así, analizando los resultados de la Tabla 4.14 se observa que el avance por diente (Fz) y el $a_{p}$ son los parámetros más significativos, seguidos de la velocidad de corte $(\mathrm{Vc})$. La orientación de la fibra (Fo) no parece influir en el factor de delaminado. Estas conclusiones que son acordes a lo esperado, ya que un avance por diente menor, implica un mayor número de impactos de la herramienta en el ancho de la mecha que reduce el valor de la superficie delaminada.

La validez de los resultados obtenidos del ANOVA depende del cumplimiento de los supuestos del análisis (normalidad, homocedasticidad e independencia). Para contrastar la normalidad se utiliza el test de Saphiro-Wilk cuyos resultados se muestran en la Tabla 4.15. En todos los casos se obtiene un p-valor mayor de 0.05 , por tanto, se puede concluir que los datos cumplen el supuesto de normalidad.

Tabla 4.15. Resultado test de Saphiro-Wilk.

\begin{tabular}{ccc}
\hline Parámetro & Estadístico & p-valor \\
\hline $\mathrm{Wa}$ & 0.971 & 0.456 \\
$\mathrm{~Wb}$ & 0.976 & 0.643 \\
$\mathrm{Sd}$ & 0.967 & 0.364 \\
FSd + FSd' & 0.963 & 0.275 \\
\hline
\end{tabular}

Para contrastar el supuesto de homocedasticidad se ha seleccionado el test de Levene. La Tabla 4.16 muestra los resultados obtenidos observándose que el p-valor, en todos los casos, es menor de 0.05 existiendo evidencia estadística para rechazar el supuesto de igualdad de homogeneidad de varianzas. 
Tabla 4.16. Resultado test de Levene.

\begin{tabular}{ccccc}
\hline Parámetro & F & df1 & df2 & p-valor \\
\hline Wa & 7.98 & 3 & 31 & $<0.001$ \\
Wb & 4.13 & 3 & 31 & 0.014 \\
Sdi & 6.60 & 3 & 31 & 0.001 \\
FSd + FSd & 5.31 & 3 & 31 & 0.005 \\
\hline
\end{tabular}

Como consecuencia del incumplimiento de los supuestos del ANOVA paramétrico se ha procedido a realizar un ANOVA robusto cuyos resultados, para el delaminado tipo I, se muestran en la Tabla 4.17. Si se comparan los resultados obtenidos con el ANOVA paramétrico y el robusto se observa que, en el caso del valor medio de Wb y FSd+FSd', el resultado de ambas pruebas coincide. Sin embargo, a partir de los resultados del ANOVA robusto se concluye que existe una diferencia estadísticamente significativa en los valores medios de Wa y Sdi, ya que además de depender de la Fz y la ap, dependen también de la Vc, factor que no era estadísticamente significativo en el ANOVA paramétrico.

Tabla 4.17. Análisis de varianza (ANOVA) robusto delaminado Tipo I.

\begin{tabular}{ccccccccc}
\hline & \multicolumn{2}{c}{$\mathrm{Wa}$} & \multicolumn{2}{c}{$\mathrm{Wb}$} & \multicolumn{2}{c}{ Sdi } & \multicolumn{2}{c}{ FSd + FSd } \\
\hline Factor & $\mathrm{F}$ & $\operatorname{Pr}(>\mathrm{F})$ & $\mathrm{F}$ & $\operatorname{Pr}(>\mathrm{F})$ & $\mathrm{F}$ & $\operatorname{Pr}(>\mathrm{F})$ & $\mathrm{F}$ & $\operatorname{Pr}(>\mathrm{F})$ \\
\hline $\mathrm{Vc}$ & 4.85 & $0.03^{*}$ & 0.08 & 0.77 & 4.83 & $0.03^{*}$ & 6.77 & $0.01^{*}$ \\
$\mathrm{Fz}$ & 47.16 & $1.22 \mathrm{E}-07^{*}$ & 17.37 & $2.40 \mathrm{E}-04^{*}$ & 47.13 & $1.3 \mathrm{E}-07^{*}$ & 39.27 & $6.6 \mathrm{E}-07^{*}$ \\
$\mathrm{a}_{\mathrm{p}}$ & 34.81 & $1.84 \mathrm{E}-06^{*}$ & 10.02 & $3.53 \mathrm{E}-03^{*}$ & 34.57 & $1.95 \mathrm{E}-06^{*}$ & 34.73 & $1.8 \mathrm{E}-06^{*}$ \\
$\mathrm{Fo}$ & 0.27 & 0.61 & 0.32 & 0.57 & 0.28 & 0.60 & 1.77 & 0.19 \\
\hline
\end{tabular}

\subsubsection{DELAMINADO TIPO II}

De forma similar al apartado anterior se ha analizado si existen diferencias estadísticamente significativas en las medias de los diferentes parámetros (Wa, $\mathrm{Wb}, \mathrm{Sd}$ y FSd+FSd') que miden el delaminado tipo II considerando los mismos factores, es decir, tanto las condiciones de mecanizado ( $\mathrm{Vc}, \mathrm{Fz}$ y $\mathrm{a}_{\mathrm{p}}$ ) como la orientación de la fibra (Fo). Los resultados del ANOVA paramétrico se muestran en las Tablas 4.18, 4.19, 4.20 y 4.21.

Tabla 4.18. Análisis de varianza (ANOVA) Wa Tipo II.

\begin{tabular}{cccccc}
\hline Factor & Df & Sum Sq & Mean Sq & F Value & $\operatorname{Pr}(>\mathrm{F})$ \\
\hline Vc & 1 & 0.01008 & 0.01008 & 0.96 & 0.3343 \\
Fz & 1 & 0.09020 & 0.09020 & 8.61 & $0.0063^{*}$ \\
ap & 1 & 0.70074 & 0.70074 & 66.92 & $<1 \mathrm{E}-05^{*}$ \\
Fo & 1 & $4.08 \mathrm{E}-03$ & $4.08 \mathrm{E}-03$ & 0.39 & 0.5370 \\
Residuos & 30 & 0.31413 & 0.01047 & & \\
\hline
\end{tabular}


Tabla 4.19. Análisis de varianza (ANOVA) Wb Tipo II.

\begin{tabular}{cccccc}
\hline Factor & Df & Sum Sq & Mean Sq & F Value & $\operatorname{Pr}(>\mathrm{F})$ \\
\hline Vc & 1 & $8.17 \mathrm{E}-03$ & $8.17 \mathrm{E}-03$ & 0.24 & 0.6253 \\
$\mathrm{Fz}$ & 1 & 0.14077 & 0.14077 & 4.20 & $0.0493^{*}$ \\
$\mathrm{ap}$ & 1 & 2.31019 & 2.31019 & 68.88 & $<1 \mathrm{E}-05^{*}$ \\
Fo & 1 & $1.69 \mathrm{E}-03$ & $1.69 \mathrm{E}-03$ & 0.05 & 0.8240 \\
Residuos & 30 & 1.00616 & 0.03354 & & \\
\hline
\end{tabular}

Tabla 4.20. Análisis de varianza (ANOVA) Sd Tipo II.

\begin{tabular}{cccccc}
\hline Factor & Df & Sum Sq & Mean Sq & F Value & $\operatorname{Pr}(>\mathrm{F})$ \\
\hline Vc & 1 & 62.136 & 62.136 & 0.93 & 0.3413 \\
Fz & 1 & 568.371 & 568.371 & 8.55 & $6.5 \mathrm{E}-03^{*}$ \\
$\mathrm{a}_{\mathrm{p}}$ & 1 & 4513.77 & 4513.77 & 67.91 & $<1 \mathrm{E}-05^{*}$ \\
Fo & 1 & 24.8066 & 24.8066 & 0.37 & 0.5458 \\
Residuos & 30 & 1993.93 & 66.4642 & & \\
\hline
\end{tabular}

Tabla 4.21. Análisis de varianza (ANOVA) FSd+Fsd'Tipo II.

\begin{tabular}{cccccc}
\hline Factor & Df & Sum Sq & Mean Sq & F Value & $\operatorname{Pr}(>\mathrm{F})$ \\
\hline Vc & 1 & 0.06721 & 0.06721 & 6.69 & $0.0148^{*}$ \\
Fz & 1 & 0.19360 & 0.19360 & 19.26 & $1 \mathrm{E}-04^{*}$ \\
$\mathrm{ap}_{\mathrm{p}}$ & 1 & 0.07450 & 0.07450 & 7.41 & $0.0107^{*}$ \\
Fo & 1 & 0.04524 & 0.04524 & 4.50 & $0.0422^{*}$ \\
Residuos & 30 & 0.30149 & 0.01005 & & \\
\hline
\end{tabular}

Los resultados obtenidos del análisis de la varianza para el delaminado medio, Wa, el delaminado observado, $\mathrm{Wb}$, y la superficie delaminada, $\mathrm{Sd}$, en el caso del delaminado tipo II (Tablas 4.18, 4.19 y 4.20) son similares a los obtenidos en el apartado anterior observándose un efecto estadísticamente significativo de los factores Fz y a $a_{p}$ no siendo significativo el efecto de $\mathrm{Vc}$ y Fo. Sin embargo, los resultados mostrados en la Tabla 4.21 correspondientes al ANOVA para el factor de superficie, FSd+FSd', difieren de los obtenidos en el Tipo I, pudiéndose concluir que los cuatro factores analizados $\left(\mathrm{Vc}, \mathrm{Fz}, \mathrm{a}_{\mathrm{p}}\right.$ y Fo) son estadísticamente significativos.

El cumplimiento de los supuestos de normalidad y homocedasticidad se ha realizado a partir de los test de Saphiro-Wilk y el test de Levene, respectivamente. Los resultados de dichos tests se muestran en las Tablas 4.22 y 4.23. En la Tabla 4.22 se observa p-valores menores a 0.05 por lo que se incumple el supuesto de normalidad. 
Tabla 4.22. Resultado test de Saphiro-Wilk.

\begin{tabular}{ccc}
\hline Parámetro & Estadístico & p-valor \\
\hline $\mathrm{Wa}$ & 0.919 & 0.013 \\
$\mathrm{~Wb}$ & 0.957 & 0.187 \\
$\mathrm{Sd}$ & 0.921 & 0.015 \\
$\mathrm{FSd}+\mathrm{FSd}$, & 0.940 & 0.057 \\
\hline
\end{tabular}

Para contrastar el supuesto de homocedasticidad se ha seleccionado el test de Levene. La Tabla 4.23 muestra los resultados obtenidos observándose que el p-valor, en todos los casos, es menor de 0.05 existiendo evidencia estadística para rechazar el supuesto de igualdad de homogeneidad de varianzas.

Tabla 4.23. Resultado test de Levene.

\begin{tabular}{ccccc}
\hline Parámetro & F & df1 & df2 & p-valor \\
\hline Wa & 4.48 & 6 & 28 & 0.003 \\
Wb & 4.13 & 6 & 28 & 0.028 \\
Sd & 4.27 & 6 & 28 & 0.004 \\
FSd + FSd & 3.23 & 6 & 28 & 0.015 \\
\hline
\end{tabular}

Como consecuencia del incumplimiento de los supuestos del ANOVA paramétrico se ha procedido a realizar un ANOVA robusto cuyos resultados se muestran en la Tabla 4.24. Si se comparan los resultados se observa que coinciden los factores estadísticamente significativos para $\mathrm{Wa}$, Wb y Sdi. En el caso de FSd + FSd' los resultados del ANOVA robusto muestran un efecto estadísticamente significativo de los factores $\mathrm{Vc}, \mathrm{Fz}$ y $\mathrm{a}_{\mathrm{p}}$ no siendo significativo Fo.

Tabla 4.24. Análisis de varianza (ANOVA) robusto delaminado Tipo II

\begin{tabular}{ccccccccc}
\hline & \multicolumn{2}{c}{ Wa } & \multicolumn{2}{c}{ Wb } & \multicolumn{2}{c}{ Sdi } & \multicolumn{2}{c}{ FSd + FSd' } \\
\hline Factor & $\mathrm{F}$ & $\operatorname{Pr}(>\mathrm{F})$ & $\mathrm{F}$ & $\operatorname{Pr}(>\mathrm{F})$ & $\mathrm{F}$ & $\operatorname{Pr}(>\mathrm{F})$ & $\mathrm{F}$ & $\operatorname{Pr}(>\mathrm{F})$ \\
\hline $\mathrm{Vc}$ & 0.82 & 0.37 & 0.36 & 0.55 & 0.82 & 0.37 & 5.58 & $0.02^{*}$ \\
$\mathrm{Fz}$ & 14.56 & $6.3 \mathrm{E}-04^{*}$ & 5.97 & $0.02^{*}$ & 14.57 & $6.2 \mathrm{E}-04^{*}$ & 17.14 & $2.6 \mathrm{E}-04^{*}$ \\
$\mathrm{a}_{\mathrm{p}}$ & 82.65 & $4.01 \mathrm{E}-10^{*}$ & 84.57 & $3.10 \mathrm{E}-10^{*}$ & 82.60 & $4.03 \mathrm{E}-10^{*}$ & 6.21 & $1.8 \mathrm{E}-02^{*}$ \\
$\mathrm{Fo}$ & 0.34 & 0.56 & 0.17 & 0.68 & 0.34 & 0.56 & 3.48 & 0.07 \\
\hline
\end{tabular}




\subsection{CONCLUSIONES}

Las principales conclusiones relacionadas con el delaminado, tras los experimentos y los análisis estadísticos llevados a cabo, se muestran a continuación:

El delaminado en las capas exteriores sólo aparece en las piezas con una fracción de volumen de fibra (Fv) del 60\%. El delaminado tipo III es despreciable frente a los del tipo I y II. Analizando los parámetros unidimensionales, se puede concluir que el delaminado tipo II es más importante que el tipo I.

Los parámetros y factores de delaminado se calculan en diferentes tiempos de corte. Los valores de delaminado presentan una gran variación en estos tiempos de corte, sin una tendencia creciente o decreciente a medida que aumenta el tiempo de corte y el desgaste de la herramienta. Esta variación se debe a la naturaleza aleatoria de la delaminación. Para cada tiempo de corte, la distancia del hilo de urdimbre desde el borde recortado hasta la próxima inmersión por debajo del hilo de relleno cruzado (Xd) cambia, siendo éste un parámetro fundamental para evaluar el delaminado tipo II.

Los análisis de ANOVA robusto muestran que la profundidad de corte y el avance por diente son los factores más significativos en los parámetros unidimensionales (Wa y $\mathrm{Wb}$ ) y de superficie (Sd), tanto para el delaminado tipo I como para el tipo II. En relación al factor FSd+FSd', tanto para el tipo I como el tipo II son significativas las condiciones de corte.

El delaminado se ha medido en las mechas de urdimbre por encima y por debajo de la trama en laminados con orientación de fibra $\mathrm{Fo}=0^{\circ}-90^{\circ}$. En la mayoría de las mediciones realizadas para tejidos con orientación de fibra $\mathrm{Fo}=0^{\circ}-90^{\circ}$, el delaminado en las mechas que pasan por encima de la trama es mayor que en las mechas que pasan por debajo, se puede concluir que, en la mayoría de los casos, el delaminado de las mechas por debajo de la trama es despreciable. 


\section{CAPÍTULO 5. CONCLUSIONES Y TRABAJOS FUTUROS}

\subsection{CONCLUSIONES}

El mecanizado de materiales compuestos reforzados con tejido es un proceso complejo, estando los resultados condicionados por muchas variables. El análisis de los experimentos desarrollados en la tesis ha permitido entender cómo se produce el fenómeno del corte y estudiar, principalmente, el defecto del delaminado.

Las principales conclusiones y aportaciones derivadas de la tesis doctoral desarrollada se mencionan a continuación:

- En esta tesis se ha abordado por primera vez el fresado de materiales compuestos reforzados con fibra de basalto, siendo éste un material de origen natural y eco-friendly. Se observa un comportamiento similar en muchos aspectos a estudios publicados con fibras de carbono y vidrio, por lo que los resultados obtenidos en esta tesis se podrían extrapolar a estos otros materiales.

- La selección de la herramienta de mecanizado ha sido importante en el desarrollo de la tesis. La herramienta ha permitido mecanizar grandes longitudes, manteniendo un desgaste pequeño y presentando una gran productividad para el mecanizado de este tipo de materiales. 
- Los resultados del estudio de la influencia de los parámetros de corte en la medida de la rugosidad superficial ( $\mathrm{Ra}$ ) han sido consistentes con los análisis existentes en la bibliografía. El parámetro Ra aumenta su valor conforme el tiempo de corte y el desgate de la herramienta aumentan. Manteniéndose estos valores por debajo de las 10 micras, tras un tiempo de corte de 80 minutos en todos los experimentos realizados. Se concluye que cuando el tiempo de corte aumenta, las características del material tienen más influencia que las condiciones de mecanizado. Valores de $\mathrm{Fv}=60 \%$ y $\mathrm{Fo}=45^{\circ}$ dan peores resultados de rugosidad.

- En relación al desgaste de la herramienta, aún habiendo mecanizado durante 80 minutos, el desgaste de la herramienta se encuentra en la zona lineal, no habiendo alcanzado la zona de desgaste exponencial hasta perder la capacidad de corte. El desgaste ha sido uniforme en ambos filos, y aumenta siempre, conforme aumenta el tiempo de mecanizado. Los factores que influyen en el desgaste son el avance por diente y la velocidad de corte. Valores de $\mathrm{Fz}=0.4 \mathrm{~mm} /$ diente y $\mathrm{Vc}=470 \mathrm{~m} / \mathrm{min}$ dan un mayor desgaste de la herramienta de corte.

- En la tesis, para cuantificar el delaminado, se han definido tanto parámetros unidimensionales como de área para los tipos I y II de delaminado. Los unidimensionales $(\mathrm{Wa}, \mathrm{Wb})$ evalúan la longitud de las hebras, mientras que los de área ( $\mathrm{Sd}$ ) evalúan la densidad del delaminado. Con estos parámetros se ha constatado que el delaminado tipo II es más importante.

- En el estudio del delaminado, los parámetros de delaminado se han obtenido en diferentes tiempos de mecanizado para cada uno de los experimentos. No se aprecia una tendencia clara al aumento o disminución de dichos parámetros conforme el tiempo aumenta, no existiendo una correlación clara entre el delaminado y el desgaste de la herramienta. Se ha constatado que la predicción del delaminado no es fácil, debido a su naturaleza aleatoria. Esto es debido a que en cada instante de tiempo, la distancia del punto donde incide la herramienta sobre el laminado hasta el cruce de mechas de tejido más próximo es aleatoria. Por otro lado, el tejido puede estar ligeramente inclinado con respecto a la dirección de corte. Estos dos fenómenos pueden provocar varias formas en el aspecto del delaminado tipo II: uniforme, en forma de onda o totalmente aleatorio.

- En relación a los parámetros que afectan a la aparición del delaminado, el volumen de fibra es el parámetro más importante, ya que en laminados con un volumen de fibra $\mathrm{Fv}=40 \%$ no aparece delaminado, mientras que en todos los laminados con un volumen de fibra $\mathrm{Fv}=60 \%$ sí que lo presentan. Muchos autores se han centrado en el estudio del avance y la 
velocidad como parámetros de corte más importantes, sin considerar la profundidad de pasada. Sin embargo, la profundidad de pasada es la variable que tiene más influencia en el tamaño del delaminado tipo II.

- Por último, se han desarrollado modelos ANOVA para ver la influencia de los parámetros estudiados (condiciones de corte y características del material) en la medida de la rugosidad, el desgaste de la herramienta y el delaminado. Se han tratado los datos con un modelo de regresión cuantílica.

\subsection{TRABAJOS FUTUROS}

Como continuación de la presente Tesis Doctoral, se proponen las siguientes líneas de investigación:

- Debido al buen comportamiento de la herramienta seleccionada ante el desgaste, en cada experimento se ha mecanizado únicamente 80 minutos, estando la curva de desgaste en función del tiempo en la zona plana. Se propone continuar con los experimentos para terminar de caracterizar la curva de desgaste, incluso utilizando herramientas desgastadas previamente para agilizar los experimentos y terminar de evaluar el desgaste, la rugosidad y el delaminado con tiempos de corte mayores.

- Debido al alto coste de llevar a cabo los experimentos de mecanizado, se propone comenzar el desarrollo de modelos en 3D de la operación de contorneado del proceso de fresado mediante elementos finitos.

- Desde el punto de vista del control de calidad de las piezas es necesario desarrollar un software o metodología para predecir la aparición y magnitud del delaminado tipo II, en función de diferentes variables, como son el volumen de fibra, la orientación entre el tejido en las capas exteriores y la trayectoria de corte programada, la profundidad de pasada, etc. con el objetivo de plantear un mecanizado óptimo en cada tipo de pieza, y retrabajar únicamente las zonas donde esté previsto que haya delaminado.

- Analizar la aplicación de técnicas de optimización para encontrar las condiciones de mecanizado más adecuadas desde el punto de vista del delaminado, la rugosidad y el desgaste de la herramienta. 



\section{PUBLICACIONES}

La presente tesis ha dado lugar a las siguientes publicaciones y ponencias:

\section{ARTÍCULOS INDEXADOS}

M. D. Navarro-Mas, M. D. Meseguer, J. Lluch-Cerezo, and J. A. GarcíaManrique, "Comparison of different parameters to evaluate delamination in edge trimming of basalt fiber reinforced plastics (BFRP)," Materials (Basel)., vol. 13, no. 23, pp. 1-18, 2020, doi: 10.3390/ma13235326.

M. D. Navarro-Mas, J. A. García-Manrique, M. D. Meseguer, I. Ordeig, and A. I. Sánchez, "Delamination study in edge trimming of Basalt Fiber Reinforced Plastics (BFRP)," Materials (Basel)., vol. 11, no. 8, 2018, doi: 10.3390/ma11081418.

\section{ARTÍCULOS NO INDEXADOS}

M. D. Navarro-Mas, M. D. Meseguer, A. I. Sánchez, and S. C. Gutiérrez, "Tool wear study in edge trimming on basalt fibre reinforced plastics," Procedia Manuf., vol. 13, pp. 259-266, 2017, doi: 10.1016/j.promfg.2017.09.067. 


\section{PONENCIAS EN CONGRESOS}

M. D. Navarro-Mas, M. D. Meseguer, I. Ordeig and J. Lluch-Cerezo, "Milling angular references and process parameters on fiber reinforced plastics", Manufacturing Engineering Society International Conference, 2021, Gijón, Spain.

M. D. Navarro-Mas, M. D. Meseguer, and A. I. Sánchez, "Modelo de predicción de la rugosidad superficial en el mecanizado de un material compuesto con fibra de basalto," Creando Sinergias, 2016, Alcoy, Spain.

Esta Tesis Doctoral ha sido desarrollada en el marco de los proyectos de investigación:

2020-2023

PID2019-108807RB-I00: GEMELOS DIGITALES EN LOS PROCESOS DE FABRICACION ADITIVA PARA COMPONENTES DE FIBRA DE CARBONO: HACIA MOVILIDAD SOSTENIBLE.

Entidad Financiadora: Agencia Estatal de Investigación.

2014-2018

DPI2013-44903-R-AR: FABRICACION DE COMPOSITES DE ALTAS

PRESTACIONES SIN AUTOCLAVE (OOA).

Entidad Financiadora: Ministerio de Economía y Competitividad.

2011-2015

DPI2010-20333: DESARROLLO SOSTENIBLE Y MODELADO DE

COMPOSITES TERMOPLASTICOS (GREEN COMPOSITE).

Entidad Financiadora: Ministerio de Economía y Competitividad.
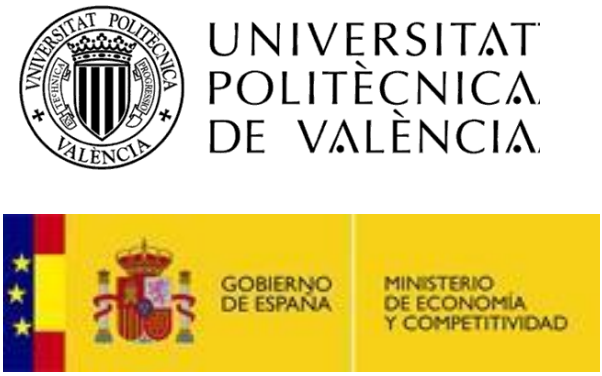


\section{BIBLIOGRAFÍA}

[1] S. Kalpakjian, "Manufacturing engineering and technology." Pearson Education, Upper Saddle River, 2014.

[2] W. Michaeli and M. Wegener, "Tecnología de los composites-plásticos reforzados." Hanser, Barcelona, 1992.

[3] A. Miravete, "Materiales compuestos. Tomos I y II." Zaragoza, 2000.

[4] M. M. A. Nassar, R. Arunachalam, and K. I. Alzebdeh, "Machinability of natural fiber reinforced composites: a review," Int. J. Adv. Manuf. Technol., vol. 88, no. 9-12, pp. 2985-3004, 2017, doi: 10.1007/s00170016-9010-9.

[5] D. K. Rajak, D. D. Pagar, P. L. Menezes, and E. Linul, "Fiber-reinforced polymer composites: Manufacturing, properties, and applications," Polymers (Basel)., vol. 11, no. 10, 2019, doi: 10.3390/polym11101667.

[6] A. K. Mohanty, M. Misra, and G. Hinrichsen, "Biofibres, biodegradable polymers and biocomposites: An overview," Macromol. Mater. Eng., vol. 276-277, pp. 1-24, 2000, doi: 10.1002/(SICI)14392054(20000301)276:1<1::AID-MAME1>3.0.CO;2-W.

[7] V. Lopresto, C. Leone, and I. De Iorio, "Mechanical characterisation of basalt fibre reinforced plastic," Compos. Part B Eng., vol. 42, no. 4, pp. 717-723, 2011, doi: 10.1016/j.compositesb.2011.01.030. 
[8] Sandvik Coromant, "Sandvik Coromant C-2920:30 User's guide: composite solutions. Machining carbon fibre materials." www.sandvik.coromant.com.

[9] V. Dhand, G. Mittal, K. Y. Rhee, S. J. Park, and D. Hui, "A short review on basalt fiber reinforced polymer composites," Compos. Part B Eng., vol. 73, pp. 166-180, 2015, doi: 10.1016/j.compositesb.2014.12.011.

[10] V. Manikandan, J. T. Winowlin Jappes, S. M. Suresh Kumar, and P. Amuthakkannan, "Investigation of the effect of surface modifications on the mechanical properties of basalt fibre reinforced polymer composites," Compos. Part B Eng., vol. 43, no. 2, pp. 812-818, 2012, doi: 10.1016/j.compositesb.2011.11.009.

[11] Z. Lu, G. Xian, and H. Li, "Effects of elevated temperatures on the mechanical properties of basalt fibers and BFRP plates," Constr. Build. Mater., vol. 127, pp. 1029-1036, 2016, doi: 10.1016/j.conbuildmat.2015.10.207.

[12] G. Vikas and M. Sudheer, "A review on properties of basalt fiber reinforced polymer composites," Am. J. Mater. Sci., vol. 7, no. 5, pp. 156165, 2017, doi: 10.5923/j.materials.20170705.07.

[13] T. Scalici, G. Pitarresi, D. Badagliacco, V. Fiore, and A. Valenza, "Mechanical properties of basalt fiber reinforced composites manufactured with different vacuum assisted impregnation techniques," Compos. Part B Eng., vol. 104, pp. 35-43, 2016, doi: 10.1016/j.compositesb.2016.08.021.

[14] T. Bhat, D. Fortomaris, E. Kandare, and A. P. Mouritz, "Properties of thermally recycled basalt fibres and basalt fibre composites," J. Mater. Sci., vol. 53, no. 3, pp. 1933-1944, 2018, doi: 10.1007/s10853-017-16727.

[15] I. M. De Rosa et al., "Post-impact mechanical characterisation of glass and basalt woven fabric laminates," Appl. Compos. Mater., vol. 19, no. 3-4, pp. 475-490, 2012, doi: 10.1007/s10443-011-9209-8.

[16] V. Acar, F. Cakir, E. Alyamaç, and M. Ö. Seydibeyoğlu, "Basalt fibers," in Fiber Technology for Fiber-Reinforced Composites, Elsevier Ltd, 2017, pp. 169-185.

[17] V. Fiore, T. Scalici, G. Di Bella, and A. Valenza, "A review on basalt fibre and its composites," Compos. Part B Eng., vol. 74, pp. 74-94, 2015, doi: 10.1016/j.compositesb.2014.12.034.

[18] V. Fiore, G. Di Bella, and A. Valenza, "Glass-basalt/epoxy hybrid composites for marine applications," Mater. Des., vol. 32, no. 4, pp. 20912099, 2011, doi: 10.1016/j.matdes.2010.11.043. 
[19] T. Roberts, The Carbon Fibre Industry Worldwide 2011-2020: An Evaluation of Current Markets and Future Supply and Demand. 2011.

[20] R. Domingo, B. de Agustina, M. M. Marin, and R. Calvo, "Latest trends in basalt fibre applications and implications of machining processes," 2021.

[21] D. K. Rajak, D. D. Pagar, R. Kumar, and C. I. Pruncu, "Recent progress of reinforcement materials: A comprehensive overview of composite materials," J. Mater. Res. Technol., vol. 8, no. 6, pp. 6354-6374, 2019, doi: 10.1016/j.jmrt.2019.09.068.

[22] M. H. Hassan, A. R. Othman, and S. Kamaruddin, "A review on the manufacturing defects of complex-shaped laminate in aircraft composite structures," Int. J. Adv. Manuf. Technol., vol. 91, no. 9-12, pp. 4081-4094, 2017, doi: 10.1007/s00170-017-0096-5.

[23] J. A. García Manrique, J. D. Zamanillo Cantolla, and F. J. Chinesta Soria, "Gestión del frente de avance en la modelización numérica del conformado por transferencia de resina (RTM)." Universidad Politécnica de Valencia, Valencia, 2000.

[24] J. Fleischer, R. Teti, G. Lanza, P. Mativenga, H. C. Möhring, and A. Caggiano, "Composite materials parts manufacturing," CIRP Ann., vol. 67, no. 2, pp. 603-626, 2018, doi: 10.1016/j.cirp.2018.05.005.

[25] J. Figueras, "El composite como material a mecanizar. IK4-Ideko."

[26] V. Lopresto, A. Caggiano, and R. Teti, "High Performance Cutting of Fibre Reinforced Plastic Composite Materials," Procedia CIRP, vol. 46, pp. 71-82, 2016, doi: 10.1016/j.procir.2016.05.079.

[27] A. Caggiano, "Machining of fibre reinforced plastic composite materials," Materials (Basel)., vol. 11, no. 3, 2018, doi: 10.3390/ma11030442.

[28] J. Sheikh-Ahmad, Machining of Polymer Composites, 1st ed. 20. 2009.

[29] J. Sheikh-Ahmad, N. Urban, and H. Cheraghi, "Machining damage in edge trimming of CFRP," Mater. Manuf. Process., vol. 27, no. 7, pp. 802-808, 2012, doi: 10.1080/10426914.2011.648253.

[30] J. P. Davim, Machining composite materials. Hoboken, New Jersey: Wiley, 2010.

[31] T. Chen, J. Xiang, F. Gao, X. Liu, and G. Liu, "Study on cutting performance of diamond-coated rhombic milling cutter in machining carbon fiber composites," Int. J. Adv. Manuf. Technol., vol. 103, no. 9-12, pp. 4731-4737, 2019, doi: 10.1007/s00170-019-03902-z.

[32] Sandvik Coromant, "Sandvik Coromant C1040-184 Fresas optimizadas 
para composites." www.sandvik.coromant.com.

[33] Mitsubishi, "Mitsubishi P715G Solutions for composites." www.mitsubishicarbide.com.

[34] G. T. Smith, Cutting Tool Technology: Industrial Handbook, 1st ed. 20. 2008.

[35] S. Kumar, G. Meenu, and P. S. Satsangi, "A genetic algorithmic approach for optimization of surface roughness prediction model in turning using UD-GFRP composite," Indian J. Eng. Mater. Sci., vol. 19, no. 6, pp. 386396, 2012.

[36] K. Palanikumar, L. Karunamoorthy, and R. Karthikeyan, "Assessment of factors influencing surface roughness on the machining of glass fiberreinforced polymer composites," Mater. Des., vol. 27, no. 10, pp. 862871, 2006, doi: 10.1016/j.matdes.2005.03.011.

[37] J. R. Ferreira, N. L. Coppini, and G. W. A. Miranda, "Machining optimisation in carbon fibre reinforced composite materials," J. Mater. Process. Technol., vol. 92-93, pp. 135-140, 1999, doi: 10.1016/S09240136(99)00221-6.

[38] J. Campos Rubio, A. M. Abrao, P. E. Faria, A. E. Correia, and J. P. Davim, "Effects of high speed in the drilling of glass fibre reinforced plastic: Evaluation of the delamination factor," Int. J. Mach. Tools Manuf., vol. 48, no. 6, pp. 715-720, 2008, doi: 10.1016/j.ijmachtools.2007.10.015.

[39] J. P. Davim, J. C. Rubio, and A. M. Abrao, "A novel approach based on digital image analysis to evaluate the delamination factor after drilling composite laminates," Compos. Sci. Technol., vol. 67, no. 9, pp. 19391945, 2007, doi: 10.1016/j.compscitech.2006.10.009.

[40] J. P. Davim and P. Reis, "Drilling carbon fiber reinforced plastics manufactured by autoclave-experimental and statistical study," Mater. Des., vol. 24, no. 5, pp. 315-324, 2003, doi: 10.1016/S02613069(03)00062-1.

[41] A. Faraz, D. Biermann, and K. Weinert, "Cutting edge rounding: An innovative tool wear criterion in drilling CFRP composite laminates," Int. J. Mach. Tools Manuf., vol. 49, no. 15, pp. 1185-1196, 2009, doi: 10.1016/j.ijmachtools.2009.08.002.

[42] N. Feito, A. S. Milani, and A. Muñoz-Sánchez, "Drilling optimization of woven CFRP laminates under different tool wear conditions: a multiobjective design of experiments approach," Struct. Multidiscip. Optim., vol. 53, no. 2, pp. 239-251, 2016, doi: 10.1007/s00158-015-1324-y.

[43] V. N. Gaitonde, S. R. Karnik, J. C. Rubio, A. E. Correia, A. M. Abrão, and 
BIBLIOGRAFÍA

J. P. Davim, "Analysis of parametric influence on delamination in highspeed drilling of carbon fiber reinforced plastic composites," J. Mater. Process. Technol., vol. 203, no. 1-3, pp. 431-438, 2008, doi: 10.1016/j.jmatprotec.2007.10.050.

[44] S. Gaugel et al., "A comparative study on tool wear and laminate damage in drilling of carbon-fiber reinforced polymers (CFRP)," Compos. Struct., vol. 155, pp. 173-183, 2016, doi: 10.1016/j.compstruct.2016.08.004.

[45] T. J. Grilo, R. M. F. Paulo, C. R. M. Silva, and J. P. Davim, "Experimental delamination analyses of CFRPs using different drill geometries," Compos. Part B Eng., vol. 45, no. 1, pp. 1344-1350, 2013, doi: 10.1016/j.compositesb.2012.07.057.

[46] V. Krishnaraj et al., "Optimization of machining parameters at high speed drilling of carbon fiber reinforced plastic (CFRP) laminates," Compos. Part B Eng., vol. 43, no. 4, pp. 1791-1799, 2012, doi: 10.1016/j.compositesb.2012.01.007.

[47] A. I. Azmi, R. J. T. Lin, and D. Bhattacharyya, "Machinability study of glass fibre-reinforced polymer composites during end milling," Int. J. Adv. Manuf. Technol., vol. 64, no. 1-4, pp. 247-261, 2013, doi: 10.1007/s00170-012-4006-6.

[48] J. P. Davim and P. Reis, "Damage and dimensional precision on milling carbon fiber-reinforced plastics using design experiments," J. Mater. Process. Technol., vol. 160, no. 2, pp. 160-167, 2005, doi: 10.1016/j.jmatprotec.2004.06.003.

[49] Y. He, H. Qing, S. Zhang, D. Wang, and S. Zhu, "The cutting force and defect analysis in milling of carbon fiber-reinforced polymer (CFRP) composite," Int. J. Adv. Manuf. Technol., vol. 93, no. 5-8, pp. 1829-1842, 2017, doi: 10.1007/s00170-017-0613-6.

[50] M. P. Jenarthanan and R. Jeyapaul, "Optimisation of machining parameters on milling of GFRP composites by desirability function analysis using Taguchi method," Int. J. Eng. Sci. Technol., vol. 5, no. 4, p. 22, 2013, doi: 10.4314/ijest.v5i4.3.

[51] E. Kiliçkap, A. Yardimeden, and Y. H. Çelik, "Investigation of experimental study of end milling of CFRP composite," Sci. Eng. Compos. Mater., vol. 22, no. 1, pp. 89-95, 2015, doi: 10.1515/secm-2013-0143.

[52] N. Li, J. Y. Sheikh-Ahmad, A. El-Sinawi, and V. Krishnaraj, "Multiobjective optimization of the trimming operation of CFRPs using sensorfused neural networks and TOPSIS," Meas. J. Int. Meas. Confed., vol. 132, pp. 252-262, 2019, doi: 10.1016/j.measurement.2018.09.057.

[53] P. Masek, P. Zeman, and P. Kolar, "Edge trimming of C / PPS plates," Int. 
J. Adv. Manuf. Technol., vol. 101, pp. 157-170, 2019.

[54] P. Patel, V. Chaudhary, K. Patel, and P. Gohil, "Milling of Polymer Matrix Composites : A Review," vol. 13, no. 10, pp. 7455-7465, 2018.

[55] M. Haddad, R. Zitoune, H. Bougherara, F. Eyma, and B. Castanié, "Study of trimming damages of CFRP structures in function of the machining processes and their impact on the mechanical behavior," Compos. Part B Eng., vol. 57, pp. 136-143, 2014, doi: 10.1016/j.compositesb.2013.09.051.

[56] M. Li, M. Huang, X. Jiang, C. liang Kuo, and X. Yang, "Study on burr occurrence and surface integrity during slot milling of multidirectional and plain woven CFRPs," Int. J. Adv. Manuf. Technol., vol. 97, no. 1-4, pp. 163-173, 2018, doi: 10.1007/s00170-018-1937-6.

[57] Ö. Erkan, B. Iş1k, A. Çiçek, and F. Kara, "Prediction of damage factor in end milling of glass fibre reinforced plastic composites using artificial neural network," Appl. Compos. Mater., vol. 20, no. 4, pp. 517-536, 2013, doi: 10.1007/s10443-012-9286-3.

[58] H. Vasudevan, R. R. Rajguru, and N. C. Deshpande, "Experimental Investigation and Optimization in Edge Milling of NEMA G-11 GFRP/Epoxy Composites," Procedia Mater. Sci., vol. 5, pp. 2105-2114, 2014, doi: 10.1016/j.mspro.2014.07.546.

[59] M. Haddad, R. Zitoune, F. Eyma, and B. Castanie, "Study of the surface defects and dust generated during trimming of CFRP: Influence of tool geometry, machining parameters and cutting speed range," Compos. Part A Appl. Sci. Manuf., vol. 66, pp. 142-154, 2014, doi: 10.1016/j.compositesa.2014.07.005.

[60] D. Ozkan, P. Panjan, M. S. Gok, and A. C. Karaoglanli, "Experimental study on toolwear and delamination in milling CFRPs with TiAlN-and TiN-coated tools," Coatings, vol. 10, no. 7, 2020, doi: 10.3390/coatings 10070623 .

[61] P. Praveen Raj and A. Elaya Perumal, "Taguchi analysis of surface roughness and delamination associated with various cemented carbide K10 end mills in milling of GFRP," J. Eng. Sci. Technol. Rev., vol. 3, no. 1, pp. 58-64, 2010, doi: 10.25103/jestr.031.11.

[62] M. R. Razfar and M. R. Z. Zadeh, "Optimum damage and surface roughness prediction in end milling glass fibre-reinforced plastics, using neural network and genetic algorithm," Proc. Inst. Mech. Eng. Part B J. Eng. Manuf., vol. 223, no. 6, pp. 653-664, 2009, doi: 10.1243/09544054JEM1409.

[63] M. P. Jenarthanan, R. Jeyapaul, and N. Neeli, "Analysis of factors influencing delamination in milling process of glass fibre reinforced 
BIBLIOGRAFÍA

plastic (GFRP) composite materials," Multidiscip. Model. Mater. Struct., vol. 9, no. 3, pp. 293-306, 2013, doi: 10.1108/MMMS-03-2012-0005.

[64] R. Sreenivasulu, "Optimization of surface roughness and delamination damage of GFRP composite material in end milling using taguchi design method and artificial neural network," Procedia Eng., vol. 64, pp. 785794, 2013, doi: 10.1016/j.proeng.2013.09.154.

[65] M. Slamani, J. F. Chatelain, and H. Hamedanianpour, "Influence of machining parameters on surface quality during high speed edge trimming of carbon fiber reinforced polymers," Int. J. Mater. Form., vol. 12, no. 3, pp. 331-353, 2019, doi: 10.1007/s12289-018-1419-2.

[66] X. Rimpault, J. F. Chatelain, J. E. Klemberg-Sapieha, and M. Balazinski, "Tool wear and surface quality assessment of CFRP trimming using fractal analyses of the cutting force signals," CIRP J. Manuf. Sci. Technol., vol. 16, pp. 72-80, 2017, doi: 10.1016/j.cirpj.2016.06.003.

[67] R. Voss, L. Seeholzer, F. Kuster, and K. Wegener, "Influence of fibre orientation, tool geometry and process parameters on surface quality in milling of CFRP," CIRP J. Manuf. Sci. Technol., vol. 18, pp. 75-91, 2017, doi: 10.1016/j.cirpj.2016.10.002.

[68] G. Liu, H. Chen, Z. Huang, F. Gao, and T. Chen, "Surface quality of staggered PCD end mill in milling of carbon fiber reinforced plastics," Appl. Sci., vol. 7, no. 2, 2017, doi: 10.3390/app7020199.

[69] F. Robbany, B. Pramujati, Suhardjono, M. K. Effendi, B. O. P. Soepangkat, and R. Norcahyo, "Multi response prediction of cutting force and delamination in carbon fiber reinforced polymer using backpropagation neural network-genetic algorithm," AIP Conf. Proc., vol. 2114, 2019, doi: 10.1063/1.5112416.

[70] F. Wang, J. Yin, J. Ma, Z. Jia, F. Yang, and B. Niu, "Effects of cutting edge radius and fiber cutting angle on the cutting-induced surface damage in machining of unidirectional CFRP composite laminates," Int. J. Adv. Manuf. Technol., vol. 91, no. 9-12, pp. 3107-3120, 2017, doi: 10.1007/s00170-017-0023-9.

[71] D. Wang, F. Wang, Z. Wang, G. Bi, and Q. Wang, Influence of Dynamic Change of Fiber Cutting Angle on Surface Damage in CFRP Milling, vol. 923. Springer Singapore, 2018.

[72] W. Hintze Wolfgang, D. Hartmann, and C. Schütte, "Occurrence and propagation of delamination during the machining of carbon fibre reinforced plastics (CFRPs) - An experimental study," Compos. Sci. Technol., vol. 71, no. 15, pp. 1719-1726, 2011, doi: 10.1016/j.compscitech.2011.08.002. 
[73] S. A. Sundi, R. Izamshah, and M. S. Kasim, "Effect of machining parameters on surface roughness in edge trimming of carbon fiber reinforced plastics (CFRP)," Tribol. Online, vol. 15, no. 2, pp. 53-59, 2020, doi: 10.2474/TROL.15.53.

[74] J. Sheikh-Ahmad, M. Dhuttargaon, and H. Cheraghi, "New tool life criterion for delamination free milling of CFRP," Int. J. Adv. Manuf. Technol., vol. 92, no. 5-8, pp. 2131-2143, 2017, doi: 10.1007/s00170017-0240-2.

[75] W. Hintze, M. Cordes, and G. Koerkel, "Influence of weave structure on delamination when milling CFRP," J. Mater. Process. Technol., vol. 216, pp. 199-205, 2015, doi: 10.1016/j.jmatprotec.2014.09.004.

[76] N. Neeli, M. P. Jenarthanan, and G. Dileep Kumar, "Multi-response optimization for machining GFRP composites using GRA and DFA," Multidiscip. Model. Mater. Struct., vol. 14, no. 3, pp. 482-496, 2018, doi: 10.1108/MMMS-08-2017-0092.

[77] M. D. Navarro-Mas, J. A. García-Manrique, M. D. Meseguer, I. Ordeig, and A. I. Sánchez, "Delamination study in edge trimming of Basalt Fiber Reinforced Plastics (BFRP)," Materials (Basel)., vol. 11, no. 8, 2018, doi: 10.3390/ma11081418.

[78] M. D. Navarro-Mas, M. D. Meseguer, J. Lluch-Cerezo, and J. A. GarcíaManrique, "Comparison of different parameters to evaluate delamination in edge trimming of basalt fiber reinforced plastics (BFRP)," Materials (Basel)., vol. 13, no. 23, pp. 1-18, 2020, doi: 10.3390/ma13235326.

[79] T. Geis, C. Klingelhöller, and W. Hintze, "Constant depth scoring of fibre reinforced plastic structures to prevent delamination," Procedia CIRP, vol. 14, pp. 205-210, 2014, doi: 10.1016/j.procir.2014.03.103.

[80] F. Islam, J. Ramkumar, and A. S. Milani, "A simplified damage prediction framework for milling of unidirectional carbon fiber-reinforced plastics," Adv. Manuf. Polym. Compos. Sci., vol. 1, no. 4, pp. 175-184, 2015, doi: 10.1080/20550340.2015.1117747.

[81] F. Wang, B. Zhang, Z. Jia, X. Zhao, and Q. Wang, "Structural optimization method of multitooth cutter for surface damages suppression in edge trimming of Carbon Fiber Reinforced Plastics," J. Manuf. Process., vol. 46, no. August, pp. 204-213, 2019, doi: 10.1016/j.jmapro.2019.09.013.

[82] D. Nguyen, M. S. Bin Abdullah, R. Khawarizmi, D. Kim, and P. Kwon, "The effect of fiber orientation on tool wear in edge-trimming of carbon fiber reinforced plastics (CFRP) laminates," Wear, vol. 450-451, no. February, p. 203213, 2020, doi: 10.1016/j.wear.2020.203213.

[83] A. Gilpin, "Tool solutions for machining composites," Reinf. Plast., vol. 
BIBLIOGRAFÍA

53, no. 6, pp. 30-33, 2009, doi: 10.1016/S0034-3617(09)70260-7.

[84] M. Slamani, J. F. Chatelain, and H. Hamedanianpour, "Comparison of two models for predicting tool wear and cutting force components during high speed trimming of CFRP," Int. J. Mater. Form., vol. 8, no. 2, pp. 305-316, 2015, doi: 10.1007/s12289-014-1170-2.

[85] A. I. Azmi, "Monitoring of tool wear using measured machining forces and neuro-fuzzy modelling approaches during machining of GFRP composites," Adv. Eng. Softw., vol. 82, pp. 53-64, 2015, doi: 10.1016/j.advengsoft.2014.12.010.

[86] ISO, "ISO 8688-1989, Tool-life testing in milling. Part 1: End milling.".

[87] M. Kim, M. Lee, G. Cho, and S. K. Lee, "Effect of the Fiber Orientation and the Radial Depth of Cut on the Flank Wear in End Milling of CFRP," Int. J. Precis. Eng. Manuf., vol. 21, no. 7, pp. 1187-1199, 2020, doi: 10.1007/s12541-020-00340-w.

[88] W. Hintze and F. Brügmann, "Influence of Curved Workpiece Contours on Delamination during end Milling of FRP," Procedia CIRP, vol. 62, pp. 62-67, 2017, doi: 10.1016/j.procir.2016.06.115.

[89] K. Colligan and M. Ramulu, "Delamination in surface plies of graphitelepoxy caused by the edge trimming process," Process. Manuf. Compos. Mater., vol. 27, no. JANUARY 1991, pp. 113-125, 1991.

[90] K. Szwajka and T. Trzepieciński, "Effect of tool material on tool wear and delamination during machining of particleboard," J. Wood Sci., vol. 62, no. 4, pp. 305-315, 2016, doi: 10.1007/s10086-016-1555-6.

[91] F. Wang, J. Yin, Z. Jia, B. Niu, and W. Liu, "A novel approach to evaluate the delamination extent after edge trimming of carbon-fiber-reinforced composites," Proc. Inst. Mech. Eng. Part B J. Eng. Manuf., vol. 232, no. 14, pp. 2523-2532, 2018, doi: 10.1177/0954405417699015.

[92] Y. He, J. Sheikh-Ahmad, S. Zhu, and C. Zhao, "Cutting force analysis considering edge effects in the milling of carbon fiber reinforced polymer composite," J. Mater. Process. Technol., vol. 279, no. October 2019, p. 116541, 2020, doi: 10.1016/j.jmatprotec.2019.116541.

[93] W. Hintze and D. Hartmann, "Modeling of delamination during milling of unidirectional CFRP," Procedia CIRP, vol. 8, pp. 444-449, 2013, doi: 10.1016/j.procir.2013.06.131.

[94] W. Hintze and F. Brügmann, "Influence of spatial tool inclination on delamination when milling CFRP," J. Mater. Process. Technol., vol. 252, no. June 2017, pp. 830-837, 2018, doi: 10.1016/j.jmatprotec.2017.10.041.

[95] Mitsubishi, "Mitsubishi catálogo general herramientas de metal duro. 
C006S 2014." www.mitsubishicarbide.com.

[96] Mitsubishi, "Mitsubishi B116S AXD Fresa multifuncional para el mecanizado de aleaciones de alumnio y titanio.".

[97] S. Johansen, "The Welch-James approximation to the distribution of the residual sum of squares in a weighted linear regression," Biometrika, vol. 67, no. 1, pp. 85-92, 1980, doi: 10.1093/biomet/67.1.85.

[98] P. Mair and R. Wilcox, "Robust statistical methods in R using the WRS2 package," Behav. Res. Methods, vol. 52, no. 2, pp. 464-488, 2020, doi: 10.3758/s13428-019-01246-w.

[99] J. W. Mauchly, "Significance Test for Sphericity of a Normal n-Variate Distribution," Ann. Math. Stat., vol. 11, no. 2, pp. 204-209, 1940, doi: 10.1214/aoms/1177731915.

[100] A. Pardo and R. San Martín, Análisis de datos en ciencias sociales y de la salud II. Madrid: Síntesis, 2010.

[101] R. Koenker and G. Bassett, "Regression Quantiles," Econometrica, vol. 46, no. 1, pp. 33-50, 1978, doi: 10.2307/1913643.

[102] M. D. Navarro-Mas, M. D. Meseguer, and A. I. Sánchez, "Modelo de predicción de la rugosidad superficial en el mecanizado de un material compuesto con fibra de basalto .," IV Congreso I+D+I Campus de Alcoi. CREANDO SINERGIAS, 2016.

[103] M. D. Navarro-Mas, M. D. Meseguer, A. I. Sánchez, and S. C. Gutiérrez, "Tool wear study in edge trimming on basalt fibre reinforced plastics," Procedia Manuf., vol. 13, pp. 259-266, 2017, doi: 10.1016/j.promfg.2017.09.067.

[104] C. Wang, G. Liu, Q. An, and M. Chen, "Occurrence and formation mechanism of surface cavity defects during orthogonal milling of CFRP laminates," Compos. Part B Eng., vol. 109, pp. 10-22, 2017, doi: 10.1016/j.compositesb.2016.10.015.

[105] M. D. Navarro-Mas, M. D. Meseguer, I. Ordeig, and J. Lluch-Cerezo, "Milling angular references and process parameters on fiber reinforced plastics," in Manufacturing engineering society international conference, 2021. 


\section{ANEXOS}

\section{ANEXOS AL CAPÍTULO 2}

\section{I.1. CARACTERÍSTICAS DE LA RESINA}

Mixing and Handling

PRIMETM 20LV resin must be mixed with PRIMETM 20 hardener in the following ratio:

PRIME $^{\text {TM }}$ 20LV resin: Prime 20 hardener (fast, Slow or Extra Slow)

\section{$100: 26$ (by weight)}

$100: 31.4$ (by volume)

The fast hardener is not usually used alone with the resin although it can be used in this way, it is more often premixed with another PRIMETM 20 hardener to achieve shorter gel times than would otherwise be obtained with the use of Slow or Extra Slow hardener alone. The premixed hardener combination (Fast + Slow, or Fast + Extra Slow) is still mixed with resin at $100: 26$ by weight.

Accurate measurement and thorough mixing are essential when using this system, and any deviation from the prescribed mixing ratios will seriously degrade the physical properties of the cured system. The resin and hardener must be well stirred for two minutes or more, with particular attention being paid to the sides and bottom of the container. As soon as the material is mixed the reaction begins. This reaction produces heat (exothermic), which will in turn accelerate the reaction. If this mixed material is left in a confined mixing vessel the heat cannot disperse, and the reaction will become uncontrollable. See "Working Properties" for details

\section{Application}

PRIMETM 20LV system is intended for use in any established resin infusion process. The information provided in the tables in this datasheet should allow the user to achieve a successful result with PRIMETM 20LV system. However, if further information is required, please contact Technical Services.

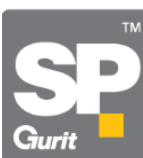

Cure Schedule

To generate optimum mechanical properties for this system an elevated temperature cure is required. The recommended minimum cure schedule is 7 hours at $65^{\circ} \mathrm{C}$ or 16 hours at $50^{\circ} \mathrm{C}$. Ambient temperature $\left(15-25^{\circ} \mathrm{C}\right)$ cure of this system will not generate adequate properties and is therefore not recommended.

Parts can be 'pre-cured' in the mould at temperatures just above ambient (e.g. $35-45^{\circ} \mathrm{C}$ ) to give the part sufficient strength and stiffness to allow earlier demoulding. Such parts should still be post cured at the minimum recommended time/temperature indicated above. Contact Technical Services for 'pre-cure' time/temperature recommendations.

\begin{tabular}{|l|c|c|c|c|}
\hline \multirow{2}{*}{ Table 2. Working Properties } \\
\hline & \multicolumn{4}{|c|}{ Fast Hardener } \\
\cline { 2 - 5 } & $15^{\circ} \mathrm{C}$ & $\mathbf{2 0 ^ { \circ } \mathrm { C }}$ & $\mathbf{2 5 ^ { \circ } \mathrm { C }}$ & $\mathbf{3 0 ^ { \circ } \mathrm { C }}$ \\
\hline $\begin{array}{l}\text { Initial Mixed } \\
\text { Viscosity (cP) }\end{array}$ & $535-568$ & $318-338$ & $219-232$ & $127-134$ \\
\hline $\begin{array}{l}\text { Geltime - Tecam } \\
\text { 155g in water } \\
\text { (hr }: \text { min) }\end{array}$ & $1: 30$ & $1: 09$ & $0: 30$ & $0: 17$ \\
\hline $\begin{array}{l}\text { Pot life 500g in air (hr } \\
\text { : min) }\end{array}$ & $0: 35$ & $0: 28$ & $0: 23$ & $0: 16$ \\
\hline $\begin{array}{l}\text { Latest flow under } \\
\text { vacuum (theoretical, } \\
\text { thin film, hr : min) }\end{array}$ & $3: 50$ & $3: 10$ & $2: 40$ & $1: 50$ \\
\hline $\begin{array}{l}\text { Earliest vacuum off } \\
\text { time (theoretical thin } \\
\text { film) (thr : min) }\end{array}$ & $5: 10$ & $4: 15$ & $3: 20$ & $2: 15$ \\
\hline $\begin{array}{l}\text { Demould time } \\
\text { (hr : min) }\end{array}$ & $9: 00$ & $6: 45$ & $5: 00$ & $2: 30$ \\
\hline
\end{tabular}

Notes: For an explanation of test methods used see 'Formulated Products Technical Characteristics', which can be found in the 'Intro to Form Prods'. pdf. This can be found in the Formuled product section on the wesite muw guritcom All figures quoted are indicative of the properties of the product concerned. Some batch to batch variation may occur. - Demoulding components made with Slow or Extra Slow Hardener should only be carried out after the part has received an elevated temperature cure in the
mould.

\section{Transport \& Storage}

The system should be kept in securely closed containers during transport and storage.

Storage should be in a dry place out of direct sunlight. The temperature should be between

$18^{\circ} \mathrm{C}$ and $25^{\circ} \mathrm{C}$. Containers should be firmly closed. The hardeners, in particular, will suffer serious degradation if left exposed to air. 


\section{I.2. CARACTERÍSTICAS DEL TEJIDO DE BASALTO}

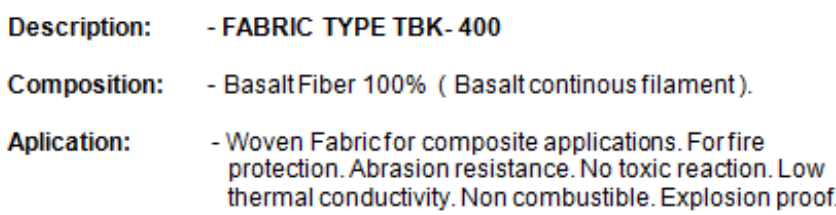

TYPICAL PROPERTIES:

\begin{tabular}{|c|c|c|c|c|}
\hline PROPERTY & TEST METHOD & NOMINAL VALUE & TOLERANCE & UNIT \\
\hline THICKNESS & ISO $9073-2$ & 0,40 & $+/-3 \%$ & $\mathrm{~mm}$ \\
\hline MASS PER UNIT AREA & ISO 9073-1 & 400 & $+/-10 \%$ & $\mathrm{~g} / \mathrm{m}^{2}$ \\
\hline TENSILE STRENGTH & 1504606 & $\begin{array}{c}\text { Warp (urdimbre) } \\
>3050 \\
\text { Weft (trams) } \\
>2125\end{array}$ & $>$ & $\mathrm{N} / 25 \mathrm{~mm}$ \\
\hline COMBUSTIBILITY & NF P92-503 & - & - & MO \\
\hline $\begin{array}{l}\text { ABRASION CONTINUOS MAX. } \\
\text { TEMPERATURE }\end{array}$ & ISO12947-2 & $\begin{array}{c}-250+750^{\circ} \mathrm{C} \text { Fire } \\
\text { Blocking }\end{array}$ & & ${ }^{\circ} \mathrm{C}$ \\
\hline MELTING POINT & & $900^{\circ} \mathrm{C}$ & +-100 & ${ }^{\circ} \mathrm{C}$ \\
\hline $\begin{array}{l}\text { MOISTURE CONTENT OF BASALTIC } \\
\text { ROCK }\end{array}$ & & 0,1 & $+-0,05$ & $\%$ \\
\hline REACTION TO FIRE & FD P 92-507 & Pass & $+-0,8$ & . \\
\hline RESISTANCE TO MATCH FLAME & BS EN 597-2 & Pass & & \\
\hline $\begin{array}{l}\text { RESISTANCE TO SMOULDERING } \\
\text { CIGARRETE }\end{array}$ & BS EN 597-1 & Pass & & \\
\hline
\end{tabular}

\section{Roll and spool dimensions:}

\begin{tabular}{|c|c|}
\hline Slit width $(\mathrm{mm})$ & 1.000 \\
\hline Max. OD $(\mathrm{mm})$ & 240 \\
\hline Length $(\mathrm{m})$ & 80 \\
\hline ID $(\mathrm{mm})$ & 110 \\
\hline
\end{tabular}


PRODUCTS:

Products based on basalt continous filament including; basalt roving, basalt chopped strands, basalt fabrics, basalt mesh, basalt mats and felts.

\section{INGREDIENTS:}

Basalt continous filament $>99 \%$

Surface Binder/Sizing $<0,6 \%$

Chemical composition of Basalt continous Filament.

$\mathrm{SiO}_{2} \approx 50 \%$ Wt., $\mathrm{CaO}$. $\mathrm{Na}_{2} \mathrm{O}, \mathrm{FeO}, \mathrm{Al}_{2} \mathrm{O}_{3}$

Chemical Composition of surface binder/sizing.

All used surface binder/sizing are not harzardous and based on silane

PHYSICAL DATA:

Material (at normal condition)

Solid.

FIRE AND EXPLOSIVE:

Special fire fighting procedures

Non flammable.

REACTIVITY DATA:

Stability

Stable.

Hazardous decomposition products

None

TOXICOLOGICAL INFORMATION

\section{Effect of overexposure}

Acute-Basalt continuous filaments may cause mechanical irritation to the skin, eye and upper respiratory tract.

Chronic- There is no known health effects connected with long term use or contact with this product.

Precations: Avoid contact with eyes. Avoid contact with skin. Avoid breathing dust. Do not swallow. Do not eat, drink or smoke in work area. Wash thoroughly after handling

\section{EMERGENCY AND FIRST AID PROCEDURES}

Inhalation: Move person to fresh air. Seek medical attention if irritation persists Eye contact: Flush eyes with running water for at least 15 minutes. Seek medical attention if irritation persists.

Skin contact: Wash with mild soap and running water. Use a washcloth to help remove fibers. To avoid more irritation, do not rub or scratch effected area. Seek medical attention if irritation persists.

Ingestion: Ingestion of this material is unlikely. If it does occur, watch the person for several days to make sure that intestinal blockage does not occur. 


\section{I.3. ESPECIFICACIONES CENTRO DE MECANIZADO. KONDIA B-500}

\begin{tabular}{|c|c|c|}
\hline MODELO & CARACTERISTICAS & B-500 \\
\hline \multirow{8}{*}{ MESA } & Superficie de trabajo & $700 \times 360 \mathrm{~mm}$ \\
\hline & Ranuras en $\mathrm{T}\left(\mathrm{N}^{\circ} \mathrm{x}\right.$ anchura $\mathrm{x}$ distancia) & $3 \times 18 \times 110 \mathrm{~mm}$ \\
\hline & Altura de la mesa al suelo & $840 \mathrm{~mm}$. \\
\hline & Máximo peso sobre la mesa & $300 \mathrm{~kg}$. \\
\hline & Curso longitudinal (Eje X) & $560 \mathrm{~mm}$. \\
\hline & Curso transversal (Eje $Y$ ) & $380 \mathrm{~mm}$. \\
\hline & Husillos a bolas $(X-Y)$ & $\varnothing 32 \times 10 \mathrm{~mm}$. de paso \\
\hline & Distancia del centro de la mesa a la columna & Mínimo 190 mm. / Máximo 570 mm. \\
\hline \multirow{7}{*}{ CABEZAL } & Cono del eje portafresas & ISO-40 \\
\hline & Curso vertical (Eje Z) & $380 \mathrm{~mm}$. \\
\hline & Gama de velocidades r.p.m. & 100 a 6000 / Opcional 150 a 10000 \\
\hline & Número de velocidades & infinitamente variable \\
\hline & Husillo a bolas (Z) & $\varnothing 32 \times 10 \mathrm{~mm}$. de paso \\
\hline & Distancia del eje a la mesa & Mínimo $120 \mathrm{~mm}$. / Máximo $500 \mathrm{~mm}$. \\
\hline & Distancia del eje a la columna & $390 \mathrm{~mm}$ \\
\hline \multirow{2}{*}{ AVANCES } & Velocidad de corte & s/Control Numérico \\
\hline & Velocidad de avance rápido & $15.000 \mathrm{~mm}$. minuto \\
\hline \multirow{3}{*}{ PRESTACIONES } & Precisión de posicionamiento & $\pm 0,01 \mathrm{~mm}$ \\
\hline & Repetibilidad & $\pm 0,005 \mathrm{~mm}$ \\
\hline & Resolución & $0,001 \mathrm{~mm}$. \\
\hline \multirow{7}{*}{ MOTORES } & Eje principal (cabezal). Corriente alterna & $3,7 / 5,5 \mathrm{Kw}$. \\
\hline & Eje $\mathrm{X}$ : Corriente alterna & $1 \mathrm{Kw}$. \\
\hline & Eje Y: Corriente alterna & $1 \mathrm{Kw}$. \\
\hline & Eje Z: Corriente alterna & $1,2 \mathrm{Kw}$. \\
\hline & Bomba de refrigeración & $0,35 \mathrm{Kw}$ \\
\hline & Bomba de engrase cabezal & $0,25 \mathrm{Kw}$. \\
\hline & Enfriador de aceite & $0,25 \mathrm{Kw}$. \\
\hline \multirow{11}{*}{$\begin{array}{c}\text { CAMBIADOR } \\
\text { AUTOMATICO } \\
\text { DE } \\
\text { HERRAMIENTAS }\end{array}$} & Número de herramientas & 16 \\
\hline & Sistema de selección & secuencial \\
\hline & Máx. diámetro (herramientas contiguas) & $85 \mathrm{~mm}$. \\
\hline & Máx. diámetro (herramientas alternas) & $135 \mathrm{~mm}$. \\
\hline & Máx. longitud de la heramienta & $250 \mathrm{~mm}$. \\
\hline & Máx. peso admisible para 1 herramienta & $6 \mathrm{~kg}$. \\
\hline & Máx. peso para el total de herramientas & $60 \mathrm{~kg}$. \\
\hline & Portaherramientas & ISO-7388/1 Opcional BT-40 \\
\hline & Perno de tiro para fijación herramienta & ISO-7388/2-A Opcional BT-40-1 \\
\hline & Tiempo de cambio de herramienta & 6 segundos \\
\hline & Presión de aire & $5 / 7 \mathrm{~kg} . / \mathrm{cm}^{2}$ \\
\hline \multirow{4}{*}{ OTRAS } & Superficie en planta & $1800 \times 1950 \mathrm{~mm}$ \\
\hline & Altura de la máquina & $2200 \mathrm{~mm}$. \\
\hline & Peso de la máquina & $2800 \mathrm{~kg}$ \\
\hline & Potencia total instalada & $10 \mathrm{Kw}$ \\
\hline
\end{tabular}




\section{I.4. DATOS DE LA HERRAMIENTA}

\section{Selección de placas AXD4000}

Es necesario elegir la mejor placa en función del tipo de corte y del trabajo a realizar.

La $1^{\mathrm{a}}$ recomendación para corte estable es el rompevirutas $\mathrm{GL}$ con filo de corte resistente.

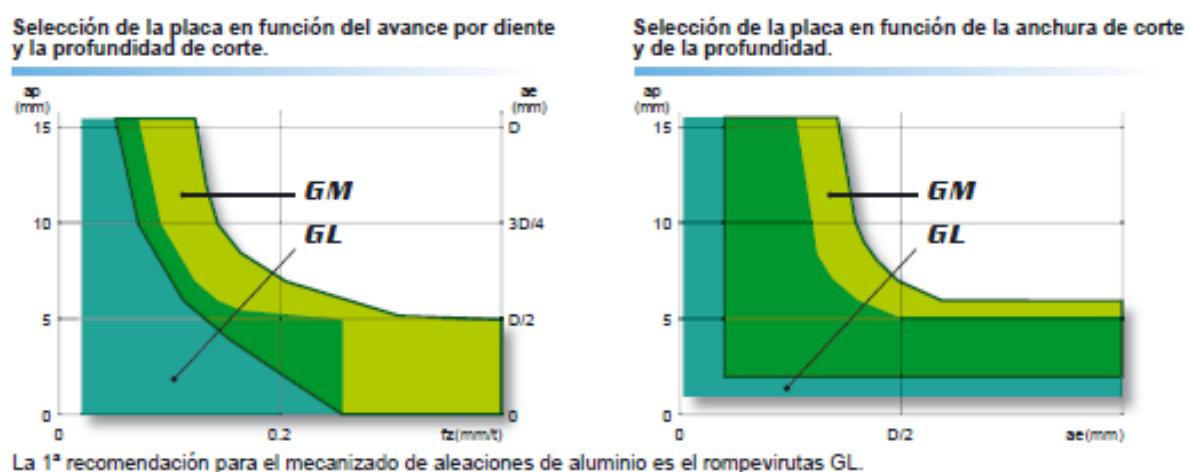

La $1^{2}$ recomendación para el mecanizado de aleaciones de aluminio es el rompevirutas GL

En casos de carga elevada como corte profundo o corte de avance elevado, se aconseja utilizar el rompevirutas GM.

Selección de la placa en función de la resistencia al desgaste
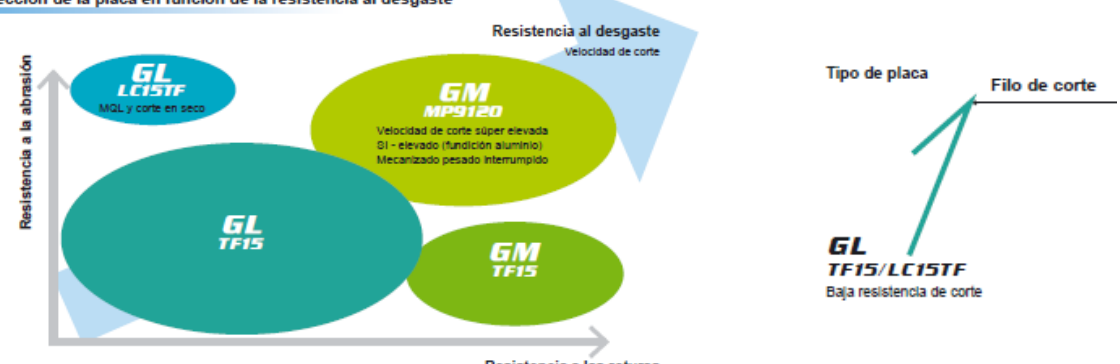

Resistencia a las roturas

Gala resistencla de corte

Portaherramientas

Número de referencia

AXD4000R252SA25SA
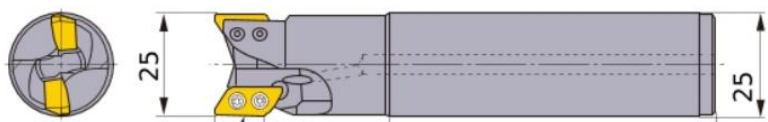

15.5

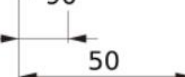

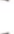

125

Plaquita
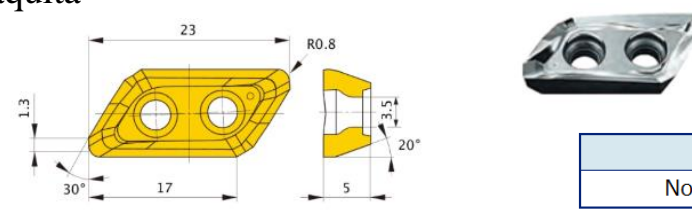

Número de referencia

XDGX175008PDFR-GL

Clasificación de calidades No recubierta $\cdot$ Metal Duro TF15 


\section{Guía operacional}

1) Utilice un chorro de aire o un cepillo para limpiar las bases de las placas antes de colocarlas.

2) Sujete las placas con firmeza contra la base y apriete los tornillos de fijación con la llave que se suministra.

3) Apriete los tornillos de fijación por orden, como se indica en la Figura 1.

4) Aplique lubricante en los tornillos de fijación y apriételos según el par especificado.

El par especificado es el siguiente:

AXD7000 $3,5 \mathrm{~N} \cdot \mathrm{m}(2,58 \mathrm{ft} \cdot \mathrm{lb})$

AXD4000 $1,5 \mathrm{~N} \cdot \mathrm{m}(1,11 \mathrm{ft} \cdot \mathrm{lb})$

5) Los tornillos de fijación son piezas importantes desde el punto de vista de la seguridad. Utilice la

referencia correcta de tornillos de fijación.

Si la velocidad del husillo es igual o superior a los valores de la Tabla 2, se recomienda sustituir los tornillos de fijación por unos nuevos cuando se cambien las placas.

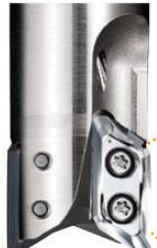

Fig.1

疍

\begin{tabular}{|c|c|c|}
\hline Tipo & \multicolumn{2}{|c|}{ AXn4000 } \\
\hline $\mathrm{D} 1(\mathrm{~mm})$ & ๑20 & ø25-ø125 \\
\hline Tornillo roscado & TS3SBS & TS3SB \\
\hline Longitud L(mm) & 6.5 & 8 \\
\hline
\end{tabular}

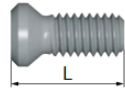

6) Compruebe que no existan espacios entre la placa y la base antes de empezar.

\section{Técnica para colocar la fresa al eje}

1) Antes de colocar la fresa al eje, limpie con cuidado la base y el extremo de la fresa, así como el extremo del eje

2) Coloque la fresa sobre el eje y apriete el tornillo de acople yue se suministra. Consulte el par de apriete en la tabla que aparece a continuación 3) El tornillo de acople que se suministra junto con la AXD es un tornillo especial para refrigeración interna Es importante que no lo pierda.

Tabla 1 Max. Revolución aceptable AXD4000

\begin{tabular}{c|c|c|c|c|c|c|c|c}
\hline Diámetro D1(mm) & $\varnothing 25$ & $\varnothing 32$ & $\varnothing 40$ & $\varnothing 50$ & $\varnothing 63$ & $\varnothing 80$ & $\varnothing 100$ & $\varnothing 125$ \\
\hline Max. Revoluciones (min-1) & 49000 & 48000 & 41000 & 35000 & 30000 & 27000 & 23000 & 20000 \\
\hline
\end{tabular}

Tabla 2 Máximas revoluciones cuando el equilibrado entre la barra ó el amarre no se consiguen AXD4000

\begin{tabular}{c|c|c|c|c|c|c|c|c}
\hline Diámetro D1(mm) & $\varnothing 25$ & $\varnothing 32$ & $\varnothing 40$ & $\varnothing 50$ & $\varnothing 63$ & $\varnothing 80$ & $\varnothing 100$ & $\varnothing 125$ \\
\hline Max. Revoluciones (min-1) & 12000 & 9500 & 7600 & 6000 & 4800 & 3800 & 3000 & 2400 \\
\hline
\end{tabular}

Cuando preparamos la velocidad del husillo, tener cuidado en considerar las máximas revoluciones permitidas de la barra ó amarre.

Usar el tornillo de fijación especifico cuando usamos el tipo con barra sin refrigeración.

Las placas tienen los filos de corte afilados, y pueden causar heridas cuando lo manipulamos con las manos. Utilizar siempre guantes de seguridad cuando manipulamos las placas.

\section{CONDICIONES DE CORTE RECOMENDADAS}

\begin{tabular}{|c|c|c|c|c|c|c|c|c|c|c|c|}
\hline \multirow{3}{*}{\multicolumn{2}{|c|}{ Material }} & \multirow{3}{*}{ Calidad } & \multirow{3}{*}{ Rompevicitas } & \multirow{3}{*}{$\begin{array}{c}\text { Velocidad de corte } \\
\text { vc }(\mathrm{m} / \mathrm{min})\end{array}$} & \multirow{3}{*}{$\begin{array}{c}\text { Ancho de corte } \\
\text { ae }(\mathrm{mm})\end{array}$} & \multirow{3}{*}{$\begin{array}{c}\text { Profinndidad de corte } \\
\text { ap }(\mathrm{mm})\end{array}$} & \multicolumn{5}{|c|}{ Avance por diente (mm/diente) } \\
\hline & & & & & & & \multicolumn{5}{|c|}{ Diametro del filo de corte D1 } \\
\hline & & & & & & & $\phi 20$ & $\phi 25-\phi 28$ & $\phi 32-\phi 35$ & $\phi 40$ & $\phi 50-\phi 125$ \\
\hline \multirow{12}{*}{$\begin{array}{l}\text { Aleación de aluminio } \\
\text { (A6061, A7075) }\end{array}$} & \multirow{12}{*}{ Si<5\% } & \multirow{12}{*}{$\begin{array}{c}\text { TF15 } \\
\text { LC15TF }\end{array}$} & \multirow{12}{*}{ GL } & \multirow{12}{*}{$\begin{array}{c}1000 \\
(200-3000)\end{array}$} & \multirow{3}{*}{$<0.25 \mathrm{D} 1$} & $<5$ & $<0.05$ & \multicolumn{4}{|c|}{$<0.25$} \\
\hline & & & & & & $<10$ & $<0.05$ & \multicolumn{4}{|c|}{$<0.2$} \\
\hline & & & & & & $<14.5$ & $<0.05$ & \multirow{2}{*}{\multicolumn{4}{|c|}{$\begin{array}{l}<0.15 \\
<0.25\end{array}$}} \\
\hline & & & & & \multirow{3}{*}{$<0.5 \mathrm{D} 1$} & $<5$ & $<0.05$ & & & & \\
\hline & & & & & & $<10$ & - & \multicolumn{4}{|c|}{$<0.2$} \\
\hline & & & & & & $<14.5$ & - & \multirow{2}{*}{\multicolumn{4}{|c|}{$\begin{array}{l}<0.15 \\
<0.25\end{array}$}} \\
\hline & & & & & \multirow{3}{*}{$<0.75 \mathrm{D} 1$} & $<5$ & $<0.05$ & \multicolumn{2}{|c|}{$<0.25$} & & \\
\hline & & & & & & $<10$ & - & \multirow{2}{*}{\multicolumn{4}{|c|}{$<0.2$}} \\
\hline & & & & & & $<14.5$ & - & \multirow{2}{*}{\multicolumn{4}{|c|}{$\begin{array}{l}<0.15 \\
<0.25\end{array}$}} \\
\hline & & & & & \multirow{3}{*}{ D1 } & $<5$ & $<0.05$ & & & & \\
\hline & & & & & & $<10$ & - & \multicolumn{4}{|c|}{-} \\
\hline & & & & & & $<14.5$ & - & \multicolumn{4}{|c|}{-} \\
\hline
\end{tabular}

(Nota 1) Las condiciones de corte de arriba estan basadas con alta rigidez de la pieza y máquina, donde no se produce vibración. Si se producen vibraciones realizar los ajustes según la condiciones de mecanizado

(Note 2) Las vibraciones pueden producirse en las siguientes condiciones.

Cuando utilizamos un voladizo largo.

Cuando realizamos un cajeado con fresas con radio

Cuando la pieza tiene una pobre sujección o cuando la rigidez de la máquina o pieza es baja,

si es así, reducir las condiciones de cortetales como el ancho de corte y el avance por diente. 


\section{I.5. ESPECIFICACIONES RUGOSÍMETRO SJ-201}

Surface Roughness Tester. Mitutoyo.

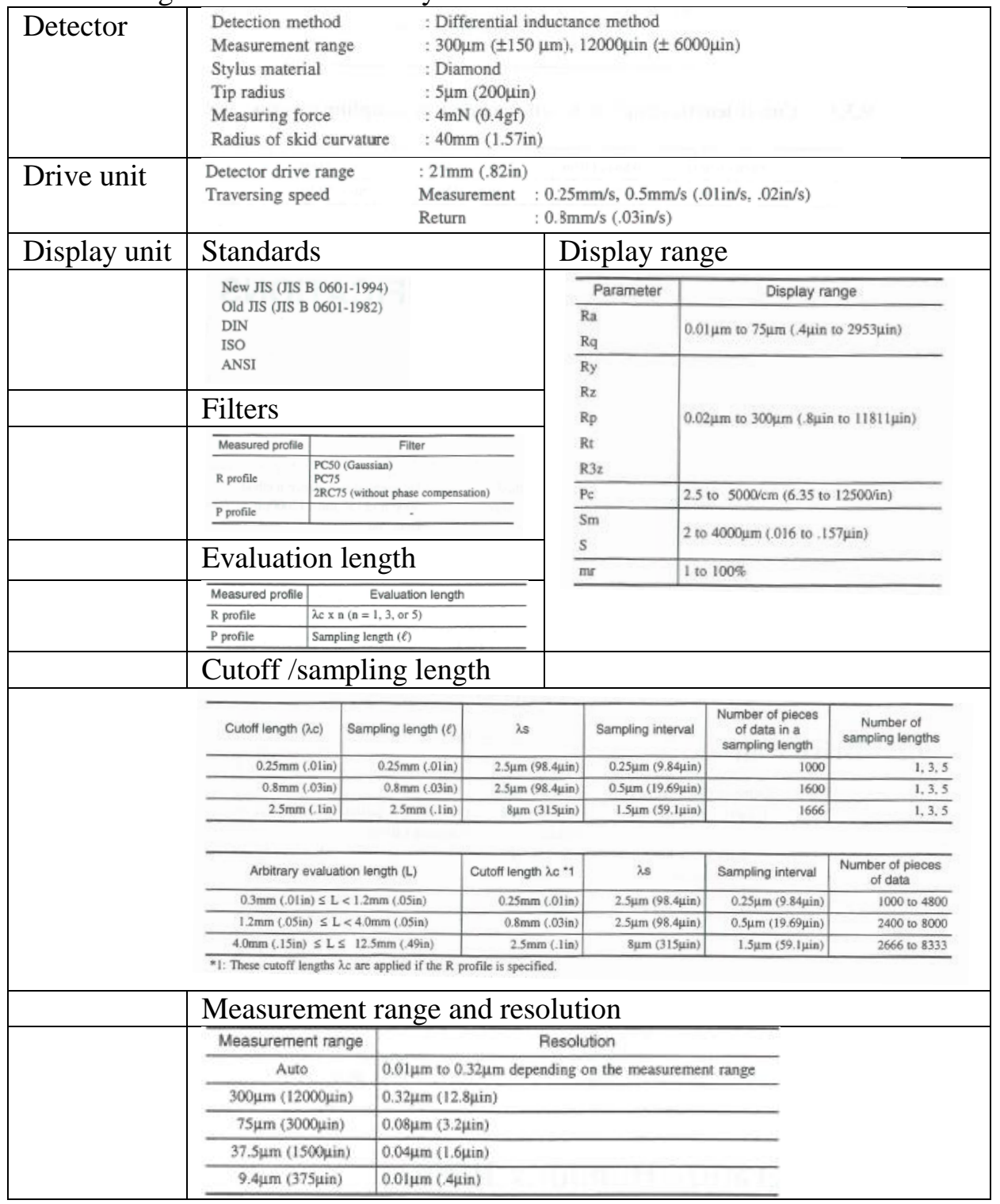




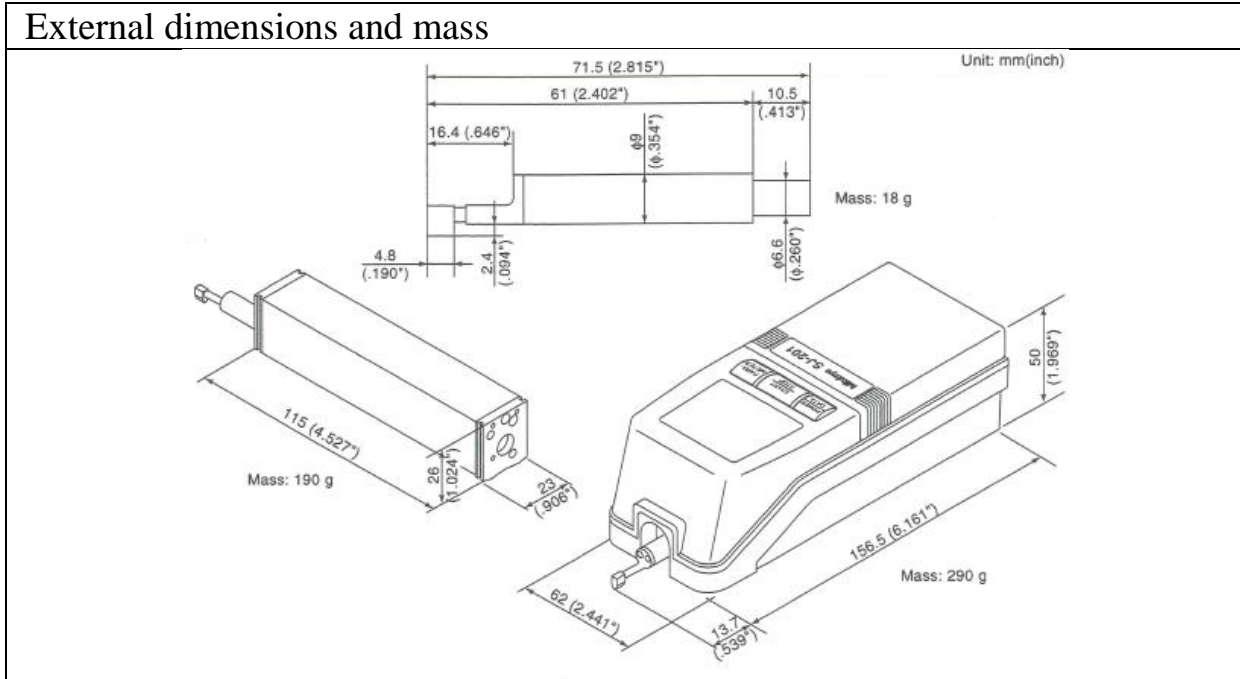

\section{I.6. EJEMPLO DE SALIDA DE DATOS - IMAGEN Vb}

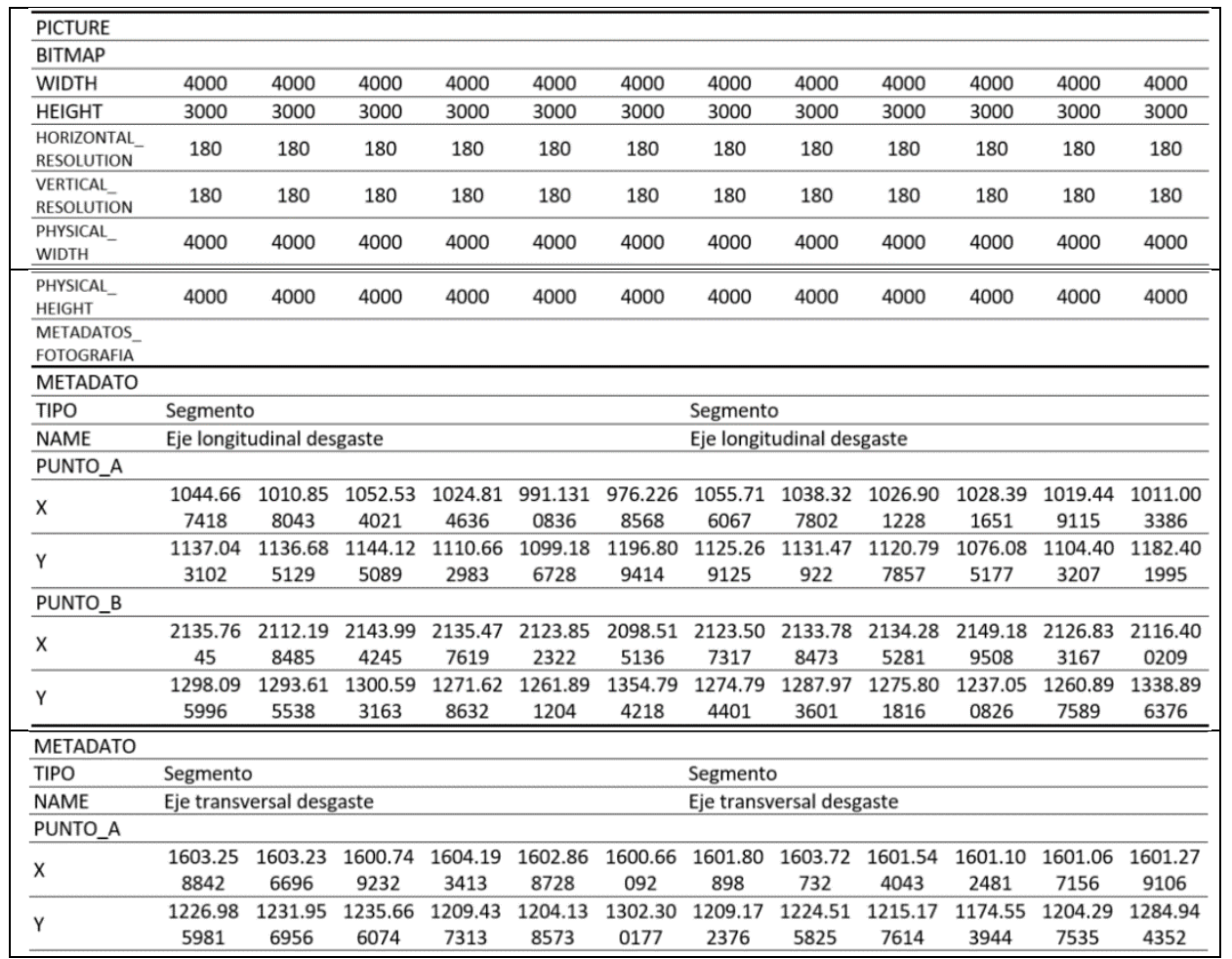


ANEXOS

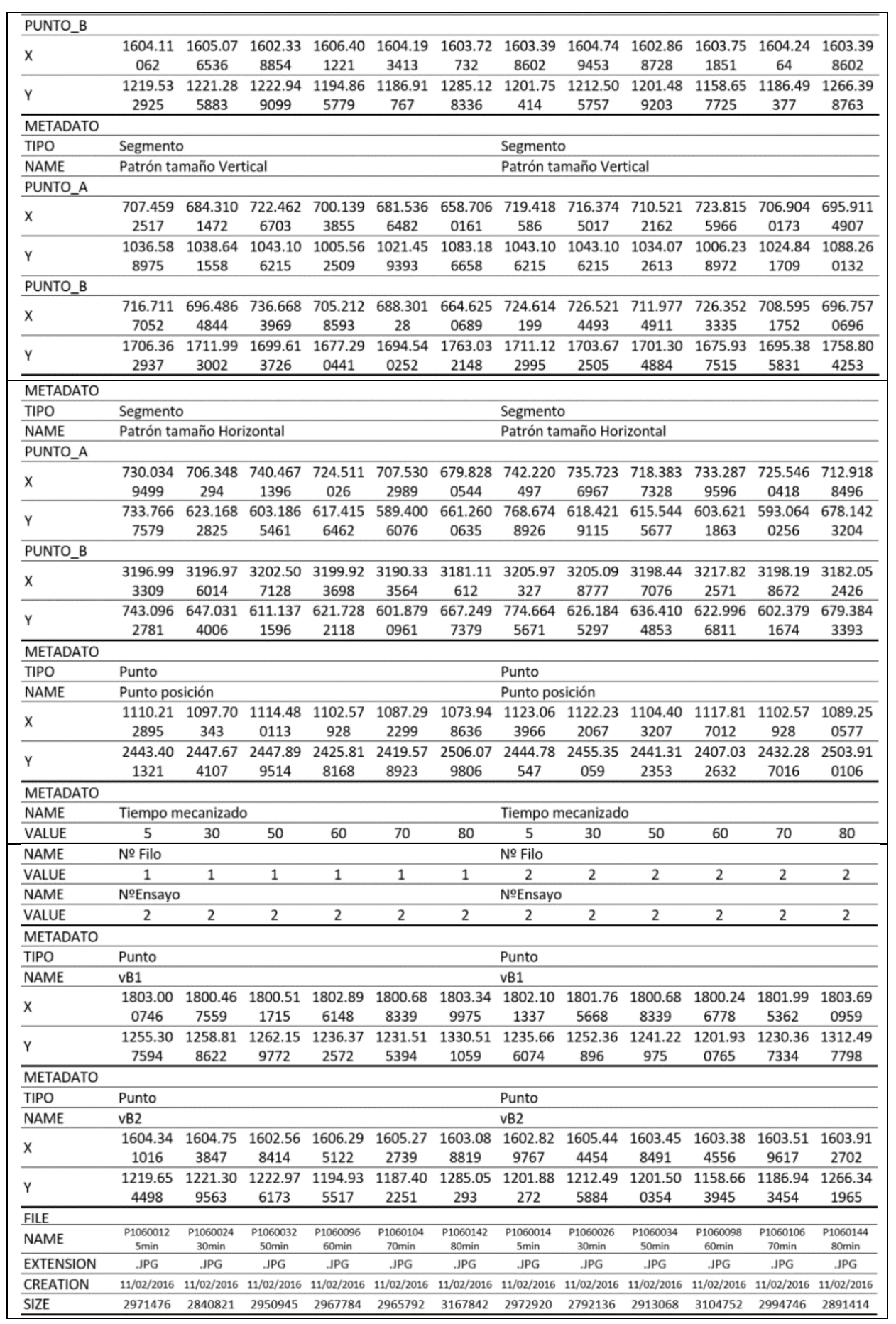




\section{ANEXOS AL CAPÍTULO 3}

\section{II.1. MEDIDAS DE RUGOSIDAD SUPERFICIAL (Ra)}

EXPERIMENTOS CON $\mathrm{Fv}=40 \%$

\begin{tabular}{ccccccccccccc}
\hline E01 & Tc 5 min & \multicolumn{2}{c}{ Tc $30 \mathrm{~min}$} & \multicolumn{2}{c}{ Tc $50 \mathrm{~min}$} & \multicolumn{2}{c}{ Tc $60 \mathrm{~min}$} & \multicolumn{2}{c}{ Tc $70 \mathrm{~min}$} & \multicolumn{2}{c}{ Tc $80 \mathrm{~min}$} \\
\hline Fv 40\% & $\mathrm{Ra}$ & $\mathrm{Rz}$ & $\mathrm{Ra}$ & $\mathrm{Rz}$ & $\mathrm{Ra}$ & $\mathrm{Rz}$ & $\mathrm{Ra}$ & $\mathrm{Rz}$ & $\mathrm{Ra}$ & $\mathrm{Rz}$ & $\mathrm{Ra}$ & $\mathrm{Rz}$ \\
\hline 1 & 4.44 & 27.1 & 3.74 & 28.33 & 3.79 & 22.35 & 3.30 & 23.5 & 3.53 & 20.89 & 4.25 & 26.07 \\
\hline 2 & 2.08 & 13.47 & 2.96 & 17.40 & 3.63 & 25.12 & 3.40 & 20.9 & 2.99 & 21.8 & 4.48 & 27.55 \\
\hline 3 & 2.79 & 18.23 & 2.57 & 14.87 & 3.46 & 22.28 & 3.17 & 15.81 & 5.59 & 37.78 & 5.17 & 32.90 \\
\hline 4 & 2.46 & 14.11 & 2.97 & 16.76 & 3.10 & 19.34 & 2.60 & 17.00 & 3.63 & 22.77 & 4.87 & 32.39 \\
\hline 5 & 2.49 & 15.32 & 3.05 & 16.86 & 3.28 & 20.9 & 3.70 & 19.67 & 3.19 & 22.35 & 3.85 & 25.74 \\
\hline 6 & 2.97 & 18.18 & 2.79 & 15.76 & 3.13 & 23.51 & 5.05 & 31.79 & 3.83 & 22.52 & 3.65 & 21.64 \\
\hline 7 & 2.53 & 19.27 & 2.93 & 17.01 & 3.62 & 23.12 & 5.17 & 32.15 & 4.82 & 29.02 & 4.96 & 29.45 \\
\hline 8 & 2.05 & 11.51 & 2.62 & 14.24 & 4.31 & 27.12 & 3.95 & 23.37 & 3.23 & 20.10 & 3.27 & 21.76 \\
\hline 9 & 1.89 & 13.82 & 2.81 & 19.73 & 3.03 & 17.69 & 4.02 & 24.33 & 5.50 & 37.71 & 3.62 & 26.55 \\
\hline 10 & 2.38 & 16.21 & 2.58 & 16.51 & 3.75 & 23.68 & 3.32 & 22.5 & 3.26 & 19.49 & 4.39 & 24.50 \\
\hline 11 & 2.60 & 14.79 & 2.16 & 13.29 & 4.34 & 25.93 & 4.87 & 34.14 & 4.47 & 28.59 & 3.21 & 20.95 \\
\hline 12 & 2.19 & 16.58 & 2.40 & 15.71 & 2.95 & 17.57 & 4.04 & 23.76 & 3.17 & 17.22 & 3.1 & 20.39 \\
\hline 13 & 2.62 & 15.12 & 2.35 & 14.64 & 3.36 & 20.80 & 2.99 & 17.92 & 3.85 & 22.78 & 5.42 & 31.18 \\
\hline 14 & 3.34 & 26.21 & 2.85 & 16.48 & 3.26 & 21.82 & 3.04 & 18.93 & 4.68 & 26.92 & 4.36 & 31.14 \\
\hline 15 & 2.94 & 19.21 & 3.34 & 21.55 & 3.69 & 21.68 & 4.74 & 27.79 & 2.95 & 19.10 & 4.83 & 28.63 \\
\hline media & 2.65 & 17.28 & 2.81 & 17.28 & 3.51 & 22.19 & 3.82 & 23.57 & 3.91 & 24.60 & 4.23 & 26.72 \\
\hline $\mathrm{s}$ & 0.63 & 4.41 & 0.40 & 3.69 & 0.42 & 2.74 & 0.82 & 5.68 & 0.89 & 6.30 & 0.75 & 4.24 \\
\hline
\end{tabular}

\begin{tabular}{|c|c|c|c|c|c|c|c|c|c|c|c|c|}
\hline E02 & \multicolumn{2}{|c|}{ Tc 5 min } & \multicolumn{2}{|c|}{ Tc $30 \mathrm{~min}$} & \multicolumn{2}{|c|}{ Tc $50 \mathrm{~min}$} & \multicolumn{2}{|c|}{ Tc $60 \mathrm{~min}$} & \multicolumn{2}{|c|}{ Tc $70 \mathrm{~min}$} & \multicolumn{2}{|c|}{ Tc $80 \mathrm{~min}$} \\
\hline Fv $40 \%$ & $\mathrm{Ra}$ & $\mathrm{Rz}$ & $\mathrm{Ra}$ & $\mathrm{Rz}$ & $\mathrm{Ra}$ & $\mathrm{Rz}$ & $\mathrm{Ra}$ & $\mathrm{Rz}$ & $\mathrm{Ra}$ & $\mathrm{Rz}$ & $\mathrm{Ra}$ & $\mathrm{Rz}$ \\
\hline 1 & 4.65 & 34.70 & 3.26 & 23.89 & 3.92 & 29.29 & 2.13 & 13.53 & 4.47 & 29.29 & 4.49 & 29.72 \\
\hline 2 & 3.89 & 27.05 & 4.58 & 29.81 & 4.64 & 29.85 & 4.59 & 0.6 & 6.17 & 40. & 5.29 & 29.28 \\
\hline 3 & 2.51 & 13.2 & 3.46 & .53 & 5.04 & 3. & 5.48 & .15 & 6.72 & 0. & .86 & 5.19 \\
\hline 4 & 2.19 & 14.8 & 4.34 & 5.63 & 4.43 & 32.74 & 7.28 & 41.5 & 3.25 & & 3.50 & 99.63 \\
\hline 5 & 2.86 & 20.1 & 2.90 & 23.16 & 2.60 & 16.88 & 6.56 & 38.45 & 5.28 & 34. & 4.91 & 28.14 \\
\hline 6 & 2.55 & 20.3 & 3.83 & 25.20 & 3.20 & 18.45 & 3.31 & 21.11 & 7.22 & 41.4 & 5.77 & 35.99 \\
\hline 7 & 2.31 & 16.91 & 6.41 & 36.48 & 5.56 & 34.76 & 3.48 & 21.41 & 5.68 & 31.6 & 4.28 & 27.28 \\
\hline 8 & 3.06 & 23.1 & 6.69 & 39.75 & 6.40 & 47.85 & 7.38 & 47.44 & 4.13 & 27. & 7.12 & 48.92 \\
\hline 9 & 4.35 & 29.0 & 4.96 & 35.85 & 6.46 & 40.42 & 7.97 & 50.6 & 6.83 & & 5.14 & 38.43 \\
\hline 10 & 3.32 & 24.73 & 4.15 & 27.88 & 4.02 & 26.23 & 5.80 & 34.21 & 6.84 & 34.53 & 7.00 & 37.21 \\
\hline 11 & 4.15 & 34.99 & 2.01 & 14.19 & 3.87 & 24.14 & 7.34 & 35.50 & 6.07 & 37.27 & 8.46 & 49.06 \\
\hline 12 & 2.92 & 23.01 & 2.08 & 14.32 & 3.94 & 22.74 & 3.70 & 27.16 & 4.20 & 27.80 & 6.13 & 37.57 \\
\hline 13 & 3.23 & 24.8 & 2.77 & 20.25 & 1.85 & 11.78 & 6.85 & 40.27 & 6.72 & 40.84 & 9.63 & 51.74 \\
\hline 14 & 6.22 & 36.1 & 3.57 & 22.69 & 3.87 & 22.38 & 6.09 & 36.25 & 5.68 & 40.00 & 6.46 & 45.82 \\
\hline 15 & 6.35 & 39.17 & 2.82 & 17.71 & 6.53 & 42.31 & 4.95 & 29.67 & 6.22 & 37.37 & 5.95 & 40.31 \\
\hline media & 3.64 & 25.49 & 3.86 & 26.16 & 4.42 & 28.76 & 5.53 & 33.67 & 5.70 & 35.31 & 6.27 & 38.95 \\
\hline $\mathrm{s}$ & 1.31 & 8.00 & 1.39 & 8.05 & 1.39 & 9.95 & 1.78 & 10.05 & 1.20 & 7.38 & 1.66 & 10.27 \\
\hline E03 & Tc 5 & & Tc 3 & $\min$ & Tc 5 & $\min$ & Тс 6 & IIIII & Tc 7 & & Tc 8 & $\min$ \\
\hline v $40 \%$ & $\mathrm{Ra}$ & & $a$ & $\mathrm{Rz}$ & $\mathrm{Ra}$ & $\mathrm{Rz}$ & $\mathrm{Ra}$ & $\mathrm{Rz}$ & $\mathrm{Ra}$ & $\mathrm{Rz}$ & $\mathrm{Ra}$ & $\mathrm{Rz}$ \\
\hline 1 & 2.58 & 17.14 & 4.60 & 31.62 & 2.92 & 22.66 & 4.69 & 30.02 & 5.49 & 33.32 & 3.13 & 20.27 \\
\hline
\end{tabular}


ANEXOS

\begin{tabular}{cccccccccccccc}
\hline E03 & \multicolumn{1}{c}{ Tc 5 min } & \multicolumn{1}{c}{$\mathrm{Tc} 30 \mathrm{~min}$} & \multicolumn{2}{c}{$\mathrm{Tc} 50 \mathrm{~min}$} & \multicolumn{2}{c}{$\mathrm{Tc} 60 \mathrm{~min}$} & \multicolumn{2}{c}{$\mathrm{Tc} 70 \mathrm{~min}$} & \multicolumn{2}{c}{ Tc $80 \mathrm{~min}$} \\
\hline Fv 40\% & $\mathrm{Ra}$ & $\mathrm{Rz}$ & $\mathrm{Ra}$ & $\mathrm{Rz}$ & $\mathrm{Ra}$ & $\mathrm{Rz}$ & $\mathrm{Ra}$ & $\mathrm{Rz}$ & $\mathrm{Ra}$ & $\mathrm{Rz}$ & $\mathrm{Ra}$ & $\mathrm{Rz}$ \\
\hline 2 & 2.67 & 20.52 & 4.06 & 29.92 & 3.04 & 21.94 & 4.08 & 27.34 & 3.77 & 23.45 & 5.55 & 28.43 \\
\hline 3 & 3.26 & 24.20 & 1.96 & 14.05 & 2.21 & 13.45 & 5.64 & 37.93 & 4.54 & 33.91 & 2.50 & 16.23 \\
\hline 4 & 3.65 & 22.60 & 3.39 & 20.37 & 2.41 & 14.15 & 3.19 & 21.10 & 4.18 & 27.70 & 5.43 & 37.45 \\
\hline 5 & 2.01 & 15.10 & 2.57 & 18.72 & 5.74 & 32.93 & 2.95 & 19.33 & 4.86 & 26.09 & 5.02 & 31.77 \\
\hline 6 & 4.08 & 27.76 & 3.49 & 26.12 & 5.93 & 33.61 & 5.99 & 41.30 & 4.65 & 29.01 & 3.13 & 19.95 \\
\hline 7 & 2.96 & 20.22 & 2.44 & 18.48 & 5.36 & 32.90 & 5.54 & 37.89 & 3.99 & 22.94 & 6.71 & 35.09 \\
\hline 8 & 2.07 & 20.86 & 2.52 & 15.27 & 2.45 & 13.41 & 5.11 & 32.72 & 5.34 & 42.63 & 4.80 & 29.82 \\
\hline 9 & 2.86 & 20.39 & 3.97 & 28.43 & 3.25 & 20.62 & 2.70 & 18.39 & 2.02 & 14.05 & 3.53 & 21.97 \\
\hline 10 & 2.22 & 14.44 & 1.90 & 13.59 & 5.14 & 42.34 & 5.66 & 34.15 & 6.28 & 35.63 & 4.20 & 26.82 \\
\hline 11 & 2.28 & 14.20 & 3.77 & 23.36 & 4.05 & 24.12 & 4.41 & 26.48 & 3.36 & 21.89 & 6.03 & 41.09 \\
\hline 12 & 5.05 & 37.59 & 1.97 & 13.09 & 4.24 & 26.92 & 3.22 & 23.70 & 6.36 & 35.27 & 4.66 & 30.88 \\
\hline 13 & 2.31 & 13.80 & 2.48 & 15.95 & 2.94 & 19.09 & 3.55 & 21.39 & 4.57 & 27.35 & 5.16 & 34.09 \\
\hline 14 & 2.66 & 17.25 & 3.04 & 23.16 & 4.52 & 27.66 & 4.86 & 30.35 & 2.76 & 17.37 & 4.24 & 27.51 \\
\hline 15 & 2.77 & 18.12 & 1.79 & 10.00 & 3.30 & 20.70 & 3.39 & 26.56 & 3.49 & 23.96 & 2.63 & 17.63 \\
\hline media & 2.90 & 20.28 & 2.93 & 20.14 & 3.83 & 24.43 & 4.33 & 28.58 & 4.38 & 27.64 & 4.45 & 27.93 \\
\hline $\mathrm{s}$ & 0.83 & 6.21 & 0.90 & 6.71 & 1.26 & 8.37 & 1.11 & 7.17 & 1.21 & 7.56 & 1.26 & 7.48 \\
\hline
\end{tabular}

\begin{tabular}{|c|c|c|c|c|c|c|c|c|c|c|c|c|}
\hline E06 & \multicolumn{2}{|c|}{ Tc 5 min } & \multicolumn{2}{|c|}{ Tc $30 \mathrm{~min}$} & \multicolumn{2}{|c|}{ Tc $50 \mathrm{~min}$} & \multicolumn{2}{|c|}{ Tc $60 \mathrm{~min}$} & \multicolumn{2}{|c|}{ Tc $70 \mathrm{~min}$} & \multicolumn{2}{|c|}{ Tc $80 \mathrm{~min}$} \\
\hline Fv 40\% & $\mathrm{Ra}$ & $\mathrm{Rz}$ & $\mathrm{Ra}$ & $\mathrm{Rz}$ & $\mathrm{Ra}$ & $\mathrm{Rz}$ & $\mathrm{Ra}$ & $\mathrm{Rz}$ & $\mathrm{Ra}$ & $\mathrm{Rz}$ & $\mathrm{Ra}$ & $\mathrm{Rz}$ \\
\hline 1 & 3.30 & 18.81 & 3.42 & 20.22 & 3.57 & 19.78 & 3.74 & 24.92 & 3.16 & 19.53 & 5.74 & 30.22 \\
\hline 2 & 2.85 & 19.56 & 2.96 & 17.92 & 3.97 & 21.68 & 3.28 & 20.92 & 5.08 & 29.95 & 3.17 & 22.10 \\
\hline 3 & 2.90 & 17.67 & 3.88 & 21.74 & 3.05 & 18.03 & 3.49 & 19.59 & 3.60 & 23.30 & 5.87 & 33.70 \\
\hline 4 & 3.28 & 20.32 & 3.65 & 21.78 & 4.45 & 26.69 & 2.76 & 16.79 & 4.00 & 20.49 & 3.39 & 23.04 \\
\hline 5 & 3.70 & 23.25 & 2.31 & 14.54 & 2.26 & 14.66 & 3.74 & 21.33 & 3.51 & 21.20 & 3.23 & 20.06 \\
\hline 6 & 2.43 & 14.15 & 4.63 & 32.41 & 3.73 & 20.94 & 3.67 & 19.98 & 3.90 & 24.12 & 7.77 & 39.06 \\
\hline 7 & 4.19 & 28.49 & 4.70 & 30.44 & 4.50 & 29.20 & 3.53 & 18.31 & 4.49 & 26.64 & 3.69 & 26.71 \\
\hline 8 & 3.04 & 17.62 & 4.35 & 25.85 & 5.44 & 28.12 & 3.33 & 20.78 & 4.89 & 28.87 & 3.47 & 21.87 \\
\hline 9 & 5.83 & 35.19 & 2.74 & 16.82 & 4.03 & 23.18 & 4.07 & 27.76 & 2.63 & 18.38 & 3.46 & 21.83 \\
\hline 10 & 3.11 & 16.93 & 3.39 & 20.87 & 4.66 & 29.25 & 3.32 & 22.23 & 3.14 & 18.74 & 3.64 & 21.33 \\
\hline 11 & 3.78 & 23.24 & 4.00 & 25.89 & 3.36 & 20.17 & 5.45 & 41.32 & 5.16 & 31.44 & 3.60 & 22.14 \\
\hline 12 & 3.31 & 19.93 & 5.68 & 36.12 & 3.30 & 20.14 & 3.05 & 19.62 & 3.76 & 21.76 & 3.29 & 19.21 \\
\hline 13 & 3.27 & 18.75 & 3.13 & 20.91 & 3.14 & 20.59 & 5.34 & 38.16 & 4.21 & 27.82 & 3.95 & 26.85 \\
\hline 14 & 2.95 & 17.66 & 4.09 & 27.13 & 3.59 & 22.23 & 2.73 & 18.46 & 4.20 & 31.99 & 3.68 & 22.50 \\
\hline 15 & 3.21 & 18.91 & 3.34 & 21.63 & 3.81 & 27.12 & 4.99 & 31.41 & 3.65 & 22.60 & 2.74 & 18.35 \\
\hline media & 3.41 & 20.70 & 3.75 & 23.62 & 3.79 & 22.79 & 3.77 & 24.11 & 3.96 & 24.46 & 4.05 & 24.60 \\
\hline $\mathrm{s}$ & 0.79 & 5.22 & 0.87 & 5.98 & 0.77 & 4.37 & 0.86 & 7.42 & 0.73 & 4.66 & 1.35 & 5.77 \\
\hline E09 & \multicolumn{2}{|c|}{ Tc 5 min } & \multicolumn{2}{|c|}{ Tc $30 \mathrm{~min}$} & \multicolumn{2}{|c|}{ Tc $50 \mathrm{~min}$} & \multicolumn{2}{|c|}{ Tc $60 \mathrm{~min}$} & \multicolumn{2}{|c|}{ Tc $70 \mathrm{~min}$} & \multicolumn{2}{|c|}{ Tc $80 \mathrm{~min}$} \\
\hline Fv 40\% & $\mathrm{Ra}$ & $\mathrm{Rz}$ & $\mathrm{Ra}$ & $\mathrm{Rz}$ & $\mathrm{Ra}$ & $\mathrm{Rz}$ & $\mathrm{Ra}$ & $\mathrm{Rz}$ & $\mathrm{Ra}$ & $\mathrm{Rz}$ & $\mathrm{Ra}$ & $\mathrm{Rz}$ \\
\hline 1 & 3.95 & 25.16 & 3.91 & 29.02 & 4.54 & 28.03 & 4.69 & 28.01 & 5.84 & 35.62 & 3.81 & 22.68 \\
\hline 2 & 3.59 & 23.16 & 3.91 & 26.58 & 3.58 & 22.29 & 5.49 & 34.08 & 3.74 & 22.82 & 6.66 & 37.26 \\
\hline 3 & 3.87 & 25.90 & 4.39 & 36.32 & 3.52 & 21.46 & 3.29 & 23.05 & 3.34 & 25.52 & 4.84 & 30.79 \\
\hline 4 & 1.45 & 10.46 & 4.76 & 26.83 & 3.13 & 22.92 & 2.44 & 14.50 & 3.79 & 25.27 & 2.42 & 14.52 \\
\hline 5 & 4.72 & 25.63 & 2.05 & 13.13 & 3.06 & 20.88 & 2.85 & 15.57 & 3.13 & 15.47 & 3.28 & 22.83 \\
\hline 6 & 3.90 & 24.34 & 4.82 & 23.43 & 3.05 & 21.41 & 6.02 & 37.65 & 3.14 & 20.36 & 5.42 & 30.44 \\
\hline 7 & 3.80 & 27.20 & 3.23 & 21.07 & 4.22 & 26.66 & 2.93 & 21.73 & 4.31 & 27.21 & 3.79 & 21.77 \\
\hline 8 & 2.15 & 14.96 & 5.52 & 31.67 & 2.34 & 15.41 & 3.70 & 25.61 & 4.76 & 29.79 & 5.65 & 35.03 \\
\hline 9 & 1.27 & 9.33 & 3.28 & 23.43 & 4.87 & 28.07 & 3.84 & 24.78 & 4.49 & 35.51 & 3.58 & 24.84 \\
\hline
\end{tabular}




\begin{tabular}{|c|c|c|c|c|c|c|c|c|c|c|c|c|}
\hline E09 & \multicolumn{2}{|c|}{ Tc 5 min } & \multicolumn{2}{|c|}{ Tc $30 \mathrm{~min}$} & \multicolumn{2}{|c|}{ Tc $50 \mathrm{~min}$} & \multicolumn{2}{|c|}{ Tc $60 \mathrm{~min}$} & \multicolumn{2}{|c|}{ Tc $70 \mathrm{~min}$} & \multicolumn{2}{|c|}{ Tc $80 \mathrm{~min}$} \\
\hline V $40 \%$ & $\mathrm{Ra}$ & $\mathrm{Rz}$ & $\mathrm{Ra}$ & $\mathrm{Rz}$ & $\mathrm{Ra}$ & $\mathrm{Rz}$ & $\mathrm{Ra}$ & $\mathrm{Rz}$ & $\mathrm{Ra}$ & $\mathrm{Rz}$ & $\mathrm{Ra}$ & $\mathrm{Rz}$ \\
\hline 10 & 4.91 & 26.57 & 2.96 & 22.72 & 4.90 & 27.31 & 3.53 & 27.46 & 5.58 & 38.11 & 6.38 & 35.79 \\
\hline 11 & 2.70 & 18.42 & .53 & 23.76 & 2.98 & 20.45 & 5.31 & 30.92 & 3.28 & 22.40 & 3.83 & 23.27 \\
\hline 12 & 2.84 & 20.36 & 3.18 & 21.72 & 4.83 & 28.88 & .32 & 33.52 & 4.75 & 8 & 3.32 & 20.03 \\
\hline 13 & 34 & 36.34 & 43 & 23.99 & 4.50 & 33.53 & .24 & 9.91 & 3.99 & 40 & .53 & 0.54 \\
\hline 14 & 89 & 27.3 & 61 & 26.92 & 3.82 & 25.35 & 8.74 & 0.0 & 4.17 & 0 & 3.23 & 0.52 \\
\hline 15 & 1.02 & 6.46 & 3.33 & 19.57 & 3.63 & 24.47 & 3.38 & 23.76 & 4.16 & 0.83 & 6.26 & 39.37 \\
\hline media & 3.29 & 21.44 & 3.73 & 24.68 & 3.80 & 24.47 & 4.12 & 26.44 & 4.16 & 26.41 & 4.40 & 26.65 \\
\hline $\mathrm{s}$ & 1.34 & 8.12 & 0.86 & 5.39 & 0.81 & 4.45 & 1.19 & 7.06 & 0.83 & 6.33 & 1.35 & 7.53 \\
\hline E10 & Tc 5 & & Tc 3 & & Tc & $\min$ & Tc 6 & $\min$ & & $\min$ & & $\min$ \\
\hline v $40 \%$ & $\mathrm{Ra}$ & $\mathrm{Rz}$ & $\mathrm{Ra}$ & $\mathrm{Rz}$ & $\mathrm{Ra}$ & $\mathrm{Rz}$ & $\mathrm{Ra}$ & $\mathrm{Rz}$ & $\mathrm{Ra}$ & $\mathrm{Rz}$ & $\mathrm{Ra}$ & $\mathrm{Rz}$ \\
\hline 1 & 1.83 & 10.17 & 2.18 & 14.98 & 3.48 & 19.45 & 3.67 & 21.38 & 4.59 & 24.14 & 7.04 & 43.39 \\
\hline 2 & 1.06 & 6.64 & 4.15 & 25.54 & 1.33 & 8.51 & 4.72 & 27.39 & 2.77 & 16.09 & 3.64 & 19.97 \\
\hline 3 & 3.81 & 19.98 & 2.55 & 16.18 & 2.57 & 13.65 & 4.24 & 31.39 & 3.55 & 21.12 & 6.05 & 30.73 \\
\hline 4 & 3.22 & 18.28 & 1.24 & 7.28 & 3.35 & 21.88 & 2.75 & 19.41 & 4.91 & 27.73 & 4.11 & 26.10 \\
\hline 5 & 2.46 & 14.49 & 3.24 & 18.72 & 3.21 & 18.59 & 3.46 & 18.26 & 2.54 & 16.72 & 4.13 & 25.79 \\
\hline 6 & 1.69 & 11.2 & 2.24 & 18.03 & 4.06 & 26.17 & 3.19 & 17.06 & 3.76 & 20.14 & 4.95 & 31.67 \\
\hline 7 & 1.43 & 11.33 & 2.57 & 18.62 & 3.92 & 25.67 & 5.00 & 28.98 & 3.41 & 20.75 & 5.31 & 32.29 \\
\hline 8 & 48 & 18.15 & 1.36 & 8.23 & 2.52 & 17.09 & 3.11 & 19.55 & 2.87 & 16.34 & 6.01 & 37.15 \\
\hline 9 & 2.04 & 13.78 & 2.59 & 14.64 & 2.55 & 19.32 & 3.27 & 20.81 & 4.54 & 29.40 & 2.89 & 17.09 \\
\hline 10 & 15 & & 3.37 & 19.93 & 3.60 & 28.07 & 2.72 & 16.18 & 3.62 & 23.77 & 3.93 & 25.02 \\
\hline 11 & 66 & 1 & 2.63 & 18.50 & 3.37 & 20.30 & 3.30 & 24.86 & 3.98 & 26.88 & 4.31 & 24.85 \\
\hline 12 & 1.79 & 11.39 & 2.67 & 15.43 & 2.96 & 17.13 & 2.79 & 17.33 & 4.94 & 32.70 & 3.61 & 21.98 \\
\hline 13 & 1.78 & 2.78 & 2.32 & 16.25 & 3.49 & 19.39 & 2.41 & 14.45 & 2.57 & 16.74 & 3.51 & 19.93 \\
\hline 14 & 2.03 & 11.21 & 3.57 & 22.72 & 3.77 & 23.07 & 3.41 & 21.60 & 4.40 & 27.80 & 3.75 & 26.14 \\
\hline 15 & & & 3 & 16.18 & 2.41 & 16.00 & 3.56 & 20.81 & 1.66 & 10.02 & 4.19 & 26.27 \\
\hline media & 1.96 & 12.3 & 2.3 & 16.75 & 3.1 & 19.62 & 3.44 & 21.30 & 3.61 & 22.02 & 4.50 & 27.22 \\
\hline $\mathrm{s}$ & 0.78 & 4.13 & 0.77 & 4.69 & 0.72 & 5.03 & 0.73 & 4.88 & 0.98 & 6.21 & 1.15 & 6.92 \\
\hline
\end{tabular}

$\begin{array}{lllllll}\text { E13 } & \text { Tc } 5 \mathrm{~min} & \text { Tc } 30 \mathrm{~min} & \text { Tc } 50 \mathrm{~min} & \text { Tc } 60 \mathrm{~min} & \text { Tc } 70 \mathrm{~min} & \text { Tc } 80 \mathrm{~min}\end{array}$

\begin{tabular}{ccccccccccccc}
\hline Fv 40\% & $\mathrm{Ra}$ & $\mathrm{Rz}$ & $\mathrm{Ra}$ & $\mathrm{Rz}$ & $\mathrm{Ra}$ & $\mathrm{Rz}$ & $\mathrm{Ra}$ & $\mathrm{Rz}$ & $\mathrm{Ra}$ & $\mathrm{Rz}$ & $\mathrm{Ra}$ & $\mathrm{Rz}$ \\
\hline 1 & 4.73 & 26.96 & 4.17 & 24.24 & 4.82 & 27.25 & 5.43 & 32.84 & 7.71 & 49.42 & 6.97 & 46.91 \\
\hline 2 & 4.72 & 21.97 & 6.52 & 37.72 & 7.00 & 35.25 & 4.59 & 25.97 & 6.50 & 37.46 & 9.71 & 47.13 \\
\hline 3 & 4.84 & 29.39 & 9.51 & 49.26 & 6.81 & 39.38 & 5.08 & 32.82 & 5.34 & 39.31 & 4.00 & 23.06 \\
\hline 4 & 6.16 & 40.90 & 5.97 & 36.24 & 4.56 & 29.89 & 6.46 & 41.85 & 9.54 & 54.47 & 5.80 & 36.20 \\
\hline 5 & 3.83 & 29.28 & 5.13 & 31.80 & 4.27 & 26.02 & 7.65 & 49.72 & 7.19 & 43.36 & 6.67 & 39.21 \\
\hline 6 & 6.17 & 36.34 & 2.89 & 19.70 & 7.76 & 47.11 & 6.38 & 32.13 & 7.78 & 42.80 & 6.80 & 35.74 \\
\hline 7 & 5.97 & 35.19 & 4.37 & 27.20 & 6.99 & 47.05 & 6.69 & 42.43 & 9.41 & 58.82 & 6.50 & 37.72 \\
\hline 8 & 6.99 & 36.35 & 5.49 & 31.70 & 7.54 & 49.04 & 5.29 & 31.96 & 5.88 & 38.44 & 10.57 & 57.93 \\
\hline 9 & 5.55 & 30.65 & 7.38 & 46.26 & 5.86 & 38.01 & 7.85 & 38.76 & 5.79 & 32.86 & 4.32 & 30.67 \\
\hline 10 & 5.19 & 31.10 & 6.26 & 37.32 & 5.08 & 29.32 & 5.22 & 41.01 & 3.95 & 22.57 & 6.35 & 37.41 \\
\hline 11 & 3.97 & 25.35 & 6.38 & 39.77 & 4.28 & 27.33 & 6.81 & 50.99 & 6.16 & 38.68 & 9.76 & 53.66 \\
\hline 12 & 4.38 & 26.45 & 6.08 & 35.62 & 5.68 & 31.24 & 7.33 & 45.18 & 6.22 & 38.95 & 4.25 & 28.08 \\
\hline 13 & 5.88 & 31.61 & 4.97 & 30.04 & 7.97 & 51.99 & 6.38 & 47.28 & 7.10 & 43.08 & 10.21 & 63.81 \\
\hline 14 & 5.95 & 31.32 & 5.95 & 32.64 & 6.29 & 35.01 & 5.34 & 34.81 & 10.17 & 55.36 & 5.53 & 31.33 \\
\hline 15 & 6.35 & 39.16 & 6.20 & 40.31 & 9.50 & 49.69 & 8.50 & 46.41 & 6.08 & 34.33 & 8.29 & 46.90 \\
\hline media & 5.38 & 31.47 & 5.82 & 34.65 & 6.29 & 37.57 & 6.33 & 39.61 & 6.99 & 41.99 & 7.05 & 41.05 \\
\hline $\mathrm{s}$ & 0.94 & 5.30 & 1.51 & 7.82 & 1.54 & 9.24 & 1.16 & 7.55 & 1.70 & 9.47 & 2.20 & 11.47 \\
\hline
\end{tabular}




\begin{tabular}{cccccccccccccc}
\hline E15 & \multicolumn{1}{c}{ Tc $5 \mathrm{~min}$} & \multicolumn{2}{c}{$\mathrm{Tc} 30 \mathrm{~min}$} & \multicolumn{2}{c}{ Tc $50 \mathrm{~min}$} & \multicolumn{2}{c}{ Tc $60 \mathrm{~min}$} & \multicolumn{2}{l}{ Tc $70 \mathrm{~min}$} & \multicolumn{2}{c}{ Tc $80 \mathrm{~min}$} \\
\hline Fv 40\% & $\mathrm{Ra}$ & $\mathrm{Rz}$ & $\mathrm{Ra}$ & $\mathrm{Rz}$ & $\mathrm{Ra}$ & $\mathrm{Rz}$ & $\mathrm{Ra}$ & $\mathrm{Rz}$ & $\mathrm{Ra}$ & $\mathrm{Rz}$ & $\mathrm{Ra}$ & $\mathrm{Rz}$ \\
\hline 1 & 3.52 & 21.95 & 2.91 & 18.86 & 3.79 & 20.82 & 2.52 & 14.61 & 3.73 & 20.67 & 4.03 & 32.10 \\
\hline 2 & 2.76 & 17.25 & 5.49 & 42.58 & 2.96 & 18.51 & 3.56 & 19.26 & 3.39 & 21.07 & 5.29 & 32.37 \\
\hline 3 & 3.90 & 27.22 & 4.26 & 25.41 & 3.67 & 19.73 & 2.95 & 15.82 & 3.63 & 25.31 & 4.40 & 26.92 \\
\hline 4 & 2.15 & 15.73 & 3.15 & 19.05 & 2.50 & 16.08 & 2.99 & 17.65 & 5.58 & 32.33 & 5.12 & 34.52 \\
\hline 5 & 2.97 & 18.99 & 2.98 & 14.67 & 4.31 & 28.61 & 4.05 & 27.42 & 5.14 & 27.93 & 5.08 & 28.47 \\
\hline 6 & 2.64 & 17.52 & 2.18 & 16.75 & 4.00 & 22.76 & 3.18 & 19.33 & 3.78 & 20.94 & 3.23 & 17.17 \\
\hline 7 & 3.76 & 20.10 & 2.15 & 14.16 & 3.87 & 20.58 & 4.47 & 25.37 & 3.46 & 19.46 & 3.40 & 19.79 \\
\hline 8 & 3.58 & 23.89 & 2.40 & 15.80 & 5.24 & 38.12 & 4.52 & 23.57 & 3.50 & 23.23 & 4.02 & 24.80 \\
\hline 9 & 3.60 & 19.42 & 3.07 & 18.34 & 3.36 & 20.81 & 2.93 & 19.68 & 2.75 & 17.95 & 4.91 & 31.15 \\
\hline 10 & 2.58 & 13.71 & 3.11 & 17.02 & 2.21 & 11.69 & 3.38 & 20.20 & 3.22 & 21.71 & 2.80 & 14.71 \\
\hline 11 & 3.80 & 23.17 & 2.33 & 14.50 & 3.25 & 23.21 & 3.42 & 21.82 & 2.69 & 15.64 & 3.27 & 20.65 \\
\hline 12 & 3.07 & 19.65 & 3.33 & 22.68 & 3.31 & 18.41 & 4.20 & 23.23 & 3.05 & 16.90 & 3.90 & 23.22 \\
\hline 13 & 3.06 & 18.10 & 2.94 & 20.12 & 4.26 & 21.71 & 3.65 & 19.31 & 2.53 & 14.69 & 2.37 & 15.25 \\
\hline 14 & 3.44 & 22.32 & 4.40 & 24.32 & 2.75 & 15.00 & 3.60 & 20.33 & 3.80 & 23.48 & 3.49 & 21.32 \\
\hline 15 & 2.56 & 16.88 & 3.60 & 20.92 & 2.80 & 20.43 & 3.49 & 26.64 & 4.72 & 31.79 & 2.94 & 20.44 \\
\hline media & 3.16 & 19.73 & 3.22 & 20.35 & 3.49 & 21.10 & 3.53 & 20.95 & 3.66 & 22.21 & 3.88 & 24.19 \\
\hline $\mathrm{s}$ & 0.54 & 3.50 & 0.92 & 7.07 & 0.80 & 6.10 & 0.58 & 3.73 & 0.88 & 5.33 & 0.92 & 6.46 \\
\hline
\end{tabular}

EXPERIMENTOS CON Fv $=60 \%$

\begin{tabular}{|c|c|c|c|c|c|c|c|c|c|c|c|c|}
\hline E04 & \multicolumn{2}{|c|}{ Tc 5 min } & \multicolumn{2}{|c|}{ Tc $30 \mathrm{~min}$} & \multicolumn{2}{|c|}{ Tc $50 \mathrm{~min}$} & \multicolumn{2}{|c|}{ Tc $60 \mathrm{~min}$} & \multicolumn{2}{|c|}{$\mathrm{Tc} 70 \mathrm{~min}$} & \multicolumn{2}{|c|}{ Tc $80 \mathrm{~min}$} \\
\hline Fv 60\% & $\mathrm{Ra}$ & $\mathrm{Rz}$ & $\mathrm{Ra}$ & $\mathrm{Rz}$ & $\mathrm{Ra}$ & $\mathrm{Rz}$ & $\mathrm{Ra}$ & $\mathrm{Rz}$ & $\mathrm{Ra}$ & $\mathrm{Rz}$ & $\mathrm{Ra}$ & $\mathrm{Rz}$ \\
\hline 1 & 5.39 & 31.32 & 7.52 & 40.35 & 8.50 & 68.22 & 7.68 & 48.17 & 8.69 & 53.60 & 8.58 & 39.95 \\
\hline 2 & 4.07 & 29.71 & 5.43 & 35.71 & 6.46 & 40.25 & 7.34 & 37.52 & 5.33 & 32.43 & 6.04 & 38.37 \\
\hline 3 & 6.42 & 40.09 & 5.60 & 37.21 & 6.28 & 38.12 & 8.31 & 46.92 & 6.16 & 32.84 & 9.73 & 64.05 \\
\hline 4 & 2.81 & 17.63 & 4.21 & 26.13 & 7.42 & 46.61 & 7.73 & 45.66 & 6.32 & 35.61 & 10.17 & 51.11 \\
\hline 5 & 4.17 & 23.67 & 4.80 & 29.93 & 8.64 & 58.38 & 6.07 & 39.55 & 6.54 & 34.49 & 5.96 & 36.63 \\
\hline 6 & 4.60 & 26.03 & 5.98 & 35.13 & 7.61 & 46.42 & 7.74 & 45.84 & 4.47 & 26.23 & 8.92 & 55.02 \\
\hline 7 & 5.53 & 32.13 & 6.68 & 42.33 & 5.66 & 36.51 & 4.88 & 29.97 & 8.87 & 57.39 & 7.86 & 42.45 \\
\hline 8 & 5.07 & 35.69 & 7.94 & 43.61 & 6.78 & 33.62 & 7.89 & 43.64 & 8.27 & 47.43 & 8.34 & 46.53 \\
\hline 9 & 3.79 & 26.53 & 4.03 & 24.27 & 5.10 & 30.25 & 7.15 & 37.67 & 7.98 & 49.21 & 8.48 & 50.40 \\
\hline 10 & 4.33 & 32.60 & 6.37 & 39.01 & 5.56 & 31.49 & 8.34 & 52.29 & 7.73 & 42.66 & 8.55 & 47.65 \\
\hline 11 & 6.45 & 39.48 & 7.23 & 37.81 & 4.99 & 27.21 & 5.41 & 31.50 & 9.24 & 55.25 & 8.83 & 48.40 \\
\hline 12 & 7.28 & 40.67 & 5.92 & 36.73 & 4.32 & 24.85 & 6.69 & 44.09 & 7.71 & 44.74 & 6.80 & 39.95 \\
\hline 13 & 6.34 & 34.62 & 5.56 & 34.19 & 6.54 & 38.85 & 6.37 & 41.42 & 5.39 & 37.48 & 9.29 & 58.18 \\
\hline 14 & 5.36 & 31.95 & 4.01 & 28.86 & 6.03 & 36.49 & 5.16 & 32.70 & 6.33 & 37.86 & 5.65 & 41.82 \\
\hline 15 & 4.96 & 28.46 & 5.99 & 43.94 & 5.30 & 33.54 & 7.40 & 45.47 & 7.90 & 48.22 & 6.65 & 38.47 \\
\hline media & 5.10 & 31.37 & 5.82 & 35.68 & 6.35 & 39.39 & 6.94 & 41.49 & 7.13 & 42.36 & 7.99 & 46.60 \\
\hline $\mathrm{s}$ & 1.19 & 6.39 & 1.22 & 6.09 & 1.27 & 11.61 & 1.13 & 6.54 & 1.44 & 9.38 & 1.43 & 8.06 \\
\hline E05 & Tc & $\min$ & Tc 3 & $\min$ & Tc 5 & $\min$ & Tc 6 & $\min$ & Tc 7 & $\min$ & Tc 80 & $\min$ \\
\hline Fv 60\% & $\mathrm{Ra}$ & $\mathrm{Rz}$ & $\mathrm{Ra}$ & $\mathrm{Rz}$ & $\mathrm{Ra}$ & $\mathrm{Rz}$ & $\mathrm{Ra}$ & $\mathrm{Rz}$ & $\mathrm{Ra}$ & $\mathrm{Rz}$ & $\mathrm{Ra}$ & $\mathrm{Rz}$ \\
\hline 1 & 3.41 & 23.98 & 3.88 & 22.91 & 3.50 & 19.10 & 3.84 & 22.26 & 3.55 & 25.71 & 3.71 & 18.71 \\
\hline 2 & 2.68 & 20.26 & 3.57 & 19.69 & 2.99 & 20.07 & 3.55 & 19.63 & 3.60 & 22.76 & 2.59 & 15.38 \\
\hline 3 & 2.50 & 16.28 & 2.04 & 12.36 & 3.12 & 23.16 & 4.01 & 20.11 & 4.50 & 29.87 & 3.87 & 23.06 \\
\hline 4 & 2.48 & 15.97 & 3.06 & 19.33 & 2.28 & 17.20 & 2.55 & 16.74 & 2.52 & 14.00 & 3.28 & 19.67 \\
\hline 5 & 2.52 & 16.18 & 2.95 & 18.65 & 2.64 & 17.78 & 2.47 & 14.25 & 4.20 & 25.82 & 3.63 & 24.17 \\
\hline 6 & 2.14 & 14.91 & 3.87 & 22.07 & 2.39 & 16.52 & 2.63 & 19.24 & 3.64 & 25.23 & 2.51 & 14.73 \\
\hline
\end{tabular}




\begin{tabular}{|c|c|c|c|c|c|c|c|c|c|c|c|c|}
\hline E05 & \multicolumn{2}{|c|}{ Tc $5 \mathrm{~min}$} & \multicolumn{2}{|c|}{ Tc $30 \mathrm{~min}$} & \multicolumn{2}{|c|}{ Tc $50 \mathrm{~min}$} & \multicolumn{2}{|c|}{ Tc $60 \mathrm{~min}$} & \multicolumn{2}{|c|}{ Tc $70 \mathrm{~min}$} & \multicolumn{2}{|c|}{ Tc $80 \mathrm{~min}$} \\
\hline Fv 60\% & $\mathrm{Ra}$ & $\mathrm{Rz}$ & $\mathrm{Ra}$ & $\mathrm{Rz}$ & $\mathrm{Ra}$ & $\mathrm{Rz}$ & $\mathrm{Ra}$ & $\mathrm{Rz}$ & $\mathrm{Ra}$ & $\mathrm{Rz}$ & $\mathrm{Ra}$ & $\mathrm{Rz}$ \\
\hline 7 & 2.48 & 14.11 & 2.66 & 16.66 & 2.29 & 14.68 & 2.45 & 15.41 & 3.29 & 19.92 & 3.57 & 22.73 \\
\hline 8 & 2.21 & 13.24 & 2.26 & 15.64 & 2.72 & 17.55 & 3.12 & 18.29 & 2.21 & 12.36 & 3.70 & 21.12 \\
\hline 9 & 2.31 & 14.07 & 2.33 & 14.68 & 2.36 & 13.04 & 3.71 & 28.55 & 2.82 & 18.09 & 2.80 & 18.21 \\
\hline 10 & 2.01 & 13.46 & 2.88 & 18.87 & 2.31 & 14.04 & 2.19 & 14.33 & 3.24 & 22.85 & 2.42 & 15.24 \\
\hline 11 & 1.91 & 11.14 & 1.71 & 11.68 & 2.32 & 17.07 & 2.57 & 18.36 & 2.68 & 17.95 & 2.91 & 16.06 \\
\hline 12 & 2.48 & 14.26 & 2.90 & 17.84 & 3.20 & 20.81 & 2.72 & 17.46 & 3.23 & 24.19 & 3.58 & 27.25 \\
\hline 13 & 2.82 & 15.10 & 2.77 & 16.41 & 2.22 & 15.57 & 2.66 & 16.91 & 3.42 & 22.64 & 2.97 & 18.68 \\
\hline 14 & 2.41 & 15.58 & 1.87 & 11.43 & 2.64 & 17.91 & 2.47 & 17.05 & 3.58 & 26.12 & 4.02 & 25.71 \\
\hline 15 & 2.10 & 14.89 & 2.40 & 15.71 & 2.56 & 15.33 & 2.89 & 16.26 & 3.00 & 22.83 & 4.52 & 30.34 \\
\hline media & 2.43 & 15.56 & 2.74 & 16.93 & 2.64 & 17.32 & 2.92 & 18.32 & 3.30 & 22.02 & 3.34 & 20.74 \\
\hline $\mathrm{s}$ & 0.37 & 3.05 & 0.67 & 3.49 & 0.40 & 2.69 & 0.58 & 3.57 & 0.60 & 4.76 & 0.62 & 4.73 \\
\hline E07 & \multicolumn{2}{|c|}{ Tc 5 min } & \multicolumn{2}{|c|}{ Tc $30 \mathrm{~min}$} & \multicolumn{2}{|c|}{ Tc $50 \mathrm{~min}$} & \multicolumn{2}{|c|}{ Tc $60 \mathrm{~min}$} & \multicolumn{2}{|c|}{ Tc $70 \mathrm{~min}$} & \multicolumn{2}{|c|}{ Tc $80 \mathrm{~min}$} \\
\hline Fv 60\% & $\mathrm{Ra}$ & $\mathrm{Rz}$ & $\mathrm{Ra}$ & $\mathrm{Rz}$ & $\mathrm{Ra}$ & $\mathrm{Rz}$ & $\mathrm{Ra}$ & $\mathrm{Rz}$ & $\mathrm{Ra}$ & $\mathrm{Rz}$ & $\mathrm{Ra}$ & $\mathrm{Rz}$ \\
\hline 1 & 5.69 & 33.07 & 6.14 & 43.62 & 6.31 & 38.52 & 3.26 & 22.68 & 4.99 & 37.89 & 5.44 & 32.00 \\
\hline 2 & 4.35 & 25.42 & 4.82 & 28.99 & 3.63 & 26.14 & 6.52 & 39.97 & 5.80 & 32.91 & 7.30 & 37.74 \\
\hline 3 & 1.49 & 10.99 & 6.33 & 44.22 & 4.23 & 29.39 & 5.78 & 33.14 & 9.01 & 51.17 & 9.36 & 52.03 \\
\hline 4 & 6.45 & 37.77 & 5.62 & 32.39 & 7.11 & 42.41 & 7.22 & 59.76 & 7.17 & 42.12 & 5.33 & 31.87 \\
\hline 5 & 3.18 & 25.00 & 3.16 & 25.47 & 6.12 & 35.07 & 5.79 & 34.07 & 7.21 & 43.14 & 7.01 & 40.38 \\
\hline 6 & 3.90 & 23.11 & 3.51 & 24.75 & 7.12 & 44.42 & 4.96 & 30.27 & 5.58 & 35.95 & 9.78 & 54.12 \\
\hline 7 & 3.52 & 24.44 & 5.94 & 36.14 & 4.90 & 44.50 & 5.22 & 32.03 & 7.46 & 41.91 & 5.52 & 37.45 \\
\hline 8 & 6.31 & 41.50 & 6.09 & 43.46 & 6.45 & 36.93 & 7.36 & 37.38 & 5.89 & 30.22 & 5.49 & 32.99 \\
\hline 9 & 3.67 & 19.25 & 4.28 & 25.44 & 3.06 & 19.55 & 5.93 & 31.87 & 5.41 & 35.38 & 6.65 & 39.01 \\
\hline 10 & 4.64 & 36.26 & 2.90 & 24.43 & 5.61 & 36.06 & 8.80 & 48.15 & 8.15 & 55.00 & 7.13 & 42.05 \\
\hline 11 & 6.00 & 36.36 & 7.47 & 53.56 & 6.24 & 41.15 & 8.05 & 46.58 & 4.11 & 26.65 & 7.67 & 50.93 \\
\hline 12 & 2.06 & 15.83 & 4.56 & 29.69 & 6.74 & 42.01 & 5.96 & 34.81 & 8.44 & 45.54 & 7.06 & 47.56 \\
\hline 13 & 5.05 & 33.13 & 6.28 & 41.64 & 4.30 & 28.14 & 6.96 & 45.35 & 6.17 & 38.72 & 7.41 & 48.24 \\
\hline 14 & 6.44 & 40.30 & 6.95 & 51.60 & 7.69 & 49.85 & 5.51 & 38.68 & 8.61 & 51.68 & 6.63 & 42.99 \\
\hline 15 & 4.84 & 35.71 & 5.59 & 32.74 & 4.00 & 24.93 & 4.78 & 32.12 & 7.29 & 46.02 & 7.51 & 43.38 \\
\hline media & 4.51 & 29.21 & 5.31 & 35.88 & 5.57 & 35.94 & 6.14 & 37.79 & 6.75 & 40.95 & 7.02 & 42.18 \\
\hline $\mathrm{s}$ & 1.55 & 9.35 & 1.38 & 9.86 & 1.44 & 8.63 & 1.40 & 9.10 & 1.46 & 8.13 & 1.32 & 7.24 \\
\hline E08 & \multicolumn{2}{|c|}{ Tc 5 min } & \multicolumn{2}{|c|}{ Tc $30 \mathrm{~min}$} & \multicolumn{2}{|c|}{ Tc $50 \mathrm{~min}$} & \multicolumn{2}{|c|}{ Tc $60 \mathrm{~min}$} & \multicolumn{2}{|c|}{ Tc $70 \mathrm{~min}$} & \multicolumn{2}{|c|}{ Tc $80 \mathrm{~min}$} \\
\hline Fv $60 \%$ & $\mathrm{Ra}$ & $\mathrm{Rz}$ & $\mathrm{Ra}$ & $\mathrm{Rz}$ & $\mathrm{Ra}$ & $\mathrm{Rz}$ & $\mathrm{Ra}$ & $\mathrm{Rz}$ & $\mathrm{Ra}$ & $\mathrm{Rz}$ & $\mathrm{Ra}$ & $\mathrm{Rz}$ \\
\hline 1 & 5.31 & 30.23 & 6.48 & 37.91 & 7.33 & 43.35 & 8.43 & 49.16 & 11.38 & 61.30 & 7.10 & 41.04 \\
\hline 2 & 6.55 & 36.65 & 6.15 & 34.84 & 4.33 & 28.77 & 8.04 & 49.14 & 9.72 & 46.89 & 7.46 & 46.39 \\
\hline 3 & 5.43 & 31.56 & 6.01 & 36.25 & 7.22 & 43.52 & 5.31 & 25.33 & 5.89 & 37.77 & 7.06 & 45.11 \\
\hline 4 & 8.34 & 44.99 & 7.38 & 36.29 & 6.20 & 37.61 & 5.68 & 22.18 & 7.92 & 45.52 & 9.32 & 51.73 \\
\hline 5 & 5.62 & 33.03 & 8.21 & 52.55 & 5.70 & 37.38 & 8.53 & 50.30 & 7.05 & 42.18 & 11.14 & 59.63 \\
\hline 6 & 5.94 & 41.34 & 5.16 & 31.49 & 7.81 & 51.69 & 5.08 & 29.22 & 5.97 & 32.53 & 9.85 & 59.98 \\
\hline 7 & 8.51 & 48.29 & 6.58 & 34.17 & 5.50 & 36.24 & 8.01 & 53.09 & 9.97 & 55.69 & 7.17 & 47.32 \\
\hline 8 & 6.61 & 37.85 & 7.35 & 40.52 & 9.24 & 53.00 & 11.25 & 53.29 & 13.12 & 62.96 & 10.06 & 52.28 \\
\hline 9 & 4.14 & 24.48 & 7.22 & 46.27 & 9.51 & 54.28 & 8.99 & 46.95 & 7.30 & 39.76 & 12.12 & 56.21 \\
\hline 10 & 4.72 & 29.61 & 6.70 & 37.29 & 6.50 & 36.33 & 6.57 & 38.93 & 9.50 & 49.37 & 6.89 & 40.09 \\
\hline 11 & 4.32 & 24.75 & 5.67 & 33.64 & 9.48 & 51.77 & 7.93 & 42.55 & 6.45 & 36.88 & 10.13 & 56.95 \\
\hline 12 & 5.06 & 28.91 & 6.70 & 35.43 & 5.27 & 32.77 & 8.92 & 53.00 & 8.47 & 52.45 & 7.42 & 45.39 \\
\hline 13 & 5.17 & 37.77 & 7.41 & 40.50 & 6.62 & 39.94 & 8.85 & 47.29 & 8.17 & 49.88 & 8.02 & 50.16 \\
\hline 14 & 8.24 & 45.65 & 7.30 & 41.95 & 5.27 & 32.40 & 9.31 & 54.49 & 11.02 & 58.17 & 8.84 & 53.86 \\
\hline
\end{tabular}


ANEXOS

\begin{tabular}{|c|c|c|c|c|c|c|c|c|c|c|c|c|}
\hline E08 & \multicolumn{2}{|c|}{ Tc $5 \mathrm{~min}$} & \multicolumn{2}{|c|}{ Tc $30 \mathrm{~min}$} & \multicolumn{2}{|c|}{ Tc $50 \mathrm{~min}$} & \multicolumn{2}{|c|}{ Tc $60 \mathrm{~min}$} & \multicolumn{2}{|c|}{ Tc $70 \mathrm{~min}$} & \multicolumn{2}{|c|}{ Tc $80 \mathrm{~min}$} \\
\hline Fv 60\% & $\mathrm{Ra}$ & $\mathrm{Rz}$ & $\mathrm{Ra}$ & $\mathrm{Rz}$ & $\mathrm{Ra}$ & $\mathrm{Rz}$ & $\mathrm{Ra}$ & $\mathrm{Rz}$ & $\mathrm{Ra}$ & $\mathrm{Rz}$ & $\mathrm{Ra}$ & $\mathrm{Rz}$ \\
\hline 15 & 6.32 & 35.63 & 7.10 & 39.19 & 7.82 & 47.93 & 5.88 & 36.15 & 7.67 & 44.64 & 8.78 & 47.53 \\
\hline media & 6.02 & 35.38 & 6.76 & 38.55 & 6.92 & 41.80 & 7.79 & 43.40 & 8.64 & 47.73 & 8.76 & 50.24 \\
\hline $\mathrm{s}$ & 1.41 & 7.40 & 0.79 & 5.37 & 1.62 & 8.29 & 1.74 & 10.69 & 2.11 & 9.16 & 1.64 & 6.28 \\
\hline E11 & \multicolumn{2}{|c|}{ Tc 5 min } & \multicolumn{2}{|c|}{ Tc $30 \mathrm{~min}$} & \multicolumn{2}{|c|}{ Tc $50 \mathrm{~min}$} & \multicolumn{2}{|c|}{ Tc $60 \mathrm{~min}$} & \multicolumn{2}{|c|}{ Tc $70 \mathrm{~min}$} & \multicolumn{2}{|c|}{ Tc $80 \mathrm{~min}$} \\
\hline Fv 60\% & $\mathrm{Ra}$ & $\mathrm{Rz}$ & $\mathrm{Ra}$ & $\mathrm{Rz}$ & $\mathrm{Ra}$ & $\mathrm{Rz}$ & $\mathrm{Ra}$ & $\mathrm{Rz}$ & $\mathrm{Ra}$ & $\mathrm{Rz}$ & $\mathrm{Ra}$ & $\mathrm{Rz}$ \\
\hline 1 & 2.51 & 16.57 & 3.24 & 9.19 & 3.91 & 28.22 & 3.57 & 21.98 & 4.53 & 29.10 & 5.05 & 26.05 \\
\hline 2 & 2.68 & 16.84 & 3.23 & 20.10 & 3.16 & 19.59 & 3.23 & 18.61 & 4.22 & 29.13 & 3.44 & 22.34 \\
\hline 3 & 2.15 & 13.13 & 3.52 & 25.58 & 3.25 & 19.94 & 3.14 & 20.23 & 3.75 & 24.26 & 3.72 & 25.04 \\
\hline 4 & 2.30 & 15.74 & 2.58 & 17.43 & 3.30 & 21.44 & 4.65 & 26.59 & 3.52 & 22.04 & 4.99 & 28.45 \\
\hline 5 & 2.49 & 15.63 & 2.68 & 16.72 & 4.30 & 27.46 & 3.94 & 21.70 & 4.85 & 28.50 & 3.86 & 25.29 \\
\hline 6 & 3.65 & 28.13 & 2.79 & 18.90 & 3.76 & 26.13 & 3.42 & 19.07 & 3.82 & 21.37 & 4.16 & 25.63 \\
\hline 7 & 2.35 & 14.04 & 3.86 & 25.02 & 3.67 & 24.22 & 3.15 & 18.23 & 3.54 & 24.42 & 3.51 & 21.67 \\
\hline 8 & 2.66 & 15.73 & 3.53 & 19.59 & 2.70 & 15.97 & 3.49 & 21.46 & 3.37 & 22.84 & 3.88 & 21.46 \\
\hline 9 & 1.63 & 12.14 & 2.87 & 16.52 & 3.67 & 25.09 & 3.34 & 19.95 & 4.78 & 37.67 & 3.55 & 22.83 \\
\hline 10 & 2.26 & 13.58 & 3.13 & 19.93 & 2.59 & 16.72 & 3.96 & 21.04 & 3.45 & 22.48 & 3.70 & 23.73 \\
\hline 11 & 3.64 & 26.20 & 3.97 & 26.62 & 3.59 & 24.21 & 3.94 & 21.11 & 3.68 & 24.21 & 4.31 & 29.91 \\
\hline 12 & 2.54 & 15.53 & 2.70 & 14.54 & 5.21 & 32.12 & 3.16 & 19.44 & 3.39 & 28.83 & 3.81 & 24.04 \\
\hline 13 & 3.26 & 31.45 & 4.19 & 24.96 & 3.76 & 26.13 & 3.32 & 21.52 & 3.01 & 19.25 & 4.23 & 30.07 \\
\hline 14 & 2.06 & 11.00 & 3.97 & 25.36 & 3.12 & 18.75 & 4.03 & 24.09 & 3.15 & 18.68 & 4.11 & 26.91 \\
\hline 15 & 2.23 & 13.46 & 2.39 & 13.83 & 3.78 & 23.91 & 4.42 & 26.16 & 3.82 & 22.76 & 4.88 & 28.52 \\
\hline media & 2.56 & 17.28 & 3.24 & 19.62 & 3.58 & 23.33 & 3.65 & 21.41 & 3.79 & 25.04 & 4.08 & 25.46 \\
\hline $\mathrm{S}$ & 0.57 & 6.16 & 0.58 & 5.12 & 0.64 & 4.53 & 0.48 & 2.51 & 0.56 & 4.88 & 0.53 & 2.85 \\
\hline E12 & \multicolumn{2}{|c|}{ Tc 5 min } & \multicolumn{2}{|c|}{ Tc $30 \mathrm{~min}$} & \multicolumn{2}{|c|}{ Tc $50 \mathrm{~min}$} & \multicolumn{2}{|c|}{ Tc $60 \mathrm{~min}$} & \multicolumn{2}{|c|}{ Tc $70 \mathrm{~min}$} & \multicolumn{2}{|c|}{ Tc $80 \mathrm{~min}$} \\
\hline Fv 60\% & $\mathrm{Ra}$ & $\mathrm{Rz}$ & $\mathrm{Ra}$ & $\mathrm{Rz}$ & $\mathrm{Ra}$ & $\mathrm{Rz}$ & $\mathrm{Ra}$ & $\mathrm{Rz}$ & $\mathrm{Ra}$ & $\mathrm{Rz}$ & $\mathrm{Ra}$ & $\mathrm{Rz}$ \\
\hline $\begin{array}{l}1 \\
\end{array}$ & 2.69 & 17.50 & 2.60 & 15.65 & 3.22 & 20.41 & 4.56 & 29.92 & 3.70 & 20.56 & 2.95 & 18.54 \\
\hline 2 & 2.85 & 18.00 & 2.87 & 18.24 & 3.82 & 22.76 & 3.12 & 16.53 & 3.34 & 19.81 & 3.80 & 20.79 \\
\hline 3 & 3.53 & 19.63 & 2.75 & 17.06 & 3.20 & 22.01 & 2.91 & 16.35 & 2.90 & 17.01 & 4.19 & 27.91 \\
\hline 4 & 2.34 & 14.91 & 2.38 & 14.53 & 3.12 & 17.27 & 3.49 & 23.36 & 3.88 & 23.40 & 3.82 & 25.98 \\
\hline 5 & 2.80 & 17.29 & 2.71 & 16.23 & 3.19 & 19.60 & 4.56 & 29.72 & 3.15 & 20.45 & 4.96 & 33.61 \\
\hline 6 & 2.46 & 15.55 & 3.50 & 18.80 & 2.61 & 17.01 & 3.02 & 18.40 & 2.99 & 16.62 & 2.52 & 16.19 \\
\hline 7 & 3.19 & 20.04 & 2.89 & 18.06 & 3.34 & 20.85 & 2.33 & 15.67 & 3.00 & 17.26 & 2.39 & 15.49 \\
\hline 8 & 3.23 & 21.22 & 3.04 & 20.50 & 2.24 & 14.49 & 3.33 & 22.33 & 2.67 & 17.09 & 2.90 & 18.34 \\
\hline 9 & 2.38 & 14.97 & 3.59 & 22.85 & 3.20 & 20.40 & 2.94 & 15.16 & 3.26 & 22.31 & 3.26 & 19.99 \\
\hline 10 & 1.96 & 12.68 & 3.64 & 22.97 & 3.22 & 19.14 & 3.40 & 21.69 & 2.94 & 18.97 & 3.49 & 20.80 \\
\hline 11 & 2.69 & 19.76 & 3.04 & 21.52 & 3.06 & 21.86 & 3.11 & 17.26 & 3.04 & 16.88 & 3.19 & 18.66 \\
\hline 12 & 3.00 & 20.85 & 2.38 & 13.42 & 2.09 & 14.66 & 2.79 & 13.66 & 3.10 & 20.70 & 4.88 & 25.38 \\
\hline 13 & 2.74 & 18.16 & 2.38 & 14.59 & 2.58 & 15.46 & 2.77 & 16.98 & 4.36 & 29.81 & 3.32 & 19.22 \\
\hline 14 & 3.67 & 19.72 & 2.86 & 17.90 & 2.91 & 16.81 & 3.05 & 20.11 & 3.79 & 23.09 & 4.41 & 27.86 \\
\hline 15 & 3.54 & 20.27 & 3.17 & 18.99 & 2.96 & 18.72 & 3.74 & 23.21 & 3.94 & 29.43 & 3.96 & 20.90 \\
\hline media & 2.87 & 18.04 & 2.92 & 18.09 & 2.98 & 18.76 & 3.27 & 20.02 & 3.34 & 20.89 & 3.60 & 21.98 \\
\hline $\mathrm{S}$ & 0.49 & 2.54 & 0.42 & 2.96 & 0.44 & 2.70 & 0.62 & 5.00 & 0.48 & 4.21 & 0.79 & 5.07 \\
\hline E14 & & $\min$ & Tc 3 & $\min$ & & & E14 & & $5 \mathrm{mi}$ & & Tc 30 & $\min$ \\
\hline Fv $60 \%$ & $\mathrm{Ra}$ & $\mathrm{Rz}$ & $\mathrm{Ra}$ & $\mathrm{Rz}$ & & & Fv 60 & $\mathrm{Ra}$ & & $z$ & $\mathrm{Ra}$ & $\mathrm{Rz}$ \\
\hline 1 & 3.39 & 16.97 & 5.97 & 33.28 & & & 9 & 5.90 & & 30 & 4.98 & 29.58 \\
\hline 2 & 4.16 & 29.01 & 7.00 & 38.04 & & & 10 & 4.09 & & .18 & 3.33 & 21.96 \\
\hline
\end{tabular}




\begin{tabular}{ccccc}
\hline E14 & \multicolumn{2}{c}{ Tc 5 min } & \multicolumn{2}{c}{ Tc 30 min } \\
\hline Fv 60\% & $\mathrm{Ra}$ & $\mathrm{Rz}$ & $\mathrm{Ra}$ & $\mathrm{Rz}$ \\
\hline 3 & 4.10 & 22.12 & 4.89 & 27.96 \\
\hline 4 & 4.57 & 29.13 & 6.78 & 36.26 \\
\hline 5 & 3.67 & 20.50 & 5.15 & 31.22 \\
\hline 6 & 4.11 & 27.40 & 4.12 & 25.26 \\
\hline 7 & 5.78 & 36.98 & 2.96 & 17.81 \\
\hline 8 & 4.99 & 29.82 & 5.62 & 35.37 \\
\hline
\end{tabular}

\begin{tabular}{ccccc}
\hline E14 & \multicolumn{2}{c}{ Tc 5 min } & \multicolumn{2}{c}{ Tc 30 min } \\
\hline Fv 60\% & $\mathrm{Ra}$ & $\mathrm{Rz}$ & $\mathrm{Ra}$ & $\mathrm{Rz}$ \\
\hline 11 & 2.90 & 16.72 & 3.89 & 25.78 \\
\hline 12 & 3.70 & 20.34 & 4.94 & 32.68 \\
\hline 13 & 3.12 & 17.79 & 5.44 & 32.25 \\
\hline 14 & 4.88 & 34.37 & 5.13 & 26.42 \\
\hline 15 & 2.93 & 15.24 & 3.95 & 24.85 \\
\hline media & 4.15 & 25.19 & 4.94 & 29.25 \\
\hline $\mathrm{s}$ & 0.94 & 7.32 & 1.16 & 5.64 \\
\hline
\end{tabular}

\begin{tabular}{ccccccccccccc}
\hline E16 & \multicolumn{1}{c}{ Tc $5 \mathrm{~min}$} & \multicolumn{2}{c}{$\mathrm{Tc} 30 \mathrm{~min}$} & \multicolumn{2}{c}{$\mathrm{Tc} 50 \mathrm{~min}$} & \multicolumn{2}{c}{$\mathrm{Tc} 60 \mathrm{~min}$} & \multicolumn{2}{c}{ Tc $70 \mathrm{~min}$} & \multicolumn{2}{c}{ Tc $80 \mathrm{~min}$} \\
\hline Fv 60\% & $\mathrm{Ra}$ & $\mathrm{Rz}$ & $\mathrm{Ra}$ & $\mathrm{Rz}$ & $\mathrm{Ra}$ & $\mathrm{Rz}$ & $\mathrm{Ra}$ & $\mathrm{Rz}$ & $\mathrm{Ra}$ & $\mathrm{Rz}$ & $\mathrm{Ra}$ & $\mathrm{Rz}$ \\
\hline 1 & 4.91 & 30.01 & 6.15 & 35.91 & 5.00 & 29.91 & 5.55 & 33.56 & 8.54 & 48.29 & 5.39 & 31.40 \\
\hline 2 & 5.59 & 31.17 & 6.67 & 43.88 & 6.37 & 42.67 & 7.50 & 44.41 & 5.69 & 31.53 & 5.68 & 33.89 \\
\hline 3 & 7.31 & 41.55 & 5.52 & 33.65 & 6.72 & 43.46 & 4.69 & 30.88 & 7.35 & 49.00 & 7.07 & 44.78 \\
\hline 4 & 4.75 & 29.41 & 5.73 & 32.14 & 7.83 & 47.00 & 5.67 & 32.36 & 5.19 & 36.14 & 6.71 & 33.47 \\
\hline 5 & 6.73 & 39.34 & 6.74 & 40.42 & 6.04 & 32.94 & 6.63 & 38.63 & 7.20 & 40.61 & 7.00 & 43.60 \\
\hline 6 & 6.12 & 41.31 & 6.40 & 38.91 & 7.25 & 46.22 & 6.14 & 33.40 & 4.79 & 28.11 & 7.45 & 38.93 \\
\hline 7 & 6.91 & 44.35 & 5.56 & 32.14 & 5.25 & 29.86 & 5.44 & 29.44 & 5.35 & 32.24 & 7.05 & 39.95 \\
\hline 8 & 3.68 & 26.47 & 7.16 & 50.59 & 7.02 & 40.47 & 7.30 & 46.56 & 6.07 & 34.71 & 7.69 & 40.47 \\
\hline 9 & 4.24 & 28.27 & 6.63 & 43.39 & 4.24 & 26.06 & 8.08 & 46.83 & 5.39 & 34.78 & 8.31 & 49.05 \\
\hline 10 & 6.02 & 31.33 & 5.94 & 39.44 & 5.32 & 34.80 & 4.52 & 29.41 & 7.84 & 41.34 & 5.78 & 32.38 \\
\hline 11 & 5.42 & 36.07 & 4.92 & 32.32 & 6.35 & 37.46 & 6.90 & 44.66 & 7.39 & 38.86 & 7.84 & 41.75 \\
\hline 12 & 6.08 & 31.32 & 5.23 & 34.70 & 6.50 & 44.23 & 6.95 & 43.11 & 6.42 & 40.61 & 6.30 & 40.55 \\
\hline 13 & 4.55 & 28.86 & 6.16 & 31.85 & 7.05 & 41.87 & 8.01 & 44.80 & 7.51 & 48.12 & 8.47 & 49.32 \\
\hline 14 & 5.58 & 30.39 & 5.46 & 36.68 & 6.81 & 39.79 & 6.50 & 37.15 & 8.53 & 38.71 & 7.67 & 40.82 \\
\hline 15 & 4.90 & 30.12 & 6.77 & 37.00 & 5.19 & 31.73 & 7.67 & 44.06 & 6.47 & 38.77 & 6.55 & 39.88 \\
\hline media & 5.52 & 33.33 & 6.07 & 37.53 & 6.20 & 37.90 & 6.50 & 38.62 & 6.65 & 38.79 & 7.00 & 40.02 \\
\hline $\mathrm{S}$ & 1.03 & 5.65 & 0.66 & 5.37 & 1.00 & 6.62 & 1.14 & 6.61 & 1.22 & 6.23 & 0.94 & 5.49 \\
\hline
\end{tabular}


Puntos medidos para el experimento 14

\begin{tabular}{ccccccccccc}
\hline E14 & \multicolumn{2}{c}{ Tc $1.1 \mathrm{~min}$} & \multicolumn{2}{c}{$\mathrm{Tc} 7.5 \mathrm{~min}$} & \multicolumn{2}{c}{$\mathrm{Tc} 10 \mathrm{~min}$} & \multicolumn{2}{c}{ Tc $12.5 \mathrm{~min}$} & \multicolumn{2}{c}{ Tc $15 \mathrm{~min}$} \\
\hline Fv 60\% & $\mathrm{Ra}$ & $\mathrm{Rz}$ & $\mathrm{Ra}$ & $\mathrm{Rz}$ & $\mathrm{Ra}$ & $\mathrm{Rz}$ & $\mathrm{Ra}$ & $\mathrm{Rz}$ & $\mathrm{Ra}$ & $\mathrm{Rz}$ \\
\hline 1 & 3.90 & 23.05 & 5.15 & 26.81 & 5.48 & 32.21 & 5.82 & 37.14 & 3.39 & 20.91 \\
\hline 2 & 3.70 & 24.04 & 3.14 & 18.98 & 4.73 & 25.26 & 3.59 & 21.16 & 4.22 & 23.15 \\
\hline 3 & 4.64 & 27.44 & 2.66 & 14.46 & 4.40 & 24.38 & 6.35 & 34.67 & 5.12 & 28.83 \\
\hline 4 & 5.02 & 28.38 & 3.96 & 24.46 & 3.97 & 26.16 & 3.89 & 19.58 & 5.30 & 30.33 \\
\hline 5 & 3.75 & 22.38 & 3.48 & 22.11 & 5.05 & 30.58 & 5.95 & 48.57 & 7.05 & 28.07 \\
\hline 6 & 2.74 & 18.55 & 5.11 & 27.85 & 4.35 & 24.47 & 3.05 & 16.41 & 4.18 & 24.66 \\
\hline 7 & 3.72 & 21.60 & 4.59 & 30.44 & 3.60 & 20.34 & 2.89 & 19.17 & 2.78 & 16.77 \\
\hline 8 & 4.14 & 23.78 & 3.18 & 19.02 & 3.24 & 17.59 & 4.38 & 25.97 & 4.60 & 26.61 \\
\hline 9 & 3.30 & 21.33 & 3.34 & 21.28 & 5.76 & 34.00 & 3.90 & 20.34 & 3.95 & 21.68 \\
\hline 10 & 3.15 & 18.31 & 4.22 & 25.45 & 4.61 & 29.33 & 2.81 & 17.10 & 4.07 & 26.69 \\
\hline 11 & 4.91 & 24.96 & 5.76 & 31.97 & 3.08 & 19.34 & 3.16 & 18.17 & 3.35 & 21.01 \\
\hline 12 & 3.54 & 20.59 & 3.21 & 21.81 & 3.49 & 15.56 & 4.15 & 22.99 & 4.08 & 26.02 \\
\hline 13 & 3.14 & 19.50 & 4.57 & 26.35 & 4.56 & 27.91 & 4.91 & 28.43 & 6.91 & 46.67 \\
\hline 14 & 2.59 & 14.51 & 4.29 & 25.76 & 3.57 & 12.45 & 3.47 & 23.43 & 3.27 & 21.39 \\
\hline 15 & 3.28 & 22.96 & 3.85 & 24.54 & 4.02 & 21.83 & 3.83 & 24.98 & 3.08 & 18.69 \\
\hline media & 3.70 & 22.09 & 4.03 & 24.09 & 4.26 & 24.09 & 4.14 & 25.21 & 4.36 & 25.43 \\
\hline $\mathrm{S}$ & 0.73 & 3.57 & 0.89 & 4.60 & 0.80 & 6.25 & 1.14 & 8.85 & 1.28 & 7.04 \\
\hline & & & & & & & & & &
\end{tabular}

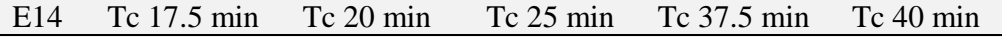

\begin{tabular}{ccccccccccc}
\hline Fv 60\% & $\mathrm{Ra}$ & $\mathrm{Rz}$ & $\mathrm{Ra}$ & $\mathrm{Rz}$ & $\mathrm{Ra}$ & $\mathrm{Rz}$ & $\mathrm{Ra}$ & $\mathrm{Rz}$ & $\mathrm{Ra}$ & $\mathrm{Rz}$ \\
\hline 1 & 4.48 & 26.93 & 7.13 & 48.81 & 4.03 & 22.32 & 7.18 & 38.72 & 3.70 & 24.91 \\
\hline 2 & 4.09 & 20.94 & 4.52 & 34.66 & 8.12 & 49.73 & 6.26 & 37.62 & 8.75 & 55.20 \\
\hline 3 & 3.68 & 25.69 & 3.59 & 19.96 & 4.01 & 26.74 & 7.63 & 46.82 & 5.79 & 31.93 \\
\hline 4 & 3.95 & 21.45 & 6.89 & 38.42 & 3.74 & 24.00 & 4.44 & 22.29 & 5.57 & 30.10 \\
\hline 5 & 7.08 & 25.81 & 5.52 & 40.57 & 5.67 & 37.08 & 4.76 & 28.16 & 4.96 & 26.34 \\
\hline 6 & 3.07 & 18.76 & 4.68 & 26.70 & 10.03 & 52.51 & 4.13 & 28.63 & 7.44 & 45.02 \\
\hline 7 & 4.24 & 27.64 & 4.25 & 30.34 & 3.08 & 17.46 & 4.37 & 30.02 & 6.21 & 34.31 \\
\hline 8 & 7.39 & 28.72 & 4.51 & 25.85 & 5.18 & 30.21 & 8.38 & 58.52 & 4.27 & 25.33 \\
\hline 9 & 3.29 & 19.72 & 2.89 & 18.07 & 5.17 & 29.35 & 4.38 & 27.21 & 4.25 & 24.58 \\
\hline 10 & 3.11 & 18.72 & 5.59 & 38.55 & 3.19 & 20.56 & 4.30 & 26.30 & 5.00 & 31.46 \\
\hline 11 & 4.41 & 27.45 & 4.57 & 29.97 & 4.57 & 28.31 & 6.51 & 38.75 & 4.33 & 27.03 \\
\hline 12 & 4.13 & 24.91 & 3.92 & 25.10 & 3.56 & 20.76 & 4.90 & 25.99 & 3.76 & 21.77 \\
\hline 13 & 4.03 & 22.66 & 5.59 & 34.36 & 3.97 & 25.07 & 5.36 & 27.97 & 5.65 & 32.58 \\
\hline 14 & 6.51 & 38.31 & 4.89 & 25.85 & 4.46 & 28.44 & 3.87 & 23.35 & 7.71 & 45.54 \\
\hline 15 & 4.31 & 25.34 & 3.11 & 19.44 & 3.72 & 24.49 & 3.59 & 23.40 & 4.12 & 26.73 \\
\hline media & 4.52 & 24.87 & 4.78 & 30.44 & 4.83 & 29.14 & 5.34 & 32.25 & 5.43 & 32.19 \\
\hline $\mathrm{s}$ & 1.37 & 5.00 & 1.22 & 8.75 & 1.91 & 10.12 & 1.49 & 10.09 & 1.53 & 9.41 \\
\hline
\end{tabular}




\section{II.2. GRÁFICOS DE RUGOSIDAD SUPERFICIAL}

Ra por volumen de fibra:
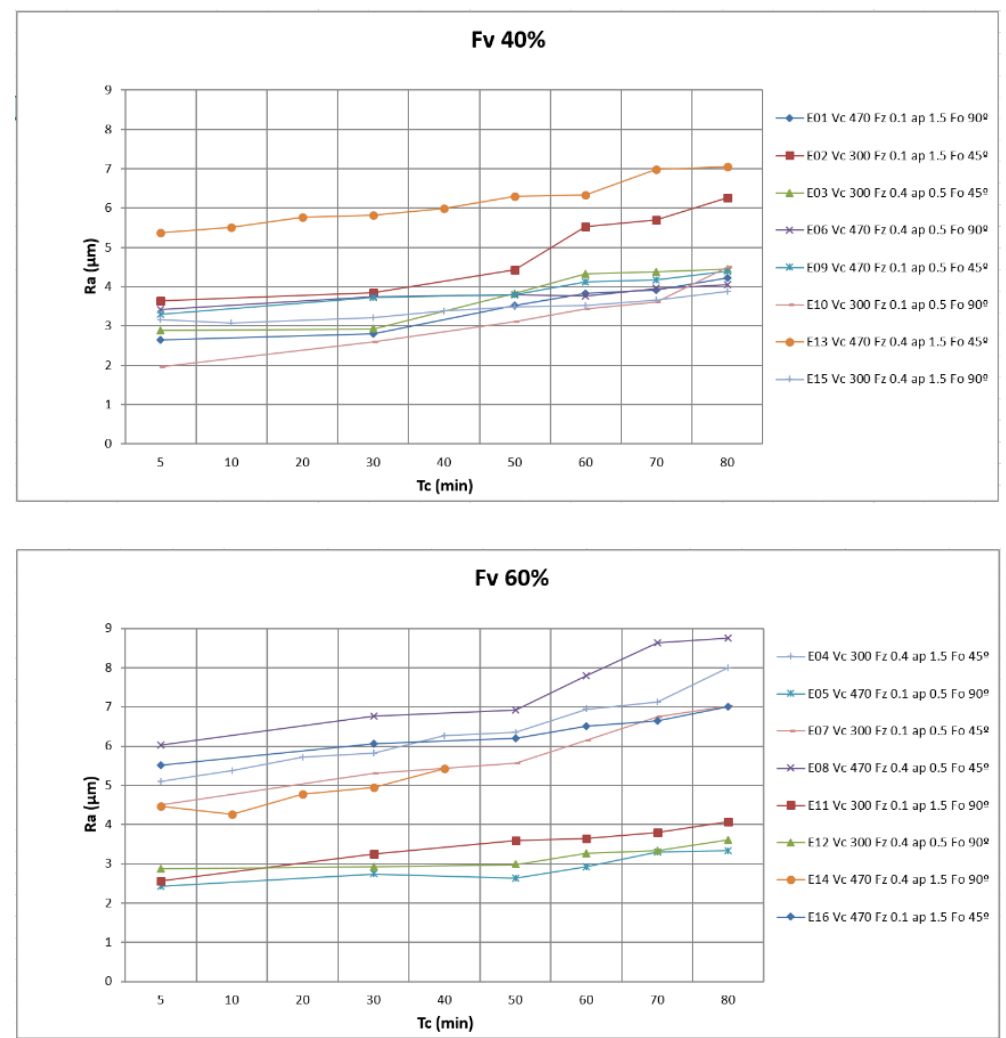

Ra por orientación de fibra:

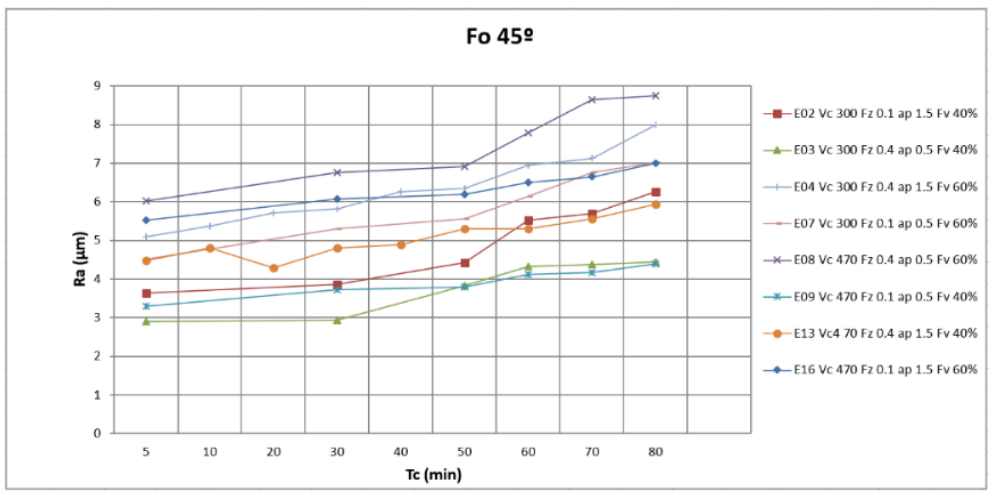




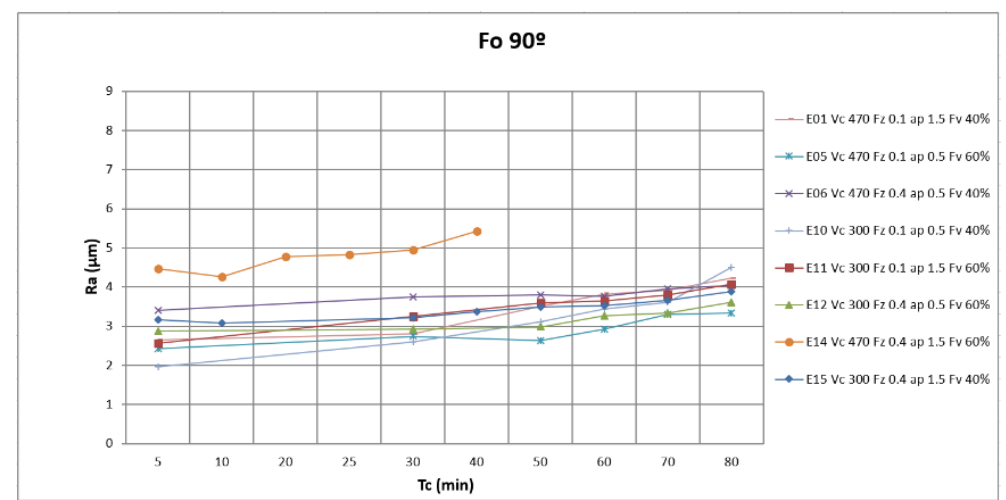




\section{II.3. MEDIDAS DE DESGASTE DE LA HERRAMIENTA}

\begin{tabular}{llcccccc}
\hline E01 & & \multicolumn{7}{c}{ Tc (min) } \\
\cline { 3 - 7 } & & 5 & 30 & 50 & 60 & 70 & 80 \\
\hline Filo 1 & $\mathrm{P} 1(\mathrm{px})$ & 17.28 & 18.23 & 19.19 & 20.98 & 21.90 & 23.43 \\
& $\mathrm{P} 2(\mathrm{px})$ & 16.72 & 17.88 & 19.05 & 20.65 & 21.57 & 22.31 \\
& $\overline{V b}(\mathrm{px})$ & 17.00 & 18.06 & 19.12 & 20.82 & 21.73 & 22.87 \\
& $\overline{V b}(\mathrm{~mm})$ & 0.061 & 0.065 & 0.069 & 0.075 & 0.078 & 0.082 \\
\hline Filo 2 & $\mathrm{P} 1(\mathrm{px})$ & 8.74 & 9.56 & 10.39 & 12.61 & 14.51 & 16.28 \\
& $\mathrm{P} 2(\mathrm{px})$ & 8.42 & 9.36 & 10.30 & 12.74 & 14.35 & 15.14 \\
& $\overline{V b}(\mathrm{px})$ & 8.58 & 9.46 & 10.34 & 12.68 & 14.43 & 15.71 \\
& $\overline{V b}(\mathrm{~mm})$ & 0.031 & 0.034 & 0.037 & 0.046 & 0.052 & 0.057 \\
\hline
\end{tabular}

\begin{tabular}{llcccccc}
\hline E02 & & \multicolumn{7}{c}{ Tc $(\mathrm{min})$} \\
\cline { 3 - 7 } & & 5 & 30 & 50 & 60 & 70 & 80 \\
\hline Filo 1 & $\mathrm{P} 1(\mathrm{px})$ & 7.41 & 10.75 & 12.82 & 14.65 & 16.91 & 17.42 \\
& $\mathrm{P} 2(\mathrm{px})$ & 6.26 & 9.53 & 10.70 & 12.81 & 15.88 & 17.10 \\
& $\overline{V b}(\mathrm{px})$ & 6.84 & 10.14 & 11.76 & 13.73 & 16.39 & 17.26 \\
& $\overline{V b}(\mathrm{~mm})$ & 0.025 & 0.037 & 0.042 & 0.049 & 0.059 & 0.062 \\
\hline Filo 2 & $\mathrm{P} 1(\mathrm{px})$ & 7.36 & 12.14 & 13.81 & 16.05 & 17.53 & 18.79 \\
& $\mathrm{P} 2(\mathrm{px})$ & 5.82 & 11.71 & 12.01 & 14.84 & 16.21 & 17.70 \\
& $\overline{V b}(\mathrm{px})$ & 6.59 & 11.93 & 12.91 & 15.45 & 16.87 & 18.24 \\
& $\overline{V b}(\mathrm{~mm})$ & 0.024 & 0.043 & 0.046 & 0.056 & 0.061 & 0.066 \\
\hline
\end{tabular}

\begin{tabular}{llcccccc}
\hline E03 & & \multicolumn{7}{c}{ Tc (min) } \\
\cline { 3 - 8 } & & 5 & 30 & 50 & 60 & 70 & 80 \\
\hline Filo 1 & $\mathrm{P} 1(\mathrm{px})$ & 7.30 & 7.82 & 9.28 & 12.84 & 14.14 & 15.50 \\
& $\mathrm{P} 2(\mathrm{px})$ & 6.98 & 8.76 & 9.59 & 12.12 & 14.72 & 15.28 \\
& $\overline{V b}(\mathrm{px})$ & 7.14 & 8.29 & 9.43 & 12.48 & 14.43 & 15.39 \\
& $\overline{V b}(\mathrm{~mm})$ & 0.026 & 0.030 & 0.034 & 0.045 & 0.052 & 0.055 \\
\hline \multirow{2}{*}{ Filo 2} & $\mathrm{P} 1(\mathrm{px})$ & 5.64 & 7.05 & 8.97 & 11.46 & 12.57 & 13.61 \\
& $\mathrm{P} 2(\mathrm{px})$ & 5.45 & 7.89 & 9.39 & 12.07 & 12.83 & 14.31 \\
& $\overline{V b}(\mathrm{px})$ & 5.54 & 7.47 & 9.18 & 11.76 & 12.70 & 13.96 \\
& $\overline{V b}(\mathrm{~mm})$ & 0.020 & 0.027 & 0.033 & 0.042 & 0.046 & 0.050 \\
\hline
\end{tabular}

\begin{tabular}{llcccccc}
\hline E04 & & \multicolumn{7}{c}{ Tc $(\mathrm{min})$} \\
\cline { 3 - 7 } & & 5 & 30 & 50 & 60 & 70 & 80 \\
\hline Filo 1 & $\mathrm{P} 1(\mathrm{px})$ & 14.40 & 19.78 & 20.65 & 22.04 & 23.93 & 24.07 \\
& $\mathrm{P} 2(\mathrm{px})$ & 15.84 & 20.26 & 20.95 & 21.27 & 22.52 & 23.42 \\
& $\overline{V b}(\mathrm{px})$ & 15.12 & 20.02 & 20.80 & 21.66 & 23.23 & 23.75 \\
& $\overline{V b}(\mathrm{~mm})$ & 0.054 & 0.072 & 0.075 & 0.078 & 0.084 & 0.085 \\
\hline Filo 2 & $\mathrm{P} 1(\mathrm{px})$ & 12.25 & 13.77 & 14.31 & 16.53 & 17.12 & 19.41 \\
& $\mathrm{P} 2(\mathrm{px})$ & 8.78 & 12.30 & 12.90 & 14.62 & 15.43 & 16.52 \\
& $\overline{V b}(\mathrm{px})$ & 10.52 & 13.04 & 13.61 & 15.58 & 16.27 & 17.97 \\
& $\overline{V b}(\mathrm{~mm})$ & 0.038 & 0.047 & 0.049 & 0.056 & 0.059 & 0.065 \\
\hline
\end{tabular}




\begin{tabular}{|c|c|c|c|c|c|c|c|}
\hline \multirow[t]{2}{*}{ E05 } & & \multicolumn{5}{|c|}{$\mathrm{Tc}(\min )$} & \multirow[b]{2}{*}{80} \\
\hline & & 5 & 30 & 50 & 60 & 70 & \\
\hline \multirow[t]{4}{*}{ Filo 1} & P1 (px) & 13.27 & 14.44 & 15.47 & 16.67 & 17.32 & 18.77 \\
\hline & $\mathrm{P} 2$ (px) & 13.78 & 14.60 & 16.03 & 18.25 & 19.77 & 21.12 \\
\hline & $\overline{V b}(\mathrm{px})$ & 13.52 & 14.52 & 15.75 & 17.46 & 18.55 & 19.95 \\
\hline & $\overline{V b}(\mathrm{~mm})$ & 0.049 & 0.052 & 0.057 & 0.063 & 0.067 & 0.072 \\
\hline \multirow[t]{4}{*}{ Filo 2} & $\mathrm{P} 1$ (px) & 6.83 & 7.48 & 7.90 & 9.90 & 10.29 & 11.73 \\
\hline & $\mathrm{P} 2(\mathrm{px})$ & 8.71 & 9.20 & 10.48 & 11.57 & 12.09 & 13.71 \\
\hline & $\overline{V b}(\mathrm{px})$ & 7.77 & 8.34 & 9.19 & 10.73 & 11.19 & 12.72 \\
\hline & $\overline{V b}(\mathrm{~mm})$ & 0.028 & 0.030 & 0.033 & 0.039 & 0.040 & 0.046 \\
\hline \multirow[t]{2}{*}{ E06 } & & \multicolumn{5}{|c|}{$\mathrm{Tc}(\min )$} & \\
\hline & & 5 & 30 & 50 & 60 & 70 & 80 \\
\hline \multirow[t]{4}{*}{ Filo 1} & P1 (px) & 24.83 & 26.74 & 28.37 & 31.04 & 33.99 & 35.30 \\
\hline & $\mathrm{P} 2(\mathrm{px})$ & 21.50 & 21.74 & 22.13 & 25.45 & 28.77 & 29.76 \\
\hline & $\overline{V b}(\mathrm{px})$ & 23.17 & 24.24 & 25.25 & 28.25 & 31.38 & 32.53 \\
\hline & $\overline{V b}(\mathrm{~mm})$ & 0.083 & 0.087 & 0.091 & 0.102 & 0.113 & 0.117 \\
\hline \multirow[t]{4}{*}{ Filo 2} & $\mathrm{P} 1$ (px) & 12.63 & 15.19 & 17.79 & 20.20 & 23.50 & 24.93 \\
\hline & $\mathrm{P} 2(\mathrm{px})$ & 13.85 & 15.11 & 15.71 & 18.44 & 20.04 & 21.70 \\
\hline & $\overline{V b}(\mathrm{px})$ & 13.24 & 15.15 & 16.75 & 19.32 & 21.77 & 23.32 \\
\hline & $\overline{V b}(\mathrm{~mm})$ & 0.048 & 0.055 & 0.060 & 0.070 & 0.078 & 0.084 \\
\hline
\end{tabular}

\begin{tabular}{|c|c|c|c|c|c|c|c|}
\hline \multirow[t]{2}{*}{ E07 } & & \multicolumn{5}{|c|}{$\mathrm{Tc}(\min )$} & \multirow[b]{2}{*}{80} \\
\hline & & 5 & 30 & 50 & 60 & 70 & \\
\hline \multirow[t]{4}{*}{ Filo 1} & $\mathrm{P} 1$ (px) & 6.36 & 7.52 & 8.75 & 11.48 & 12.78 & 13.75 \\
\hline & $\mathrm{P} 2(\mathrm{px})$ & 5.80 & 8.13 & 9.24 & 11.11 & 12.53 & 13.29 \\
\hline & $\overline{V b}(\mathrm{px})$ & 6.08 & 7.83 & 9.00 & 11.29 & 12.65 & 13.52 \\
\hline & $\overline{V b}(\mathrm{~mm})$ & 0.022 & 0.028 & 0.032 & 0.041 & 0.046 & 0.049 \\
\hline \multirow[t]{4}{*}{ Filo 2} & P1 (px) & 4.60 & 7.20 & 7.44 & 8.39 & 9.29 & 10.49 \\
\hline & $\mathrm{P} 2(\mathrm{px})$ & 5.64 & 6.96 & 7.34 & 8.72 & 9.36 & 11.44 \\
\hline & $\overline{V b}(\mathrm{px})$ & 5.12 & 7.08 & 7.39 & 8.56 & 9.32 & 10.97 \\
\hline & $\overline{V b}(\mathrm{~mm})$ & 0.018 & 0.025 & 0.027 & 0.031 & 0.034 & 0.039 \\
\hline
\end{tabular}

\begin{tabular}{llcccccc}
\hline E08 & & \multicolumn{7}{c}{ Tc (min) } \\
\cline { 3 - 7 } & & 5 & 30 & 50 & 60 & 70 & 80 \\
\hline Filo 1 & $\mathrm{P} 1(\mathrm{px})$ & 23.59 & 24.76 & 25.58 & 29.23 & 29.46 & 30.06 \\
& $\mathrm{P} 2(\mathrm{px})$ & 19.55 & 20.25 & 21.01 & 23.50 & 24.63 & 25.87 \\
& $\overline{V b}(\mathrm{px})$ & 21.57 & 22.51 & 23.30 & 26.37 & 27.05 & 27.96 \\
& $\overline{V b}(\mathrm{~mm})$ & 0.078 & 0.081 & 0.084 & 0.095 & 0.097 & 0.101 \\
\hline Filo 2 & $\mathrm{P} 1(\mathrm{px})$ & 15.22 & 16.33 & 17.69 & 20.81 & 21.47 & 22.33 \\
& $\mathrm{P} 2(\mathrm{px})$ & 14.81 & 15.23 & 15.71 & 18.86 & 19.70 & 20.08 \\
& $\overline{V b}(\mathrm{px})$ & 15.02 & 15.78 & 16.70 & 19.84 & 20.58 & 21.21 \\
& $\overline{V b}(\mathrm{~mm})$ & 0.054 & 0.057 & 0.060 & 0.071 & 0.074 & 0.076 \\
\hline
\end{tabular}




\begin{tabular}{|c|c|c|c|c|c|c|c|}
\hline \multirow[t]{2}{*}{ E09 } & & \multicolumn{5}{|c|}{$\mathrm{Tc}(\min )$} & \multirow[b]{2}{*}{80} \\
\hline & & 5 & 30 & 50 & 60 & 70 & \\
\hline \multirow[t]{4}{*}{ Filo 1} & P1 (px) & 7.37 & 7.92 & 8.79 & 10.52 & 12.36 & 13.48 \\
\hline & $\mathrm{P} 2$ (px) & 6.09 & 7.00 & 8.34 & 10.57 & 12.47 & 13.50 \\
\hline & $\overline{V b}(\mathrm{px})$ & 6.73 & 7.46 & 8.56 & 10.55 & 12.42 & 13.49 \\
\hline & $\overline{V b}(\mathrm{~mm})$ & 0.024 & 0.027 & 0.031 & 0.038 & 0.045 & 0.049 \\
\hline \multirow[t]{4}{*}{ Filo 2} & $\mathrm{P} 1$ (px) & 2.67 & 3.52 & 4.58 & 6.27 & 7.15 & 8.43 \\
\hline & $\mathrm{P} 2(\mathrm{px})$ & 2.42 & 3.63 & 5.21 & 6.84 & 7.86 & 9.44 \\
\hline & $\overline{V b}(\mathrm{px})$ & 2.55 & 3.58 & 4.90 & 6.55 & 7.51 & 8.94 \\
\hline & $\overline{V b}(\mathrm{~mm})$ & 0.009 & 0.013 & 0.018 & 0.024 & 0.027 & 0.032 \\
\hline \multirow[t]{2}{*}{ E10 } & & \multicolumn{5}{|c|}{$\mathrm{Tc}(\min )$} & \\
\hline & & 5 & 30 & 50 & 60 & 70 & 80 \\
\hline \multirow[t]{4}{*}{ Filo 1} & P1 (px) & 6.94 & 8.12 & 9.44 & 11.89 & 12.07 & 13.19 \\
\hline & $\mathrm{P} 2(\mathrm{px})$ & 7.76 & 8.50 & 11.00 & 12.46 & 13.92 & 15.84 \\
\hline & $\overline{V b}(\mathrm{px})$ & 7.35 & 8.31 & 10.22 & 12.17 & 12.99 & 14.52 \\
\hline & $\overline{V b}(\mathrm{~mm})$ & 0.026 & 0.030 & 0.037 & 0.044 & 0.047 & 0.052 \\
\hline \multirow[t]{4}{*}{ Filo 2} & $\mathrm{P} 1$ (px) & 4.87 & 5.92 & 8.28 & 9.74 & 10.92 & 12.24 \\
\hline & $\mathrm{P} 2(\mathrm{px})$ & 5.80 & 7.10 & 9.53 & 11.29 & 13.46 & 14.43 \\
\hline & $\overline{V b}(\mathrm{px})$ & 5.33 & 6.51 & 8.90 & 10.52 & 12.19 & 13.34 \\
\hline & $\overline{V b}(\mathrm{~mm})$ & 0.019 & 0.023 & 0.032 & 0.038 & 0.044 & 0.048 \\
\hline
\end{tabular}

\begin{tabular}{llcccccc}
\hline E11 & & \multicolumn{7}{c}{ Tc $(\mathrm{min})$} \\
\cline { 3 - 7 } & & 5 & 30 & 50 & 60 & 70 & 80 \\
\hline Filo 1 & $\mathrm{P} 1(\mathrm{px})$ & 9.17 & 11.19 & 13.52 & 17.06 & 19.01 & 21.45 \\
& $\mathrm{P} 2(\mathrm{px})$ & 8.77 & 10.57 & 12.66 & 15.42 & 17.59 & 19.13 \\
& $\overline{V b}(\mathrm{px})$ & 8.97 & 10.88 & 13.09 & 16.24 & 18.30 & 20.29 \\
& $\overline{V b}(\mathrm{~mm})$ & 0.032 & 0.039 & 0.047 & 0.058 & 0.066 & 0.073 \\
\hline \multirow{2}{*}{ Filo 2} & $\mathrm{P} 1(\mathrm{px})$ & 5.32 & 8.24 & 10.84 & 12.99 & 13.11 & 14.59 \\
& $\mathrm{P} 2(\mathrm{px})$ & 4.40 & 5.97 & 9.96 & 11.86 & 12.11 & 13.85 \\
& $\overline{V b}(\mathrm{px})$ & 4.86 & 7.10 & 10.40 & 12.42 & 12.61 & 14.22 \\
& $\overline{V b}(\mathrm{~mm})$ & 0.017 & 0.026 & 0.037 & 0.045 & 0.045 & 0.051 \\
\hline
\end{tabular}

\begin{tabular}{llcccccc}
\hline E12 & & \multicolumn{7}{c}{ Tc $(\mathrm{min})$} \\
\cline { 3 - 7 } & & 5 & 30 & 50 & 60 & 70 & 80 \\
\hline Filo 1 & $\mathrm{P} 1(\mathrm{px})$ & 11.81 & 13.52 & 13.94 & 15.93 & 16.94 & 17.65 \\
& $\mathrm{P} 2(\mathrm{px})$ & 10.78 & 11.99 & 12.66 & 15.19 & 16.52 & 17.21 \\
& $\overline{V b}(\mathrm{px})$ & 11.30 & 12.75 & 13.30 & 15.56 & 16.73 & 17.43 \\
& $\overline{V b}(\mathrm{~mm})$ & 0.041 & 0.046 & 0.048 & 0.056 & 0.060 & 0.063 \\
\hline Filo 2 & $\mathrm{P} 1(\mathrm{px})$ & 6.02 & 8.20 & 9.80 & 11.78 & 13.13 & 13.26 \\
& $\mathrm{P} 2(\mathrm{px})$ & 7.22 & 8.99 & 10.41 & 12.67 & 13.89 & 14.59 \\
& $\overline{V b}(\mathrm{px})$ & 6.62 & 8.60 & 10.11 & 12.23 & 13.51 & 13.92 \\
& $\overline{V b}(\mathrm{~mm})$ & 0.024 & 0.031 & 0.036 & 0.044 & 0.049 & 0.050 \\
\hline
\end{tabular}


ANEXOS

\begin{tabular}{llcccccc}
\hline \multirow{2}{*}{ E13 } & & \multicolumn{7}{c}{ Tc $(\mathrm{min})$} \\
\cline { 3 - 7 } & & 5 & 30 & 50 & 60 & 70 & 80 \\
\hline \multirow{2}{*}{ Filo 1 } & $\mathrm{P} 1(\mathrm{px})$ & 31.11 & 34.57 & 35.81 & 38.52 & 40.06 & 42.49 \\
& $\mathrm{P} 2(\mathrm{px})$ & 25.89 & 30.37 & 31.98 & 34.25 & 37.64 & 39.82 \\
& $\overline{V b}(\mathrm{px})$ & 28.50 & 32.47 & 33.89 & 36.38 & 38.85 & 41.16 \\
& $\overline{V b}(\mathrm{~mm})$ & 0.103 & 0.117 & 0.122 & 0.131 & 0.140 & 0.148 \\
\hline \multirow{2}{*}{ Filo 2} & $\mathrm{P} 1(\mathrm{px})$ & 20.79 & 25.32 & 28.44 & 29.43 & 30.05 & 31.97 \\
& $\mathrm{P} 2(\mathrm{px})$ & 16.62 & 22.19 & 24.81 & 25.66 & 26.19 & 27.58 \\
& $\overline{V b}(\mathrm{px})$ & 18.71 & 23.76 & 26.63 & 27.54 & 28.12 & 29.78 \\
& $\overline{V b}(\mathrm{~mm})$ & 0.067 & 0.086 & 0.096 & 0.099 & 0.101 & 0.107 \\
\hline
\end{tabular}

\begin{tabular}{llccccccccc}
\hline \multirow{2}{*}{ E14 } & & \multicolumn{7}{c}{ Tc $(\mathrm{min})$} \\
\cline { 3 - 10 } & & 5 & 7.5 & 10 & 12.5 & 15 & 17.5 & 20 & 30 & 40 \\
\hline Filo 1 & $\mathrm{P} 1(\mathrm{px})$ & 66.84 & 67.09 & 68.04 & 68.76 & 69.14 & 70.07 & 71.65 & 72.16 & 75.00 \\
& $\mathrm{P} 2(\mathrm{px})$ & 50.54 & 52.65 & 53.65 & 54.53 & 60.73 & 62.01 & 63.61 & 64.92 & 68.63 \\
& $\overline{V b}(\mathrm{px})$ & 58.69 & 59.87 & 60.85 & 61.65 & 64.94 & 66.04 & 67.63 & 68.54 & 71.81 \\
& $\overline{V b}(\mathrm{~mm})$ & 0.211 & 0.216 & 0.219 & 0.222 & 0.234 & 0.238 & 0.243 & 0.247 & 0.259 \\
\hline \multirow{2}{*}{ Filo 2 } & $\mathrm{P} 1(\mathrm{px})$ & 57.64 & 59.38 & 61.99 & 63.29 & 65.06 & 65.60 & 66.65 & 67.02 & 69.00 \\
& $\mathrm{P} 2(\mathrm{px})$ & 40.51 & 48.33 & 49.14 & 53.27 & 55.66 & 56.48 & 57.28 & 60.38 & 64.03 \\
& $\overline{V b}(\mathrm{px})$ & 49.07 & 53.86 & 55.56 & 58.28 & 60.36 & 61.04 & 61.96 & 63.70 & 66.52 \\
& $\overline{V b}(\mathrm{~mm})$ & 0.177 & 0.194 & 0.200 & 0.210 & 0.217 & 0.220 & 0.223 & 0.229 & 0.239 \\
\hline
\end{tabular}

\begin{tabular}{|c|c|c|c|c|c|c|c|}
\hline \multirow[t]{2}{*}{ E15 } & & \multicolumn{5}{|c|}{$\mathrm{Tc}(\min )$} & \multirow[b]{2}{*}{80} \\
\hline & & 5 & 30 & 50 & 60 & 70 & \\
\hline \multirow[t]{4}{*}{ Filo 1} & $\mathrm{P} 1$ (px) & 13.27 & 12.88 & 13.91 & 15.36 & 16.26 & 17.23 \\
\hline & $\mathrm{P} 2(\mathrm{px})$ & 11.47 & 11.96 & 12.45 & 13.55 & 14.69 & 14.87 \\
\hline & $\overline{V b}(\mathrm{px})$ & 12.37 & 12.42 & 13.18 & 14.46 & 15.48 & 16.05 \\
\hline & $\overline{V b}(\mathrm{~mm})$ & 0.045 & 0.045 & 0.047 & 0.052 & 0.056 & 0.058 \\
\hline \multirow[t]{4}{*}{ Filo 2} & P1 (px) & 9.76 & 10.06 & 11.87 & 12.51 & 13.60 & 14.33 \\
\hline & P2 (px) & 8.39 & 9.84 & 10.82 & 12.99 & 13.72 & 13.98 \\
\hline & $\overline{V b}(\mathrm{px})$ & 9.07 & 9.95 & 11.34 & 12.75 & 13.66 & 14.16 \\
\hline & $\overline{V b}(\mathrm{~mm})$ & 0.033 & 0.036 & 0.041 & 0.046 & 0.049 & 0.051 \\
\hline
\end{tabular}

\begin{tabular}{llcccccc}
\hline E16 & & \multicolumn{7}{c}{ Tc $(\min )$} \\
\cline { 3 - 7 } & & 5 & 30 & 50 & 60 & 70 & 80 \\
\hline Filo 1 & $\mathrm{P} 1(\mathrm{px})$ & 15.06 & 15.02 & 17.00 & 19.18 & 20.41 & 20.98 \\
& $\mathrm{P} 2(\mathrm{px})$ & 13.68 & 13.82 & 15.80 & 17.90 & 19.16 & 19.63 \\
& $\overline{V b}(\mathrm{px})$ & 14.37 & 14.42 & 16.40 & 18.54 & 19.78 & 20.30 \\
& $\overline{V b}(\mathrm{~mm})$ & 0.052 & 0.052 & 0.059 & 0.067 & 0.071 & 0.073 \\
\hline Filo 2 & $\mathrm{P} 1(\mathrm{px})$ & 7.94 & 10.62 & 11.92 & 14.61 & 15.62 & 18.10 \\
& $\mathrm{P} 2(\mathrm{px})$ & 7.63 & 9.58 & 10.68 & 15.76 & 16.51 & 18.66 \\
& $\overline{V b}(\mathrm{px})$ & 7.78 & 10.10 & 11.30 & 15.19 & 16.07 & 18.38 \\
& $\overline{V b}(\mathrm{~mm})$ & 0.028 & 0.036 & 0.041 & 0.055 & 0.058 & 0.066 \\
\hline
\end{tabular}




\section{ANEXOS AL CAPÍTULO 4}

\section{III.1. EJEMPLO DATOS DE DELAMINADO TIPO II}

Orientación $0^{\circ}-90^{\circ}$

\begin{tabular}{|c|c|c|c|c|c|c|c|c|c|c|c|c|c|c|c|c|c|}
\hline \multirow{2}{*}{$\begin{array}{c}\mathrm{Tc} \\
\mathrm{min}\end{array}$} & \multirow{2}{*}{$\begin{array}{l}\text { E05 } \\
\text { Tipo II }\end{array}$} & & \multicolumn{14}{|c|}{ Mechas } & \multirow{2}{*}{ valor } \\
\hline & & & 1 & 2 & 3 & 4 & 5 & 6 & 7 & 8 & 9 & 10 & 11 & 12 & 13 & 14 & \\
\hline \multirow[t]{3}{*}{30} & Sdi & $\left(\mathrm{mm}^{2}\right)$ & 0.26 & 0.20 & 0.25 & 0.30 & 0.74 & 0.73 & 0.63 & 0.43 & 0.51 & 0.45 & 0.28 & 0.16 & 0.24 & 0.26 & 5.186 \\
\hline & $\mathrm{h}_{\max \mathrm{i}}$ & $(\mathrm{mm})$ & 0.127 & 0.148 & 0.296 & 0.190 & 0.381 & 0.529 & 0.614 & 0.317 & 0.529 & 0.360 & 0.233 & 0.127 & 0.190 & 0.127 & 0.61 \\
\hline & li & $(\mathrm{mm})$ & 1.84 & 2.09 & 1.99 & 2.52 & 2.45 & 2.75 & 2.39 & 1.73 & 2.03 & 1.99 & 1.71 & 1.06 & 1.27 & - & 25.83 \\
\hline \multirow[t]{3}{*}{50} & Sdi & $\left(\mathrm{mm}^{2}\right)$ & 0.31 & 0.42 & 0.46 & 0.23 & 0.36 & 0.49 & 0.11 & 0.34 & 0.35 & 0.44 & 0.38 & 0.59 & 0.49 & 0.32 & 5.297 \\
\hline & $\mathrm{h}_{\max \mathrm{i}}$ & $(\mathrm{mm})$ & 0.21 & 0.19 & 0.19 & 0.28 & 0.30 & 0.23 & 0.19 & 0.34 & 0.28 & 0.30 & 0.25 & 0.40 & 0.32 & 0.28 & 0.40 \\
\hline & li & $(\mathrm{mm})$ & 1.86 & 2.62 & 3.00 & 1.69 & 2.24 & 2.50 & 2.01 & 1.84 & 2.07 & 2.01 & 2.37 & 2.39 & 1.93 & 2.60 & 31.15 \\
\hline \multirow[t]{3}{*}{60} & Sdi & $\left(\mathrm{mm}^{2}\right)$ & 0.47 & 0.51 & 0.52 & 0.48 & 0.68 & 0.37 & 0.41 & 0.37 & 0.19 & 0.30 & 0.27 & - & - & - & 4.565 \\
\hline & $\mathrm{h}_{\max \mathrm{i}}$ & $(\mathrm{mm})$ & 0.507 & 0.232 & 0.296 & 0.211 & 0.211 & 0.253 & 0.211 & 0.169 & 0.169 & 0.190 & 0.084 & - & - & - & 0.51 \\
\hline & li & $(\mathrm{mm})$ & 2.73 & 2.92 & 2.84 & 2.75 & 3.98 & 2.56 & 2.43 & 2.39 & 1.80 & 1.59 & 1.04 & - & - & - & 26.96 \\
\hline \multirow[t]{3}{*}{70} & Sdi & $\left(\mathrm{mm}^{2}\right)$ & 0.61 & 0.46 & 0.61 & 0.88 & 0.93 & 0.50 & 0.85 & 0.84 & 0.77 & 1.01 & 0.58 & 0.12 & 0.26 & - & 8.418 \\
\hline & $\mathrm{h}_{\max \mathrm{i}}$ & $(\mathrm{mm})$ & 0.380 & 0.359 & 0.317 & 0.422 & 0.465 & 0.274 & 0.338 & 0.359 & 0.465 & 0.570 & 0.422 & 0.317 & 0.359 & - & 0.57 \\
\hline & li & $(\mathrm{mm})$ & 1.90 & 1.48 & 1.57 & 2.37 & 2.24 & 2.73 & 3.91 & 3.05 & 3.11 & 3.26 & 2.67 & 0.85 & 1.42 & - & 30.49 \\
\hline \multirow[t]{3}{*}{80} & Sdi & $\left(\mathrm{mm}^{2}\right)$ & 0.48 & 0.65 & 0.46 & 0.42 & 0.25 & 0.30 & 0.16 & 0.24 & 0.13 & 0.14 & 0.39 & 0.26 & 0.26 & - & 4.121 \\
\hline & $\mathrm{h}_{\max \mathrm{i}}$ & $(\mathrm{mm})$ & 0.486 & 0.401 & 0.296 & 0.232 & 0.211 & 0.127 & 0.127 & 0.169 & 0.148 & 0.232 & 0.169 & 0.232 & 0.232 & - & 0.49 \\
\hline & li & $(\mathrm{mm})$ & 0.59 & 3.39 & 2.77 & 2.64 & 2.41 & 2.24 & 1.42 & 1.90 & 1.63 & 1.35 & 2.56 & 1.84 & 1.57 & - & 26.27 \\
\hline
\end{tabular}

\begin{tabular}{|c|c|c|c|c|c|c|c|c|c|c|c|c|c|c|c|c|c|}
\hline \multirow{2}{*}{\multicolumn{2}{|c|}{$\operatorname{mc}_{\min } \frac{\text { E11 }}{\text { Tipo II }}$}} & & \multicolumn{14}{|c|}{ Mechas } & \multirow{2}{*}{ valor } \\
\hline & & & 1 & 2 & 3 & 4 & 5 & 6 & 7 & 8 & 9 & 10 & 11 & 12 & 13 & 14 & \\
\hline \multirow[t]{3}{*}{30} & Sdi & $\left(\mathrm{mm}^{2}\right)$ & 0.80 & 0.37 & 0.79 & 0.64 & 0.70 & 0.55 & 0.65 & 0.72 & 0.86 & 0.66 & 0.46 & 0.74 & 0.39 & 0.37 & 8.685 \\
\hline & $\mathrm{h}_{\max \mathrm{i}}$ & $(\mathrm{mm})$ & 0.475 & 0.289 & 0.537 & 0.475 & 0.516 & 0.330 & 0.434 & 0.578 & 1.487 & 0.558 & 0.372 & 0.413 & 0.413 & 0.330 & 1.49 \\
\hline & li & (mm) & 2.17 & 2.35 & 3.04 & 2.60 & 2.93 & 2.97 & 2.93 & 2.56 & 2.68 & 2.81 & 2.54 & 2.79 & 1.84 & 2.17 & 36.39 \\
\hline \multirow[t]{3}{*}{50} & Sdi & $\left(\mathrm{mm}^{2}\right)$ & 0.87 & 0.81 & 1.69 & 1.96 & 1.70 & 1.43 & 1.60 & 1.89 & 2.78 & 2.34 & 2.36 & 1.34 & 1.31 & 1.29 & 23.354 \\
\hline & $\mathrm{h}_{\max \mathrm{i}}$ & $(\mathrm{mm})$ & 0.73 & 0.67 & 1.22 & 1.18 & 1.36 & 1.32 & 1.34 & 1.42 & 1.60 & 1.85 & 2.05 & 2.17 & 1.95 & 2.35 & 2.35 \\
\hline & li & (mm) & 1.65 & 2.81 & 2.60 & 2.71 & 2.48 & 2.48 & 2.40 & 2.44 & 2.73 & 2.60 & 2.54 & 2.25 & 3.30 & 2.07 & 34.45 \\
\hline \multirow[t]{3}{*}{60} & Sdi & $\left(\mathrm{mm}^{2}\right)$ & 0.41 & 2.16 & 2.44 & 2.85 & 4.73 & 2.90 & 4.04 & 3.91 & 2.34 & 3.72 & 3.25 & 3.53 & 2.73 & 3.38 & 42.396 \\
\hline & $\mathrm{h}_{\max \mathrm{i}}$ & $(\mathrm{mm})$ & 0.888 & 1.364 & 1.880 & 2.045 & 1.839 & 1.839 & 1.818 & 1.963 & 1.901 & 1.963 & 1.921 & 2.025 & 1.983 & 1.983 & 2.05 \\
\hline & li & $(\mathrm{mm})$ & 1.78 & 2.48 & 2.85 & 2.64 & 3.28 & 2.79 & 2.68 & 2.71 & 2.42 & 2.58 & 2.52 & 2.62 & 2.21 & 2.48 & 36.05 \\
\hline \multirow[t]{3}{*}{70} & Sdi & $\left(\mathrm{mm}^{2}\right)$ & 0.76 & 1.64 & 1.54 & 0.28 & 1.76 & 2.42 & 2.64 & 2.52 & 0.58 & 2.05 & 2.09 & 2.10 & 1.61 & - & 21.985 \\
\hline & $\mathrm{h}_{\max \mathrm{i}}$ & $(\mathrm{mm})$ & 0.540 & 2.138 & 2.034 & 0.311 & 1.785 & 1.993 & 2.325 & 2.304 & 0.643 & 2.055 & 2.200 & 2.013 & 2.076 & - & 2.32 \\
\hline & li & $(\mathrm{mm})$ & 2.42 & 2.52 & 2.11 & 1.34 & 2.52 & 2.46 & 2.52 & 2.21 & 1.94 & 2.23 & 2.48 & 2.13 & 2.58 & - & 29.60 \\
\hline \multirow[t]{3}{*}{80} & Sdi & $\left(\mathrm{mm}^{2}\right)$ & 0.33 & 0.54 & 0.58 & 1.28 & 2.37 & 3.10 & 1.84 & 1.93 & 1.42 & 1.26 & 0.76 & 1.43 & 3.17 & 2.06 & 22.078 \\
\hline & $\mathrm{h}_{\max \mathrm{i}}$ & $(\mathrm{mm})$ & 0.225 & 0.306 & 0.388 & 2.104 & 2.002 & 2.083 & 1.593 & 2.022 & 1.185 & 1.430 & 0.327 & 1.532 & 2.022 & 1.675 & 2.10 \\
\hline & li & $(\mathrm{mm})$ & 1.90 & 2.60 & 2.81 & 2.54 & 2.73 & 2.60 & 2.95 & 3.16 & 3.18 & 2.75 & 3.30 & 2.64 & 2.75 & 2.50 & 37.99 \\
\hline
\end{tabular}

\begin{tabular}{|c|c|c|c|c|c|c|c|c|c|c|c|c|c|c|c|c|c|}
\hline \multirow{2}{*}{\multicolumn{2}{|c|}{$\begin{array}{cc}\text { Tc } \\
\text { min }\end{array} \frac{\text { E12 }}{\text { Tipo II }}$}} & & \multicolumn{14}{|c|}{ Mechas } & \multirow{2}{*}{ valor } \\
\hline & & & 1 & 2 & 3 & 4 & 5 & 6 & 7 & 8 & 9 & 10 & 11 & 12 & 13 & 14 & \\
\hline \multirow[t]{3}{*}{30} & Sdi & $\left(\mathrm{mm}^{2}\right)$ & 1.12 & 0.86 & 1.06 & 1.05 & 1.29 & 0.90 & 1.31 & 1.22 & 0.93 & 0.54 & 0.57 & 0.80 & 0.45 & 0.22 & 12.33 \\
\hline & $\mathrm{h}_{\max \mathrm{i}}$ & $(\mathrm{mm})$ & 0.70 & 0.82 & 0.46 & 0.95 & 0.55 & 0.46 & 0.99 & 0.82 & 0.76 & 0.40 & 0.53 & 0.53 & 0.27 & 0.34 & 0.99 \\
\hline & li & (mm) & 2.00 & 2.13 & 3.48 & 2.57 & 3.16 & 2.93 & 2.82 & 2.51 & 2.53 & 1.96 & 1.71 & 2.17 & 2.80 & 0.72 & 33.47 \\
\hline \multirow[t]{3}{*}{50} & Sdi & $\left(\mathrm{mm}^{2}\right)$ & 0.41 & 0.38 & 0.32 & 0.31 & 0.40 & 0.42 & 0.45 & 0.32 & 0.27 & 0.31 & 0.16 & 0.48 & 0.35 & 0.78 & 5.35 \\
\hline & $\mathrm{h}_{\max \mathrm{i}}$ & $(\mathrm{mm})$ & 0.27 & 0.23 & 0.23 & 0.27 & 0.23 & 0.38 & 0.44 & 0.36 & 0.25 & 0.32 & 0.44 & 0.53 & 0.32 & 0.72 & 0.71 \\
\hline & $\mathrm{li}$ & (mm) & 2.44 & 2.78 & 2.75 & 2.59 & 2.96 & 2.38 & 2.57 & 2.00 & 2.04 & 2.14 & 1.14 & 2.61 & 2.10 & 1.89 & 32.38 \\
\hline \multirow[t]{3}{*}{60} & Sdi & $\left(\mathrm{mm}^{2}\right)$ & 0.41 & 0.38 & 0.32 & 0.31 & 0.40 & 0.42 & 0.45 & 0.32 & 0.27 & 0.31 & 0.16 & 0.48 & 0.35 & 0.78 & 5.35 \\
\hline & $\mathrm{h}_{\max \mathrm{i}}$ & $(\mathrm{mm})$ & 0.29 & 0.23 & 0.22 & 0.26 & 0.20 & 0.38 & 0.44 & 0.35 & 0.25 & 0.30 & 0.43 & 0.53 & 0.29 & 0.71 & 0.71 \\
\hline & li & (mm) & 2.42 & 2.75 & 2.73 & 2.57 & 2.94 & 2.35 & 2.54 & 1.98 & 2.02 & 2.12 & 1.11 & 2.59 & 2.08 & 1.87 & 32.08 \\
\hline \multirow[t]{3}{*}{70} & Sdi & $\left(\mathrm{mm}^{2}\right)$ & 0.42 & 0.27 & 0.20 & 0.64 & 0.32 & 0.35 & 0.36 & 1.01 & 0.39 & 0.97 & - & 0.39 & - & 0.07 & 5.39 \\
\hline & $\mathrm{h}_{\max \mathrm{i}}$ & $(\mathrm{mm})$ & 0.44 & 0.29 & 0.36 & 0.78 & 0.27 & 0.25 & 0.19 & 0.91 & 0.67 & 0.99 & - & 0.38 & - & 0.21 & 0.99 \\
\hline & $\mathrm{li}$ & $(\mathrm{mm})$ & 1.45 & 2.09 & 1.12 & 2.21 & 1.96 & 3.24 & 2.91 & 2.74 & 2.51 & 2.02 & - & 1.90 & - & 5.31 & 31.07 \\
\hline \multirow[t]{3}{*}{80} & Sdi & $\left(\mathrm{mm}^{2}\right)$ & 0.87 & 0.90 & 0.88 & 0.90 & 0.72 & 0.60 & 0.64 & 0.53 & 0.61 & 0.66 & 0.36 & 0.37 & 0.48 & 0.48 & 9.03 \\
\hline & $\mathrm{h}_{\max \mathrm{i}}$ & $(\mathrm{mm})$ & 0.53 & 0.53 & 0.63 & 0.53 & 0.63 & 0.40 & 0.36 & 0.40 & 0.34 & 0.49 & 0.40 & 0.30 & 0.34 & 0.32 & 0.63 \\
\hline & li & $(\mathrm{mm})$ & 2.60 & 2.20 & 1.99 & 2.18 & 2.09 & 2.43 & 2.03 & 1.73 & 2.51 & 2.77 & 2.03 & 2.15 & 2.32 & 2.72 & 31.74 \\
\hline
\end{tabular}


ANEXOS

\begin{tabular}{|c|c|c|c|c|c|c|c|c|c|c|c|c|c|c|c|c|c|}
\hline \multirow{2}{*}{$\begin{array}{l}\text { Tc } \\
\text { min }\end{array}$} & \multirow{2}{*}{$\frac{\text { E14 }}{\text { Tipo II }}$} & & \multicolumn{14}{|c|}{ Mechas } & \multirow{2}{*}{ valor } \\
\hline & & & 1 & 2 & 3 & 4 & 5 & 6 & 7 & 8 & 9 & 10 & 11 & 12 & 13 & 14 & \\
\hline & Sdi & $\left(\mathrm{mm}^{2}\right)$ & 0.44 & 0.49 & 0.63 & 0.67 & 2.02 & 1.35 & 2.98 & 0.79 & 2.02 & 3.03 & 2.41 & 3.16 & 1.91 & 1.50 & 23.413 \\
\hline \multirow{2}{*}{ 1. 1} & $\mathrm{~h}_{\max \mathrm{i}}$ & $(\mathrm{mm})$ & 0.45 & 0.51 & 1.69 & 1.62 & 2.22 & 1.60 & 2.07 & 1.13 & 1.60 & 1.71 & 1.32 & 1.54 & 1.67 & 1.60 & 2.22 \\
\hline & li & $(\mathrm{mm})$ & - & 2.01 & 2.09 & 2.75 & 1.58 & 2.24 & 2.80 & 2.80 & 2.63 & 3.35 & 3.63 & 3.35 & 3.65 & 4.46 & 37.34 \\
\hline \multirow[t]{3}{*}{5} & Sdi & $\left(\mathrm{mm}^{2}\right)$ & 1.66 & 4.53 & 2.80 & 2.42 & 3.89 & 3.52 & 2.36 & 3.18 & 5.69 & 4.07 & 4.17 & 4.37 & 4.67 & 4.08 & 51.415 \\
\hline & $\mathrm{h}_{\max \mathrm{i}}$ & $(\mathrm{mm})$ & 1.54 & 1.99 & 2.05 & 1.74 & 2.01 & 2.05 & 2.07 & 2.03 & 2.01 & 2.01 & 2.09 & 2.11 & 1.95 & 1.93 & 2.11 \\
\hline & li & $(\mathrm{mm})$ & 2.03 & 2.88 & 2.26 & 3.05 & 3.07 & 2.67 & 2.99 & 2.75 & 4.08 & 3.03 & 2.60 & 2.78 & 2.80 & 2.65 & 38.03 \\
\hline \multirow[t]{3}{*}{10} & Sdi & $\left(\mathrm{mm}^{2}\right)$ & 4.49 & 4.91 & 3.77 & 3.72 & 4.21 & 4.12 & 3.75 & 3.50 & 2.94 & 2.54 & 2.53 & 1.29 & 1.28 & - & 43.036 \\
\hline & $\mathrm{h}_{\max \mathrm{i}}$ & $(\mathrm{mm})$ & 2.58 & 2.03 & 1.66 & 1.54 & 1.54 & 1.58 & 1.50 & 1.78 & 1.39 & 1.44 & 1.15 & 1.00 & 1.13 & - & 2.58 \\
\hline & li & $(\mathrm{mm})$ & 2.35 & 2.82 & 3.03 & 3.07 & 3.07 & 3.22 & 3.07 & 2.84 & 3.03 & 2.84 & 2.97 & 3.03 & 1.77 & - & 35.66 \\
\hline \multirow[t]{3}{*}{20} & Sdi & $\left(\mathrm{mm}^{2}\right)$ & 2.22 & 2.82 & 2.90 & 3.72 & 2.92 & 3.17 & 3.72 & 2.70 & 3.62 & 3.23 & 2.76 & 1.77 & 1.55 & - & 37.096 \\
\hline & $\mathrm{h}_{\max \mathrm{i}}$ & $(\mathrm{mm})$ & 1.31 & 1.53 & 1.55 & 1.55 & 1.57 & 1.53 & 1.60 & 1.57 & 1.39 & 1.51 & 1.33 & 1.04 & 0.94 & - & $\begin{array}{l}1.60 \\
\end{array}$ \\
\hline & li & $(\mathrm{mm})$ & 2.39 & 2.60 & 2.93 & 3.33 & 2.50 & 2.43 & 2.60 & 2.14 & 3.07 & 2.69 & 2.52 & 2.86 & 2.35 & - & 32.97 \\
\hline \multirow[t]{3}{*}{30} & Sdi & $\left(\mathrm{mm}^{2}\right)$ & 2.11 & 2.94 & 0.99 & 2.30 & 3.12 & 4.11 & 3.55 & 4.42 & 4.00 & 3.60 & 3.42 & 3.52 & 3.13 & - & 41.210 \\
\hline & $\mathrm{h}_{\max \mathrm{i}}$ & $(\mathrm{mm})$ & 2.39 & 2.47 & 1.55 & 2.43 & 2.24 & 2.18 & 2.26 & 1.98 & 1.80 & 2.04 & 1.51 & 1.47 & 1.49 & - & 2.47 \\
\hline & li & $(\mathrm{mm})$ & 2.33 & 2.39 & 2.20 & 2.54 & 2.41 & 2.41 & 2.56 & 2.67 & 2.69 & 2.33 & 2.95 & 2.46 & 2.43 & - & 30.92 \\
\hline \multirow[t]{3}{*}{37.5} & Sdi & $\left(\mathrm{mm}^{2}\right)$ & 3.00 & 5.55 & 3.34 & 3.31 & 4.59 & 2.69 & 2.45 & 3.06 & 2.33 & 2.15 & 1.34 & 2.69 & 2.22 & - & 38.733 \\
\hline & $\mathrm{h}_{\max \mathrm{i}}$ & $(\mathrm{mm})$ & 2.506 & 2.444 & 2.280 & 1.910 & 2.054 & 1.807 & 1.417 & 1.520 & 1.787 & 1.417 & 1.253 & 1.417 & 1.315 & - & 2.51 \\
\hline & $\mathrm{li}$ & $(\mathrm{mm})$ & 2.03 & 2.75 & 2.67 & 2.26 & 2.80 & 2.31 & 1.88 & 2.90 & 2.07 & 2.33 & 2.28 & 2.73 & 2.11 & - & 29.95 \\
\hline \multirow[t]{3}{*}{40} & Sdi & $\left(\mathrm{mm}^{2}\right)$ & 5.01 & 4.69 & 5.01 & 4.92 & 4.81 & 4.76 & 4.76 & 3.95 & 5.30 & 5.53 & 5.03 & 4.27 & 4.87 & 4.37 & 67.291 \\
\hline & $\mathrm{h}_{\max \mathrm{i}}$ & $(\mathrm{mm})$ & 2.519 & 2.683 & 2.663 & 2.642 & 2.704 & 2.949 & 2.663 & 2.663 & 2.704 & 2.826 & 2.826 & 2.867 & 3.011 & 3.011 & 3.01 \\
\hline & li & $(\mathrm{mm})$ & 1.86 & 2.54 & 3.07 & 2.54 & 2.58 & 3.61 & 2.93 & 2.50 & 2.73 & 2.80 & 2.46 & 2.80 & 2.67 & 2.33 & 35.88 \\
\hline
\end{tabular}

